Marcus Poggenpohl

Verbundanalyse im Einzelhandel

auf der Grundlage

von Kundenkarten-

informationen 


\section{Marcus Poggenpohl}

\section{Verbundanalyse im Einzelhandel auf der Grundlage von Kundenkarteninformationen}

Der Handel steckt bei planerischen Entscheidungen häufig in einem Informationsdilemma. Unternehmenseigene Kundenkarten als neues Marketinginstrument können diesen Mangel beheben. Auch die Verbundforschung, die Beziehungen zwischen verschiedenen Sortimentsbereichen untersucht, kann dadurch weiterentwickelt werden. Bei über 4500 Kunden im Laufe eines Jahres erhobene Kauf- und Personendaten ermöglichen erstmals eine zeitraumorientierte Verbundanalyse. Grundlage dafür ist eine systematische Erfassung aller Einflußgrößen und eine exakte Operationalisierung. Aufbauend auf den ermittelten Verbundbeziehungen können Zielgruppen mit hoher Kaufverhaltensrelevanz anhand von Verbundkaufmustern gebildet werden. Für die Handelspraxis werden darüber hinaus Implikationen und Anwendungsbeispiele aufgezeigt.

Marcus Poggenpohl wurde 1962 in Hagen/Westfalen geboren. Er studierte Betriebswirtschaft an der RWTH Aachen. Nach dem Abschluß arbeitete er zunächst im Bereich Marketing/EDV im Investitionsgüterbereich. Während der Promotion arbeitete er als wissenschaftlicher Assistent am Institut für Marketing der WWU Münster und als Projektleiter im Marketing eines Warenhauskonzerns. 
Verbundanalyse im Einzelhandel auf der Grundlage von Kundenkarteninformationen

Eine empirische Untersuchung von Verbundbeziehungen

zwischen Abteilungen 


\section{Schriften zu Marketing und Manasement}

Herausgegebenvon Prof.Dr. Dr.h.c. Heribert Meffert

Band 22

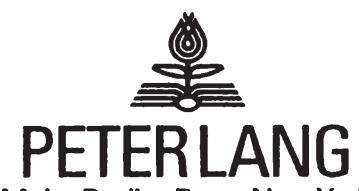

Frankfurtam Main · Berlin · Bern. NewYork · Paris · Wien

Marcus Poggenpohl - 978-3-631-75099-5

Downloaded from PubFactory at 01/11/2019 08:22:38AM

via free access 


\title{
Marcus Poggenpohl
}

\author{
Verbundanalyse \\ imEinzelhandel \\ aufderGrundlagevon \\ Kundenkarteninformationen
}

Eine empirische Untersuchung von

Verbundbeziehungen zwischen Abteilungen

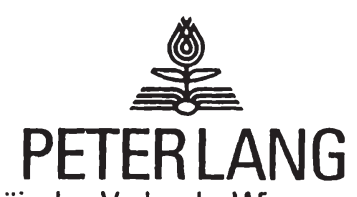

EuropäischerVerlag derWissenschaften

Marcus Poggenpohl - 978-3-631-75099-5

Downloaded from PubFactory at 01/11/2019 08:22:38AM 
Die Deutsche Bibliothek - CIP-Einheitsaufnahme

Poggenpohl, Marcus:

Verbundanalyse im Einzelhandel auf der Grundlage von Kundenkarteninformationen : eine empirische Untersuchung von Verbundbeziehungen zwischen Abteilungen / Marcus Poggenpohl. - Frankfurt am Main ; Berlin ; Bern ; New York ; Paris ; Wien : Lang, 1994

(Schriften zu Marketing und Management ; Bd. 22)

Zugl.: Münster (Westfalen), Univ., Diss., 1993

ISBN 3-631-46805-9

NE: GT

Open Access: The online version of this publication is published on www.peterlang.com and www.econstor.eu under the international Creative Commons License CC-BY 4.0. Learn more on how you can use and share this work: http://creativecommons. org/licenses/by/4.0.

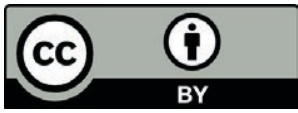

This book is available Open Access thanks to the kind support of ZBW - Leibniz-Informationszentrum Wirtschaft.

\author{
D 6 \\ ISSN 0176-2729 \\ ISBN 3-631-46805-9 \\ ISBN 978-3-631-75099-5 (eBook) \\ (C) Peter Lang GmbH \\ Europäischer Verlag der Wissenschaften \\ Frankfurt am Main 1994 \\ Alle Rechte vorbehalten.
}

Das Werk einschließlich aller seiner Teile ist urheberrechtlich geschützt. Jede Verwertung außerhalb der engen Grenzen des

Urheberrechtsgesetzes ist ohne Zustimmung des Verlages unzulässig und strafbar. Das gilt insbesondere für

Vervielfältigungen, Übersetzungen, Mikroverfilmungen und die Einspeicherung und Verarbeitung in elektronischen Systemen. 


\section{Für meine Eltern}

\section{Ohne sie wäre das alles nicht möglich gewesen}


Marcus Poggenpohl - 978-3-631-75099-5

Downloaded from PubFactory at 01/11/2019 08:22:38AM

via free access 
Vorwort des Herausgebers

Das Sortiment steht als zentraler Erfolgsfaktor im Mittelpunkt planerischer Konzepte der Handelspraxis. Bei strategischen und taktischen Entscheidungen wird die optimale Auswahl und Zusammenstellung von Sortimentsbestandteilen angestrebt. Die Handelsunternehmen orientieren sich dabei überwiegend an heuristisch ermittelten Erfahrungsgrößen.

Die wissenschaftliche Forschung hat der Sortimentsplanung immer schon ein besonderes Augenmerk gewidmet. Die zahlreichen Veröffentlichungen zur Sortimentsoptimierung legen davon ein beredtes Zeugnis ab. Dazu gehört auch die Erfassung der $\mathrm{Zu}$ sammenhänge zwischen Sortimentsbestandteilen aus Kundensicht im Rahmen der Verbundforschung.

Schon aus früheren Arbeiten zu diesem Problemkreis ließen sich für die Handelspraxis wertvolle Hinweise zur Sortimentsgestaltung ableiten. Die Ansätze waren jedoch mit einigen Schwächen behaftet. Dem enormen Informationsbedarf stand ein sehr hoher Informationsbeschaffungsaufwand gegenüber, so daß viele Analysen nur mit kleinen stichproben durchgeführt wurden. Zudem waren die Voraussetzungen für eine DV-technische Verarbeitung meist nicht gegeben. Darüber hinaus erfolgte die Erfassung der Verbundkäufe eines Konsumenten und damit von Verbundbeziehungen zwischen Sortimentsbestandteilen nur zeitpunktbezogen. Es war nicht möglich einen speziellen Kunden bei erneuten Käufen zu identifizieren.

Mit der Einführung von Scanningkassen und der Verbesserung der EDV-Systeme konnte das Dilemma des Informationsmangels z.T. überwunden werden. Offen blieb jedoch die Zuordnung von Kauf- und Personendaten zu einzelnen Kaufakten.

Unternehmenseigenene Kundenkarten zeigen hier neue Möglichkeiten auf. Neben finanzwirtschaftlichen und marketingbezoge- 
nen Zielsetzungen weisen diese Karten große Potentiale bzgl. der Informationsbeschaffung, insbesondere auch für die verbesserte Verbundmessung im Handel auf. Der benötigte Informationsumfang fällt durch die Speicherung von Kaufdaten automatisch an und die Speicherung der Personendaten ermöglicht zudem die Kundenidentifikation im Zeitablauf.

Die vorliegende Untersuchung sucht die verbesserten technischen Möglichkeiten zu nutzen, um die Verbundforschung im Handel weiterzuentwickeln. Dabei steht die Entwicklung und Messung einer zeitraumbezogenen Verbundgröße im Mittelpunkt. Auf der Grundlage eines umfassenden Systems von Einflußparametern auf Verbundbeziehungen werden Hypothesen im Rahmen einer empirischen Analyse der Käufe eines Jahres von mehr als 4.500 Kunden eines Handelsunternehmens getestet.

Ausgehend von den Zielsetzungen und Möglichkeiten der Kundenkarten von Handelsunternehmen stellt der Verfasser die quantitativen und qualitativen Aspekte der Informationsgewinnung in den Vordergrund. Er zeigt die relevanten Dimensionen von Kundenkarten auf und analysiert die Möglichkeiten dieses neuen Instrumentes für das Marketing.

Im theoretischen Hauptteil der Arbeit werden zunächst zentrale Begriffe der Verbundforschung abgegrenzt und bestehende Konzepte zur Erfassung des Sortimentsverbundes analytisch aufgearbeitet. Bei der Entwicklung eines Systems von Einflußgrößen auf die Entstehung und Ausgestaltung von Verbundbeziehungen werden sowohl Aspekte der Angebots-als auch der Nachfrageseite in die Betrachtung einbezogen. Die Einflüsse von Warenpräsentation, Preissetzung, Plazierung etc. finden dabei ebenso Berücksichtigung wie das komplexe Feld des Konsumentenverhaltens. Große Sorgfalt legt der Verfasser auf die Operationalisierung des Verbundbegriffes. Desweiteren zeigt er die Besonderheiten auf, die die Verwendung von Kundenkarteninformationen bei der Verbundmessung mit sich bringt. 
Die empirische Analyse befaßt sich mit der Ermittlung und Interpretation von zeitpunkt- und zeitraumbezogenen Verbundbeziehungen zwischen Abteilungen. Die erfaßten Beziehungsstrukturen werden auf ihre Plausibilität geprüft und Erklärungsan. sätze für Abweichungen von plausiblen Strukturen entwickelt. Darüber hinaus gelingt es Zielgruppen des Handels auf Basis von Verbundinformationen abzuleiten und die Kunden des untersuchten Handelsunternehmens anhand ihrer spezifischen Verbundkaufmuster in Gruppen einzuteilen.

Im abschließenden Teil werden aufschlußreiche Implikationen für die Handelspraxis abgeleitet. Dabei steht die Darstellung von Anwendungsbereichen und -beispielen im Vordergrund.

Insgesamt gelingt es dem Verfasser die Tradition der verbundanalytischen Untersuchungen fortzuführen und weiterzuentwickeln. Dabei werden klassische Konzepte an die verbesserten technischen Möglichkeiten angepaßt und neue Dimensionen für die Sortimentspolitik im Handel aufgezeigt.

Münster, im Juni 1993

H. Meffert 
Marcus Poggenpohl - 978-3-631-75099-5

Downloaded from PubFactory at 01/11/2019 08:22:38AM

via free access 
Handelsunternehmen sind bei ihren planerischen Entscheidungen zunehmend auf qualifizierte Informationen über die Bedürfnisse ihrer Kunden angewiesen. Dies gilt insbesondere für die optimale Ausgestaltung ihrer Sortimente. Befragungen erweisen sich in diesem Zusammenhang häufig als unzureichende Informationsquelle. Zudem können auf diese Weise gewonnene Kenntnisse kaum das komplexe system der verschiedenen Sortimentsbereiche und der zwischen ihnen bestehenden Wechselwirkungen erfassen.

In diesem Bereich bemüht sich die Verbundforschung seit den 70 er Jahren der Handelspraxis wertvolle Hinweise zu geben. Dabei wurden bisher jedoch zumeist unzureichende Daten zugrunde gelegt, da die Informationsbeschaffung zumeist sehr aufwendig ist. Insbesondere der notwendige Datenumfang und die fehlende Verknüpfung von Kauf-und Personendaten ist in diesem Zusammenhang problematisch.

Die zunehmende Verbreitung unternehmenseigenener Kundenkarten bietet hier neue Möglichkeiten. Das ursprünglich vor allem zur Kundenbindung entwickelte Marketinginstrument hat auch große Potentiale bei der Informationsbeschaffung. Die Kaufdaten fallen bei jeder Zahlung mit der Kundenkarte automatisch an und die speicherung der personendaten ermöglicht zudem die Kundenidentifikation im Zeitablauf .

Die vorliegende Arbeit will vor diesem Hintergrund die ver. besserte Informationsbasis für eine Weiterentwicklung der Verbundforschung im Handel nutzen. Insbesondere die zeitraumbezogene Erfassung von Verbundbeziehungen soll dabei neue Erkenntnisse ermöglichen. Darüber hinaus werden die Ergebnisse der Verbundanalysen zur zielgruppenbildung genutzt.

Bei der Fertigstellung dieser Arbeit unterstützten mich ver schiedene Personen. Meinem akademischen Lehrer Prof. Dr. Dr. 
h.c. Heribert Meffert gebührt großer Dank für die Anregung der Themenstellung, seine ständige Diskussionsbereitschaft und Geduld. Prof. Dr. Diether Ahlert sei für die Übernahme des Zweitgutachtens gedankt.

Dank gilt weiterhin den Kolleginnen und Kollegen am Institut für Marketing für ihre Unterstützung. Besondere Erwähnung gebührt dabei einigen besonders engagierten Persönlichkeiten. Prof. Dr. Martin Benkenstein und seiner Familie für die Unterstützung in jeder Phase der Arbeit. Herr Dipl.-Kfm. Kai Bauche teilte mit mir sein DV-technisches wissen bei der empirischen Arbeit und war stets diskussionsbereit. Frau Dr. Kerstin Christiane Monhemius diskutierte mit mir kritisch die Käuferverhaltensaspekte meiner Arbeit. Nicht zuletzt danke ich Frau Marga Bürger für die andauernde Unterstützung nicht nur bei der Manuskripterstellung und viele wertvolle Tips.

Mein gesamter Werdegang von der Schule bis zur promotion wurde durch die andauernde Unterstützung meiner Eltern intensiv gefördert. Ihnen gilt mein besonderer Dank

Münster, im Juni 1993

Marcus Poggenpohl 


\section{INHALTSVERZEICHNIS}

A. KUNDENKARTENINFORMATIONEN ALS GRUNDLAGE DER VERBUNDANALYSE IM EINZELHANDEL ................... 1

1. Die Bedeutung von Verbundanalysen für das Handelsmarketing ............................ 1

2. Die Entwicklung des Kredit- und Kundenkartenmarktes in der Bundesrepublik unter besonderer Berücksichtigung des Einzelhandels .................... 6

3. Ziele, Ausgestaltungsformen und Einsatzbereiche von Kundenkarten .......................... 15

4. Informationspotentiale von Kundenkarten für den Einzelhandel ......................... 24

5. Zielsetzung und Gang der Untersuchung ............ 29

B. THEORETISCHE GRUNDLAGEN DER VERBUNDMESSUNG IM HANDEL . . 32

1. Ansätze zur verbundmessung im Handel ............ 32

1.1 Das System unterschiedlicher verbundarten ........ 32

1.2 Forschungsansätze zur Verbundanalyse .......... 37

1.21 Klassische Ansätze zur Verbundmessung ...... 37

1.22 Empirische Konzepte zur Erfassung des Verbundes im Einzelhandel ..............4 4

1.221 Empirische Untersuchungen im Überblick ................... 41

1.222 Probabilistische Meßmodelle als Grundlage der Verbundanalyse ............44

1.223 Ermittlung eines Bekanntheitsverbundes zwischen Abteilungen durch Faktorenbildung ................... 48

1.23 Die Transformation von Kaufverbundhäufigkeitsmatrizen mittels Regression, Korrelation und Anwendung von Ähnlichkeitsmaßen ..... 50

1.24 Die Verwendung multivariater Analysemethoden bei der Verbundanlyse ............... 54

1.241 Faktorenanalyse ............... 54

1.242 Multidimensionale Skalierung ........ 56

1.243 Clusteranalyse ................ 58 
2. Die Erfassung von Kauf- und Nachfrageverbund im Handel .............................. 60

2.1 Einflußgrößen und Bestimmungsfaktoren des Kaufund Nachfrageverbundes ..................6 60

2.11 Angebots - bzw. unternehmensbezogene Einflußgrößen des Kauf- und Nachfrageverbundes ......664

2.12 Konsumentenbezogene Einflußgrößen des Kaufund Nachfrageverbundes ...............668

2.13 Wettbewerbsbezogene Einflußgrößen des Kauf und Nachfrageverbundes .............. 76

2.14 Indirekte Einflußgrößen des Kauf- und Nachfrageverbundes ................. 78

2.2 Operationalisierungsansätze bei der Verbundmes sung .............................. 80

2.21 Typen von Verbundwirkungen ............ 80

2.22 Symmetrische und asymmetrische Verbundbeziehungen ...................... 85

2.23 Das Aggregationsniveau als Bestimmungsfaktor der Verbundbeziehung ................ 89

2.24 Verbundmessung anhand von Absatz- oder Umsatzgrößen ..................... 91

3. Besonderheiten der Verbundmessung mit Kundenkarten .....93

3.1 Struktur und Inhalte von Kundenkarteninformatio-

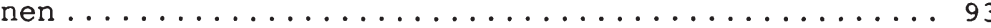

3.2 Zielgruppenspezifische Analysemöglichkeiten auf grund der Zurechenbarkeit sozioökonomischer In-

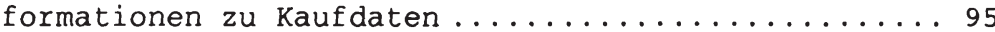

3.3 Mehrfachmessung von Kaufverbunden gleichbleiben der Kundengruppen auf Basis der Identifizierbarkeit von Kunden zu verschiedenen Kaufzeitpunkten ... 97

3.4 Die Erfassung von Kauf- und Nachfrageverbund auf der Basis von Kundenkarteninformationen .......... 99

3.41 Der Kaufverbund als zeitpunktbezogener Ansatz der Verbundmessung ................ 99

3.42 Der Nachfrageverbund als zeitraumbezogener Ansatz der Verbundmessung .............. 105

3.5 Zielgruppenbildung anhand von Verbundinformatio

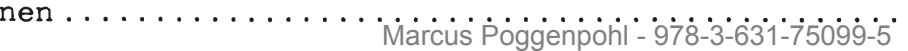


C. EMPIRISCHE ANALYSEN DES KAUF- UND NACHFRAGEVERBUNDES

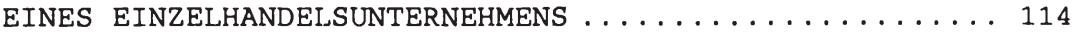

1. Design der Untersuchung ................... 114

1.1 Kennzeichnung der Stichprobe und Struktur der Daten ............................... 114

1.2 Kontextdimensionen der analysierten Geschäfts-

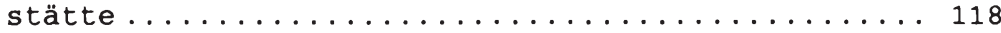

1.21 Geschäftsstättenbezogene Aspekte ......... 118

1.22 Wettbewerbsbezogene Aspekte ........... 122

1.3 Operationalisierung des Verbundbegriffes für die empirische Analyse .................... 124

1.4 Festlegung der Untersuchungsgruppen nach demographischen und ökonomischen Kriterien .......... 126

2. Der Kaufverbund als zeitpunktbezogener Ansatz der Verbundmessung ......................... 129

2.1 Sortimentsgruppenbildung und verbundintensität in der Gesamtstichprobe ................ 130

2.2 Überprüfung der Einflüsse der Kontextdimensionen der Geschäftsstätte auf die Sortimentsgruppenbil-

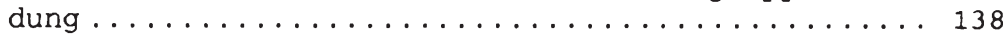

3. Der Nachfrageverbund als zeitraumbezogener Ansatz der Verbundmessung ....................... 141

3.1 Verbundgruppenbildung in der Gesamtstichprobe .... 142

3.2 Verbundintensität und Sortimentsgruppenbildung in ausgewählten sozioökonomischen Zielgruppen ....... 152

3.21 Analyse der abteilungsbezogenen Intensitätswerte........................ 152

3.22 Faktorenbildung in ausgewählten Altersklas.

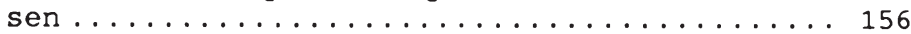

3.23 Faktorenbildung in ausgewählten Einkommens-

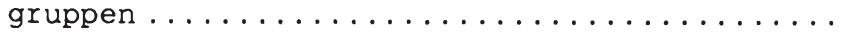


4. Zielgruppenbildung anhand von Verbundbeziehungen im Einzelhandel ........................ 166

4.1 Der Nachfrageverbund als Ausgangspunkt der Zielgruppenbildung .................... 166

4.2 Kundensegmentierung auf der Basis von Nachfrageverbundbeziehungen ................. 168

4.3 Die Gruppe der inaktiven Kartenbesitzer ......... 175

5. Implikationen des Kauf- und Nachfrageverbundes für das Management im Einzelhandel .............. 177

5.1 Kritische würdigung der Leistungsfähigkeit der Verbundanalyseverfahren ................. 177

5.2 Anwendungsbereiche des Kaufverbundes im Rahmen des Handelsmarketing .................. 185

5.21 Die Zusammensetzung von Geschäftseinheiten ... 185

5.22 Plazierungsentscheidungen auf der Basis von Kaufverbundbeziehungen ............... 189

5.23 Auswirkungen der Eliminierung von Abteilungen ...................... 191

5.24 Kaufverbundbeziehungen als Grundlage zur Ermittlung von Kundenwegen in der Geschäfts stätte .................... 196

5.3 Anwendungsbereiche des Nachfrageverbundes im Rahmen des Handelsmarketing ................. 200

5.31 Implikationen für die Kommunikationspolitik im Rahmen eines Data-Base-Marketing ........200

5.32 Implikationen für die Sortimentspolitik .... 204

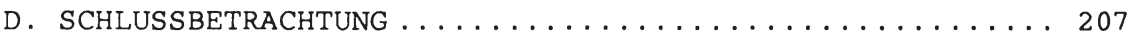

1. Zusammenfassende würdigung der Untersuchungsergebnisse ........................... 207

2. Ansatzpunkte für die weitere Forschung .......... 211

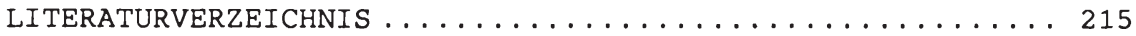




\section{ABBILDUNGSVERZEICHNIS}

Abb. 1: Kreditkartenumsätze in der BRD 1986-1989 (ohne Warenhausumsätze) ................... 7

Abb. 2: Anteile von Zahlungsarten in US-Handelsunterneh-

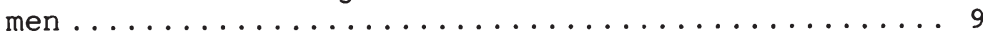

Abb. 3: Strukturierung von Kartensystemen in Deutschland ........................... 10

Abb. 4: Positionierung von Kundenkartentypen in einer Nutzenmatrix ..................... 12

Abb. 5: Ziele der Ausgabe von Kundenkarten ........... 17

Abb. 6: Entwicklungsstufen der Kundenbindung .......... 19

Abb. 7: Ausstattungsmerkmale ausgewählter Kundenkarten ... 23

Abb. 8: Definition und Hierarchie unterschiedlicher Ver-

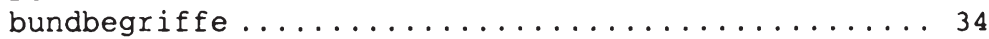

Abb. 9: Auf Nominalskalen basierende Verbundkoeffizienten ........................... 52

Abb. 10: Einflußgrößen des Kauf- und Nachfrageverbundes ...662

Abb.11: Typen von Verbundbeziehungen $\ldots \ldots \ldots \ldots \ldots \ldots \ldots$

Abb.12: Symmetrischer und asymmetrischer Verbund ......88

Abb.13: Vergleich von Kaufhäufigkeiten bei Kundenkartenbesitzern und Nicht-Kartenbesitzern ............94

Abb.14: Ablaufdiagramm Kaufverbundanalyse........... 100

Abb.15: Häufigkeitsmatrix von Verbundkäufen ......... 101

Abb.16: Abteilungsprofile der Abteilungen A,B,C für die

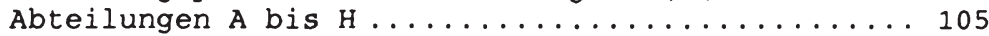

Abb.17: Ablaufdiagramm Nachfrageverbundanalyse ........ 107

Abb. 18: Ablaufdiagramm Zielgruppenbildung .......... 112

Abb.19: Altersverteilung in der Gesamtstichprobe ...... 116 Abb.20: Einkommensverteilung in der Gesamtstichprobe .... 117

Abb.21: Verteilung von Käufen in den Altersklassen ...... 127

Abb.22: Dendrogramm der Kaufverbundanalyse.......... 131 
Abb.23: Faktoren mit ihren bestimmenden Abteilungen in der Grundgesamtheit .................... 144

Abb.24: Gemeinsamkeiten zwischen Faktoren in den Untergruppen und Faktoren der Grundgesamtheit ....... 157

Abb.25: Entscheidungsbereiche des Handels mit Bezug zur Verbundmessung ..................... 182

Abb.26: Zuordnungsvergleich von Abteilungen $\mathrm{zu}$ Geschäftsbereichen und verbundclustern ......... 187

Abb.27: Ermittlung eines Kundenweges anhand eines Kaufdatensatzes ......................... 197

Abb.28: Häufigkeitsmatrix der Kundenbewegungen ........ 198 


\section{TABELLENVERZEICHNIS}

Tab. 1: Zuordnung von Abteilungen zu Geschäftseinheiten . . 120

Tab. 2: Zuordnung von Abteilungen zu Stockwerken ....... 139 
VERZEICHNIS DER ABKÜRZUNGEN

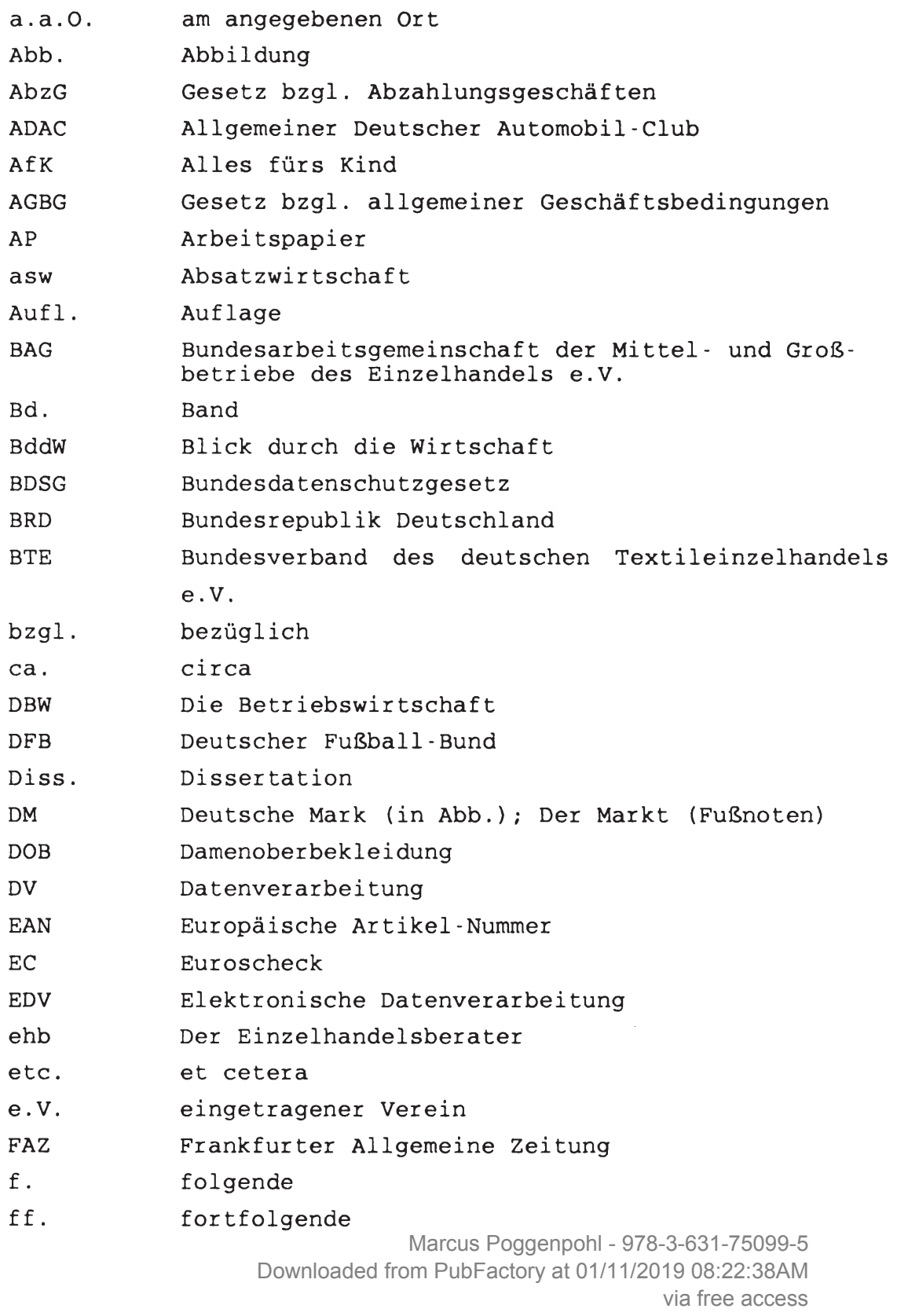




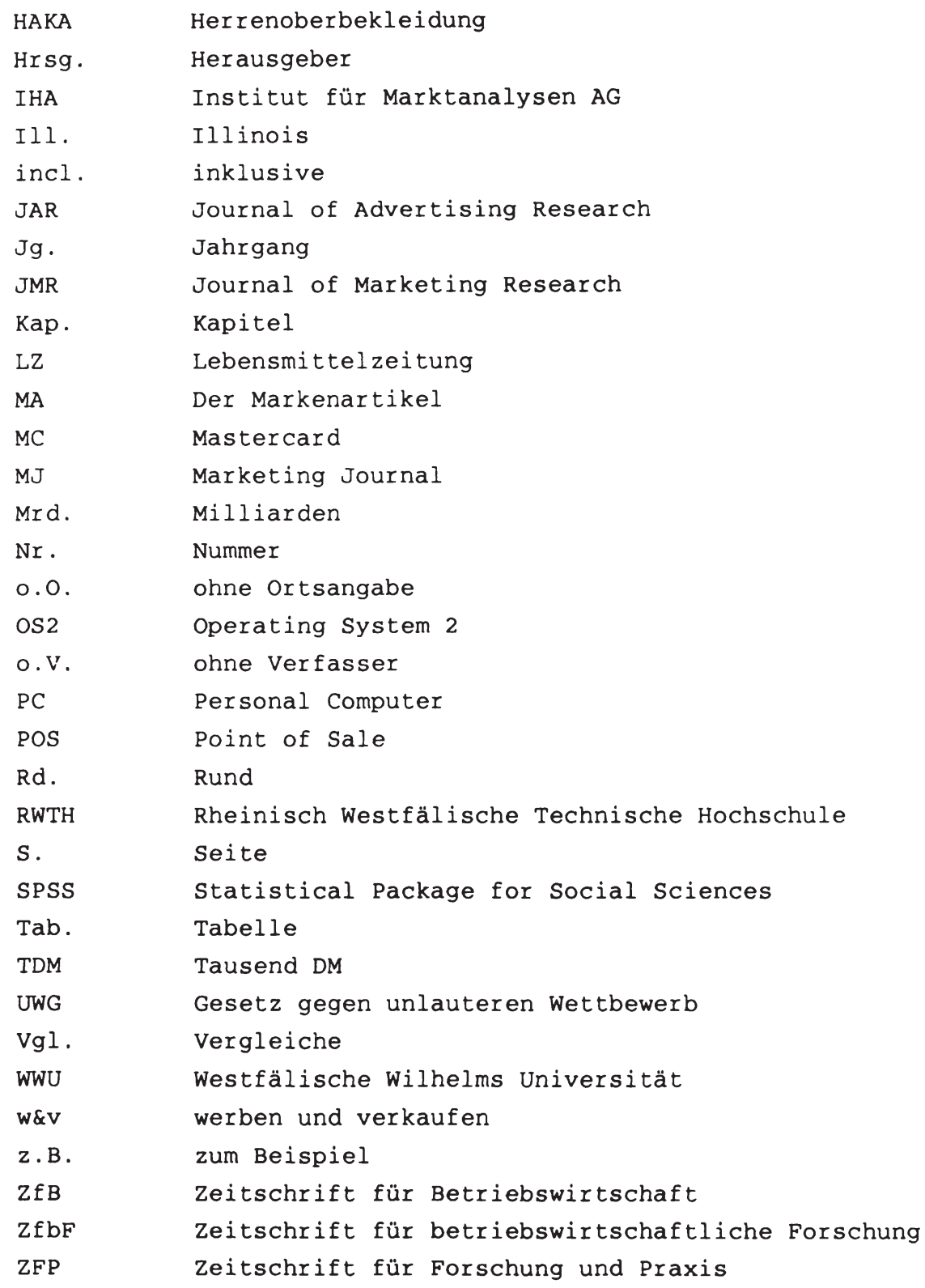


Marcus Poggenpohl - 978-3-631-75099-5

Downloaded from PubFactory at 01/11/2019 08:22:38AM

via free access 


\section{A. Kundenkarteninformationen als Grundlage der Verbund- analyse im Einzelhandel}

\section{Die Bedeutung von Verbundanalysen für das Handelsmarke- ting}

Seit Anfang der 80er Jahre sind Handelsunternehmen mit einer zunehmend dynamischen und komplexen Unternehmensumwelt konfrontiert. Das Aufkommen und die Aktivitäten neuer Anbieter, eine steigende Ausstattung des Haushalts mit Ge- und Verbrauchsgütern sowie Schwächen in der konjunkturellen Entwicklung haben vor diesem Hintergrund $\mathrm{zu}$ einer Wettbewerbssituation geführt, die von Marktsättigung und -verdrängung gekennzeichnet ist. ${ }^{1}$ In diesem zusammenhang besteht für die Handelsunternehmen die Notwendigkeit, Maßnahmen zur sicherung des Unternehmenserfolges $\mathrm{zu}$ ergreifen.

Für ein Handelsunternehmen stellt die Zusammensetzung des Sortiments eine zentrale Ausgangsbasis für sein unternehme-

1 Vgl. insbesondere zur Marktsättigung und strategien in stagnierenden Märkten: Meffert, H., Marktorientierte Führung in stagnierenden und gesättigten Märkten, Arbeitspapier $\mathrm{Nr}$. 9 der Wissenschaftlichen Gesellschaft für Marketing und Unternehmensführung e.V., Meffert, H., Wagner, H. (Hrsg.), Münster 1983, S. 2 ff.; Meffert, H., Wettbewerbsorientierte Marketingstrategien im Zeichen schrumpfender und stagnierender Märkte, in: strategisches Marketing, Raffée, H., Wiedmann, K.-P. (Hrsg.), Stuttgart 1989, S. $475 \mathrm{ff}$; Meffert, H., Strategische Planungskonzepte in stagnierenden und gesättigten Märkten, in: Die Betriebswirtschaft, 1983, S. 193 ff.; Meffert, H., Marketingstrategien in stagnierenden und schrumpfenden Märkten, in: Betriebswirtschaftliche Entscheidungen bei stagnation, Edmund Heinen zum 65 . Geburtstag, Pack, L., Börner, D. (Hrsg.), Wiesbaden 1984, S. $32 \mathrm{ff.;}$ Meffert, H., Walters, M., Anpassung des absatzpolitischen Instrumentariums in stagnierenden und schrumpfenden Märkten, in: Betriebswirtschaftliche Beiträge zur Bewältigung der ökonomischen Krise, Staehle, K.H., Stoll, E., Wiesbaden 1984, S. 141 ff; KroeberRiel, W., Zentrale Probleme auf gesättigten Märkten, in: Marketing ZFP, 3/1984, S. 210 ff. 
risches Handeln dar. ${ }^{2}$ Dabei steht aber nicht nur die Problematik der Warenbeschaffung im Vordergrund. Von zunehmender Bedeutung für einen wirtschaftlichen Erfolg der Handelsunternehmen ist die Anpassung der Sortimentsgestaltung an die Anforderungen der Konsumenten.

Um die Sicht der Konsumenten bzgl. der Sortimentszusammensetzung $z u$ verstehen, ist eine einfache Analyse von Abverkaufszahlen nicht ausreichend. Es ist notwendig, Verbindungen und kausale Beziehungen zwischen Käufen verschiedener Produkte oder in unterschiedlichen Sortimentsbereichen aufzudecken. Nur auf diese Weise wird eine umfassende Dokumentation der Anforderungen von Konsumenten an die Sortimentszusammenstellung ermöglicht. Letztendlich sollen die Erkenntnisse als Grundlage zur Effizienzsteigerung absatzpolitischer Maßnahmen dienen. 3

Zur Aufdeckung von Beziehungen zwischen den Käufen verschiedener Produkte trägt die Verbundforschung bei. Erste empirische Ansätze zur Erfassung von Verbundbeziehungen entstanden in den 70er Jahren. Unter Verbundanalyse wird die Untersuchung der Beziehungen und Wirkungen einzelner Sortimentsbestandteile in bezug auf ihre gegenseitigen Nachfragesynergien verstanden. Den Oberbegriff für diese allgemeinen Wirkungen bildet der Sortimentsverbund.

2 Zum sortiment als zentraler Bestandteil einer strategischen Erfolgsposition vgl. Meffert, H., Erfolgsfaktoren im Einzelhandelsmarketing, in: Marketing-Erfolgsfaktoren im Handel, Bruhn, M. (Hrsg.), Frankfurt/Main, New York 1987 , S. $20 \mathrm{f}$.

Vgl. Merkle, E., Die Erfassung und Nutzung von Informationen über den Sortimentsverbund in Handelsbetrieben, in: Schriften zum Marketing, Dichtl, E., Böcker, F. (Hrsg.), Bd. 11, Berlin 1981, S. 1. 
1) "Unter sortimentsverbund versteht man allgemein alle einseitigen und gegenseitigen Ausstrahlungseffekte zwischen einzelnen Sortimentsteilen; dabei können die Interdependenzen bzw. Dependenzen sowohl durch Gegebenheiten der Nachfrage als auch des Angebots bedingt sein." 4

Im Rahmen der vorliegenden Untersuchung sind darüber hinaus

der Kauf- und Nachfrageverbund von besonderer Bedeutung:

2) Unter Raufverbund werden die in einem Kauf $z u$ einem zeitpunkt realisierten sortimentsverbundbeziehungen verstanden.

3) Der Nachfrageverbund bildet als zeitraumbezogene Größe alle Käufe eines Individuums oder Haushaltes innerhalb eines vordefinierten Zeitraums ab.

Die Untersuchung des Kaufverbundes kann von den Handelsunternehmen für unterschiedliche zwecke genutzt werden:

- Bei der Untersuchung bestehender Sortimente stehen Ausweitungs- und Eliminationsentscheidungen auf Artikeloder Warengruppenebene im Vordergrund. Zudem sind Hinweise auf die Notwendigkeit von Veränderungen der Sortimentsstruktur möglich. Diese Hinweise ergeben sich z.B. aus einer verbundbezogenen Deckungsbeitragsrechnung. 5

4 Böcker, F., Die Bestimmung der Kaufverbundenheit von Produkten, in: Schriften zum Marketing, Dichtl, E., Böcker, F. (Hrsg.), Bd. 7, Berlin 1978, S. 19; vgl. Engelhardt, W.H., Erscheinungsformen und absatzpolitische Probleme von Angebots- und Nachfrageverbunden, in: ZfbF 1976, S. 77 ff.; Zeichen, A., Die Darstellung der Auftragsgrößenstruktur und die Möglichkeiten ihrer Veränderung im Absatzbereich der Unternehmung, in: Die Unternehmung 1974 , S. 209.

5 Vgl. Merkle, E., Die Erfassung und Nutzung von Informationen über den Sortimentsverbund in Handelsbetrieben, a.a.0., S. 161 f.; Sabel, H., Produktpolitik in absatzwirtschaftlicher sicht, Grundlagen und Entscheidungsmodelle, Wiesbaden 1971, S.M176s ffogenpohl-978-3-631-75099-5 
- Ergebnisse von Kaufverbundanalysen können auch für preispolitische Entscheidungen genutzt werden. ${ }^{6}$ wenn $z \cdot B$. ein starker Kaufzusammenhang zwischen zwei Produkten besteht, kann durch eine Preissenkung bei einem Produkt trotz der dadurch geringeren Gewinnspanne aufgrund der verbundbedingten Absatzsteigerung des zweiten Produktes eine Ertragssteigerung erzielt werden. In diesem Bereich bestehen bisher nicht gelöste Meßprobleme, die sich insbesondere auf die Isolierung von kausalen Verbindungen beziehen, wenn verschiedene Preisfestlegungen beim Kauf von Artikelkombinationen betrachtet werden. 7

- Zur steuerung von werbepolitischen Maßnahmen z.B. bei der Auswahl zu bewerbender Artikel oder zur Zusammenstellung sinnvoller Angebotspakete können Verbundanalysen ebenfalls wertvolle Anregungen geben. Dabei wird die Zusammenstellung durch die stärke des Verbundes determiniert. 8

- Schließlich können Verbundanalysen auch zur Überprüfung des Erfolges von Werbeaktionen eingesetzt werden, indem nicht nur der Abverkauf von beworbener Ware erfaßt wird. Darüber hinaus werden auch noch alle zusätzlich bei diesem Kaufakt getätigten Artikelkäufe ermittelt. Dadurch ergibt sich die Verbundwirkung einer Werbeaktion. Dabei wird eine Kaufbeziehung postuliert, bei der das Werbemittel auslösender Faktor ist. Der Kauf der Werbeware wird dann als Manifestation der durch das Werbemittel ausgelösten Kaufentscheidung angesehen.

Vgl. Simon, H., Preismanagement, wiesbaden 1982, S. 418.

7 Vgl. Heidel, B., Die Nutzung von Scannerdaten zur Planung der Absatzpolitik von Einzelhandelsbetrieben, a.a.0., S. 112 .

Vgl. Diller, H., Die wirkung von Verkäufen unter Einstandspreisen im Lebensmitteleinzelhandel, in: DBW, 41. Jg. 1981 , S. 409-418. 
- Besonders geeignet sind die Ergebnisse von Verbundanalysen auch für die Planung der Plazierung von Artikeln im Rahmen der Regalplanung oder von Abteilungen und Warengruppen bei der Geschäftsstättenplanung. Durch eine unterschiedliche Plazierung von warengruppen mit hohem verbund läßt sich z.B. die Aufenthaltsdauer und Kontaktstrecke eines Kunden im Geschäft beeinflussen. Dies kann zur Minimierung des Zeitaufwandes für den Kunden, aber auch zur Maximierung der Aufenthaltsdauer genutzt werden.

Zur Gewinnung einer Datenbasis für die empirischen Untersuchungen zum Verbund konnten bisher drei verschiedene Informationsinstrumente benutzt werden:

- Beobachtung

- Befragung

- Sammlung von Kaufdaten (Kassenbelege).

Die Beobachtung und die Befragung sind jedoch als Informationsquellen für detaillierte Verbundanalysen aufgrund des notwendigen umfangreichen Datenmaterials eher ungeeignet. Darüber hinaus sind beide Verfahren nur mit erheblichem Aufwand durchführbar. Im Bereich der Befragung treten zudem noch Intervieweffekte auf, die zu Verzerrungen der Ergebnisse führen können.

Die Auswertung von Kassenbelegen stellt vor allem beim Einsatz von Scannerkassen einen erheblichen Fortschritt für die Datenanalyse der Verbundforschung dar. Doch fehlen dabei jegliche Bezüge zu Personendaten von Konsumenten. Daher sind auch bei dieser Datenbeschaffungsmethode die Möglichkeiten der Verbundanalysen eingeschränkt. Letztlich erscheint es notwendig, ein neues Instrument der Informationsbeschaffung zu nutzen, um die vorhandenen Defizite auszugleichen. In diesem zusammenhang stellt die Verwendung von Informationen, 
die bei der Nutzung von Kundenkarten im Handel entstehen, einen Ansatzpunkt dar. Daraus können für die Verbundforschung neue Anwendungsbereiche erschlossen und die bisherigen Analysemethoden qualitativ verbessert werden.

\section{Die Entwicklung des Rredit- und Rundenkartenmarktes in der Bundesrepublik unter besonderer Berücksichtigung des Einzelhandels}

In den vergangenen Jahren hat in Deutschland eine Entwicklung eingesetzt, die sich in den USA bereits seit Mitte der 70er Jahre vollzieht und die sich in einer zunehmenden Substitution der Bargeldzahlung durch bargeldloses Zahlen mittels Kreditkarten und anderer Bargeldsubstitute äußert. 9 so hat sich der Kreditkartenmarkt in Deutschland von ca. 500.000 ausgegebenen Karten zu Beginn der 80er Jahre bis auf 5,5 Millionen Karten im August 1991 entwickelt. Wachstumsprognosen lassen bis Mitte der 90er Jahre 10 Millionen Karten erwarten. 10

In Deutschland wurde diese Entwicklung vor allem durch die Euroscheckkarte und das europaweit einzigartige system des Kontokorrentkredits verzögert. Dadurch war der Bedarf an anderen bargeldlosen Zahlungsmitteln eher gering. Besitzt bereits jeder amerikanische Haushalt zumindest eine Kreditkarte, so sind in Deutschland die Marktpotentiale für dieses Bargeldsubstitut bei weitem nicht ausgeschöpft. 11

9 Vgl. z.B. Böhme, K., Geldkartensysteme auf neuen wegen verpaßte Gelegenheiten oder neue Chancen?, in: bank und markt, 3/1988, S. 6 ff.

10 Vgl. o.v., Hertie und Barclays bauen optimus Bank aus, in: FAZ vom 15.08.1991, S. 12 .

11 Dies lassen die schätzungen über die kreditkartenverbreitung in anderen Ländern erwarten. Hiernach sind z.B. in Großbritannien 38 Millionen, in Frankreich 12 MillioMarcus Poggenpohl - 978-3-631-75099-5 
Gleichwohl haben die intensiven Bemühungen der Kreditkartengesellschaften um die Potentiale des europäischen Marktes auch in Deutschland $z u$ einer steigenden Anzahl von Kreditkartenbesitzern geführt. Der Markt zeigt eindeutige Wachstumstendenzen. Abbildung 1 verdeutlicht, daß die Kreditkartenumsätze in Deutschland über einen Zeitraum von drei Jahren um mehr als $70 \%$ gestiegen sind. Dabei gingen starke Wachstumsimpulse vor allem von vergleichsweise günstigen

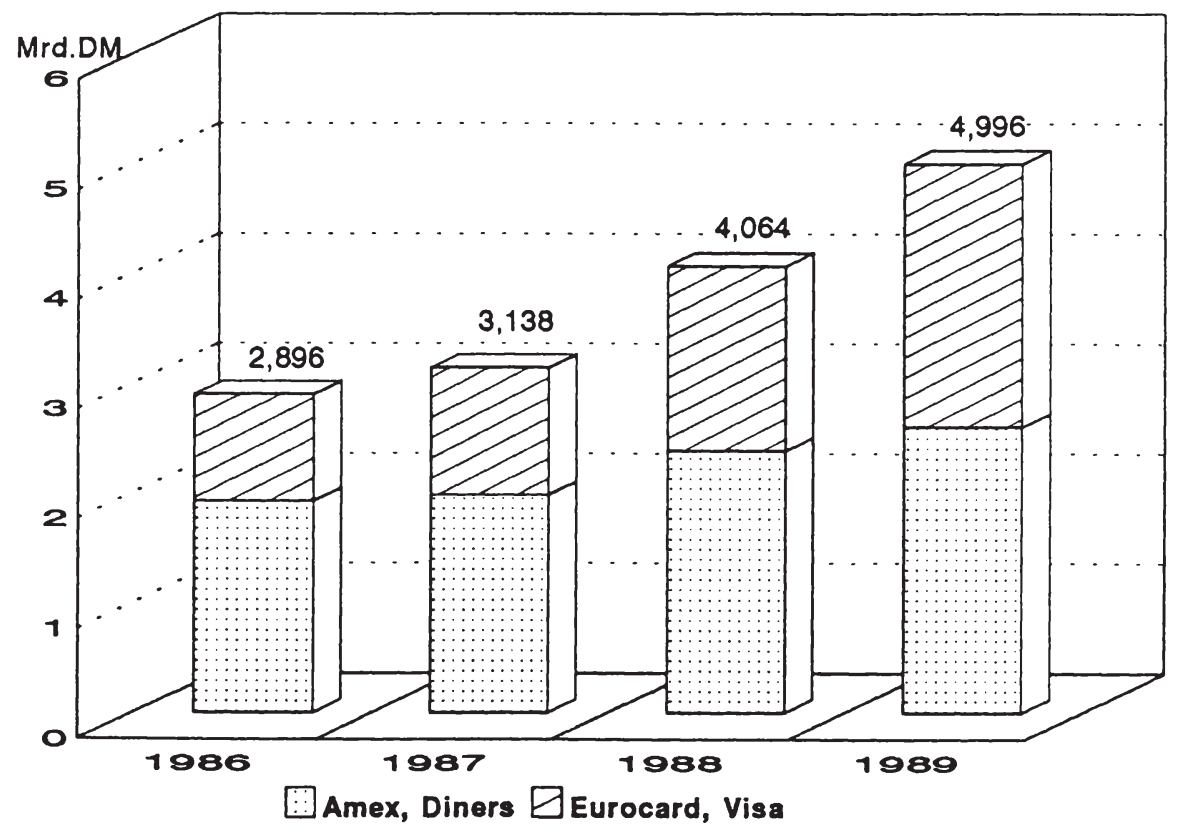

Abb. 1: Kreditkartenumsätze in der BRD 1986-1989 (ohne Warenhausumsätze) (Quelle: o.V., Nielsen Report 1989, Frankfurt 1990, O.S.)

nen und in den USA 320 Millionen Karten im Umlauf. Somit ist noch ein großes ungenutztes Marktpotential zu vermuten, auch wenn amerikanische Verhältnisse nicht erreicht werden (z.B. wegen des Euroscheck-Systems). Vgl. zu den Schätzwerten $0 . V .$, Hertie und Barclays bauen optimusBank aus, a.a.0., S. 12. Marcus Poggenpohl-978-3-631-75099-5 
Kreditkarten (Euro- und Visa Card) aus. 12 Dies wird insbesondere dadurch begünstigt, daß sich die Kreditkarte vom statusobjekt zum Massenzahlungsmittel entwickelt. Die ständig fallenden Kosten für den Erwerb von Kreditkarten fördern diese Tendenz.

Demgegenüber stellt das im Aufbau befindliche EC-Cash-System $^{13}$, das kreditkartenähnliche Zahlungsmöglichkeiten für EC-Karten gewährt, einen möglichen Begrenzungsfaktor für die Diffusion von Kreditkarten dar. Dies gilt vor allem, da in diesem Fall bereits 23 Millionen kreditkartenähnliche $\mathrm{Zah}-$ lungsmittel im Umlauf sind. 14

Darüber hinaus hat im Kreditkartenmarkt die Ausgabe von Kreditkartensubstituten durch den Einzelhandel zu einer substitution von Kreditkartenumsätzen durch Kundenkartenumsätze geführt. 15 In den USA haben die Umsätze von Handelskarten die Kreditkartenumsätze im Handel bereits überflügelt. $16 \mathrm{Ab}-$ bildung 2 verdeutlicht diese Entwicklung am Beispiel der USMarktführer im Warenhausbereich.

12 Vgl. o.v., Dezentral macht stark, in: w\&v, Nr. 44/2, Nov. 1990 , S. 14 .

13 Beim EC-Cash-System kann jeder Besitzer einer Euroscheck-Karte mit PIN-Code (Geheimnummer) ohne Euroscheck bargeldlos zahlen.

14 Vgl. Heiner, V., Tankstellen Vorreiter für Handel Großversuche mit dem neuen "Electronic Cash"-System, in: Dynamik im Handel, 12/1990, S. 12 ff.; Klein, S., Electronic Cash-System: Langer Weg mit Hindernissen, in: à la card aktuell, Nr. 2B/1990, S. $6 \mathrm{ff}$.

$15 \mathrm{Zu}$ den Zielen einer solchen substitution vgl. Kapitel A.2. dieser Untersuchung.

16 Vgl. Poynor Burns, R., Discover Card: An Industry Surprise, in: Cards International, $29 / 1 / 1990$, S. $13 \mathrm{ff}$. 


\section{Zahlungssysteme in US-Handelsunternehmen}

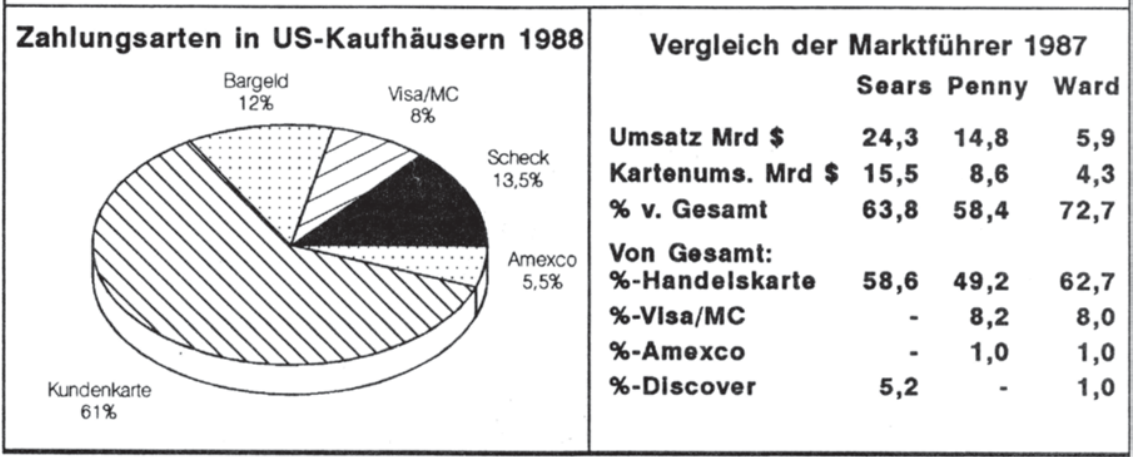

Abb. 2: Anteile von Zahlungsarten in US-Handelsunternehmen (Quelle: 0.V., Nielsen Report 1988, Frankfurt 1989, o.S.; O.V., zucker für Treulose, in: DM, 2/1991, S. 62)

Die Ausgabe handelseigener Karten durch eine steigende Anzahl von Handelsunternehmen trägt jedoch dazu bei, daß auf dem Markt eine nahezu unüberschaubare vielfalt von Karten mit unterschiedlichsten Bezeichnungen und Ausstattungsmerkmalen entsteht, die dem Konsumenten die orientierung erschwert. 17 Eine mögliche strukturierung dieses Kartenangebots ist Abbildung $3 \mathrm{zu}$ entnehmen. 18

17 Zur Ausstattung und Gestaltung von Karten auch in Abbildung 7 vgl. z.B. Zellekens, H.J., Fontaine, J., Braatz, F., Zahlung per Karte, Köln 1989, S. 13 ff.; 0.V., Kundenkarten im Einzelhandel, BTE Fachdokumentation, Köln 1990; 0.V., Die Karten werden neu gemischt, in: test $2 / 90$, S. 16; 0.V., à la card, 2. Aufl., Hamburg 1989; o.V., Was Kundenkarten bieten, in: DM, 2/1991, S. 63 .

$18 \mathrm{Vgl}$. $\mathrm{zu}$ anderen strukturierungen z.B. Mohme, J., Der Einsatz von Kundenkarten im Einzelhandel - Konzeptionelle und praktische Probleme kartengestützter Kommunikationssysteme und Kundenbindungsstrategien im stationären Einzelhandel, Diss. Münster 1991, S. 13 ff.; zur Problematik des Zwei- bzw. Drei-Parteien-systems (Karteninhaber als erste Partei und Kartenemittent/Vertragspartner als zweite Partei bzw. jeweils als Einzelpartei Karteninhaber und Kartenemittent und Vertragspartner) vgl. Ahlert, D., Schröder, E., Rechtliche

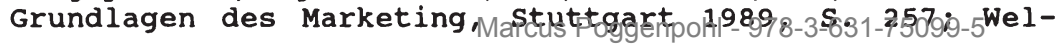




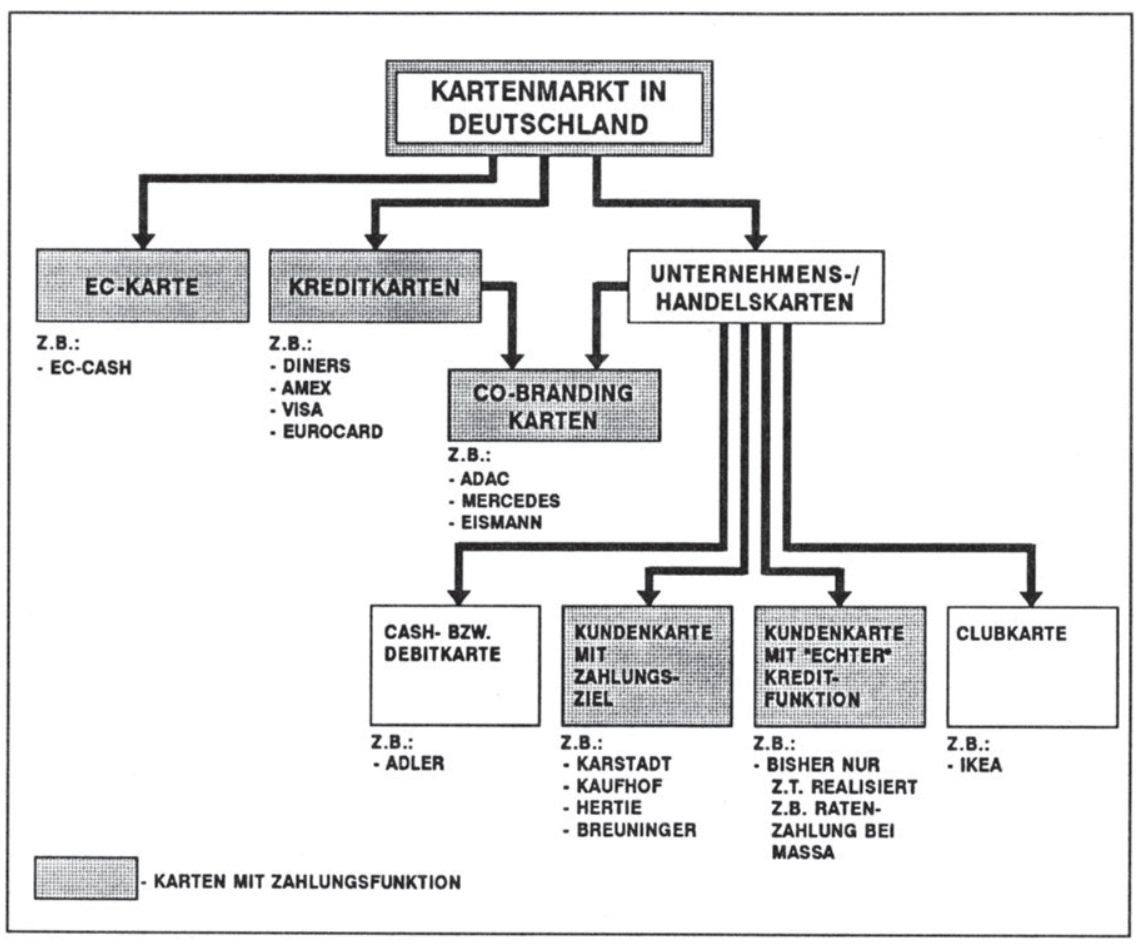

Abb. 3: Strukturierung von Kartensystemen in Deutschland Im deutschen Kreditkartenmarkt bestehen neben dem EC-CashSystem die klassischen Kreditkarten, die auch einen echten Kreditrahmen schaffen. ${ }^{19}$ Die von Handelsunternehmen ausgegebenen Karten gewährleisten dies meist nicht. ${ }^{20}$ Darüber hin-

ler, M., Das Kreditkartenverfahren, Köln 1986, S. 46 ff.; Kundenkarten sind dabei überwiegend als Zwei-Parteien-System aufgebaut, da das Handelsunternehmen gleichzeitig als Kartenemittent und Vertragspartner (Akzeptanzstelle) auftritt.

19 Eine echte Kreditfunktion setzt voraus, daß fällige Auslagenerstattungen durch den Kartenemittenten in eine Darlehensgewährung $z u$ bestimmten Zinssätzen umgewandelt wird; vgl. v. Usslar, L., v. Morgen, R.D., Aktuelle Rechtsfragen der Kreditkarten-Praxis, Hamburg 1989, S. 33 .

$20 \mathrm{Zu}$ den unterschiedlichen Ausstattungen von Handelskarten vgl. Kapitel A.2. 
aus hat sich eine Zwischenform herausgebildet, die als coBranding-Rarte bezeichnet wird. 21 Dabei kooperiert ein Kartenanbieter mit einem Unternehmen, das eine eigene Karte emittieren will.22 Die Karte trägt dann sowohl ein Kreditkartenlabel als auch die Kennzeichnung des ausgebenden Unternehmens. 23 ziel der kreditkartenunternehmen ist eine schnellere Diffusion ihrer Kreditkarten, während das ausgebende Unternehmen eine eigene Karte mit echten Kreditkartenfunktionen zu geringen Kosten anbieten kann. 24

Der Schwerpunkt der vorliegenden Untersuchung liegt im Bereich der reinen Handelskarten, da nur durch die eigenständige Emission, Abwicklung und Kontrolle der Karte Informationen über den Konsumenten und seine Verbundkäufe in vollem Umfang für das Handelsunternehmen nutzbar sind. Diese Handelskarten werden überwiegend von filialisierten Handelsunternehmen ausgegeben, da nur dort ein ausreichendes Kundenpotential besteht.

Bereits anhand der Grundfunktionen lassen sich drei grundsätzliche Kundenkartentypen gegeneinander abgrenzen Abbil-

21 In der Literatur ist der Begriff der co-branding-Karte nicht einheitlich definiert. Vgl. Usslar, L., v. Morgen, R.D., a.a.O., S. 120; Hüttermann, M.U., Formen und Risiken des Co-branding, Hamburg 1991; Michalek, M., Cobranding: Chance oder Bedrohung, in: à la card Aktuell, Nr. 12/1991, S. $11 \mathrm{f}$.

22 Vgl. o.V., EC-Karte und Geheimzahl: Genügt das ...?, in: ehb $10 / 90$, S. 767 f.

23 Hauptpartner ist dabei Visa; vgl. o.V., Dezentral macht stark, a.a.O., s. 14 .

24 In der praxis konnten sich die co-branding-Karten bisher kaum durchsetzen (2.B. ADAC-Visa, Eismann-Visa; DFB-Visa etc.). Vgl. o.V., Co-branding: Die Luft ist raus, in: à la card Aktuell, Nr. $12 / 1991$, S. 6 ff.; 0.V., Handelskarten: Orden für Stammkunden, in: à la card Aktuell, Nr. 11/1991, S. 37; Hermann, T., Co-branding: Sieche Patienten, in: à la card Aktuell, Nr. 12/1991, S. 15. 
dung 4 zeigt eine Einordnung der drei Typen anhand ihrer Nutzenbeiträge für das Unternehmen und die Konsumenten.

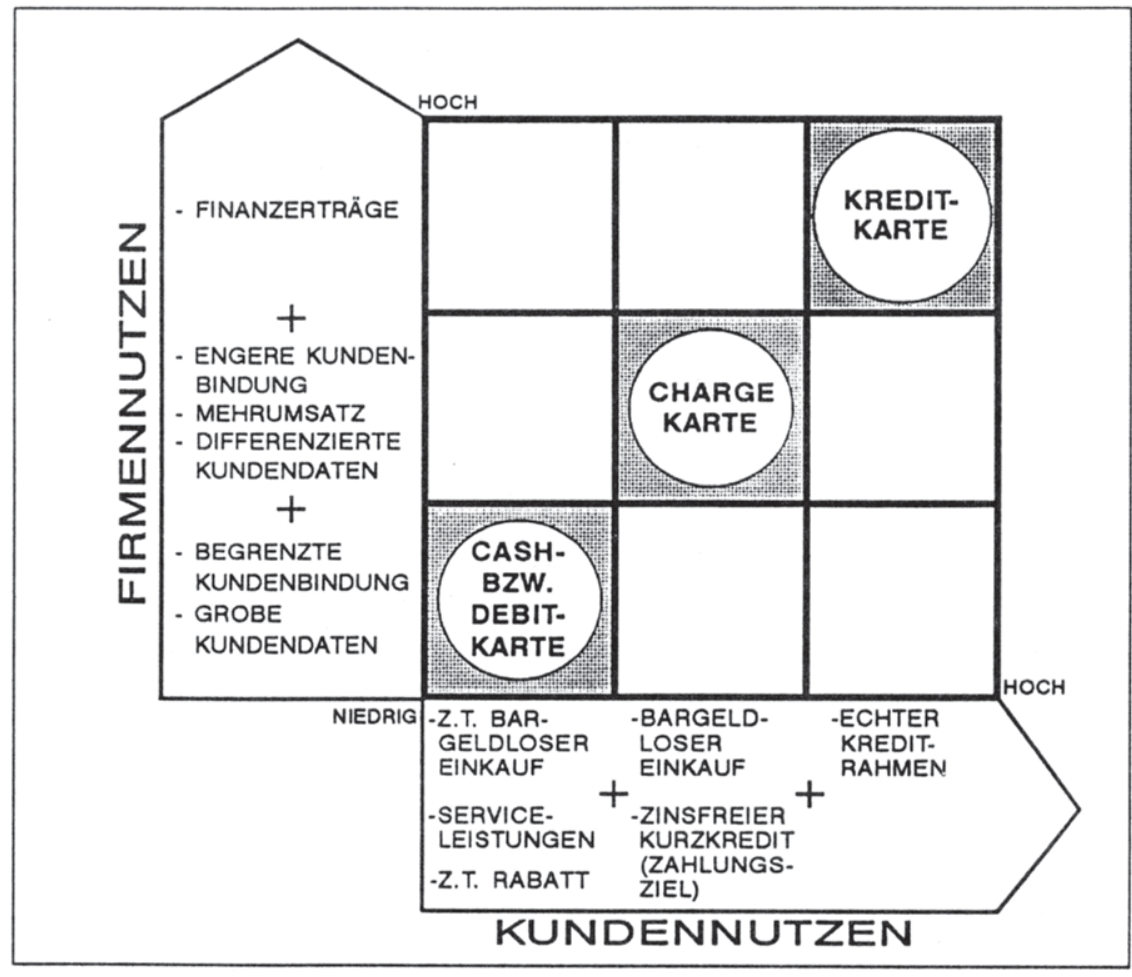

Abb. 4: Positionierung von Kundenkartentypen in einer Nutzenmatrix

- Die Cash- bzw. Debit-Rarte: Bei einer Cash- bzw. DebitKarte steht die Ausweisfunktion im Vordergrund. Sie kann allerdings mit einem Rabattsystem ${ }^{25}$ gekoppelt sein. Für das Handelsunternehmen ergibt sich dadurch lediglich eine begrenzte Kundenbindung. Zudem ermöglicht dieser Karten- 
typ nur die Erhebung fragmentarischer Daten ${ }^{26}$ für spätere Analysen. Unter diesen Kartentyp fallen auch die Kartensysteme, die beim bargeldlosen Zahlungsverkehr direkt bzw. mit geringfügigen Verzögerungen das Konto des Kunden belasten (z.B. EC-Cash). Bei einer reinen Ausweisfunktion würde dieser Kartentyp aufgrund der Kundenkartendefinition nicht mehr zu den Kundenkarten zu zählen sein.

- Chargekarte: Diese Karte repräsentiert den heute gebräuchlichsten Typ von Kundenkarten (z.B. Hertie, Kaufhof). Mit dieser Karte ist ein bargeldloser Einkauf möglich, wobei dem Kunden ein zinsfreier Kurzkredit gewährt wird. Die Abrechnung erfolgt meist nach ca. 6 Wochen. Für das Handelsunternehmen ergeben sich eine engere Kundenbindung sowie differenziertere Kundendaten, z.B. die Speicherung jedes Kaufs des Kartenbesitzers. Darüber hinaus werden umfangreichere soziodemographische Daten erfaßt als bei der Debitkarte. Zudem durchlaufen die demographischen Kundenangaben eine Prüfroutine.

- Rarte mit echter Rreditfunktion: Dieser Kartentyp wird bisher nur sehr begrenzt eingesetzt 27 , da er eine Kooperation mit einer Bank oder zumindest eine eigene qualifizierte Kreditabteilung voraussetzt. Die Banken schätzen aber häufig die Risiken derartiger ungeprüfter Verbraucherkredite als zu hoch ein. Dem Kunden wird ein "echter" Kontokorrentkredit mit banküblichen konditionen gewährt. Es werden somit Kreditkartenkonditionen beschränkt auf ein Handelsunternehmen angeboten. Für das Handelsunter-

26 Es werden keine Kaufdaten gespeichert, und die Erfassung demographischer Daten ist rudimentär. Die Angaben werden zudem nicht geprüft.

27 z.B. Ratenzahlung bei Massa. Im Rahmen der vorliegenden Untersuchung werden ausschließlich reine Handelskarten betrachtet. Daher werden Co-branding-Karten nicht in die Betrachtung einbezogen. 
nehmen ergeben sich bei eigenständiger Kreditabwicklung Potentiale für Finanzerträge.

In der Literatur existiert keine einheitliche Definition des Begriffs Kundenkarte. Darüber hinaus wird die Kundenkarte z.T. durch weitere Begriffskonstrukte umschrieben. ${ }^{28}$ Daher wird die Kundenkarte im folgenden als ein Bargeldsubstitut verstanden, das als Identifikationsbeleg genutzt wird und dem Kundenkartenbesitzer bargeldloses Zahlen in den Geschäftsstätten des kartenemittierenden Unternehmens ermöglicht. Dabei tritt das kartenemittierende Unternehmen gleichzeitig als Vertragspartner bzw. Akzeptanzstelle auf.

Bei dieser Definition wird keine Vorgabe bzgl. einer echten Kreditfunktion oder eines Erfassungs- und organisationsmodus gestellt, um eine $z u$ enge Abgrenzung $z u$ vermeiden. Umfassende Verbundanalysen sind jedoch nicht ohne ein komplexes DV-System möglich. Daher sollte die technische Abwicklung der Karte in ein bestehendes bzw. aufzubauendes Warenwirtschaftssystem ${ }^{29}$ integriert werden. Dieses System

28 Z.B. Kundenkreditkarte, firmeneigene spezialkarte etc.; vgl. dazu Mohme, J., Der Einsatz von Kundenkarten im Einzelhandel, a.a.0., S. 20. Um eine konsequente strukturierung von Kundenkarten $\mathrm{zu}$ erreichen, werden Karten ohne Zahlungsfunktion aus der Definition ausgenommen. Zum einen werden mit diesen Kartentypen z.T. andere Ziele verfolgt. Zum anderen ist ohne Zahlungsfunktion keine automatische Erhebung von Kaufdaten garantiert. Ausgegrenzt sind u.a. Club-Karten (z.B. IKEAFamily-Card) und Rabattkarten (z.B. Adler-Card).

29 Zum Bereich Warenwirtschaftssystem vgl. Zentes, J., Tendenzen der Entwicklung von Warenwirtschaftssystemen, in: Zentes, J. (Hrsg.), Moderne Warenwirtschaftssysteme im Handel, Berlin 1984; Zentes, J., Warenwirtschaftssysteme - Auf dem Weg zum Scientific Management im Handel, in: Marketing ZFP, Heft 3/1988, S. 177-181; Ebert, K., Warenwirtschaftssysteme und Warenwirtschaftscontrolling, Schriften $\mathrm{zu}$ Distribution und Handel, Ahlert, D. (Hrsg.), Band 1, Frankfurt a.M. 1986; Sova, O., Piper, J., Computergestüzte Warenwirtschaft im Handel, Köln 1985 . 
ermöglicht eine Erfassung und Speicherung aller Daten und ihre Bereitstellung für eine DV-mäßige Weiterverarbeitung.

\section{Ziele, Ausgestaltungsformen und Einsatzbereiche von Kun- denkarten}

Die starke Verbreitung von Kreditkarten stellt vor allem den Handel vor neue Herausforderungen. Sowohl in den USA als auch in Europa steigt angesichts der Forderung der Kreditkartengesellschaften nach einem Disagio zwischen $3 \%$ und $7 \%$ vom Umsatz und steigender Kreditkartenumsätze der Anteil des Disagios an den Gesamtkosten laufend an.

Diesem rein finanzwirtschaftlichen Problem stehen auf der Nachfragerseite noch zusätzliche Entwicklungen gegenüber. Dazu zählt z.B. der Wertewandel des hedonistischen Konsumenten, der durch die Ausrichtung seines Handelns auf die Befriedigung physischer und psychischer Lustbestrebungen gekennzeichnet ist. ${ }^{30}$ Diese Bedarfs- und Bedürfnisstrukturen der Nachfrager haben nicht zuletzt dazu beigetragen, daB sich der Handel zunehmend an Erlebniswerten orientiert ${ }^{31}$ und

Vgl. Meffert, H., Der neue Konsument: Chance für das Marketing, in: IHA News, Heft $2 / 1986 . \mathrm{S} .12 \mathrm{ff} . ;$ Windhorst, K.-G., Wertewandel und Konsumentenverhalten, Münster 1985, S. 85 ff.; Trommsdorff, V., Wertewandel und Wandel im Handel, in: Trommsdorff, V. (Hrsg.), Handelsforschung 1986, S. 3 ff.; Gerken, G., Die Zukunft des Handels, in: MJ 4/1987, S. $300 \mathrm{ff}$.

31 Vgl. zu diesem Themenkreis z.B. Heinemann, G., Betriebstypenprofilierung und Erlebnishandel - Eine empirische Analyse am Beispiel des textilen Facheinzelhandels, Wiesbaden 1989; Ahlert, D., Evolutionäres Handelsmanagement - Die controllinggestützte Evolution erlebnisorientierter Betreibungskonzepte des stationären Einzelhandels, Arbeitspapier $\mathrm{Nr}$. 11 des Lehrstuhls für Betriebswirtschaftslehre, insbesondere Distribution und Handel der WWU, Münster 1990, S. I ff., Bost, E., Ladenatmosphäre und Konsumentenverhalten, Heidelberg 1987; Trommsdorff, V., Wertewandel und Wandel im Handel, in: 
eine Polarisierung in den Versorgungsbereich und den Zusatznutzenbereich festzustellen ist. 32 Darüber hinaus ist die sinkende Geschäftsstättentreue anzuführen. Sie führt insbesondere $\mathrm{zu}$ solchen Aktivitäten des Handels, die auf eine verstärkte Kundenbindung gerichtet sind. 33

Neben den verbraucherseitigen Herausforderungen muß sich der Handel insbesondere mit einer zunehmenden Wettbewerbsintensität auseinandersetzen, die zudem durch Konzentrationstendenzen überlagert wird. ${ }^{34}$ Dies führt $z u$ der wachsenden Notwendigkeit, sich gegenüber den Wettbewerbern zu profilieren.

Für das Handelsmarketing stellt sich die Frage, ob und in welchem Umfang die Ausgabe einer Kundenkarte zur Lösung der Probleme des Handels beitragen kann. Neben finanzwirtschaftlichen Zielsetzungen soll vor allem die Bindung des Konsu-

Handelsforschung 1986, Jahrbuch der Forschungsstelle für den Handel, Berlin (FFH) e.V., Trommsdorff, V. (Hrsg.), Heidelberg 1986, S. 3 ff.; Trommsdorff, V., Konsumentenverhalten, Stuttgart 1989, S. $150 \mathrm{ff.;}$ Raffée, H., Wiedmann, K.-P., Wertewandel und gesellschaftsorientiertes Marketing - Die Bewährungsprobe strategischer Unternehmensführung, in: Raffée, H., Wiedmann, K.-P., (Hrsg.), Strategisches Marketing, stuttgart 1989, S. 552 ff.; Stock, E., Wichert, R., Die Führungskraft im Handel als Kulturmanager, in: BddW, Nr. 93 vom 15.5.1990, S. 7; Ahlert, D., Schröder, H., "Erlebnisorientierung" im stationären Einzelhandel, in: Marketing ZFP, Heft 4/1990, S. $221 \mathrm{ff}$.

32 Vgl. Meffert, H., Marketingstrategien der Warenhäuser Wege aus der Krise?, in: Harvard Manager, Heft 2, 1985, S. 21 f.; O.V., Handel 2000, in: Sonderdruck der LZ, Nr. $52,30.12 .1982$, S. $1 \mathrm{ff}$.

33 Weinberg, P., Erlebnisorientierte Einkaufsstättengestaltung im Einzelhandel, in: Marketing ZFP, 2/1986, S. 97 ff.

34 Vgl. Böcker, F., Handelskonzentration: Abnehmende Unternehmenskonzentration bei zunehmender Entscheidungskonzentration, in: MA $6 / 1986$, S. $284 \mathrm{ff}$; Böcker, F., Handelskonzentration: Ein partielles Phänomen? - oder: Irreführende Handelsstatistiken, in: $\mathrm{ZfB}, 56 . \mathrm{Jg}$. Nr. $7 / 1986$, S. $654 \mathrm{ff}$. 
menten an das eigene Handelsunternehmen gestärkt werden. ${ }^{35}$ Aus Marketingsicht ist somit die Intensivierung der Kundenbindung das oberziel im Rahmen der Zielhierarchie ${ }^{36}$, während die Kreditkartensubstitution vornehmlich den Kostensenkungszielen dient. ${ }^{37}$ Eine Übersicht über das gesamte zielsystem der Ausgabe von Kundenkarten gibt Abbildung 5 .

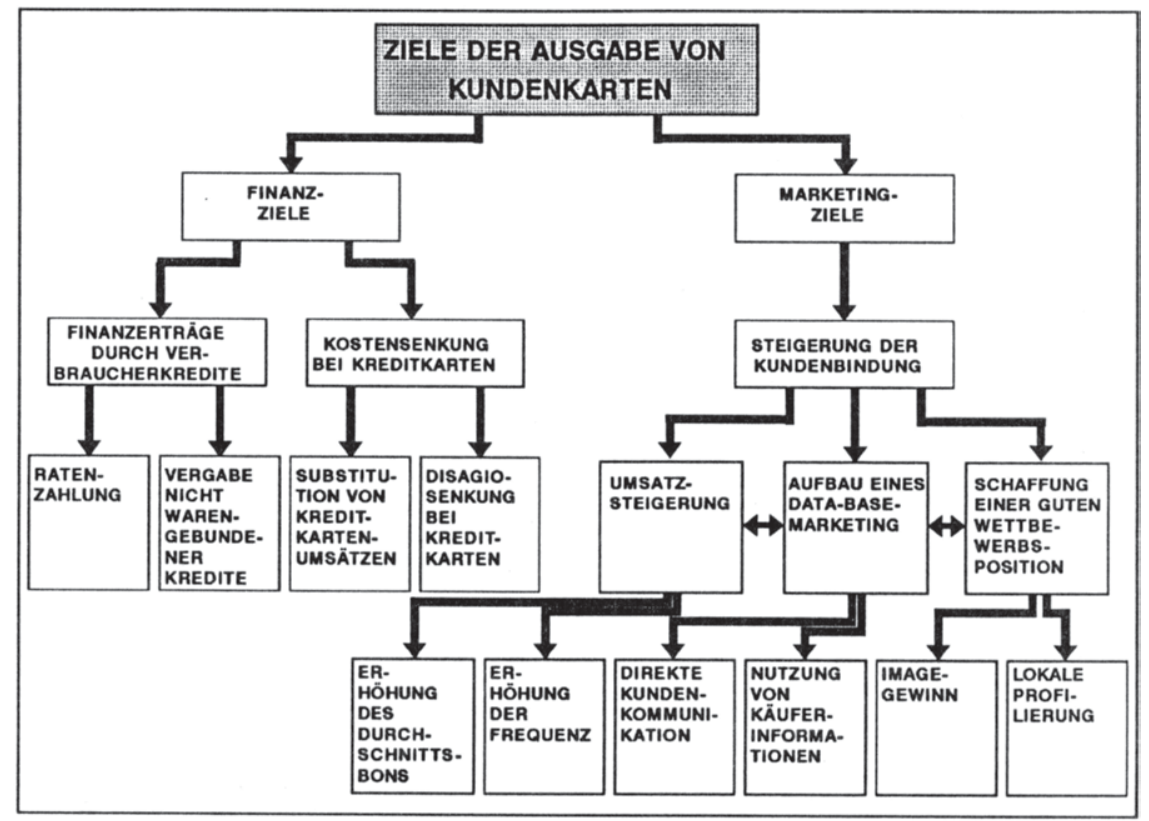

Abb. 5: Ziele der Ausgabe von Kundenkarten

Vgl. Kreuzer, R.T., Weppler, K., Marketing by Database. Eine Herausforderung für Markenartikler, in: Markenartikel 10/1990, S. 492 f.

Vgl. z.B. O.V., Im Dialog mit dem Kunden, in: Textilwirtschaft $9 / 1989$, S. 26.

37 Zu Zielen und Zielsystemen vgl. Kupsch, P., Unternehmensziele, Stuttgart, New York 1979; Heinen, E., Grundlagen betriebswirtschaftlicher Entscheidungen - Das Zielsystem der Unternehmung, 3. Aufl., in: Die Betriebswirtschaft in Forschung und Praxis, Heinen, E., Börner, D., Kappler, E., Kirsch, W., Meffert, H. (Hrsg.), Bd.1, Wiesbaden 1976. 
Durch die Gültigkeit der Kundenkarte allein im eigenen Handelsunternehmen soll die Bindung der Kunden an das Unternehmen erhöht werden. ${ }^{38} \mathrm{Um}$ diesen Effekt $\mathrm{zu}$ verstärken, werden bei Erwerb und Nutzung der Karte zahlreiche Zusatzleistungen angeboten. Diese Zusatzleistungen sollen darüber hinaus $z u$ einer verbesserten Diffusion der Kundenkarten bei den Kunden des Handelsunternehmens beitragen.

Gleichzeitig werden die Kundenkartenbesitzer intensiv beworben. Abbildung 6 zeigt die unterschiedlichen stufen einer Kundenbindung. Ziel des Handelsunternehmens ist es dabei, den Konsumenten gezielt von den unteren stufen der Kundenbindung bis zur intensiven Kundenbindung zu führen.39 Bei jeder Ausprägung der Kundenbindung ist dafür eine spezifische Gestaltung der Informationspolitik notwendig.

Die für das Handelsunternehmen verfügbaren Kundeninformationen bestehen in der untersten stufe lediglich aus grundlegenden Daten, die spezifische Erfordernisse einzelner Kundensegmente jedoch nicht berücksichtigen. Vor allem durch die Berücksichtigung von Kundenreaktionen z.B. auf Werbemaßnahmen oder Kaufangebote können zunehmend spezielle Interessengebiete der Kunden identifiziert werden. Auf Basis dieser Informationen ist dann eine an den Kundenproblemen ausgerichtete Bearbeitung der Kunden möglich, die sich bis zu einer permanenten Betreuung von stammkunden durch individuelle Direkt-Marketing-Maßnahmen entwickelt.

38 Vgl. Eggert, U., Bargeldlose Zahlungsmittel im Einzelhandel, in: Der Sport-Markt, 01/1989, S. 56; o.V., Mittel zur engeren Kundenbindung, in: Handelsblatt vom 09.08.1989, S. 11; 0.V., Mit der "Vertrauenskarte" Kunden binden, in: Textilwirtschaft Nr. 49, 12/1989, S. 12 .

39 Vgl. o.V., Die Basis für den Dialog, in: asw, 4/1990, s. 106. 


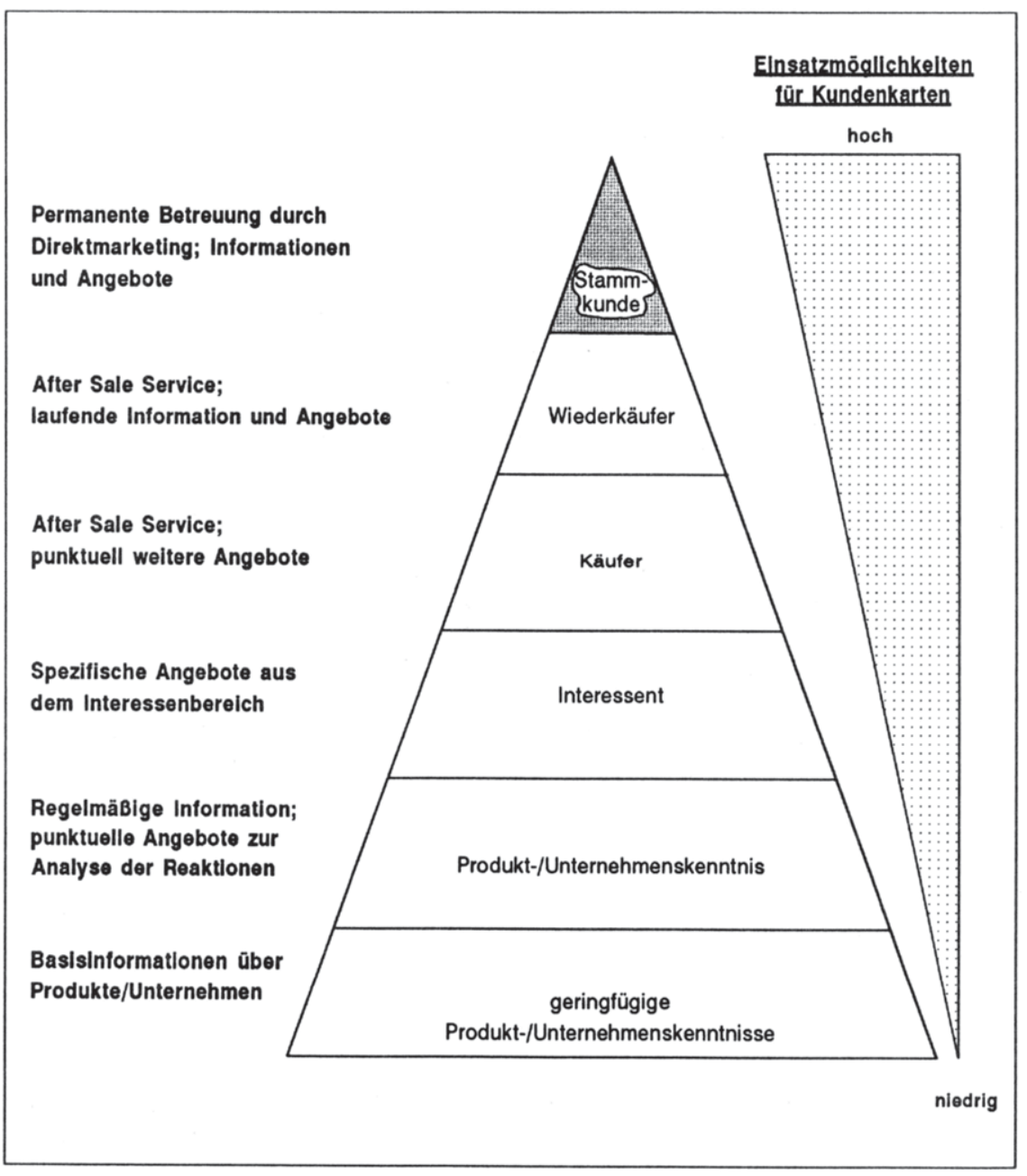

Abb. 6: Entwicklungsstufen der Kundenbindung (in Anlehnung an: o.V., Die Basis für den Dialog, in: asw, 4/1990, S. 105)

Die Intensivierung der Kundenbindung soll dabei zu einer Erhöhung der Besuchs- und Kauffrequenz führen. Dieser quantitative Anstieg der Besuche soll durch eine Erhöhung der Qualität der Besuche aus sicht des Handelsunternehmens ergänzt werden. Diese findet Ausdruck in einer steigerung des pqurch- 
schnittsbons eines Kunden. Letztlich führen diese beiden Wachstumspotentiale zu einer Umsatzsteigerung.

Durch die interne EDV-technische Abwicklung des Zahlungsverkehrs, der mit einer solchen Karte im Handelsunternehmen getätigt wird, werden gleichzeitig detaillierte Informationen über die Kunden gewonnen. Dabei eröffnen sich durch die Dokumentation von Kaufvorgängen für das Handelsunternehmen Informationspotentiale, die detaillierten Aufschluß über die Kunden geben. 40 Diese Informationen kann das Handelsunternehmen $z u$ einem qualifizierten Data-Base Marketing nutzen. Dabei ermöglichen die demographischen Angaben eine direkte Kundenansprache.41 Gleichzeitig wird die Erstellung zielgruppenspezifischer Angebote realisierbar.

Die Kundendaten liefern darüber hinaus detaillierte Informationen zur Fundierung weiterer Marketingentscheidungen. So sind z.B. Käuferstruktur-, Verbundkauf- sowie Sortimentsund standortanalysen möglich, die eine Entscheidungsunterstützung für Problemstellungen aus diesen Bereichen ermöglichen.

Ein weiterer Bestandteil der Zielhierarchie ist die Attraktivitätssteigerung des Handelsunternehmens, die zur schaffung einer gesicherten Wettbewerbsposition beitragen soll. Dabei steht ein Imagegewinn als kundenorientierter Partner im Vordergrund. Gleichzeitig wird eine Positionierung als innovativer Anbieter möglich.

Vor dem Hintergrund der zunehmenden Konzentration auf die lokalen Wettbewerbsbedingungen im Handel bietet die Kun-

40 Vgl. o.V., Königsweg zum Kunden?. in: asw 2/1991, S. 55 ff.

41 Vgl. O.V., Ihr Grundstein für eine treffsichere Direktwerbung, in: ehb, 1/1991, S. $42 \mathrm{ff}$. 
denkarte auch Möglichkeiten der lokalen Profilierung. Durch die standortdifferenzierte Auswertung der Informationen sowie die Einbindung lokaler Besonderheiten in die Aktionen des Kundenkartenmarketing entsteht ein standortspezifisches Bild filialisierter Handelsunternehmen. Eine derartige lokale Profilierung ist allerdings erst auf Basis eines umfangreichen Kundenstamms wirtschaftlich sinnvoll durchführbar.

Ähnlich wie bei Kreditkarten gibt es auch im Bereich der Kundenkarten eine vielfalt von Karten mit unterschiedlichen Ausstattungsmerkmalen durch die steigende Zahl von Handelsunternehmen, die die Ausgabe von Kundenkarten für ihr Handelsmarketing nutzen. Dabei wird es für das einzelne Handelsunternehmen zunehmend schwieriger, eine eigenständige Position im Kartenmarkt aufzubauen. Dies ist aber eine unabdingbare Voraussetzung für die Erreichung der ziele, die mit der Ausgabe von Kundenkarten verbunden sind.

Eine denkbare Weiterentwicklung der bestehenden Kartensysteme von Einzelhandelsunternehmen besteht in einer KonzernKarte (z.B. das Metro-Kaufhof-Massa-system). 42 Durch ein solches Kartensystem soll eine hohe Unternehmensgruppenbindung erzielt werden. Allerdings sind vor Einführung einer solchen Karte detaillierte Analysen hinsichtlich der Auswirkungen auf die Einzelunternehmen erforderlich, um deren Kundenbindung nicht $z u$ gefährden, negative unternehmensübergreifende Verbundwirkungen $z u$ erfassen und finanzielle Risiken zu begrenzen.

Alle Kartentypen können über die Zahlungsfunktion, die die Grundleistung der Karten darstellt, hinaus mit zusätzlichen Funktionen und serviceleistungen ausgestattet werden. Diese

42 Vgl. Schneider, M., Aufbruch in eine andere Welt, in: manager magazin, 11/1990, S. 316 ff. 
zusätzlichen Leistungen bieten das Potential für eine eigenständige Profilierung. Abbildung $7 \mathrm{gibt}$ in diesem Zusammenhang einen Überblick über unterschiedliche Ausstattungsmöglichkeiten von Kundenkarten. ${ }^{43}$

Dabei ist festzustellen, daß sich die angebotenen Zusatzleistungen vor allem auf einige relativ geringe warenbezogene Leistungen beschränken. Nur die Kartensysteme, bei denen die Konsumenten Beiträge leisten (z.B. Karstadt, IKEA, Metro und Tengelmann), bieten umfangreichere Zusatzleistungen an. Dies ist nur bedingt auf Kostenaspekte zurückzuführen. Vielmehr ist $z u$ berücksichtigen, daß das Wettbewerbsrecht keine kostenlosen Leistungen im Zusammenhang mit der Kundenkarte ge stattet. 44 Daher bewegen sich die zusatzleistungen gebührenfreier Kundenkartensysteme in relativ engen Grenzen.

Vgl. Zellekens, H.J., Fontaine, J., Braatz, F., Zahlung per Karte, a.a.o.; O.V., Kundenkarten im Einzelhandel, a.a.o.; O.V., Die Karten werden neu gemischt, a.a.o.; o.V., à la card, a.a.o.

$\mathrm{Zu}$ rechtlichen Problemen der Karte siehe $\$ 1$ Zugabeverordnung, \$2 Rabattgesetz, \$ 6 UWG. Vgl. Koch, H., Die große Lust am Kartenspiel, in: Manager Magazin, 11/1989, S. 287; o.V., Hertie gewinnt den Prozeß, in: Textilwirtschaft $\mathrm{Nr}$. 49, 12/1989, S. 23; 0.V., Kundenkarten im Einzelhandel, BTE Fachdokumentation, a.a.0., s. 3 ff. Der zulässige Barzahlungsrabatt beträgt $3 \%$. Darüber, ob dieser Rabatt Kundenkartenbesitzern gewährt werden darf, herrscht Rechtsunsicherheit. Verschärft wird dieses Problem bei Charge-Karten durch das zinsfreie Zahlungsziel. Vgl. v. Usslar, L., v. Morgen, R.D., Aktuelle Rechtsfragen der Kreditkartenpraxis, a.a.0., S. 108; v. Uzzlar, L., Anmerkungen zum Hertie-Urteil: Rechtssicherheit oder nicht?, in: à la card Aktuell, Nr. 1/1990, S. 18 f.; o.V., Komplettabdruck des Urteils der Dritten Kammer für Handelssachen am Landgericht Mannheim gegen die Goldene Kundenkarte von Hertie, in: à la card Aktuell, Nr. 3-Extra/1989, S. 1 ff. Zu Bestimmungen des Verbraucherkreditgesetzes vgl. v. Morgen, R.D., Neues Gesetz: Die Kreditkarte als Verbraucherkredit, in: à la card Aktuell, Nr. 22/1991, S. 7; auf das Gesetz bzgl. der allgemeinen Geschäftsbedingungen (AGBG) sowie das Gesetz bzgl. Abzahlungsgeschäfte $(\mathrm{AbzG})$ wird an dieser stelle nicht näher eingegangen, da sie eher die finanzrechtliche seite betreffen und auch in der Literatur kaum diskutiert wer-

$$
\text { Marcus Poggenpohl - 978-3-631-75099-5 }
$$




\begin{tabular}{|c|c|c|c|c|c|c|}
\hline \multirow{5}{*}{ 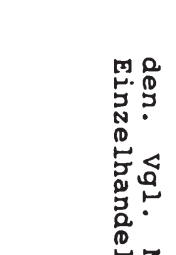 } & & Breuninger & Hertie & Karstadt & Kaufhof & Massa \\
\hline & Kartentyp & Kundenkarte & Kundenkarte & Kundenkarte & Kundenkante & Kundenkarte \\
\hline & Zlelgruppe & Stammkunden & Alle Kunden & Stammkunden & Stammkunden & Stammkunden \\
\hline & Voraussetzung & Bonttät/Nolljährigkelt & Bonität/Volljährigkelt & Bonität/Volljährigkelt & Bonität/Nolljährigkeit & Bonität/Volljährigkeit \\
\hline & Kosten & Keine & Keine & 50,- DM pro Jahr & Keine & 10,- DM pro Jahr \\
\hline \multirow{3}{*}{ 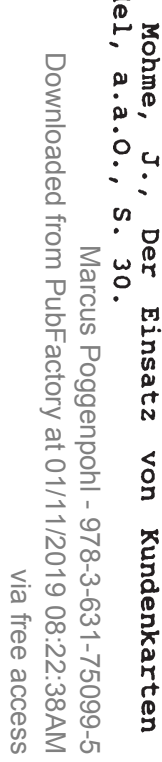 } & Abrechnung & $\begin{array}{l}\text { Monatlich per Last- } \\
\text { schrift oder Ūber- } \\
\text { welsung }\end{array}$ & $\begin{array}{l}\text { Per Kontoeinzug; } \\
\text { Abrechnung am 20. d. } \\
\text { Monats/Zahlung am } \\
\text { 10. des Folgemonats }\end{array}$ & $\begin{array}{l}\text { Monatl. Belastung am } \\
\text { 15. des Monats oder } \\
\text { 14-tägige Zahlungs- } \\
\text { frist bei Rechnung }\end{array}$ & $\begin{array}{l}\text { Monatl. Abrechnung; } \\
\text { Einzug per Lastschritt }\end{array}$ & $\begin{array}{l}\text { Abbuchung am 15. des } \\
\text { Folgemonats; Bel } \\
\text { Ratenzahlung } 4,2 \% \\
\text { effektiver Jahreszins }\end{array}$ \\
\hline & $\begin{array}{l}\text { Nutzer- } \\
\text { information }\end{array}$ & $\begin{array}{l}\text { o Veranstaltungs- } \\
\text { kalender }\end{array}$ & $\begin{array}{l}\text { o Monatl. Rechnungs- } \\
\text { bellagen } \\
\text { o Spezielle Mallings }\end{array}$ & $\begin{array}{l}\text { o Infobriefe zu Veran- } \\
\text { staltungen und } \\
\text { Sonderaktionen }\end{array}$ & $\begin{array}{l}\text { o Spezielle Mailings } \\
\text { o Prospekte als } \\
\text { Rechnungsbeilagen }\end{array}$ & $\begin{array}{l}\text { o Wöchentliche } \\
\text { Werbeprospekte }\end{array}$ \\
\hline & Leistungen & $\begin{array}{l}\text { o Bargeldloser Elnkauf } \\
\text { o Ware zur Ansicht } \\
\text { o Relsebuchungen im } \\
\text { Breuninger Relse- } \\
\text { burro } \\
\text { o Zinsloses Zahlungs- } \\
\text { zlel bis zu } 6 \text { Wochen } \\
\text { o Ratenzahlung } \\
\text { o Gutscheine für } \\
\text { Sonderlelstungen } \\
\text { o Kartenakzeptanz in } \\
60 \text { Pantnergeschăften }\end{array}$ & $\begin{array}{l}\text { o Bargeldloser Einkauf } \\
\text { o Zinsloses Zahlungs- } \\
\text { zlel bls zu } 6 \text { Wochen } \\
\text { o } 3000 \text {,- DM Kredit- } \\
\text { limtt pro Monat } \\
\text { o Bargeldlose Nutzung } \\
\text { aller Dienstleistungen } \\
\text { In den Filialen }\end{array}$ & $\begin{array}{l}\text { o Bargeldloser Einkauff } \\
\text { o Ware zur Ansicht } \\
\text { o Lleferung frel Haus } \\
\text { o Kostenloser Taxi-Ruf } \\
\text { o Geschenkver- } \\
\text { packungsservice } \\
\text { o Rückvergütung ab } \\
\text { 3000,- DM Ums.p.a. } \\
\text { o Paketaufbewahrung } \\
\text { o Kundengarderobe } \\
\text { o Bestellservice bel } \\
\text { Lebensmitteln } \\
\text { o Rückerstattung der } \\
\text { Parkgebühren } \\
\text { o Spezieller Kunden- } \\
\text { kartenbetreuer }\end{array}$ & $\begin{array}{l}\text { o Bargeldloser Einkauf } \\
\text { o } 3 \% \text { Rückvergütung } \\
\text { ab } 5000 \text {, - DM Kauf- } \\
\text { summe pro Jahr } \\
\text { o } 4000 \text {,- DM Kredit- } \\
\text { limit pro Monat } \\
\text { o Zinsloses Zahlungs- } \\
\text { ziel bls zu } 6 \text { Wochen } \\
\text { o Spezieller Kunden- } \\
\text { kartenbetreuer } \\
\text { o Geschenkver- } \\
\text { packungsservice } \\
\text { o Kostenloses Orts- } \\
\text { telephon } \\
\text { o 24-h Änderungs- } \\
\text { dienst }\end{array}$ & $\begin{array}{l}\text { o Bargeldloser Einkauf } \\
\text { o 2000,- DM Kredit- } \\
\text { limit pro Monat } \\
\text { o Reservierte Kassen } \\
\text { o Ratenzahlung } \\
\text { (slehe Abrechnung) }\end{array}$ \\
\hline
\end{tabular}

Abb. 7: Ausstattungsmerkmale ausgewählter Kundenkarten 


\section{Informationspotentiale von Rundenkarten für den Einzel- handel}

Häufig haben Unternehmen den Anspruch, daß sich eine Kundenkarte selbständig durch Gewinne finanziert. Dabei sind drei grundsätzliche Ansatzpunkte zu unterscheiden:

- Kosteneinsparung durch die Substitution von Kreditkartenumsätzen durch Kundenkartenumsätze. Die dadurch wegfallenden Disagiozahlungen bilden ein Ertragspotential, da die Kosten für die Abwicklung einer Kundenkarte unter den Disagiosätzen der Kreditkartenunternehmen liegen. 45

- Konsolidierung bestehender Umsätze und Generierung von Zusatzumsätzen.

- Eröffnung neuer Umsatzfelder im finanzwirtschaftlichen Bereich durch zinserträge aus der vergabe von Verbraucherkrediten. Dies kann allerdings nur erfolgen, wenn die Kundenkarte mit einer echten kreditfunktion ausgestattet ist.

Problematisch für eine erfolgreiche Kosten-/Ertragsanalyse der Kundenkarten ist, daß Kosteneinsparungen und zusatzumsätze nicht direkt nachgewiesen werden können. So könnte eine substitution nur dann bewiesen werden, wenn bei jedem Kundenkartenkauf gefragt würde, ob die Zahlung bei fehlender Kundenkarte mit einer kreditkarte getätigt worden wäre. Echte zusatzumsätze sind ebenfalls schwer zu erfassen, da es an Vergleichswerten fehlt, die nur durch Befragungen $z u$ erhalten sind. Insgesamt ist die Existenz und Höhe einer Ertragssteigerung durch Kundenkarten kaum nachweisbar. Zen-

45 Lt. Auskunft eines Handelsunternehmens liegen die Kosten für die Abwicklung einer Kundenkarte bei ca. 1,2\% vom Umsatz. 
trale nachweisbare Potentiale der Kundenkarte liegen jedoch im Bereich der Informationsgewinnung.

Bei zunehmender Wettbewerbsintensität im Handel sind Informationen über den Kunden und sein Kaufverhalten von zentraler Bedeutung. In diesem Zusammenhang stellen Kundenkartendaten ein umfangreiches Informationspotential dar. Dabei sind die objektiven Daten über das Kaufverhalten von besonderer Bedeutung. Bisher war es nur möglich, auf Untersuchungen der BAG (Bundesarbeitsgemeinschaft des Groß- und Einzelhandels) zurückzugreifen. Eine Aktualität der Daten ist in diesem Zusammenhang kaum gegeben, da die Untersuchung nur in einem Vierjahres-Rhythmus durchgeführt wird. Darüber hinaus werden für diese Untersuchungen nur drei Tage als Testzeitraum angesetzt. Ebenso sind die Ergebnisse unterschiedlicher Panelerhebungen häufig wenig aufschlußreich, da bei diesen der Bezug zu den spezifischen Entscheidungssituationen des jeweiligen Unternehmens fehlt.

Als Alternative $z u$ sekundärstatistischen Analysen kann der Handel eigenständige Untersuchungen durchführen. Neben dem hohen Kostenaufwand wird auch dabei nur ein begrenzter Zeitausschnitt analysiert. Zudem werden bei diesen Untersuchungen demographische Daten nur bedingt erfaßt.

Der Einsatz der Kundenkartendaten stellt einen Ausweg aus diesem "Informationsdilemma" im Handel dar. Die bei der Kundenkarte anfallenden Informationen können den Bedarf nach aktuellen Informationen $z . T$. befriedigen. Die Daten 46

- sind tagesaktuell verfügbar,

- mit demographischen Angaben zu koppeln,

- spiegeln tatsächliche Kaufvorgänge wider und strument der Verbundanalyse im Einzelhandel, a.a.0., s. 25. 
- fallen bei der Benutzung einer Karte automatisch und somit kostenlos an. 47

Die Aussagefähigkeit für das Handelsmarketing ist in besonderem Maße von der Hard- und Software, die zur Abwicklung der Karte eingesetzt wird, abhängig. Denn diese hat starke Auswirkungen auf Aktualität und Umfang der Informationen, die gewonnen werden. Darüber hinaus ist die struktur und Art der gewonnenen Informationen von zentraler Bedeutung.

Neben kaufbezogenen Größen ist über eine Kontonummer datentechnisch eine Verbindung $z u$ den demographischen Daten des Kunden möglich. Im Falle eines filialisierten Unternehmens ist zudem eine Identifizierung der jeweiligen Filiale notwendig. Wenn das Unternehmen davon ausgeht, daß die Umsätze eines Kunden stets in der gleichen Filiale getätigt werden, so ist diese Identifizierung auch über die Kontonummer zu erreichen.

Aufgrund dieser Voraussetzungen ist festzustellen, daß insgesamt alle Auswertungen, die z.B. auf Basis von Scannerdaten durchgeführt werden, 48 auch mit Kundenkartendaten durchführbar sind. Darüber hinaus besteht auch die Möglichkeit einer integrierten Analyse des Kaufverhaltens und der Demographie.

47 Die Kostenneutralität bezieht sich nur auf die Datenerhebung. Speicherung, Transfer und Weiterverarbeitung stellen einen Aufwand dar.

48 Zur Nutzung von Scannerdaten vgl. z.B. Heidel, B., Die Nutzung von Scannerdaten zur Planung der Absatzpolitik in Einzelhandelsbetrieben, Diss., Trier 1989; Simon, H., Entscheidungsunterstützung mit Scannerdaten, in: Simon, H. (Hrsg.), Marketing im technologischen Umbruch, Stuttgart 1987; Simon, H., Kucher, E. Sebastian, K.H., Scanner-Daten in Marktforschung und Marktentscheidung, in: ZFB 1982, S. 555-597; Kucher, E., Höhere Marketing Effizienz mit Scanner-Daten, in: asw, 2/1983, S. 83-89. 
Nicht zuletzt ist die Vorbereitung der direkten und kaufverhaltensspezifischen Kundenansprache innerhalb des Direktmarketing Aufgabe der Kundenkarte. Für die Qualität des Direktmarketing sind die Informationsgrundlagen von besonderer Bedeutung. Eine weitere zentrale Aufgabe der Kundenkarte für das Handelsunternehmen ist daher die Beschaffung von qualifizierten Adressen neben den Informationen bzgl. des Kaufverhaltens, die die Bedürfnisse des Kunden widerspiegeln.49

Die Bedeutung für das Controlling geht bereits aus den Voraussetzungen für die Ausgabe einer Kundenkarte hervor. Es besteht ein system, das in der Lage ist, ständig aktuelle und kundenbezogene Informationen zu liefern. 50 Diese beziehen sich nicht nur auf das Unternehmen als ganzes sondern sie können auf einzelne Geschäftsstätten oder Abteilungen bezogen werden.

Dabei ist nicht allein die Messung des Abverkaufs von beworbenen Produkten oder der Entwicklung einer Abteilung möglich. Es kann vielmehr untersucht werden, wie sich die Kunden innerhalb der Geschäftsstätte bewegt haben, sofern sie unterschiedliche Kassen benutzen. Dadurch sind Kundenlaufstudien zwar aufgrund fehlender zusatzinformationen (z.B. Suchverhalten etc.) nicht zu ersetzen, doch sind kostenneutral auch räumliche Kaufvorgangsfolgen $z u$ erheben. Nur die zeit vor dem ersten und nach dem letzten getätigten Kauf ist nicht kontrollierbar. 51

49 Vgl. Kreuzer, R.T., Weppler, K., Marketing by Database Eine Herausforderung für Markenartikler, a.a.o., S. 498 f.

50 Vgl. auch Mohme, J., Der Einsatz von Kundenkarten im Einzelhandel, a.a.0., s. $6 \mathrm{f}$.

51 Vgl. Poggenpohl, M., Kundenkarteninformationen als Instrument der Verbundanalyse im Einzelhandel, a.a.o., s. $57 \mathrm{f} .$, auch $z u$ den nicht kontrollierbaren störgrößen bzgl. der Erfassung der Zeiträume zwischen Käufen wie Marcus Poggenpohl - 978-3-631-75099-5 
Über diese Aspekte hinaus kann sowohl im Rahmen einzelner Aktionen als auch aktionsübergreifend festgestellt werden, was ein bestimmter Kunde innerhalb eines Kaufaktes bzw. eines zeitraums gekauft hat. Durch dieses Vorgehen werden Verbundanalysen ermöglicht. Diese Analysen setzen verschiedene Käufe miteinander in Beziehung und sind somit in der Lage, hochkomplexe Kaufverbundvorgänge abzubilden und zu analysieren. Somit liegt hier ein wesentliches Informationspotential von Kundenkarten.

Kundenkarteninformationen bieten Möglichkeiten zur Verbesserung der Qualität der Informationsauswertung im Bereich der Verbundforschung. Dies ist zum einen auf die kontinuierliche Erfassung umfangreicher Kaufdaten zurückzuführen, die es ermöglicht, durchgeführte Untersuchungen bei relativ geringem Aufwand $z u$ wiederholen und damit die Ergebnisse zu kontrollieren und $\mathrm{zu}$ verbessern. Zum anderen können durch die Hinzunahme demographischer Daten Erkenntnisse über das Kaufverhalten unterschiedlicher Gruppen gewonnen werden. Dadurch wird es dem Handelsunternehmen ermöglicht, Verbundbeziehungen innerhalb unterschiedlicher zielgruppen festzustellen sowie deren Verhaltensweisen und Kaufstrukturen miteinander $z u$ vergleichen. Dies stellt die Basis für eine spezifische Anpassung von Maßnahmen auf bestimmte Marktsegmente dar. Auch hier bietet sich durch regelmäßige wiederholung die Möglichkeit, Verhaltensänderungen innerhalb von zielgruppen festzustellen.

Die Nutzung von Kundenkarteninformationen bedeutet eine qualitative Verbesserung der Kaufverbundanalyse als Zeitpunktbetrachtung. Besondere Bedeutung haben jedoch Auswertungen von zeitraumbezogenen verbundbeziehungen, die durch die Nutzung der Kundenkartendaten ermöglicht werden. Vor allem nä-

z.B. Beratungsgespräche, Einkaufpausen, orientierungsprobleme etc. 
hern sich diese Analysen wesentlich stärker den tatsächlichen Bedarfsstrukturen der Kunden an, die der Kaufverbund nur sehr unvollständig abbilden kann. Mit Hilfe der Kundenkarteninformationen läßt sich der Bedarfsverbund der Nachfrager erheblich besser abbilden als mittels der Kaufverbundanalyse auf Basis von Kassenbons.

Insgesamt führt die Nutzung von Kundenkartendaten $z u$ einer optimierung und Ausweitung der Verbundanalysen. Dadurch verbessert sich auch die Qualität der Ergebnisse dieser Analysen als Grundlage für Entscheidungsprobleme des Handels. 52

\section{Zielsetzung und Gang der Untersuchung}

Zielsetzung der Untersuchung ist es, einen Beitrag zur wissenschaftlichen weiterentwicklung des verbundkonstruktes zu leisten. Darüber hinaus werden Hinweise für die Praxis gegeben, wie eine Nachfrage- und Kaufverbundanalyse auf Basis von Kundenkarteninformationen durchzuführen ist, welche Informationen $\mathrm{zu}$ gewinnen sind und für welche Entscheidungen sie genutzt werden können. Auf diese Weise wird die Leistungsfähigkeit des Instrumentes Verbundanalyse auf der Grundlage von Kundenkarteninformationen herausgearbeitet.

Die wissenschaftliche zielsetzung untergliedert sich zum einen in eine theoretisch-begriffliche und zum anderen eine methodische Zwecksetzung. Das theoretisch-begriffliche ziel bezieht sich auf die Erweiterung des Verbundbegriffes durch die Einbeziehung eines zeitraumaspektes. Der methodische Teil bezieht sich auf die Untersuchung der bisher angewandten Methoden und die Entwicklung einer Vorgehensweise zur Erfassung des Nachfrageverbundes als zeitraumbezogenes Kon- 
strukt. Darüber hinaus wird eine Marktsegmentierung auf Basis der Ergebnisse der Nachfrageverbundanalyse durchgeführt und auf ihre Eignung zur Bildung von zielgruppen überprüft.

In Kapitel B. steht zunächst die Erweiterung des Verbundkonstruktes von einem rein zeitpunktbezogenen Konzept auf ein zeitraumbezogenes Konzept im Vordergrund. Damit werden die Grundlagen für die weitere Diskussion des Verbundkonstruktes gelegt. Aufbauend auf einer Darstellung der Verbundmessung in der bisherigen Forschung, die sich sowohl auf die Darstellung und Analyse klassischer Verbundkonstrukte als auch auf die empirische Verbundforschung erstreckt, erfolgt eine Diskussion der bei der empirischen Forschung einzusetzenden Analyseverfahren. Daraus wird dann das methodische Vorgehen der empirischen Untersuchung abgeleitet. Eine ausführliche Diskussion der Einflußgrößen des Kauf- und Nachfrageverbundes stellt die Grundlage zur Ableitung von Hypothesen bzgl. der Wirkung von Einflußgrößen dar. Die Operationalisierung des Verbundbegriffes und die Untersuchung der Besonderheiten der Verbundmessung anhand von Kundenkarten schließen diesen Teil der vorliegenden Untersuchung ab.

Im Teil C werden die präzisierten Verbundkonstrukte, aufbauend auf einer detaillierten Darstellung des Untersuchungsdesigns und der Kontextdimensionen der analysierten Unternehmung empirisch überprüft. Die allgemein hergeleiteten Vorgehensweisen der Verbundmessung werden im Rahmen der empirischen Untersuchung auf dem Aggregationsniveau der Beziehung zwischen Abteilungen angewendet. Dabei werden zunächst der zeitpunktbezogene und anschließend auch der zeitraumbezogene Verbund gemessen und interpretiert. Beim Nachfrageverbund werden dabei auch demographisch determinierte Untergruppen analysiert und die jeweiligen Ergebnisse vergleichend auf Konvergenzen bzw. Divergenzen untersucht. 
Aufbauend auf diesen Ergebnissen wird überprüft, ob sich die Verbundmessung auch als Grundlage zur Zielgruppenbildung eignet, und analysiert, wie die gebildeten Gruppen gekennzeichnet werden können. Eine Ableitung von Implikationen für das Management im Einzelhandel schließt diesen Teil ab.

In der Schlußbetrachtung im Teil D erfolgt eine zusammenfassende Darstellung der Untersuchungsergebnisse sowie eine Diskussion der Ansatzpunkte für die weiterführende Forschung. 


\section{B. Theoretische Grundlagen der Verbundmessung im Handel}

\section{Ansätze zur Verbundmessung im Handel}

\subsection{Das system unterschiedlicher Verbundarten}

In der betriebswirtschaftlichen Literatur zum Käuferverhalten und zum Handelsmanagement finden sich eine Fülle von Begriffen und Konstrukten, die sich auf den sortimentsverbund beziehen. Dabei werden unterschiedliche Sachverhalte z.T. mit dem gleichen Begriff belegt. 1

Ausgangspunkt dieser Untersuchung bildet der Sortimentsverbund als oberbegriff. ${ }^{2}$ Er kennzeichnet alle, sowohl von der Angebots- als auch von der Nachfragerseite ausgelöste Ausstrahlungseffekte innerhalb eines sortiments. Daraus läßt sich eine sinnvolle Unterteilung des Sortimentsverbundes in einen nachfrage- und einen angebotsinduzierten Verbund ableiten. ${ }^{3}$ Im weiteren Verlauf wird nur noch der nachfrageinduzierte Verbund betrachtet.

Vgl. Merkle, E., Die Erfassung und Nutzung von Informationen über den Sortimentsverbund in Handelsbetrieben, in: Schriften zum Marketing, Dichtl, E., Böcker, F. (Hrsg.), Bd. 11, Berlin 1981, S. 2 .

2 Vgl. Böcker, F., Die Bestimmung der Kaufverbundenheit von Produkten, in: Schriften zum Marketing, Dichtl, E., Böcker, F. (Hrsg.), Bd. 7, Berlin 1978, S. 20; Merkle hingegen ordnet den Sortimentsverbund hierarchisch tiefer ein; vgl. dazu Merkle, E., Die Erfassung und Nutzung von Informationen über den Sortimentsverbund in Handelsbetrieben, a.a.0., S. 5 .

3 Böcker definiert den konsumenteninduzierten Verbund als Nachfrageverbund und erfaßt auf einer untergeordneten stufe noch eine Nachfrageverbundenheit, die er auf die gleiche stufe wie eine Bedarfsverbundenheit einordnet; vgl. Böcker, F., Die Bestimmung der Kaufverbundenheit von Produkten, a.a.O., S. 20; zu entsprechendem Vorgehen vgl. Bass, F.M., Pessemier, E.A., Tigert, D.J., Complementary and substitute patterns of purchasing and use, in: JAR, 2/1969, S. 19; Engelhardt, W.H., Erscheinungs- 
Damit sind im weiteren die unterschiedlichen Verbundarten eines Individuums oder einer fest definierten Gruppe von Individuen (z.B. einem Haushalt) von Interesse. Die Basis der Verbundhierarchie ist dabei der Bedarfsverbund:

Der Bedarfsverbund spiegelt die Menge aller von einem Individuum oder einem Haushalt in einem bestimmten Zeitraum benötigten Güter wider. ${ }^{4}$

Darauf aufbauend ergibt sich ein vierstufiges Hierarchiesystem von Verbundbegriffen, die allesamt zum konsumenteninduzierten Verbundsystem zählen. Einen Überblick über die unterschiedlichen Begriffe gibt Abbildung 8.5

In der Verbundhierarchie folgt dem Bedarfsverbund der Nachfrageverbund. 6

Der Nachfrageverbund umfaßt alle von einem Individuum oder einem Haushalt tatsächlich nachgefragten Güter innerhalb eines Zeitraumes.

formen und absatzpolitische Probleme von Angebots- und Nachfrageverbunden, in: $\mathrm{ZfbF} 1976$, S. $77 \mathrm{f}$.

4 Einer eher durch den Verwendungszweck bestimmten Festlegung des Bedarfsverbundes wird hier nicht gefolgt. Vgl. Heinemann, G., Betriebstypenprofilierung im Facheinzelhandel - Ansätze der strategischen Marktsegmentierung, Arbeitspapier Nr. 35 des Intituts für Marketing der Universität Münster, Meffert, H. (Hrsg.), Münster 1987, S. 33; Medla, K., Shop-in-the-shop, ein Konzept der Angebotspräsentation, München 1987 , S. 49.

5 Da nur der unternehmensspezifische Nachfrageverbund empirisch klar zu erfassen ist, ist immer dieser gemeint, wenn im weiteren Verlauf der Untersuchung vom Nachfrageverbund die Rede ist.

6 Riebel verwendet die Begriffe Bedarfs- und Nachfrageverbundenheit als synonyme, ohne die möglichen Differenzen zwischen den beiden Begriffen aufzugreifen. Vgl. Riebel, P., Kosten und Preise bei verbundener Produktion, Substitutionskonkurrenz und verbundener Nachfrage, opladen 1971 , S. 48 . 


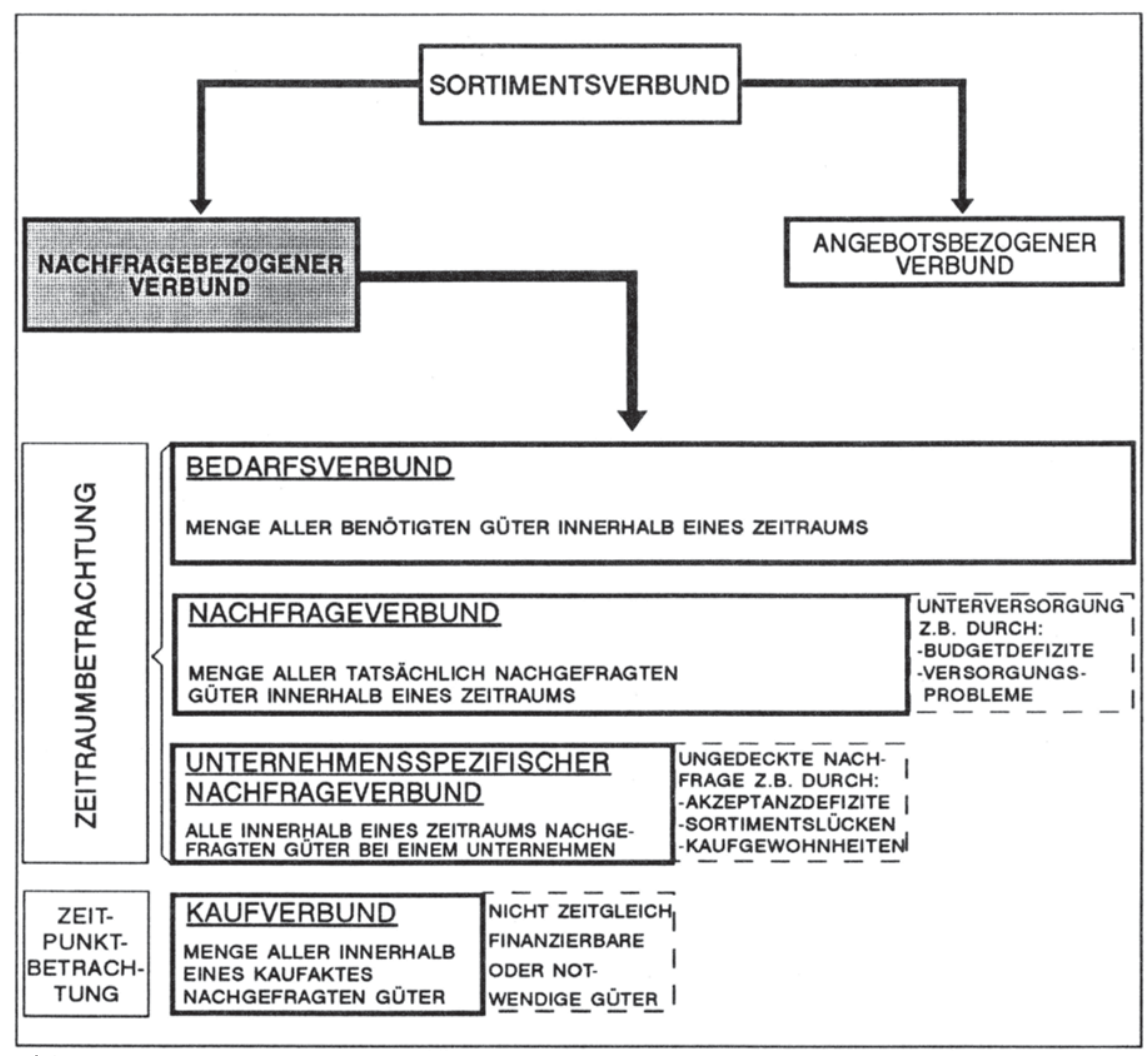

Abb. 8: Definition und Hierarchie unterschiedlicher Verbundbeqriffe

Sind die Zeiträume bei Bedarfs- und Nachfrageverbund gleich definiert, so sind im Idealfall Bedarfs- und Nachfrageverbund deckungsgleich. Es kann jedoch auch zu einer Divergenz zwischen den beiden Verbundarten kommen. Dies kann zum einen durch Budgetdefizite auf der Nachfragerseite ausgelöst sein, sofern das Budget nicht für alle benötigten waren oder Dienstleistungen ausreicht. Zum anderen kann die Divergenz aber auch durch Beschaffungsbarrieren ausgelöst werden. Darunter fallen sowohl die mangelnde Ubiquität benötigter waren, z.B. ausgelöst durch schwächen in der Infrastruktur eines Handelsbetriebes, oder auch das generelle Fehlen benötigter waren aufgrund echter Beschaffungsengpässe. Beides

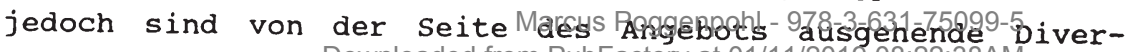
Downloaded from PubFactory at 01/11/2019 08:22:38AM 
genzen, so daß sich auch in der struktur des Verbundes auf der Nachfragerseite angebotsbedingte Einflüsse zeigen können.

Die unterste stufe der Verbundhierarchie bildet der Raufverbund (vgl. Abbildung 8 ):

Der Kaufverbund ist die Menge aller von einem Individuum oder einem Haushalt $z u$ einem Zeitpunkt nachgefragten Güter. Da der Kaufverbund sich auf einen Zeitpunkt bezieht, ist es sinnvoll, ihn auf die Menge aller in einem Unternehmen gekauften Güter $z u$ beziehen.

Für eine analytische Erfassung der unterschiedlichen Verbundarten ist es notwendig, alle Käufe eines Konsumenten zu erfassen. Dies geschieht in den bisherigen Untersuchungen mit Hilfe von Warenkorbbeobachtungen bzw. Kassenbonprüfungen. ${ }^{7}$ Beide Methoden erfordern jedoch aus Gründen der praktischen Durchführbarkeit die Beschränkung der Erfassung auf eine einzelne Geschäftsstätte. Daraus ergibt sich noch keine Auswirkung für die Erfassung des Kaufverbundes. Betrachtet man jedoch die Ebene des Nachfrageverbundes, so ist festzustellen, daß keine automatische Einengung auf eine Geschäftsstätte durch die Definition des Nachfrageverbundes gefordert wird. Um eine definitorisch einwandfreie Grundlage für die weiteren Ausführungen $z u$ erhalten, ist es daher notwendig, einen weiteren Begriff einzuführen, den Begriff des unternehmensspezifischen Nachfrageverbundes:

7 Vgl. z.B. Böcker, F., Die Bestimmung der Kaufverbundenheit von Produkten, a.a.o.; Stahl, P., Verbundwirkungen im Sortiment - Ein Beitrag zur Erfassung und Messung von Verbundwirkungen im Sortiment von Handelsbetrieben, Diss., Münster 1977; Fischer, T., Die Warenkorbanalyse als Instrument zur experimentellen Verbesserung des Einsatzes absatzseitiger Aktionsparameter im Lebensmitteleinzelhandel - Theorie, Methodik und empirische Analyse, Diskussionspapier des Lehrstuhls für Betriebswirtschaftslehre, insbesondere Distribution und Handel der Universität Münster, Münster 1991. 
Unter einem unternehmensspezifischen Nachfrageverbund versteht man die Menge aller von einem Individuum oder einem Haushalt bei einem Unternehmen nachgefragten Güter innerhalb eines bestimmten Zeitraumes.

Dabei können die Divergenzen zwischen dem allgemeinen Nachfrageverbund und dem unternehmensspezifischen Nachfrageverbund z.B. durch Akzeptanzprobleme bestimmter sortimentsbereiche beim untersuchten Unternehmen, durch tatsächlich bestehende Sortimentslücken, durch bestehende Kaufgewohnhei$\operatorname{ten}^{8}$, die Einkaufsstättenwahl ${ }^{9}$ oder durch zufallsbedingte Einflüsse ausgelöst werden. Es ist jedoch eindeutig, daß diese Divergenz kleiner wird, je umfassender das Sortiment des Unternehmens angelegt ist. Im idealen Fall ist das Unternehmen ein Vollsortimenter, der weitestgehend den Bedarf der betrachteten Haushalte deckt. Bei dieser sehr breiten sortimentsabdeckung wird dem Konsumenten zumindest die Chance gegeben, jedes benötigte Gut in dem betrachteten Handelsunternehmen $z u$ kaufen. Damit besteht die Möglichkeit, einen Großteil der Verbundstruktur eines Individuums oder Haushaltes aufzudecken. Die sortimentslücken und darüber hinaus die bestehenden Divergenzen, die durch Akzeptanzprobleme oder Kaufgewohnheiten ausgelöst werden, sind nicht Gegenstand dieser Untersuchung. Allerdings wird die Akzeptanzproblematik bei der späteren Interpretation von Verbundbeziehungen in die Diskussion einbezogen.

Zum Bereich Kaufgewohnheit vgl. z.B. Dieterich, M., Konsument und Gewohnheit - eine theoretische und empirische Untersuchung zum habituellen Kaufverhalten, Heidelberg 1986; Kaas. R., Dietrich, M., Die Entstehung von Kaufgewohnheiten bei Konsumenten, in: Marketing, Heft 1/1979, S. $13 \mathrm{ff}$.

9 Zur Einkaufsstättenwahl vgl. z.B. Heinemann, M., Einkaufsstättenwahl und Firmentreue, Münster 1974 ; Weinberg, P., Das Entscheidungsverhalten der Konsumenten, Paderborn 1981 . 


\subsection{Forschungsansätze zur Verbundanalyse}

\subsection{Klassische Ansätze zur Verbundmessung}

In der betriebswirtschaftlichen Forschung wurde das Thema "Verbundmessung" vor allem in den 60er Jahren bis zum Beginn der soer Jahre behandelt. Erste mikroökonomische Ansätze, die sich mit Verbundeffekten befaßten, um einen Beitrag zur Verbesserung der Preispolitik in Mehrproduktunternehmen $z u$ leisten, wurden bereits in den 50er Jahren entwickelt. Dabei standen in bezug auf den Handel Ausgleichskalkulationen und die zurechnung von Gewinnanteilen auf einzelne Bestandteile des Sortiments bzw. Produktprogramms im Mittelpunkt der Diskussion. 10 Eine umfassende Erforschung eines Sortimentsverbundes erfolgte dabei nicht. In der Mehrzahl der Veröffentlichungen wird allenfalls auf die Existenz von Verbundbeziehungen und deren Einflüsse verwiesen. 11

Auch die warenorientierte Absatzwirtschaftslehre hat sich zumindestens indirekt - mit der Verbundanalyse im Handel auseinandergesetzt. 12 so wird beim "commodity approach" aufgezeigt, nach welchen Kriterien Sortimentsgruppen beschrie-

10 Vgl. Horacek, M., Der kalkulatorische Ausgleich, Wien 1950; Humbel, P., Preispolitische Gewinndifferenzierung im Einzelhandel, Zürich 1958; Albach, A., Zur Sortimentskalkulation im Einzelhandel, in: Handelsbetrieb und Marktordnung, Festschrift, Wiesbaden 1962, S. 13-40; Schmitz, G., Kalkulatorischer Ausgleich als betriebspolitische Aufgabe, in: Distributionswirtschaft, Sundhoff, E. (Hrsg.), Köln, Opladen 1968, S. 1-27; Ott, A.E., Grundzüge der Preistheorie, Göttingen 1968.

11 Vgl. Fry. J.N., Family branding and consumer brand choice, in: JMR 1967, S. 237-247; Knoblich, H., Betriebswirtschaftliche Warentypologie, Grundlagen und Anwendungen, Köln, Opladen 1969.

12 Vgl. Böcker, F., Die Bestimmung der Kaufverbundenheit von Produkten, a.a.0., S. 39. 
ben werden können. ${ }^{13}$ Dies impliziert eine Beschreibung von Produktgruppen anhand von Ähnlichkeitsmerkmalen. Die Ergebnisse der Überlegungen beschränken sich weitgehend auf beschreibende Ausführungen zur Typenbildung. Die in diesen Beschreibungen enthaltenen Kriterien bieten aufgrund von Unschärfen und mangelnder operationalisierbarkeit für eine empirische Verbundforschung keine ausreichende Fundierung. 14 Aber auch konzeptionell stellt die Ähnlichkeit von Produkten keine für die Erforschung des Kaufverbundes ergiebige Basis dar, da der Kauf- und auch der Nachfrageverbund nur von der Konsumenten- und nicht von der Produktseite her zu erfassen ist und deshalb Produktähnlichkeiten eine untergeordnete Bedeutung zukommt.

Im Bereich der Mikroökonomie sind mehrere Ansatzpunkte der Verbundanalyse $z u$ unterscheiden, die jedoch nicht vollständig unabhängig voneinander sind. In der Preis- und Nutzentheorie wird eine Verbundenheit anhand der Größen Substitutionalität und Komplementarität gemessen. Diese Größen können sowohl technisch als auch verhaltensbedingt erklärt werden. Es wird zwischen variablem und fixem Einsatzverhältnis bei Verbundbeziehungen unterschieden. ${ }^{15}$ Da der Kauf- und Nachfrageverbund aber auch Güter betrifft, die in keinerlei substitutionalem Verhältnis stehen, also nach dieser Betrachtungsweise nicht verbunden sind, führt auch dieser Ansatz konzeptionell kaum weiter. 16

13 Vgl. Knoblich, H., Warentypologie, in: Tietz, B. (Hrsg.), Handwörterbuch der Absatzwirtschaft, stuttgart 1974 , Sp. 2175 ff.

14 Vgl. Böcker, F., Die Bestimmung der Kaufverbundenheit von Produkten, a.a.O., S. 40 .

15 Vgl. Ott, A.E., Grundzüge der Preistheorie, Göttingen 1968, S. 105-107.

16 Geringe oder fehlende Verbundenheit wird in diesen Ansätzen mit dem Konzept einer Substitutionslücke erklärt. Vgl. Robinson, J., The economics of imperfect competiMarcus Poggenpohl - 978-3-631-75099-5 
Aus der Grenzrate der Substitution kann eine operationalisierung der nutzentheoretischen Verbundenheitsauffassung abgeleitet werden. Unter der Grenzrate der substitution ist die Menge eines Gutes zu verstehen, die im Urteil eines Befragten den Wegfall einer Einheit eines anderen Gutes kompensiert. 17 Auch diesem Ansatz liegt die Vorstellung zugrunde, daß jedes Gut "mehr oder weniger ein Komplement zu jedem anderen ist". 18 Ermittelt wird die Grenzrate der substitution gewöhnlich mit Hilfe von Elastizitätskoeffizienten. 19

Werden diese Koeffizienten als Verbundmaß herangezogen, so drückt ein hoher positiver wert auch hohe substitutionalität aus. Problematisch ist dabei, daß unter der Annahme der Substitutionalität des untersuchten Güterpaares keine Komplementarität $z u$ erzielen ist und somit Verbundwirkungen per definitione entfallen. Gemessen wird dabei lediglich die Substitutionalität zweier Güter in bezug auf ein Budget. Somit erscheint auch dieser Ansatz als Grundlage für Verbundanalysen im Handel wenig geeignet.

Die Messung von Verbundenheit mit Hilfe von Kreuzpreiselastizitäten erscheint ebenfalls problematisch. Auch hier wird ein Komplementaritäts- bzw. Substitutionalitätsmaß herangezogen. Aus einer positiven Kreuzpreiselastizität wird der Schluß gezogen, daß die beiden betrachteten Güter substitu-

tion, London, New York 1961; Böcker, F., Die Bestimmung der Kaufverbundenheit von Produkten, a.a.O., S. 56 f.; ott, A.E., Grundzüge der Preistheorie, a.a.0., S. $45 \mathrm{ff}$.

17 Vgl. Böcker, F., Die Bestimmung der Kaufverbundenheit von Produkten, a.a.0., S. 60 .

18 Krelle, W., Präferenz- und Entscheidungstheorie, Tübingen 1968 , S. 17 .

19 Vgl. Böcker, F., Die Bestimmung der Kaufverbundenheit von Produkten, a.a.0., S. 62 . 
tionsgüter sind. 20 Der Kernpunkt der Kritik an diesem Ansatz ist in der Tatsache zu sehen, daß sich nur Verbundwirkungen feststellen lassen, wenn der Anbieter eine Veränderung $z . B$. in seiner Preissetzung durchführt. Bei konstantem absatzpolitischem Umfeld sind keine Verbundgrößen erfaßbar.

Auch auf dem Gebiet der Operations Research-Forschung wurden Ansätze zur Erfassung von Verbundeffekten entwickelt. Dabei erfolgt eine Anpassung der theoretischen Ansätze und deren Informationsgrundlagen an die Möglichkeiten der Informationsbeschaffung. Ziel dieser Analysen war die Ermittlung optimaler Warengruppenstrukturen von Einzelhandelsunternehmen unter innerbetrieblichen und marktbezogenen Restriktionen. 21 Vor allem diese restriktiven Bedingungen, die zu unrealistischen Modellen führen, lassen die Ansätze letztendlich zur Erfassung empirischer Verbundeffekte nicht geeignet erscheinen. 22

Gegenüber diesen eher mathematisch-quantitativ orientierten Ansätzen befaßte sich schließlich eine Forschungsrichtung auch mit der qualitativen Diskussion des Verbundes. Dabei sind vor allem Beiträge zur Entwicklung von Strategien bzgl. des Einsatzes der Marketinginstrumente im Rahmen der Sor-

20 Vgl. Kotler, Ph., Marketing decision making: A model building approach, New York 1971, S. 164.

21 Vgl. Brauer, K.M., Die Ermittlung der optimalen Warengruppenstruktur einer Filiale in Lebensmittelfilialbetrieben, in: Unternehmensforschung im Handel, Gümbel, $R$. et al. (Hrsg.), Rüschlikon Zürich 1969, S. 53-124; Rühli, E., Optimale Sortimentsplanung mit linearer Programmierung, Zürich 1963.

22 Vgl. zur Eignung von Programmieransätzen der linearen Programmierung bei Kosten- und Absatzverbund Liedtke, P., Absatz- und Kostenverbundenheiten handelsbetrieblicher Leistungskomponenten und Leistungen und ihre Bedeutung für die sortimentspolitik der Handelsbetriebe, Diss., Gießen 1969; die Erfassung von Verbundwirkungen mit OR-Modellen ist vor dem Hintergrund der Unabhängigkeit der Variablen grundsätzlich problematisch. 
timentspolitik entstanden.23 Das eigentliche Problem der Messung des Verbundes wird dabei jedoch ausgeklammert, so daß diese Ansätze zur empirischen Forschung nicht beitragen können. Auch die Erfassung des Verbundes über Indikatoren brachte letztlich keine Lösung. 24

\subsection{Empirische Ronzepte zur Erfassung des Verbundes im Einzelhandel}

\subsection{Empirische Untersuchungen im Überblick}

In den 70er Jahren wurde durch die Einführung von Datenkassen die Informationsbeschaffung als Grundlage der Verbundanalyse vereinfacht. Zudem wurden in der statistik zunehmend auch multivariate Verfahren eingesetzt. Die Analyse von Warenkörben bzw. Kassenbons ermöglichte erstmals eine umfassende Erfassung von Beziehungen im Sortiment. 25 Durch diese

23 Vgl. zu Problemen des sortiments und der Verbundbeziehungen zwischen Sortimentsteilen Huwyler, M., Sortiment und Kaufhandlung, Diss. Bern, Zürich 1966; Bartels, G., Diversifizierung - die gezielte Ausweitung des Leistungsprogramms der Unternehmung, Stuttgart 1966; zu Interdependenzen $z$ wischen allen Marketinginstrumenten: Aspinwall, L., Characteristics of Goods Theory, in: Managerial Marketing, Lazer, W., Kelley, J. (Hrsg.), Homewood Ill. 1962, S. 633 ff.; Lipson, H.A., Darling, J.R., Reynolds, F.D., A Two-Phase Interaction Process for Marketing Model Construction, in: MSU Business Topics, Autumn 1970, S. $34 \mathrm{ff}$.

24 Vgl. Brede, H., Möglichkeiten zur Bestimmung der Nachfrageverwandtschaft mit Hilfe von Indikatoren, Habilitationsschrift, Frankfurt 1974; Brede, H., Absatzpolitik mit Hilfe der Nachfrageverwandtschaft, Wiesbaden 1976.

Vgl. Böcker, F., Die Analyse des Sortimentsverbundes Eine empirische Untersuchung, in: Perspektiven des Marketing im Handel, Blümle, E.B., Ulrich, W. (Hrsg.), Freiburg 1974, S. 55-81; Stahl, P., Verbundwirkungen im Sortiment - Ein Beitrag zur Erfassung und Messung von Verbundwirkungen im Sortiment von Handelsbetrieben, Diss. Münster 1977; Böcker, F., Merkle, E., Mantel Marcus Poggenpohl - 978-3-631-75099-5 
Analysen soll der Kaufverbund abgebildet werden. Dabei werden unterschiedliche mathematische Koeffizienten bzgl. ihrer Eignung zur Verbundmessung analysiert. ${ }^{26}$ Grundsätzlich sind zur Analyse von Verbundeffekten Korrelations- und Regressionsverfahren geeignet. Eine Weiterverarbeitung von Korrelationstabellen mit Hilfe der multidimensionalen skalierung (MDS) oder der Clusteranalyse führt zu aussagefähigen Ergebnissen für unterschiedliche Zwecke. 27

Vor allem in den 80er Jahren wurde die Verbundmessung schließlich zur Analyse von Spezialproblemen herangezogen. $\mathrm{Zu}$ diesen Problembereichen zählen beispielsweise:

"kauft" Bluse - Analyse des Sortimentsverbundes, in: Rationeller Handel, 10. Jg., Heft 1, 1975, S. 14-20; Böcker, F., Die Analyse des Kaufverbundes - ein Ansatz zur bedarfsorientierten Warentypologie, in: ZfbF, 27 . Jg., Heft 5, 1975, S. 290-306; Böcker, F., Merkle, E., Die Analyse des Sortimentsverbundes, in: Erfolgskontrolle im Marketing, Böcker, F., Dichtl, E. (Hrsg.), Berlin 1975, S, 179-191; Böcker, F., Kaiser, A., Merkle, E., Praxistest für multivariate Verfahren, in: asw, Heft 4, 1976, S. 32-43; Böcker, F., Die Bestimmung der Kaufverbundenheit von produkten, in: Schriften zum Marketing, Dichtl, E., Böcker, F. (Hrsg.), Bd. 7, Berlin 1978; Merkle, E., Die Erfassung und Nutzung von Informationen über den Sortimentsverbund in Handelsbetrieben, in: Schriften zum Marketing, Dichtl, E., Böcker, F. (Hrsg.), Bd. 11, Berlin 1981; Hauzeneder, R., Der Sortimentsverbund im Einzelhandel, Diss. München 1975; Eckhardt, K., Sonderangebotspolitik in Warenhandelsbetrieben - Eine empirische Studie, Wiesbaden 1976; MüllerHagedorn, L., Das Problem des Nachfrageverbundes in erweiterter Sicht, in: ZfbF 1978, S. 181-193; Engelhardt, W.H., Erscheinungsformen und absatzpolitische Probleme von Angebots - und Nachfrageverbunden, in: ZfbF 1976, S. 77-90; Hruschka, M., Bestimmung der Kaufverbundenheit mit Hilfe eines probabilistischen Meßmodells, in: $\mathrm{ZfbF}$ 5/1991, S. 419-434.

26 Eine endgültige Festlegung eines "optimalen" Koeffizienten ist bisher nicht erfolgt. Dies erscheint auch wenig sinnvoll, da es sich um situationsabhängig bedingte $\mathrm{Zu}-$ fallsvariablen handelt. Daher wird auf die Erläuterung einzelner Koeffizienten verzichtet.

$27 \mathrm{Zu}$ Anwendungsbereichen von Verbundmessungsergebnissen vgl. Kapitel C.5. 
- Planung zu bewerbender Artikel, 28

- Umsatzverbund von Haupt- und Nebenprodukt, 29

- Preisfestlegung, 30

- Plazierungsentscheidungen. 31

Gemeinsam ist den unterschiedlichen empirischen Ansätzen, daß sie auf der Basis von Daten eines einzelnen Kaufaktes durchgeführt werden. Ihre Aussagefähigkeit beschränkt sich somit auf primär situativ geprägte Informationen. Elemente von Verbundwirkungen, die erst innerhalb eines Zeitraumes wirken, sowie Auswirkungen von Entscheidungen auf den Verbund im Zeitablauf sind nicht kontrollierbar.

Dieses Problem kann mit Hilfe der Analyse von Kundenkartendaten gelöst werden, da der Kunde mit der Gesamtheit seiner Käufe bei einer Geschäftsstätte sowohl innerhalb eines Kaufaktes als auch innerhalb mehrerer Kaufakte erfaßt wird. 32

28 Vgl. Eckhardt, K., Die Sonderangebotspolitik in Warenhandelsbetrieben - Eine empirische Studie, Wiesbaden 1976.

29 Vgl. Hruschka, H., Folgegeschäfte - zur Bestimmung der Umsatzverbundenheit von Haupt- und Zusatzprodukten mit Hilfe der Polynominal-Lag-Regression, in: Marketing ZFP 1983 , S. 165-169.

Vgl. Simon, H., Preismanagement, wiesbaden 1982.

31 Vgl. Müller-Hagedorn, L., Heidel, B., Plazierungspolitik nach dem Verbundkonzept im stationären Einzelhandel Eine Wirkungsanalyse, in: Marketing ZFP, 1/1989, S. 19 ff.

32 Eine Nichterfassung ist nur möglich, wenn der Kunde aus unbestimmten Gründen bar zahlt. Dies erscheint jedoch unwahrscheinlich, da zum Beispiel erst bei bestimmten Kaufsummen Rabatte wirksam werden. 


\subsection{Probabilistische Meßmodelle als Grundlage der Verbund- analyse}

Einen Ansatz zur Erfassung der Kaufverbundenheit stellt ein probabilistisches Meßmodell dar. Dabei ist das Ziel, die Beschränkungen der Verbundanalyse auf die Interpretation von Paarkäufen aufzuheben. Das bedeutet, daß der gleichzeitige Kauf der Güter $y_{1}, y_{2}$ und $y_{3}$ aufgelöst wird in die Paarkäufe: 33

$$
\begin{aligned}
Y_{1}+y^{2}+y^{3}= & y_{1}+y^{2} \text { und } \\
& y^{2}+y^{3} \text { und } \\
& y^{1}+y^{3}
\end{aligned}
$$

Diese Paarkäufe werden dann - multipliziert mit einem Gewichtungskoeffizienten $c_{i}$ - in eine Häufigkeitsmatrix eingebracht. $c_{i}$ drückt dabei die Konstante des Kaufaktes $i$ aus, die sich aus der Anzahl der im Kaufakt i gekauften Güter wie folgt ergibt:

$$
c_{i}=\frac{1}{m_{i}-1}
$$

wobei $m_{i}$ die Anzahl der im Kaufakt i gekauften Waren angibt.

Die Einführung eines solchen Faktors ist notwendig, damit in der entstehenden Häufigkeitsmatrix die zeilen- und spaltensummen jeweils der Gesamtanzahl der Kaufakte entspricht und nicht die in einem Kaufakt mit vielen anderen Gütern erworbenen Waren durch die vielen Kombinationsmöglichkeiten überbewertet werden. $c_{i}$ stellt somit eine Normierungskonstante dar.

$33 \mathrm{Zu}$ einer detaillierten Beschreibung der Vorgehensweise in dieser Untersuchung vgl. Kap. B.3.; zu diesem Vorgehen z.B. die Untersuchung von Böcker; vgl. Böcker F., Die Bestimmung der Kaufverbundenheit von Produkten, a.a.o. 
Die Häufigkeitsmatrix, die in ihren Dimensionen durch die untersuchten Güter aufgespannt wird, ist dabei symmetrisch. 34 Dies bedeutet eine Gleichsetzung des Kaufpaares $y_{1}$ $/ y_{2}$ mit dem Kaufpaar $y_{2} / Y_{1}$. Ausgangspunkt weiterführender Analysen ist dann diese zweidimensionale Betrachtungsweise.

Hruschka unterstellt für dieses Meßmodell einen Informationsverlust, da die Mehrdimensionalität des einzelnen Kaufaktes verlorengeht. 35 Dies ist bei einer Analyse der zweidimensionalen Matrix auf den Grundlagen multivariater Verfahren zwar nur bedingt nachzuvollziehen, da alle Beziehungen $z$ wischen den einzelnen Gütern eines Kaufaktes abgebildet und Beziehungen $z$ wischen den Kaufpaaren ermittelt werden. Trotzdem entsteht ein gewisser Informationsverlust durch die vernachlässigung der Verbindung zwischen den Kaufpaaren. Um diesen Informationsverlust zu vermeiden, entwickelt Hruschka ein relativ komplexes, mehrdimensionales Meßmodell.

Für jeden Kaufakt wird dabei eine mehrdimensionale Kontingenztabelle aufgestellt, die mit den Daten für den Kauf und Nicht-Kauf von Gütern aufgefüllt wird. Mit Hilfe sog. loglinearer Modelle 36 können dann die Abhängigkeiten in solchen mehrdimensionalen Kontingenztabellen überprüft werden. 37

$34 \mathrm{Zu}$ einer detaillierten Darstellung bzgl. Symmetrie und Asymmetrie von Verbundwirkungen siehe Kapitel 2.21 dieser Untersuchung.

Vgl. Hruschka, H., Bestimmung der Kaufverbundenheit mit $\mathrm{Hilfe}$ eines probabilistischen Meßmodells, in: ZfbF, $43 / 5 / 1991$, S. $418 \mathrm{ff}$.

Vgl. zu loglinearen Modellen z.B. Bishop, Y.M.M., Fienberg, S.E., Holland, P.W., Discrete Multivariate Analysis-Theory and Practice, 0.0., 1975.

37 Vgl. zu diesem Vorgehen z.B. Hamerle, A., Tutz, G., zusammenhangsanalysen in mehrdimensionalen Kontingenztabellen, in: Fahrmeier, L., Hamerle, A. (Hrsg.), Multivariate statistische Verfahren, Wien 1983 S. 473 ff. 
Für den Fall eines Kaufaktes mit drei Gütern erhält Hruschka ein saturiertes dreidimensionales loglineares Modell, das soviele Parameter aufweist, wie die zugrundeliegende Kontingenztabelle zellen besitzt. ${ }^{38}$ Daraus leitet er folgende Modellgleichung ab. 39

$$
\begin{aligned}
I_{n}\left(P\left(y_{1}, y_{2}, y_{3}\right)\right)= & u_{0}+u_{1} y_{1}+u_{2} y_{2}+u_{3} Y_{3}+u_{12} y_{1} Y_{2}+ \\
& u_{13} Y_{1} y_{3}+u_{23} y_{2} Y_{3}+u_{123} Y_{1} Y_{2} Y_{3}
\end{aligned}
$$

Dabei ist $u_{0}$ die logarithmierte erwartete Häufigkeit eines Nichtkaufs aller drei Warengruppen. Die übrigen Faktoren messen jeweils die durch die entsprechenden Güterkäufe (z.B. $u_{1}$ für $Y_{1}$ ) bzw. Güterkaufkombinationen bedingten Abweichungen der logarithmierten erwarteten Zellenbesetzung von $u_{0}$ und bei der Güterkaufkombination noch die Abweichung zu den Effekten der niederen ordnung, wobei die höchste ordnung jeweils durch den Kauf aller Güter bestimmt ist.

Mittels einiger Umformungen und Ableitungen wird die komplexe Gleichung in ein multivariates Logitmodel140 überführt, das weniger Parameter enthält als das entsprechende loglineare Modell. Es stellt letztlich ein Korrelationsmodell für binäre Variablen dar.

Auf den Gleichungen dieser Logit-Modelle baut Hruschka sein probabilistisches system auf, indem er zunächst für jede Wa-

38 Das bedeutet $2^{3}=8$ Parameter.

39 Vgl. Hruschka, H., Bestimmung der Kaufverbundenheit mit Hilfe eines probabilistischen Meßmodells, a.a.o., s. 422 .

40 Vgl. zu multivariaten Logitmodellen Guilkey, D.K., Schmidt, P., Some Small Sample Properties of Estimators and Test statistics in the Multivariate Logit Model, in:

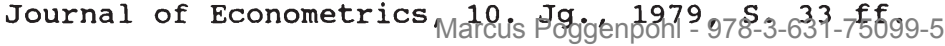


rengruppe Einzelgleichungen aufstellt. ${ }^{41}$ Dabei messen die Parameter der Gleichung eines Gutes für die Käufe anderer Güter den zusammenhang zwischen diesen Käufen. Sie zeigen die bedingte Abhängigkeit zwischen dem abhängigen und dem unabhängigen Gut auf, die als Kaufverbund interpretiert wird. 42

Aus diesen Gleichungen je untersuchtem Gut wird eine Modellsuche für die verbunde je untersuchtem Gut entwickelt. In einem weiteren Schritt werden die Einzelmodelle in ein Gleichungsmodell für alle betrachteten warengruppen überführt, aus denen dann das probabilistische Kaufverbundmodell besteht.

Bei der Modellsuche muß sich Hruschka allerdings, bedingt durch die hohe Anzahl möglicher Kombinationen und damit Modelle, auf ein Grobsuchverfahren beschränken. ${ }^{43}$ Dies ist deshalb als kritisch zu bewerten, weil die Heuristik nicht unbedingt optimale Lösungen garantiert. Auch die Beschränkung auf Effekte niederer ordnung bzw. auf Effekte höherer ordnung, die in den paarweisen Betrachtungen einen Grenzwert überschreiten, zeigt, $d a \beta$ aus pragmatischen Gründen ein Informationsverlust akzeptiert wird. Dies ist vor allem vor dem Hintergrund der zielsetzung, die auf die Behebung von Informationsverlusten ausgerichtet ist, kritisch. Schließlich ist die praktische Anwendung eines derartig komplexen Modells mit umfangreichen Gleichungssystemen in einem Han-

41 Vgl. Hruschka, H., Bestimmung der Kaufverbundenheit mit Hilfe eines probabilistischen Meßmodells, a.a.o., s. 424 .

Vgl. Hruschka, H., Bestimmung der Kaufverbundenheit mit Hilfe eines probabilistischen Meßmodells, a.a.O., S. 424 .

$43 \mathrm{Zu}$ diesem Suchverfahren vgl. Hruschka, H., Bestimmung der Kaufverbundenheit mit Hilfe eines probabilistischen Meßmodells, a.a.0., S. $426 \mathrm{f}$. 
delsunternehmen sehr fraglich, da es in Durchführung und Interpretation eines spezialisten bedarf. Denn zusätzlich zum komplexen Modell bedarf es bei Modellsuche und Interpretation "diverser statistischer Tests und deskriptiver Anpassungsmaße für Logitmodelle". 44

Darüber hinaus besteht keine Möglichkeit der Anwendung des erstellten probabilistischen Modells auf die Problemstellung des Nachfrageverbundes. Auch für die geplante Einbeziehung demographischer Daten bestehen kaum Ansatzpunkte.

\subsection{Ermittlung eines Bekanntheitsverbundes zwischen Abtei- lungen durch Faktorenbildung}

Ein Beispiel für die Anwendung eines faktorenanalytischen Vorgehens zur Ermittlung von Verbundbeziehungen stellt die Untersuchung von Heemeyer dar. ${ }^{45}$ ziel der studie zum Bekanntheitsverbund ist es, ergänzend zu Verbundanalysen, die auf Kaufdaten beruhen, Verbundbeziehungen aufzudecken, die im Bereich des nicht beobachtbaren Verhaltens liegen, sondern auf psychographischen Dimensionen des Konsumentenverhaltens beruhen. 46

44 Hruschka, H. Bestimmung der Kaufverbundenheit mit Hilfe eines probabilistischen Meßmodells, a.a.O., s. 431.

45 Vgl. Steffenhagen, H., Heemeyer, H., Grannemann, R., Bekanntheitsverbund zwischen Warenhausabteilungen - Eine psychographische Verbundanalyse, AP Nr. 81/08 des Instituts für Wirtschaftswissenschaften der RWTH Aachen, Lehrstuhl für Betriebswirtschaftspolitik und Marketing, Aachen 1981 .

46 Vgl. Heemeyer, H., Psychologische Marktforschung im Einzelhandel, Entwicklung und Test einer operationalen Befragungs- und Auswertungskonzeption, Wiesbaden 1981; Steffenhagen, H., Heemeyer, H., Grannemann, R. , Bekanntheitsverbund zwischen Warenhausabteilungen, a.a.0., s. 2 ; Eckhaardt, K., Sonderangebotspolitik in Warenhandelsbetrieben, a.a.0., S. $26 \mathrm{ff}$. 
Grundlage für die Untersuchung ist die Überlegung, daß psychographische Größen wie die Einkaufsstättenbekanntheit und das Einkaufsstättenimage Steuerungsgrößen der Einkaufsstättenwahl darstellen. ${ }^{4}$ Somit stellt der Bekanntheitsverbund hierarchisch eher eine Vorstufe und Einflußgröße des Kaufund Nachfrageverbundes dar.

Die Studie wird auf der Abteilungsebene durchgeführt. Die Befragung erfaßt den Bekanntheitsverbund anhand der aktiven und der passiven Bekanntheit, wobei nur ja/nein-Anworten möglich sind. Somit ergeben sich als Ausgangsbasis für die empirische Auswertung rein dichotome Variablen, wie sie auch bei den meisten Untersuchungen des Kaufverbundes vorliegen. zudem kann auch bei dieser Befragung nur ein symmetrischer Verbund abgeleitet werden, da gerade beim konstrukt Bekanntheit keine Ursache-Wirkungsbeziehung zwischen zwei genannten Abteilungen unterstellt werden kann.

Die Daten werden in eine Häufigkeitsmatrix übertragen und einer Korrelationsanalyse unterzogen. Die Ergebnisse dieser Untersuchung, die ebenfalls in Matrixform vorliegen, bilden dann die Ausgangsdaten für die Durchführung einer Faktorenanalyse, die mit dem ziel der Ermittlung von Verbundclustern von Abteilungen durchgeführt wird. ${ }^{48}$

Insgesamt ist das dieser Studie zugrundeliegende Analyseverfahren für die Verbundforschung als angemessen anzusehen. Für die Ableitung des Kaufverbundes lassen sich jedoch die bei der studie gewonnenen Erkenntnisse nicht verwenden.

47 Vgl. Heemeyer, H., Psychologische Marktforschung im Einzelhandel, a.a.O.; Heinemann, M., Einkaufsstättenwahl und Firmentreue des Konsumenten, a.a.o.

Vgl. Steffenhagen, H., Heemeyer, H., Grannemann, R., Bekanntheitsverbund zwischen Warenhausabteilungen, a.a.o., S. $18 \mathrm{ff}$. 
Selbst die postulierte Hinweisfunktion des Bekanntheitsverbundes für einen zukünftigen Kaufverbund 49 erscheint nicht zwingend. Vor allem eine fehlende Untersuchung konkreter Beziehungen zwischen Bekanntheit und Kauf untersagt eine Übertragung der Ergebnisse.

Darüber hinaus ist es fraglich, ob die vorliegende Befragungstaktik ausreichend objektive Inputdaten abbildet. 50 Das empirische Vorgehen erscheint beispielhaft für weitere Forschungen im Bereich des Bekanntheitsverbundes und aufgrund der analogen Eingangsstruktur der Daten bei Kaufverbundanalysen auch für den Kaufverbund. Für den Bereich des Nachfrageverbundes allerdings erscheint das Instrumentarium nicht als ausgereift.

1.23 Die Transformation von Raufverbundhäufigkeitsmatrizen mittels Regression, Korrelation und Anwendung von Ähnlichkeitsmaßen

Um die Verbundbeziehungen innerhalb eines Sortiments empirisch auswerten zu können, werden unterschiedliche Verfahren angewendet. Diese richten sich unter anderem nach dem skalenniveau der Ausgangsdaten. Grundsätzlich werden die Käufe in eine Matrix übertragen, die in ihren Dimensionen jeweils durch die betrachteten Güter aufgespannt wird, so daß in den Zellen jeder Kauf zweier Güter festgehalten werden kann. Dabei können sich nominal, ordinal oder intervallskalierte Da-

49 Vgl. Steffenhagen, H., Heemeyer, H., Grannemann, R., Bekanntheitsverbund zwischen Warenhausabteilungen, a.a.o., S. 4 .

50 Die objektivität der Ausgangsdaten wird z.B. durch das overreporting, Ja-Sager-Effekte sowie andere Intervieweffekte gefährdet. Vgl. Nieschlag, R., Dichtl., E., Hörschgen, H., Marketing, 13. Aufl., Berlin 1983, S. 134; dieselben, Marketing, ein entscheidungsorientierter Ansatz, 7. Aufl., Berlin 1974, S. 502. 
ten ergeben. 51 Diese Matrizen müssen zur Feststellung von Verbundbeziehungen transformiert werden.

Im Falle ordinalskalierter Daten eignet sich grundsätzlich der Einsatz von Rangkorrelations- und Rangregressionsverfahren. 52 Bei intervallskalierten Daten können, je nachdem ob asymmetrische oder symmetrische Verbundbeziehungen unterstellt werden, andere Verfahren eingesetzt werden. Für symmetrische Matrizen ist die Korrelationsanalyse geeignet, für asymmetrische Matrizen dagegen die Regressionsanalyse. 53 In der überwiegenden $\mathrm{Zahl}$ der Fälle werden für die empirische Analyse nominalskalierte Daten verwendet, die dann die Anzahl der vorkommenden Verbundkäufe widerspiegeln und durch Eintragen in die Zellen eine Häufigkeitsmatrix der Verbundkäufe bilden.

Die Verbundwirkungen zwischen zwei Gütern werden mit Hilfe von Ähnlichkeitsmaßen, die auch als Ähnlichkeitskoeffizienten bezeichnet werden, erfaßt. Um diese Ähnlichkeitsmaße anwenden zu können, werden die Kombinationsmöglichkeiten der beiden betrachteten Güter aus der Häufigkeitsmatrix in sogenannte Vierfeldertafeln überführt. 54 Dabei wird je ein Feld von Konsumenten gebildet, die

- sowohl Gut 1 als auch Gut 2,

- Gut 1 aber nicht Gut 2,

- Gut 2 aber nicht Gut 1 und

- keines der beiden Güter kaufen.

51 Vgl. Kapitel B.2.25 dieser Untersuchung.

52 Vgl. z.B. Lienert, G.A., Verteilungsfreie Methoden der Biostatistik, Bd. 1, 2. Aufl., Meisenheim 1973.

Vgl. Kapitel B.2.23 dieser Untersuchung.

54 Vgl. Stahl, P., Verbundwirkungen im sortiment, a.a.o., S. 159; Böcker, F., Die Bestimmung der Kaufverbundenheit von Produkten, a.a.o., S. 102.; Merkle, E., Die Erfassung und Nutzung von Informationen über den Sortimentsverbund in Handelsbetrieben, a.a.0., S. $53 \mathrm{f}$. 
In der Literatur werden eine Reihe unterschiedlicher Ähnlichkeitsmaße diskutiert. ${ }^{55}$ Eine Übersicht über die auf Nominalskalen beruhenden Faktoren gibt Abbildung 9.56 Einigkeit herrscht darüber, daß die stärke der Ähnlichkeit als Maß für die stärke einer Verbundbeziehung gelten soll.

\begin{tabular}{|c|c|c|c|}
\hline KOEFFIZIENT & & MÄNGEL & EIGNUNG \\
\hline $\begin{array}{l}\text { TETRACHORISCHER } \\
\text { KOEFFIZIENT }\end{array}$ & $r_{\text {tet }}$ & $\begin{array}{l}\text { VERLANGT NORMALVERTEILTE } \\
\text { AUSGANGSDATEN }\end{array}$ & NEIN \\
\hline $\begin{array}{l}\text { YULE- } \\
\text { KOEFFIZIENT }\end{array}$ & & $\begin{array}{l}\text { NEIGT ZU EXTREMWERTEN, DAHER } \\
\text { WENIG TRENNSCHARF } \\
\text { STATISTISCHE VORAUSSETZUNGEN } \\
\text { UNBEKANNT }\end{array}$ & $\begin{array}{l}\text { BEDINGT } \\
\text { JA }\end{array}$ \\
\hline $\begin{array}{l}\text { PHI- } \\
\text { KOEFFIZIENT }\end{array}$ & & $\begin{array}{l}\text { VORAUSSETZUNGEN DER } \boldsymbol{\chi}^{2} \text {-VERTEI- } \\
\text { LUNG; NICHT NORMIERT, DA } \varphi_{\operatorname{mAX}}<1 \\
\text { VORAUSSETZUNGEN DER } \\
\chi^{2} \text {-VERTEILUNG }\end{array}$ & $\begin{array}{l}\text { NEIN } \\
\text { JA }\end{array}$ \\
\hline $\begin{array}{l}\text { HAMANN- } \\
\text { KOEFFIZIENT }\end{array}$ & $\mathbf{Z}$ & $\begin{array}{l}\text { NICHT NORMIERT, DA MEIST } \\
Z_{\text {MIN }}>>-1 ; E(Z) \text { SCHWANKT }\end{array}$ & NEIN \\
\hline $\begin{array}{l}\text { KOEFFIZIENT VON } \\
\text { SOKAL/SNEATH }\end{array}$ & & $\begin{array}{l}\text { NICHT NORMIERT, DA MEIST } \\
\boldsymbol{\delta}_{\text {MIN }}>>0 ; E(\delta) \text { SCHWANKT }\end{array}$ & NEIN \\
\hline $\begin{array}{l}\text { RUSSEL-RAO- } \\
\text { KOEFFIZIENT }\end{array}$ & & $\begin{array}{l}\text { NICHT NORMIERT, DA MEIST } \\
\boldsymbol{\delta}_{\text {MAX }}^{\prime}<<1 ; E\left(\delta^{\prime}\right) \text { SCHWANKT }\end{array}$ & NEIN \\
\hline $\begin{array}{l}\text { FRY- } \\
\text { KOEFFIZIENT }\end{array}$ & & $\begin{array}{l}\text { OHNE LOGISCHE FUNDIERUNG, } \\
\text { NICHT NORMIERT }\end{array}$ & NEIN \\
\hline $\begin{array}{l}\text { ÄHNLICHKEITS- } \\
\text { KOEFFIZIENT } \\
\text { VON JACCARD U.A. }\end{array}$ & $\psi$ & $\begin{array}{l}\text { NICHT NORMIERT, DA MEIST } \\
\psi_{\text {MAX }}<1 ; E(\psi) \text { SCHWANKT } \\
\text { NICHT NORMIERT; UNBESTIMMT } \\
\text { STATISTISCHE VORAUSSETZUNGEN } \\
\text { UNBEKANNT }\end{array}$ & $\begin{array}{l}\text { NEIN } \\
\text { NEIN } \\
\text { BEDINGT }\end{array}$ \\
\hline
\end{tabular}

Abb. 9: Auf Nominalskalen basierende Verbundkoeffizienten (vgl. Merkle, E., Die Erfassung und Nutzung von Informationen über den Sortimentsverbund in Handelsbetrieben, a.a.0., S. 56)

55 Vgl. Böcker, F., Die Bestimmung der Kaufverbundenheit von Produkten, a.a.0., S. $94 \mathrm{ff.;}$ Merkle, E., Die Erfassung und Nutzung von Informationen über den sortimentsverbund in Handelsbetrieben, a.a.0., s. $40 \mathrm{ff}$.

56 Die darüber hinaus gehenden Faktoren für andere skalenniveaus werden nicht aufgefürt, da diese Untersuchung letztendich mit nominalskalierten Daten arbeitet. $\mathrm{zu}$ einer ausführlichen Diskussion von skalierung und Koeffizienten vgl. Böcker, F., Die Bestimmung der Kaufverbundenheit von Produkten, a.a.0., s. 92 ff. 
Besonders problematisch ist bei der Untersuchung von Verbundbeziehungen mit Hilfe von Ähnlichkeitsmaßen, daß es keine endgültigen Aufschlüsse über die optimalen Ähnlichkeitskoeffizienten gibt. Auch die Arbeit von Böcker, die sich intensiv mit solchen Faktoren beschäftigt hat, kommt zu keinen eindeutigen Ergebnissen. 57 Die Fülle der Einflußfaktoren auf die Ausprägung von Verbundwirkungen ist sehr hoch und in ihrer wirkung kaum festzulegen. ${ }^{8}$ Auf der anderen Seite sind die stichproben der empirischen Berechnungen aber relativ klein. Auch die von Böcker als optimal erkannten Koeffizienten haben sich bei der praktischen Anwendung nicht bewährt.59 Insgesamt lassen sich folgende Kritikpunkte zusammenfassen: 60

- Die Koeffizienten sind stark abhängig vom Verdichtungsgrad der Daten.

- Die Koeffizienten sind abhängig davon, ob Teilsortimente oder das Gesamtsortiment betrachtet werden.

- Die Koeffizienten sind abhängig vom statistischen Erwartungswert.

Auch wenn bei der Wahl eines Koeffizienten ein Ähnlichkeitsmaß entwickelt wird, das die Werte der Zelle der Vierfeldertafel, die die Fälle enthält, in denen keines der untersuchten Güter gekauft wurde, nicht einbezieht, läßt sich kein optimales Maß finden. Zwar ist ein solcher Koeffizient in

57 Vgl. Böcker, F., Die Bestimmung der Verbundenheit von Produkten, a.a.0., S. $102 \mathrm{ff}$.

58 Siehe dazu auch Kapitel 2.1 dieser Untersuchung.

59 Vgl. Merkle, E., Die Erfassung und Nutzung von Informationen über den Sortimentsverbund in Handelsbetrieben, a.a.o., s. 53 .

60 Vgl. Merkle, E., Die Erfassung und Nutzung von Informationen über den Sortimentsverbund in Handelsbetrieben, a.a.O., S. 82 f. Marcus Poggenpohl-978-3-631-75099-5 
seinen Abhängigkeiten wesentlich robuster 61 als die in $\mathrm{Ab}-$ bildung 9 enthaltenen Faktoren, doch ergeben sich andere Nachteile. So muß z.B. die symmetrievoraussetzung der Verbundmessung aufgegeben werden, wenn nicht mehr die summe der Käufe der beiden Güter als Basis der Koeffizientenrechnung herangezogen wird.

\subsection{Die Verwendung multivariater Analysemethoden bei der Verbundanalyse}

Grundsätzlich besteht in umfangreichen sortimenten bei einer Verbundanalyse die Notwendigkeit, die vielfältigen Verbundbeziehungen zu strukturieren. Dabei sollen auch die zusammenhänge, die in den Verbundmatrizen (umgewandelte Häufigkeitsmatrix der Kaufverbunde) bestehen, aufgedeckt werden. Im allgemeinen erscheinen alle statistischen Verfahren geeignet, die zusammenhänge und Abhängigkeiten in umfangreichen Datenstrukturen abbilden können. Dabei werden in der Praxis in der Hauptsache die Faktorenanalyse, die clusteranalyse und die mehrdimensionale skalierung (MDS) angewendet. Die Anwendungen führen zu tragfähigen Ergebnissen und aussagefähigen Interpretationen, so daß im folgenden kurz die in unterschiedlichen Untersuchungen erprobten Verfahren skizziert werden.

\subsection{Faktorenanalyse}

Mit Hilfe der Faktorenanalyse sollen in der Sozialwissenschaft allgemein aus einer vielzahl von Variablen einige zentrale Faktoren abgeleitet werden, die den ursprünglichen

61 Vgl. Merkle, E., Die Erfassung und Nutzung von Informationen über den Sortimentsverbund in Handelsbetrieben, a.a.O., s. $84 \mathrm{f}$. 
Sachverhalt abbilden. 62 Im Falle der Kaufverbundmessung sind Warengruppen, aus denen Güter gekauft werden, zu Obergruppen zusammengefaßt worden. Da aus der Häufigkeitsmatrix dabei bereits Verbundmaße eingehen, geben die gebildeten Faktoren Verbundgruppen der Warengruppen wieder. Dabei stellen die Faktorladungen die Grundlage für die Interpretation der $\mathrm{Zu}-$ sammensetzung der Faktoren und somit der Verbundgruppen dar. Problematisch wird die Interpretation dann, wenn bestimmte Warengruppen sehr hoch auf unterschiedliche Faktoren laden. Dieser Effekt kann vor allem bei Warengruppen mit sehr hoher Kauffrequenz bestehen.

Bei bisherigen empirischen Untersuchungen wurde die Faktorenanalyse durchaus als brauchbares verfahren zur verbundanalyse bewertet, 63 wenn die Eingangsdaten das erforderliche Skalenniveau aufweisen. Sowohl Böcker als auch Merkle äußern in diesem Zusammenhang Bedenken. Die mit unterschiedlichen Ähnlichkeitsmaßen behandelten Häufigkeitsmatrizen sollen danach kein ausreichendes skalenniveau aufweisen. 64 steffenhagen dagegen begründet die grundsätzliche Einsetzbarkeit der

Vgl. zur Faktorenanalyse z.B. Backhaus, K., Erichsen, B., Plinke, W., Schuchard-Ficher, C., Weiber, R., Multivariate Analysemethoden, 4. Aufl., Berlin et al. 1987, S. $67 \mathrm{ff.;}$ Meffert, H., Marketingforschung und Käuferverhalten, Wiesbaden 1992, S. 257 ff.; Meffert, H., Marktforschung, Wiesbaden 1986, S. 83 ff.; Bleymüller, J., Faktorenanalyse, Münster 1978; Überla, K., Faktorenanalyse, Berlin, Heidelberg, New York 1972; Revenstorf, D., Lehrbuch der Faktorenanalyse, stuttgart 1976; Ost, F., Faktorenanalyse, in: Fahrmeier, L., Hamerle, A. (Hrsg.), Multivariate statistische Verfahren, Berlin 1984, S. 575 ff.; Weiber, R., Faktorenanalyse, st. Gallen 1984 .

Vgl. Merkle, E., Die Erfassung und Nutzung von Informationen über den Sortimentsverbund in Handelsbetrieben, a.a.0., S. 102; Stahl, P., Verbundwirkungen im sortiment, a.a.o., s. 71 ff.; Böcker, F., Die Bestimmung der Kaufverbundenheit von Produkten, a.a.0., s. $161 \mathrm{f}$.

64 Vgl. Böcker, F., Die Bestimmung der Kaufverbundenheit von Produkten, a.a.O., S. 162. 
Faktorenanalyse damit, daß sie als Verfahren, das einen linearen Zusammenhang zwischen Variablen unterstellt, auch nominalskalierte Daten verarbeiten kann. 65

Die Diskrepanz gegenüber einem geforderten, zumindest intervallskalierten Datenniveau ist jedoch dadurch als überbrückt anzusehen, daß Verfahren wie z.B. Diskriminanz- und Faktorenanalysen nicht sehr empfindlich gegenüber unzureichenden Meßniveaus der Ausgangsdaten sind. 66 zudem sind Häufigkeiten durchaus als Ausgangsmaterial für Faktorenanalysen nutzbar.

Die grundsätzliche Anwendbarkeit dieses Verfahrens zeigt sich auch darin, daß steffenhagen durchaus den Hypothesen entsprechende aussagefähige Faktoren erhält.67 Auch stahl erhält gute Ergebnisse, die in ihrer struktur den Ergebnissen einer Anwendung der multidimensionalen skalierung auf die gleichen Eingangsdaten sehr stark gleichen. 68

\subsection{Multidimensionale skalierung}

Die multidimensionale skalierung wird allgemein zur Verbundmessung als geeignet angesehen. ${ }^{69}$ Grundsätzlich soll mit

65 Vgl. Steffenhagen, H., Heemeyer, H., Grannemann, R., Bekanntheitsverbund zwischen Warenhausabteilungen, a.a.o., S. 13 .

66 Vgl. Kerlinger, F.N., Grundlagen der Sozialwissenschaften, Bd. 1, deutsche Übersetzung, Weinheim-Basel 1979, S. $674 \mathrm{f}$.

67 Vgl. Steffenhagen, H., Heemeyer, H., Grannemann, R., Bekanntheitsverbund zwischen Warenhausabteilungen, a.a.o., S. $26 \mathrm{ff}$.

68 Vgl. Stahl, P., Verbundwirkungen im sortiment, a.a.o., S. 168 .

69 Zur multidimensionalen skalierung vgl. Backhaus, K., Erichson, B., Plinke, W. , Schuchard-Ficher, C., Weiber, R., Multivariate Analysemethoden, a.a.0., s. 317 ff.; 
Hilfe der multidimensionalen Skalierung erreicht werden, daß objekte als Punkte in einem möglichst niedrig dimensionierten Raum so abgebildet sind, daß die räumliche Nähe die Ähnlichkeit der objekte wiedergibt. Im Falle der verbundmessung wird die Ähnlichkeit als Verbundbeziehung interpretiert. Dabei ist die Verbundbeziehung um so stärker, je näher sich die einzelnen objekte geometrisch sind. Durch eine wahrnehmbare Gruppenbildung könnten dabei wiederum Verbundgruppen identifiziert werden.

Mit Hilfe der multidimensionalen skalierung können aufgrund ihrer Anschaulichkeit direkt Hinweise für die räumliche Planung der Warenpräsentation, für eine sinnvolle Warensystematik und auch für die Ermittlung von Verbrauchertypen abgeleitet werden. ${ }^{70}$ Die Anwendung der multidimensionalen Skalierung führt zu aussagefähigen Ergebnissen. ${ }^{71}$ Böcker wendet die multidimensionale skalierung allerdings nur in Teilsortimenten an. 72

Bezüglich des Meßniveaus der Ausgangsdaten sind im Falle der multidimensionalen Skalierung keine Anforderungen gegeben, denen die Daten der Häufigkeitsmatrix nicht entsprechen können, da sie nur ordinal skaliertes Niveau erfordert.

Borg, I., Anwendungsorientierte Multidimensionale Skalierung, Berlin, Heidelberg, New York 1981; Meffert, H., Marketingforschung und Käuferverhalten, a.a.0., s. 313 ff.; Meffert, H., Marktforschung, a.a.O., s. 126 ff.; Dichtl., E., Schobert, R., Mehrdimensionale Skalierung Methodische Grundlagen und betriebswirtschaftliche Anwendungen, München 1979.

70 Vgl. Merkle, E., Die Erfassung und Nutzung von Informationen über den Sortimentsverbund in Handelsbetrieben, a.a.0., S. 105 .

71 Vgl. Stahl, P., Verbundwirkungen im sortiment, a.a.o., S. 166 ff.; Böcker, F., Die Bestimmung der Kaufverbundenheit von Produkten, a.a.0., S. $162 \mathrm{ff}$.

72 Vgl. Böcker, F., Die Bestimmung der Kaufverbundenheit von Produkten, a.a.0., S. $168 \mathrm{ff}$. 
Einen anderen Einwand aber bringt Böcker in seiner Untersuchung vor. ${ }^{73}$ Er weist darauf hin, daß die untersuchten Objekte (in diesem Fall Verbundkäufe) keine von vornherein getrennten Affinitätsklumpen aufweisen, da diesen bei der späteren Interpretation der Ergebnisse ein zu hoher Erklärungsanteil zugesprochen wird. Da sortimente aber häufig in abgrenzbare sortimentsbereiche aufteilbar sind, wird der Schluß gezogen, es solle nur eine Anwendung der multidimensionalen skalierung innerhalb dieser Teilsortimente erfolgen.

Dieser Schlußfolgerung kann nur bedingt gefolgt werden, da bei dieser Vorgehensweise sortimentsbereichsübergreifende Verbundbeziehungen verlorengehen. Je nach zielsetzung der Untersuchung ist dies jedoch nicht akzeptabel. Grundsätzlich ist zwar eine Bestätigung bestehender Sortimentsgruppierungen kaum von Interesse, doch sind gerade Verbundbeziehungen, die diese Gruppierungslogik sprengen, besonders $z u$ beachten und haben für das Handelsmarketing hohe Relevanz.

\subsection{3 clusteranalyse}

Die clusteranalyse hat grundsätzlich das ziel, eine Anzahl von objekten entsprechend ihrer Ähnlichkeit, die durch die Ausprägungen verschiedener Merkmale festgestellt wird, zu Gruppen oder Klassen zusammenzufassen. ${ }^{74}$ Dabei sollen die

73 Vgl. Böcker, F., Die Bestimmung der Kaufverbundenheit von Produkten, a.a.0., S. 168 .

74 Vgl. zur clusteranalyse Backhaus, K., Erichson, B., Plinke, W., Schuchard-Ficher, C., Weiber, R., Multivariate Analysemethoden, a.a.O., s. 115 ff.; Meffert, H., Marketingforschung und Käuferverhalten, a.a.0., S. 267 ff.; Meffert, H., Marktforschung, a.a.o., s. $91 \mathrm{ff.;}$ Steinhausen, D., Langer, K., Clusteranalyse, Berlin, New York 1977 . 
Gruppen in sich möglichst homogen und untereinander möglichst heterogen sein. Bisher wurde die clusteranalyse zur Bildung von Verbundgruppen nur von Merkle eingesetzt. ${ }^{75}$ Als Ausgangsbasis werden dabei allerdings keine Häufigkeitsmatrizen in transformierter oder standardisierter Form herangezogen. Eingangsmaterial für die Anwendung der Clusteranalysen bilden sogenannte Warengruppenprofile.

Dabei wird jeder Warengruppe eine Kaufverbundhäufigkeit für jede andere Warengruppe zugeordnet. Dies stellt anschaulich gesprochen die zeilenweise Abbildung einer Häufigkeitsmatrix dar. Diese Profile werden als der individuelle Verbund jeder Warengruppe angesehen. Durch Überführung dieser individuellen Profile in eine clusteranalyse werden dann Warengruppencluster gebildet. Dabei stellen die Warengruppen die objekte dar und die Verbundkaufhäufigkeiten mit den anderen Warengruppen die Merkmalsausprägungen. Auf diese Weise kommt Merkle zu aussagekräftigen Verbundgruppen. 76

Dieses Vorgehen kann auch für andere Zielsetzungen transformiert werden. Werden die individuellen Kaufprofile nicht für jede einzelne Warengruppe aufgestellt, sondern im Rahmen der Analyse des Nachfrageverbundes für jeden einzelnen Kunden, so lassen sich neue zielsetzungen im Rahmen der Verbundforschung realisieren. Wenn jedem Kunden ein individuelles Kaufprofil zugeordnet wird, so stellen nicht mehr die Warengruppen die zu gruppierenden objekte dar, sondern die Kunden. Auf diese Weise ist es möglich, Kundengruppen anhand von Verbundgrößen $z u$ bilden. Dieser Aspekt wird im Rahmen

75 Vgl. Merkle, E., Die Erfassung und Nutzung von Informationen über den Sortimentsverbund in Handelsbetrieben, a.a.0., S. $105 \mathrm{ff}$.

76 Vgl. Merkle, E., Die Erfassung und Nutzung von Informationen über den sortimentsverbund in Handelsbetrieben, a.a.O., S. $114 \mathrm{ff}$. 
der Erforschung der Zielgruppenbildung anhand von Verbundkäufen erneut aufgegriffen. 77

\section{Die Erfassung von Kauf- und Nachfrageverbund im Handel}

\subsection{Einflußgrößen und Bestimmungsfaktoren des Rauf- und Nachfrageverbundes}

Vor einer weiterführenden Darstellung und Diskussion der Verbundmessung ist es zur Fundierung einer Hypothesenbildung notwendig, das system unterschiedlicher Einflußgrößen und Bestimmungsfaktoren des Kauf- und Nachfrageverbundes $z u$ analysieren. Auf die Entstehung von Verbundbeziehungen wirken, wie bereits bei der Erklärung der Divergenzen zwischen dem generellen und dem unternehmensspezifischen Nachfrageverbund verdeutlicht wurde, eine Fülle von Faktoren, die z.T. nur schwer durch das Unternehmen kontrollierbar sind. Die Einflußgrößen, die durch das Handelsunternehmen kontrolliert werden können, sind in der Gruppe angebotsbezogener Einflußgrößen zusammengefaßt.

Eine weitere Gruppe umfaßt diejenigen Größen, die von der Seite des Konsumenten auf die Ausgestaltung des Kauf- und Nachfrageverbundes wirken. Diese Größen können vom Handelsunternehmen nicht kontrolliert werden. Die dritte zentrale Gruppe von Einflußgrößen umfaßt die wettbewerbsbezogenen Aspekte, die ebenfalls durch das Handelsunternehmen nicht direkt beeinflußt werden können.

Hinzu tritt eine vierte Gruppe, die allerdings nur indirekt auf den Kauf- und Nachfrageverbund wirkt, indem sie die zentralen Bestimmungsfaktoren beeinflußt. Hierzu zählen

77 Vgl. Kapitel 3.22 dieser Untersuchung. 
- herstellerinduzierte Größen ${ }^{78}$,

- bauliche Voraussetzungen und

- allgemeine Umweltfaktoren.

Diese Bereiche werden unter den indirekten Einflußgrößen des Kauf- und Nachfrageverbundes subsumiert. Einen Überblick über die gewählte systematisierung und die den Einflußbereichen zuzuordnenden Größen gibt Abbildung 10.

Die vorgestellte Systematisierung stellt eine idealtypische Trennung von Einflußbereichen dar, die eine optimale Möglichkeit zur Ableitung von Hypothesen zu Wirkungsbeziehungen im Kauf- und Nachfrageverbund bietet. Anhand solcher Hypothesen ist eine fundierte Untersuchung des Kauf- und Nachfrageverbundes möglich. In diesem zusammenhang werden auch Einflußgrößen aufgenommen, die im Rahmen dieser Untersuchung nicht empirisch überprüfbar sind. Zum einen ist dadurch eine umfassende Darstellung des komplexen Einflußgrößensystems möglich. Zum anderen können offene Forschungsfelder abgeleitet werden.

Die idealtypische Trennung der Einflußgrößen bedeutet allerdings keine Unabhängigkeit der drei zentralen Faktorengruppen. So können Gegebenheiten des Wettbewerbs die angebotsbezogenen Größen beeinflussen. Ist in einem Sortimentsbereich $z . B$. eine sehr hohe wettbewerbsintensität vorhanden, so kann dies $z u$ einem Rückzug des untersuchten Handelsunternehmens aus diesem Sortimentsbereich führen.

Ebenso kann die Ausgestaltung angebotsbezogener Faktoren auf die konsumentenbezogenen Einflußgrößen wirken. Wird z.B. in einem Sortimentsbereich, in dem ein Kunde unsicher bezüglich seiner Kaufentscheidung ist, keine Beratung angeboten, so

78 Vgl. Merkle, E., Die Erfassung und Nutzung von Informationen über den Sortimentsverbund in Handelsbetrieben, a.a.0., S. $16 \mathrm{f}$. 


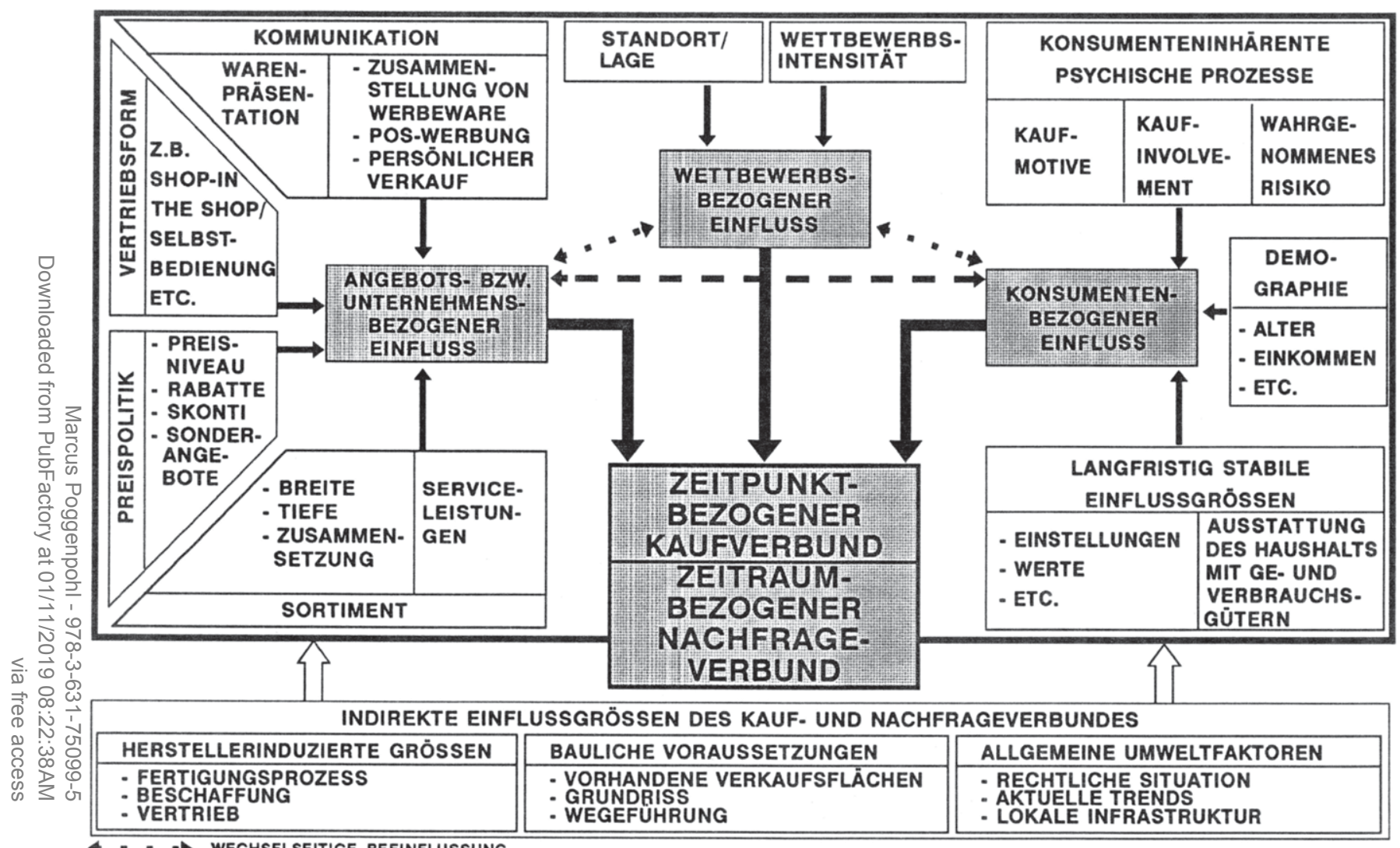

Abb. 10: Einflußgrößen des Kauf- und Nachfrageverbundes 
kann dies zu einer völlig veränderten Wahrnehmung der Kaufsituation 79 führen.

Darüber hinaus beeinflussen konsumentenbezogene Faktoren die Ausgestaltung wettbewerbs- und angebotsbezogener EinflußgröBen. Eine Veränderung in grundlegenden Einstellungen 80 muß letztendich vom Handelsunternehmen und vom Wettbewerb berücksichtigt werden. So ist beispielsweise seit geraumer zeit bei den Konsumenten ein gestiegenes Umweltbewußtsein festzustellen, das vor allem die Einstellungen zu den ökologischen Konsequenzen des Konsums umfaßt. ${ }^{81}$ Der damit verbundenen ökologieorientierung des Konsumentenverhaltens ist von seiten der Industrie und des Handels durch die Bereitstellung eines umweltverträglichen produktangebots bzw. eine ökologieorientierte sortimentsgestaltung Rechnung $\mathrm{zu}$ tragen.

Die Untersuchung von Wirkungsbeziehungen verschiedener Einflußgrößen auf die Ausgestaltung von Kauf- und Nachfrageverbundstrukturen wird durch die simultane wirkung aller Einflußgrößen erschwert. Deshalb erscheint eine isolierte Betrachtung der Einflußgrößengruppen nur wenig sinnvoll. Zur Lösung dieser Problematik bietet es sich an, wirkungshypo-

79 Eine Diskussion des Begriffs der Kaufsituation findet im Kapitel der konsumentenbezogenen Einflußgrößen Kapitel B. 2.11 statt.

80 Zum Begriff der Einstellung als Prädisposition eines Konsumenten zur Reaktion auf Reize vgl. Meffert, H., Marketing, a.a.0., S. 151; insbesondere $\mathrm{zu}$ Einstellungen als Grundlage für kognitive und konative Prozesse auch Kroeber-Riel, W., Konsumentenverhalten, 4. Aufl., München 1990, S. 49-54, S. 162 ff.; Müller-Hagedorn, L., Das Konsumentenverhalten - Grundlagen für die Marktforschung, Wiesbaden 1986, S. 79 ff., S. 182 ff.; Trommsdorff, V., Konsumentenverhalten, stuttgart 1989, S. 121 ff.

81 Zur Darstellung und Analyse des Umweltbewußtseins als ein Einstellungskonstrukt vgl. Monhemius, K.Ch., Umweltbewußtes Kaufverhalten von Konsumenten, Frankfurt a.M. 1993, S. 1 ff. 
thesen zu formulieren, die einzelne Einflußgrößen aufgreifen und gleichzeitig alle anderen Größen als konstant festlegen (ceteris-paribus-Bedingung). Eine Variation der untersuchten Einflußgröße kann dann isoliert auf ihre Auswirkungen auf den Kauf- und Nachfrageverbund untersucht werden.

\subsection{Angebots- bzw. unternehmensbezogene Einflußgrößen des Kauf- und Nachfrageverbundes}

Die angebotsbezogenen Einflußgrößen auf den Kauf- und Nachfrageverbund können anhand der vier Marketing-Mix-Bereiche systematisiert werden. 82

Das sortiment als Gegenstand des ersten Marketing-Mix-Bereiches bildet zum einen die restriktive Größe für Verbundbeziehungen. Zum anderen wird sowohl durch die Breite als auch durch die Tiefe des Sortiments ${ }^{83}$ die Möglichkeit einer Verbundbeziehung erst gegeben. Nicht angebotene Sortimentsbereiche können auch keinen Verbund induzieren, selbst wenn eine verwendungsbezogene Affinität besteht.

In analoger Weise wirkt auch die zusammensetzung des Sortiments. Dabei sind in diesem Zusammenhang vor allem abgrenzbare Sortimentsbereiche angesprochen. So kann das Handelsun-

82 Vgl. Meffert, H., Marketing - Grundlagen der Absatzpolitik, 7. Aufl., Wiesbaden 1986, S. 115 ff.; Wehrle, F., Strategische Marketingplanung in Warenhäusern, Schriften zum Markting, Bd. 1, Meffert, H. (Hrsg.), Frankfurt a.M. 1981, S. 190; Patt, P.-J., Strategische Erfolgsfaktoren im Einzelhandel, Schriften zu Marketing und Management, Bd. 14, Meffert, H. (Hrsg.), Frankfurt a.M. 1988, S. 62 ff.

$83 \mathrm{Zu}$ den Begriffen sortimentsbreite und -tiefe vor allem als Grundlage von Verbundbeziehungen vgl. Stahl, P., Verbundwirkungen im Sortiment, a.a.0., s. 5; Böcker, F., Die Bestimmung der Kaufverbundenheit von Produkten, a.a.0., s. 21 . 
ternehmen durch die Aufnahme neuer oder die Auslistung bestehender sortimentsbereiche Verbundbeziehungen beeinflussen bzw. neue Verbundbeziehungen ermöglichen. 84 Dabei ist die Möglichkeit gegeben, daß ein neuer sortimentsbereich nur interne Verbundbeziehungen hervorruft bzw. durch eine Aufwertung des gesamten sortiments neue Verbundbeziehungen im Restsortiment auslöst. Dieser Effekt ist bspw. im Serviceangebot zu beobachten, wenn durch die individuelle Kundenbetreuung der Kunde das gesamte sortiment verändert wahrnimmt. Darüber hinaus kann ein Mangel an Serviceleistungen auch zu einer negativen Sortimentswahrnehmung und zur Auflösung von Verbundbeziehungen führen. Daher ist bei der Elimination oder Variation bestehender sortimente und Serviceangebote den bestehenden Verbundwirkungen besondere Beachtung $\mathrm{zu}$ schenken. 85

Im Bereich der preispolitik sind insbesondere das allgemeine Preisniveau, Rabatte, Skonti und Sonderangebote von Interesse. In bezug auf das allgemeine preisniveau ist festzuhalten, daß mit zunehmendem Preisniveau die Qualitätswahrnehmung steigt 86 und die Rationalität des Kaufs zunimmt. ${ }^{87} \mathrm{Da}-$ bei neigt der Konsument dazu, zielkäufe nicht mit einer vielzahl von anderen Käufen $z u$ verbinden. Rabatte können insbesondere als Mengenrabatte verbundauslösend wirken. skonti sind dagegen erst bei einer Erfassung von zeitraumbe-

$\mathrm{Zu}$ den Strategien der Aufnahme und Aufgabe von Sortimentsbereichen vgl. Wehrle, F., Strategische Marketingplanung in Warenhäusern, a.a.O., S. $195 \mathrm{ff}$.

85 Vgl. Wehrle, F., strategische Marketingplanung in Warenhäusern, a.a.O., S. 206 .

86 Vgl. zu diesem Aspekt des Preisniveaus Merkle, E., Die Erfassung und Nutzung von Informationen über den Sortimentsverbund in Handelsbetrieben, a.a.0., S. $23 \mathrm{f}$.

87 Vgl. zu diesem Themenbereich auch Diller, H., Die Wirkung von Verkäufen unter Einstandspreisen im Lebensmitteleinzelhandel, a.a.0., S. $409 \mathrm{ff}$. 
zogenen Verbunden von Bedeutung, indem sie die Wahrscheinlichkeit zum Wiederbesuch der Einkaufsstätte erhöhen. ${ }^{88}$

$\mathrm{Zu}$ den kommunikationspolitischen Instrumenten im Handel als angebotsbezogene Einflußgrößen zählen insbesondere die Werbung am Pos, die Zusammenstellung von Werbeware in Angebotspaketen, der persönliche Verkauf sowie die Warenpräsentation ${ }^{89}$. So kann das Handelsunternehmen durch die Zusammenstellung von Waren innerhalb einer Werbemaßnahme beim Kunden Verbundkäufe anregen und somit die verbundstruktur beeinflussen. Einen analogen Effekt kann die Pos-Werbung auslösen.

Im Rahmen des persönlichen Verkaufs ist es dem Verkaufspersonal möglich, durch gezielte Beratung eines kunden und dessen kontinuierliche Führung durch mehrere sortimentsbereiche Verbundkäufe anzuregen und gezielt zu steuern. Dies kann einen erheblichen Einfluß auf die Verbundbeziehungen haben. Die Beeinflussungsmöglichkeiten sinken jedoch mit dem Bedienungsgrad bzw. der Beratungsintensität. 90 Die Warenpräsenta-

$88 \mathrm{Zu}$ den Auswirkungen von Sonderangeboten auf die Verbundbeziehungen im Sortiment vgl. Eckhardt, K., Die Sonderangebotspolitik in Warenhandelsbetrieben - eine empirische studie, a.a.o.

89 Zur Analyse der Kommunikationspolitik in Handelsbetrieben vgl. Patt, P.-J., Strategische Erfolgsfaktoren im Handel, a.a.0., S. $65 \mathrm{ff}$. sowie die dort zitierte Literatur; Hansen, U., Absatz- und Beschaffungsmarketing des Einzelhandels, Teil 2, Göttingen 1976, S. $422 \mathrm{ff}$.

Zur Beratungsintensität und deren operationalisierung vgl. Bierbaum, M., Leistung und Leistungsbedingungen im Einzelhandel, Rationalisierungs-Gemeinschaft des Handels beim RKK e.V. Köln (Hrsg.), Köln 1979, S. 30 ff. Bierbaum stellt dabei ein Verhältnis von Vorwahlfläche (Verkaufsfläche) und Verkaufspersonal als Maß für Beratungsintensität dar. 
tion kann insbesondere über die Plazierungsentscheidung ${ }^{91}$, aber auch über die Belebung der Warenpräsentation mit Erlebniselementen ${ }^{92}$ beeinflussend auf den Kaufverbund wirken. Der Einfluß der Warenpräsentation im Bereich des zeitraumbezogenen Konstruktes Nachfrageverbund ist nicht zuletzt aufgrund der Tatsache, daß die Warenpräsentation i.d.R. kurzfristig verändert wird, eher gering.

Die Vertriebsform stellt in erster Linie eine Rahmenbedingung und restriktive Größe für andere Einflußaspekte dar. So gibt es bei reiner Selbstbedienung keine Einflußmöglichkeit des persönlichen Verkaufs. Ebenso ist in supermärkten meist das Preisniveau als eher niedrig anzusetzen und es finden überwiegend Versorgungskäufe statt. Somit besteht auch durch die Vertriebsform ein Einfluß auf Breite und Tiefe des Sortiments.

Allerdings bestehen auch innerhalb einer vertriebsform (z.B. Warenhaus) Möglichkeiten, alternative Konzepte $z u$ erproben (z.B. Shop-in-the-shop). ${ }^{93}$ Durch die Bildung im Prinzip abgeschlossener Verkaufseinheiten entsteht ein völlig anderes Bild im Wahrnehmungsraum des Kunden, als dies bei einer durchgehenden Verkaufsfläche gegeben ist. Hierdurch werden sowohl innerhalb des abgegrenzten sortimentsbereiches als auch $z$ wischen den verschiedenen Bereichen Verbundbeziehungen verändert. Jedoch ist die Messung dieser Änderung durch feh-

91 Vgl. Müller-Hagedorn, L., Heidel, B., Plazierungspolitik nach dem Verbundkonzept im stationären Einzelhandel Eine Wirkungsanalyse, a.a.O., S. $19 \mathrm{ff}$.

92 Zur wichtigkeit von Kauferlebnissen vgl. silberer, G., Die Bedeutung und Messung von Kauferlebnissen im Handel, in: Handelsforschung 1989, Jahrbuch der Forschungsstelle für den Handel, Berlin (FfH) e.V., Trommsdorff, $V$. (Hrsg.), Wiesbaden 1989, S. 59 ff.

93 Vgl. Medla, K., Shop-in-the-shop ein Konzept der Angebotspräsentation, a.a.o. 
lende Vergleichsmessungen bei der Ursprungssituation kaum möglich.

Die Arbeitshypothese zu angebotsbezogenen Faktoren des Raufverbunds lautet:

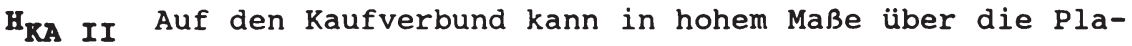
zierung der Abteilungen innerhalb der Geschäftsstätte Einfluß genommen werden.

Zur Überprüfung dieser Hypothese wird ein Vergleich der ermittelten Kaufverbundstrukturen mit einem Lageplan der Abteilungen innerhalb der Geschäftsstätte vorgenommen.

\subsection{Ronsumentenbezogene Einflußgrößen des Kauf- und Nach- Erageverbundes}

Die Gruppe der Einflußgrößen auf den Kauf- und Nachfrageverbund von seiten des Konsumenten ist besonders vielfältig und komplex. Daher ist es notwendig, eine problemorientierte Strukturierung der Größen vorzunehmen. Vor dem Hintergrund der situativen Ausrichtung des Kaufverbundes und der zeitraumbezogenen orientierung des Nachfrageverbundes erscheint es zweckmäßig, auch die konsumentenbezogenen Einflußgrößen nach ihrer kaufsituativen und zeitraumbezogenen wirksamkeit einzuteilen.

Im Hinblick auf die erste Gruppe von konsumentenbezogenen Einflußgrößen ist $z u$ diskutieren, inwieweit Konstrukte und Einflußgrößen aus dem Kaufverhalten bzgl. eines Einproduktkaufs auf Verbundkäufe in der Kaufsituation übertragbar sind. Grundsätzlich besteht zwischen der finalen Kaufentscheidung für ein produkt und einem Verbundkauf kein Unterschied, da auch die Verbundanalyse zunächst von Einzelkäufen ausgeht. Mit der Kombination einzelner Produktkäufe zu Ver- 
bundbeziehungen sowie der Analyse der Beziehungen werden Rückschlüsse auf zusätzliche kaufauslösende Aspekte ermöglicht, die die Affinität der gekauften Produkte zueinander betreffen. Dabei unterliegen die zugrundeliegenden Käufe den gleichen Einflußstrukturen wie Einproduktkäufe, so daß die Erkenntnisse der Kaufverhaltensforschung auch für den Verbundkauf zugrunde gelegt werden können.

$\mathrm{Zu}$ den Größen, die in der aktuellen Kaufsituation einen Verbund beeinflussen können, zählen zum einen konsumenteninhärente psychische Prozesse (z.B. Kaufmotive 94 , wahrgenommene Kaufrisiken ${ }^{95}$ ). Zum anderen sind diejenigen Einflußfaktoren bedeutsam, die durch den kaufort selbst bestimmt sind (z.B. Sortimentszusammensetzung, Warenpräsentation) und daher als "angebotsbezogene Einflußgrößen" bezeichnet werden sollen. Somit beschränken sich die kaufsituativen Einflußfaktoren auf die konsumenteninhärenten Größen.

$94 \mathrm{Zu}$ Motiven als Einflußgröße des Kaufverhaltens vgl. Kroeber-Riel, W., Konsumentenverhalten, a.a.O., S. 148 . Dort werden z.B. Abwechslung, Erfolg und überlegenheit als besonders antriebsstark für den Kauf beschrieben. Diese Größen sind jedoch eher langfristig wirksam. In der Kaufsituation sind eher Motive wie Erfüllung von Qualitätsansprüchen, Preisgünstigkeit etc. von Bedeutung. Grundsätzlich zu Motiven vgl. auch Meffert, H., Marketing, a.a.0., S. 150 .

$\mathrm{Zu}$ Begriff und Modellen des wahrgenommenen Kaufrisikos (perceived risk) vgl. Gemünden, H.G., Wahrgenommenes Risiko und Informationsnachfrage - Eine systematische Bestandsaufnahme der empirischen Befunde, in: MarketingZfP, Heft 1, Febr. 1985, S. 27 ff., Kupsch, P., Hufschmied, P., Wahrgenommenes Risiko und Komplexität der Beurteilungssituation als Determinante der Qualitätsbeurteilung, in: Meffert, H., steffenhagen, H., Freter, H. (Hrsg.), Konsumentenverhalten und Information, Wiesbaden 1979, S. $225 \mathrm{ff.;}$ zu Zwei-Komponenten-Modellen (z.B. Unsicherheit und wichtigkeit oder Unsicherheit und Kauffolgen als Komponenten von Risikomodellen) vgl. den Überblick von Schweiger, H., Mazanec, J., Wiegele, O., Das Modell des "erlebten Risikos" ("percieved risk"). Struktur und operationalisierungskonzepte, in: Der Markt, 60/1976, S. $94 \mathrm{f}$. 
Zur Feststellung, welche psychischen konsumenteninhärenten Prozesse in der Kaufsituation wirksam werden und einen Einfluß auf Verbundkäufe ausüben können, wird auf den Ansatz von Monhemius zurückgegriffen, 96 wonach den Kaufmotiven und wahrgenommenen Kaufrisiken sowie dem Kaufinvolvement während der Kaufsituation eine hohe Kaufverhaltensrelevanz zukommt.97 Vorrangige Begründung für diese Konstruktauswahl ist, $d a \beta$ es sich um psychische prozesse handelt, die den Konsumenten am Point of Sale zum Handeln antreiben und aktivieren. Beschreibt man "Aktivierung" als einen Zustand innerer spannung, der das Individuum in Leistungsbereitschaft versetzt $z u$ denken, $z u$ fühlen und $z u$ handeln, dann werden den Kaufmotiven, den wahrgenommenen Kaufrisiken sowie dem Kaufinvolvement die größten Aktivierungspotentiale zugeschrieben.

Mit den Raufmotiven sind die Bedürfnisse angesprochen, die der Konsument durch Einprodukt- bzw. Verbundkäufe zu erreichen sucht. Derartige Motive, die kaufsituativ wirksam werden können, sind bspw.

- die Erlangung eines Preisvorteils (z.B. durch Produkte im Sonderangebot)

96 Der Untersuchung von Monhemius liegt die Fragestellung zugrunde, welche psychischen Prozesse in der Kaufsituation den Kauf bestimmter - in diesem Fall ökologierelevanter - Produkte beeinflussen können. Aufgrund der bereits erörterten Nähe zwischen kaufsituativ bedingten Einproduktkäufen und Verbundkäufen erweist sich der Ansatz auch für die vorliegende Untersuchung als geeignet. Vgl. Monhemius, K.Ch., Umweltbewußtes Kaufverhalten von Konsumenten, a.a.O., S. $91 \mathrm{ff}$.

$97 \mathrm{Zu}$ einer Abgrenzung dieser drei Antriebskräfte vgl. Kroeber-Riel, W., Konsumentenverhalten, a.a.O., S. 4954 , S. 138 ff. 
- die Käufe von Produkten mit qualitätsgarantie (z.B. bestimmte Markenartikel mit der Hersteller- oder Produktmarke als Qualitätsgaranten)

- die Käufe von Produkten mit dem "Umweltengel" zur Befriedigung des Bedürfnisses nach der Umweltverträglichkeit des Konsums.

Diese und andere Kaufmotive haben einen starken Einfluß auf die produktwahl und damit die Ausgestaltung von Kaufverbundbeziehungen.

Das Raufinvolvement 98 umfaßt das innere Engagement und Interesse, mit dem sich der Konsument dem Einkauf selbst zuwendet und wodurch seine Informationsaufnahme und -verarbeitung während der Kaufsituation beeinflußt wird. Es lassen sich produktbezogenes und situatives Involvement voneinander unterscheiden. Das Produktinvolvement umfaßt das Ausmaß der inneren Auseinandersetzung mit einem Produkt, einer Marke oder auch einer Warengruppe. Mit dem situativen Involvement ist der durch situative Faktoren ausgelöste Zustand der Aktivierung angesprochen. Letzteres ist bspw. der Fall, wenn der Konsument Einkäufe unter zeitdruck ${ }^{99}$ tätigen muß und sich in einem inneren spannungszustand zwischen "keine zeit für Produktsuche" und "dringendem produktbedarf" befindet.

$\mathrm{Zu}$ unterschiedlichen Arten und Grundlagen des Involvement vgl. Deimel, K., Grundlagen des Involvement und Anwendung im Marketing, in: Marketing ZFP, Heft 3, 1989, S. $153 \mathrm{ff}$. Das situative Involvement kennzeichnet dabei den inneren Zustand der Aktivierung, der durch situationsbedingte Faktoren ausgelöst wird. $\mathrm{Zu}$ einer ausführlichen Diskussion des Kaufinvolvement vgl. auch Monhemius, K.Ch., Umweltbewußtes Kaufverhalten von Konsumenten, Bd. 18 der Schriften zu Marketing und Management, Meffert, H. (Hrsg.), Frankfurt a.M. 1993, S. 113 ff.

Vgl. zum Einfluß des Zeitdrucks auf das Kaufverhalten z.B. Steffenhagen, H., Entscheidungsverhalten unter Zeitdruck, in: DBW, 4/1978, S. 595 ff. 
Weitere Beispiele, die das spektrum des Einflusses von Kaufinvolvement auf den Verbundkauf verdeutlichen, sind:

- aufgrund des starken Interesses an bestimmten Warengruppen (z.B. Textilien) wird eine bestimmte Einkaufsstätte des Handels aufgesucht, wo das Warenangebot den subjektiven Ansprüchen genügt

- bietet eine Warengruppe, der der Konsument ein hohes Produktinvolvement entgegenbringt, die gewünschte sortimentsauswahl und -qualität (z.B. DOB-Warengruppe), so kann es durchaus sein, daß der konsument von dieser auf weitere Warengruppen schließt und einen Verbundkauf tätigt (z.B. HAKA- oder Kinderbekleidungs-Warengruppe).

Anders als Kaufmotive und -involvement, die den Konsumenten in der Kaufsituation $\mathrm{zu}$ bestimmten Käufen veranlassen können, tragen wahrgenommene Kaufrisiken zur subjektiven Verunsicherung bei der Produktwahl bei. So ist es beispielsweise denkbar, daß der Besucher eines bestimmten Handelsgeschäftes von der Qualität des dargebotenen sortiments insgesamt nicht überzeugt ist. Aufgrund des dadurch bestehenden Risikos, daß das gewünschte Produkt nicht seinen Erwartungen entspricht, wird eine andere als die beabsichtigte Kaufentscheidung getroffen. Im Hinblick auf den Einfluß von Kaufrisiken auf den Verbundkauf kann dies etwa dazu führen, daß

- der Konsument aus den Warengruppen seiner zielkäufe nur die ihm bekannten Markenartikel auswählt, da er die Hersteller- oder Produktmarke als Qualitätsgaranten erachtet und hierdurch sein wahrgenommenes Kaufrisiko reduzieren kann. Dadurch kommen keine Verbundkäufe, z.B. zu "NoName"-Produkten zustande.

- Konsumenten, die ein finanzielles Kaufrisiko (d.h. die Gefahr, daß ein zu hoher Preis für den Produktkauf ver- 
langt wird) verspüren, gezielt nach Produkten Ausschau halten, die im Sonderangebot sind und dementsprechende Verbundkäufe tätigen.

- Konsumenten, die ein gesundheitliches Kaufrisiko bei ökologierelevanten produkten verspüren, gezielt nach Produktvarianten suchen, die als gesundheits- bzw. umweltverträglich gekennzeichnet sind. Dann entstehen verstärkt Verbundbeziehungen mit derartigen produkten.

Insgesamt besteht eine enge Wechselwirkung der konsumenteninhärenten situativen Einflußgrößen mit den angebotsbezogenen Einflußfaktoren am Point of sale. Da die Gegebenheiten innerhalb der Geschäftsstätte (z.B. Produktplazierung und präsentation sowie Beratung) den aktivierenden Einfluß der psychischen prozesse auslösen bzw. verstärken können, stellt die gezielte Untersuchung der konsumenteninhärenten Einflußstrukturen die Grundlage für eine Verhaltensbeeinflussung durch das Handelsmarketing dar. Diese Verhaltensbeeinflussung ermöglicht es wiederum, das Zustandekommen von Kaufverbundbeziehungen zu fördern.

Kaufmotive, -involvement und -risiken stellen insgesamt psychische prozesse dar, die während der Kaufsituation den einzeln betrachteten - Verbundkauf beeinflussen und insofern erklären können (Ein-Zeitpunkt-Betrachtung). Für die zeitraumbezogene Analyse von Verbundkäufen ist ein Konstrukt heranzuziehen, das nicht nur kurzfristig, sondern längerfristig wirksam ist und einen Einfluß auf das Kaufverhalten über einen längeren Zeitraum hinweg ausüben kann. Hierbei bietet sich das Einstellungskonstrukt an, da Einstellungen als "innere Bereitschaft (Prädisposition) eines Individuums, auf bestimmte stimuli der Umwelt konsistent positiv oder ne- 
gativ zu reagieren"100, über einen längeren Zeitraum stabil bleiben und Verbundkäufe nachhaltig beeinflussen können. objekte der Einstellungen können Sachen, Personen oder Denkobjekte (z.B. bestimmte Themen) sein. ${ }^{101}$ In der vorliegenden Untersuchung können vor allem Abteilungen, die Geschäftsstätte selbst, aber auch Einzelartikel, Warengruppen oder das gesamte Sortiment Gegenstand von Einstellungen sein kann.

Ein Beispiel für die Auswirkung von Einstellungen bzw. Einstellungsänderungen auf die Ausgestaltung von Verbundbeziehungen ist die inzwischen ablehnende Haltung gegenüber Naturpelzen. Durch die Einstellungsänderung werden alle bestehenden Verbundbeziehungen dieses Sortimentsbereichs aufgelöst, wobei gleichzeitig neue verbundkaufmuster entstehen. In letzter Konsequenz führt eine Auflösung dieser Verbundbeziehungen auch zur Aufgabe des Sortimentsbereichs. 102

Neben den Einstellungen als längerfristig wirksame psychische Prozesse, die Verbundkäufe determinieren können, stellt die Ausstattung des Haushaltes mit Ge- und Verbrauchsgütern eine weitere zentrale Einflußgröße dar. Vor allem bei langlebigen Konsumgütern (High-Tech-Geräte; Möbel etc.) bildet ihr Vorhandensein im Haushalt eine restriktive Größe für das Zustandekommen von Verbundbeziehungen. Wenn sich ein solches

100 Meffert, H., Marketing, a.a.0., S. 151. Vgl. Werner, J., Einstellungen zum Produkt und Einstellungen zum Produktbereich als Grundlage einer Konsumententypologie, a.a.0., S. 158. $\mathrm{Zu}$ Werten als Grundlage zur Bildung von Einstellungen vgl. Dahlhoff, D., Wertorientierungen von Verbrauchern, in: Handwörterbuch der angewandten Psychologie, Bd. 3: Markt und Umwelt, Landsberg 1981, S. 130. Dahlhoff geht dabei davon aus, daß die Werte die zeitlich stabilste Grundeinstellung von Konsumenten wiedergeben und sie somit auch für Einstellungen bestimmend sind.

101 Vgl. ebenda.

$102 \mathrm{Vgl}$. die Ausführungen in Kapitel B.2.1 dieser Arbeit. Marcus Poggenpohl - 978-3-631-75099-5 
Produkt im Haushalt befindet, so ist nicht davon auszugehen, daß es innerhalb seiner Nutzungsdauer durch einen Kauf- oder Nachfrageverbund bedingt neu erworben wird.

Eine Sonderstellung im Bereich der konsumentenbezogenen Einflußgrößen nehmen die sozioökonomischen charakteristika der Konsumenten ein. Sie können sowohl kaufsituativ als auch längerfristig wirksam werden. So kann das vorhandene finanzielle Budget als restriktive Größe im situativen Bereich angesehen werden, da es die möglichen Käufe begrenzt. Andererseits sind Einstellungen häufig mit bestimmten Ausprägungen sozioökonomischer Kriterien verknüpft. So ist beispielsweise das Merkmal Alter eine Erklärungsgröße für Einstellungsänderungen gegenüber bestimmten Warengruppen (z.B. Textilien, Möbel).

Die sozioökonomischen Merkmale nehmen darüber hinaus in dieser Untersuchung eine Sonderstellung ein, da sie bei der Verbundanalyse auf der Basis von Kundenkarteninformationen als Daten vorhanden sind. Durch die empirische Datenverfügbarkeit ergeben sich Ansatzpunkte für ihre Anwendung, die die Ausgestaltung des Handelsmarketing betreffen. Dies wird bei der Formulierung von Arbeitshypothesen berücksichtigt. 103

Die Arbeitshypothese $z u$ konsumentenbezogenen Faktoren des Nachfrageverbunds lautet:

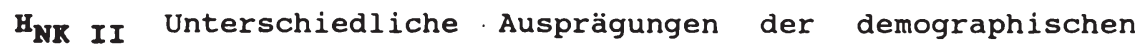
Variablen "Alter" und "Einkommen" haben keine Auswirkungen auf die grundsätzliche struktur der mit der Nachfrageverbundanalyse ermittelten Bedarfsgruppen. Lediglich die Kaufintensitäten dieser Gruppen variieren.

$103 \mathrm{Vgl}$. die Ausführungen in Kapitel B.2.15 dieser Arbeit. Marcus Poggenpohl - 978-3-631-75099-5 
Zur Überprüfung dieser Hypothese sind zwei Schritte erforderlich: Zum einen werden die ermittelten Nachfrageverbundstrukturen in den verschiedenen Alters- und Einkommensklassen miteinander verglichen. Zum anderen werden die Kaufintensitäten der bestimmenden Abteilungen überprüft.

\subsection{Wettbewerbsbezogene Einflußgrößen des Kauf- und Nach- frageverbundes}

Die wettbewerbsbedingten Einflußgrößen des Kauf- und Nachfrageverbundes sind der standort bzw. die Lage der Geschäftsstätte104, in der der Verbund gemessen werden soll und die Wettbewerbsintensität. Der standort kann auf der einen seite eher auf die makrogeographische Lage bezogen werden.

Dabei sind als grundsätzliche Unterscheidung die Ansiedlung einer Geschäftsstätte in der Innenstadt oder zum anderen eine Ansiedlung "auf der grünen Wiese" von Bedeutung. Eine Ansiedlung in der Innenstadt bedingt zum Beispiel, daß in der überwiegenden Zahl der Fälle wegen fehlender Parkmöglichkeiten keine Versorgungskäufe getätigt werden. Umgekehrt ist dann aber auch festzustellen, daß "auf der grünen wiese" wegen des fehlenden Ambiente kaum andere als der Versorgung dienende Käufe getätigt werden. Insofern kann bei der Erfassung eines Vollsortimenters bereits eine Einschränkung möglicher Verbundwirkungen bestehen.

In diesem Zusammenhang ist die Trennung des Handels in Versorgungs- und Erlebnishandel von Bedeutung. Dabei führt die zunehmende Polarisierung zum einen zu unterschiedlichen Sor-

$104 \mathrm{Zu}$ situationsfaktoren von standorten vgl. Wehrle, F., strategische Marketingplanung in Warenhäusern, a.a.o., S. 203 . 
timenten. Der Versorgungshandel deckt den Grundbedarf der Konsumenten $\mathrm{ab}$, während der Erlebnishandel tendenziell höherwertige sortimente vertreibt. Zum anderen bedingt diese Differenzierung in zwei Extreme unterschiedliche Präsentationsarten, d.h. die Präsentation ist im Erlebnishandel z.B. wesentlich aufwendiger und stärker auf die schaffung eines Ambiente ausgerichtet als beim Versorgungshandel. Sowohl das Sortiment als auch die Präsentation haben als angebotsbezogene Größen Einfluß auf die Entstehung von Verbundstrukturen.

Darüber hinaus kann die Zuordnung des Handelsunternehmens zu einem dieser beiden Bereiche auch zur Definition des relevanten Wettbewerbs beitragen. Zumeist steht ein supermarkt als Repräsentant des Versorgungshandels nicht in direktem Wettbewerb mit einem Delikatessengeschäft mit hochwertiger Präsentation und eventuell integrierter Degustation als Vertreter des Erlebnishandels. Der relevante Wettbewerb besteht somit aus Vertretern einer Handelsausrichtung. Eine noch detailliertere Einschränkung ist durch die Konzentration auf Unternehmen der gleichen Betriebsform möglich. $\mathrm{Zu}$ Problemen würde das jedoch z.B. bei der Betriebsform Warenhaus führen, da deren relevanter Wettbewerb auch den Facheinzelhandel umfaßt.

$\mathrm{Zu}$ den standortaspekten ist auch die mikrogeographische Lage zu rechnen. Diese kann durch Unterscheidungen wie z.B. die Lage an einer Fußgängerzone105 gegenüber einem standort an einer Hauptverkehrsstraße beschrieben werden. In diesem $\mathrm{Zu}-$ sammenhang ist auch die Lage der Wettbewerber in die Betrachtung einzubeziehen. Dies fällt zudem in den Bereich der Wettbewerbsintensität.

105 Zur Bedeutung von Fußgängerzonen für den Einzelhandel vgl. z.B. Spannagel, R., Strukturwandel im Einzelhandel durch Fußgängerzonen, in: FfH-Mitteilungen, Januar 1979, S. $1 \mathrm{ff}$. 
Bei der Wettbewerbsintensität ist die Intensität in bezug auf Anzahl und Größe gleichartiger Wettbewerber zu beachten. Bildet ein Vollsortimenter die untersuchte Geschäftsstätte, so ist die Untersuchung der Wettbewerbsintensität nicht nur auf gleichartige Wettbewerber begrenzt (z.B. nur Warenhäuser als Wettbewerber von Warenhäusern), sondern es sind in jedem einzelnen Warengruppenbereich der gesamte relevante Wettbewerb, z.B. Fach- und Einzelhandel, einzubeziehen. Über eine Einschätzung von Größe und Umsatz der Wettbewerber ist dabei aber auch deren Preis- oder Servicepolitik von zentralem Interesse.

Insgesamt können auch die wettbewerbsbezogenen Einflußgrößen nur einen Erklärungshintergrund für die Interpretation der spezifischen Geschäftsstättenverbundbeziehungen bilden und besitzen selbst keine direkte verbundbestimmende Bedeutung. Dennoch sind die wettbewerbsbezogenen Einflußgrößen fester Bestandteil der Kontextdimensionen einer Geschäftsstätte und zum besseren Verständnis bestimmter Verbundbeziehungen, die von plausiblen Hypothesen abweichen, notwendig.

\subsection{Indirekte Einflußgrößen des Kauf- und Nachfrageverbun- des}

$\mathrm{Zu}$ den herstellerinduzierten Größen gehören z.B. Beschaffung und Vertrieb auf Herstellerebene. Dabei fällt unter Beschaffung z.B. die Beschaffung von Rohstoffen mit ihren Restriktionen und kosteninduzierten Rahmenbedingungen. Zum Vertrieb zählt die wahl der jeweiligen Vertriebsform ebenso wie die Festlegung von Preisen und sonstigen Konditionen. 106

106 Vgl. Merkle, E., Die Erfassung und Nutzung von Informationen über den Sortimentsverbund in Handelsbetrieben, a.a.0., s. 17 . Marcus Poggenpohl - 978-3-631-75099-5 
Diese herstellerbezogenen Größen haben nur dann eine direkte wirkung auf den Kauf- und Nachfrageverbund eines Individuums, wenn jede Zwischenstufe des Vertriebs ausgeschaltet wird und eine direkte Hersteller-Endabnehmer-Beziehung besteht. In anderen Fällen spiegeln sich diese Einflußgrößen im Bereich der angebots- bzw. unternehmensbezogenen Einflußgrößen des untersuchten Handelsunternehmens wider. Dabei werden insbesondere die Punkte Sortiment und Preispolitik berührt.

Eine weitere indirekte Auswirkung auf den Kauf- und Nachfrageverbund wird von den baulichen Gegebenheiten ausgeübt. So bilden z.B. die vorhandenen Verkaufsflächen Restriktionen für den Handel. Sie begrenzen die Möglichkeit der Warenpräsentation ebenso wie Breite und Tiefe des Sortiments. So ist zum einen auf knappen Verkaufsflächen keine hochwertige warenpräsentation mit einer Einbindung von Erlebniselementen möglich. Auch ist bei knappen Flächen nur ein begrenztes Sortiment in Breite und Tiefe zu führen.

Weiterhin sind der Grundriß der Verkaufsflächen und die Wegführung $z u$ betrachten. So sind "verwinkelte" Verkaufsflächen oder sehr langgestreckte Verkaufsflächen nicht gut erreichbar und daher schon von der Anzahl der Kunden, die die Fläche aufsuchen, benachteiligt. In eine vergleichbare Richtung zielt die Wegeführung innerhalb der Verkaufsflächen, die zur Steuerung der Kunden angelegt wird. Sie soll einer gleichmäBigen Auslastung der Verkaufsflächen dienen. Dies ist jedoch nicht immer gegeben. Die Wegeführung wird in diesem zusammenhang nur als indirekte Wirkungsgröße angesetzt, da sie kaum kurzfristig veränderbar ist und somit eher eine Rahmenbedingung als eine direkte Einflußgröße darstellt.

Darüber hinaus bestehen noch Einflüsse durch die allgemeinen Umweltfaktoren. Diese setzen sich aus der rechtlichen Situa- 
tion 107 , aktuellen Trends und der lokalen Infrastruktur zusammen. Stellen die ersten beiden Aspekte eher allgemeine juristisch-kulturelle Rahmenbedingungen dar, so ist die 10kale Infrastruktur eher als spezifisch für jede einzelne Geschäftsstätte anzusehen. Darunter fallen z.B. die Verkehrsanbindung, die Parksituation, die Anbindung an öffentliche Verkehrsmittel, aber auch die ökonomischen Rahmenbedingungen des Standortes wie Kaufkraft, Arbeitslosigkeit etc. Sie bilden damit die individuellen Kontextdimensionen einer Geschäftsstätte.

\subsection{Operationalisierungsansätze bei der Verbundmessung}

Vor einer Erfassung von Verbundbeziehungen in Kauf- und Nachfrageverbund ist es notwendig, unterschiedliche Aspekte der operationalisierung von Verbundkonstrukten zu erfassen und zu diskutieren. Dabei stehen sich die Vor- und Nachteile verschiedener optionen gegenüber und sind vor dem Hintergrund theoretischer und empirisch bedingter Voraussetzungen für die Untersuchung des Kauf- und Nachfrageverbunds festzulegen.

\subsection{Typen von Verbundwirkungen}

Im Bereich der nachfrageinduzierten Verbundwirkungen können wiederum unterschiedliche Typen von Verbundwirkungen identifiziert werden. Diese Typen ergeben sich durch die Anzahl der bei einem Kauf erworbenen Güter und die Ausprägungen des

107 Vgl. Merkle, E., Die Erfassung und Nutzung von Informationen über den Sortimentsverbund in Handelsbetrieben, a.a.o., s. 18 . 
Instrumenteeinsatzes. Der Instrumenteeinsatz soll die Ausprägungen verändert und konstant aufweisen. 108

Durch die Kombination von Güteranzahl pro Kauf und Instrumenteeinsatz ergeben sich vier Typen von Verbundwirkungen, die in Abbildung 11 den Bereichen einer 4-Felder-Matrix zugeordnet werden:

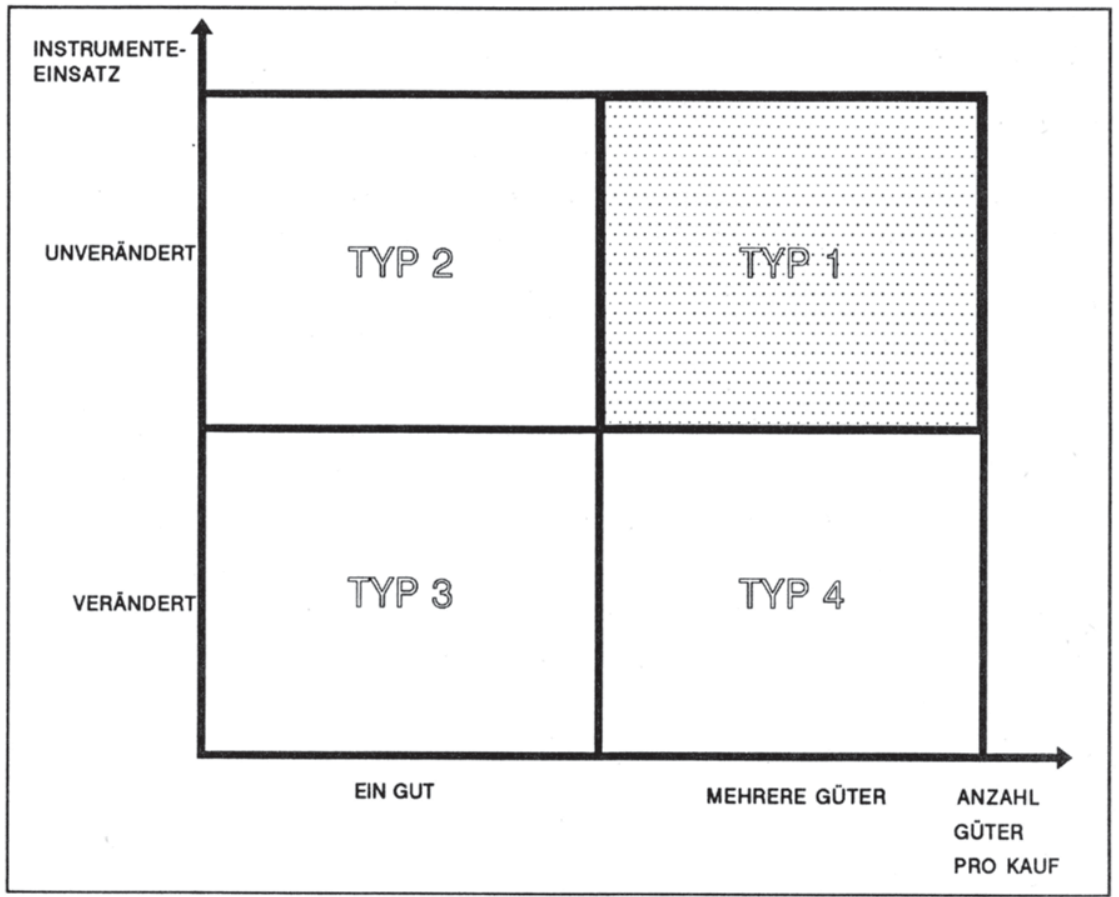

Abb. 11: Typen von Verbundbeziehungen

- Typ 1 ergibt sich aus der Messung der Verbundwirkung bei konstantem Instrumenteeinsatz und mehreren Gütern pro Kauf. Dies wird der im weiteren Verlauf der Untersuchung analysierte Typ sein. Die Notwendigkeit, gerade diesen

$108 \mathrm{Vgl}$. Stahl, P., Verbundwirkungen im Sortiment, a.a.o., S. 53 . 
Typ zu wählen, ergibt sich insbesondere für die Erfassung des Nachfrageverbundes. Dieser stellt eine zeitraumbezogene Größe dar. Je nach untersuchtem zeitraum ist jedoch ein Instrumenteeinsatz kaum isoliert $z u$ erfassen. Somit kann der Instrumenteeinsatz nicht oder nur als extrem komplexe Größe mit hohen Wechselwirkungen zwischen verschiedenen Maßnahmen abgebildet werden. Desgleichen wird bei der Analyse eines bestimmten Konsumenten im Zeitablauf immer ein Kauf mehrerer Güter vorliegen.

- Typ 2 ist durch einen veränderten Instrumenteeinsatz und den Kauf nur eines Gutes gekennzeichnet. Die Käufe nur eines Gutes zu erfassen, kann zum einen dazu dienen, Anteile von Verbundkäufen am Gesamtabsatz eines Gutes zu ermitteln. 109 Dabei stellt der Kauf eines Gutes an sich also nur ein Hilfsmittel zur Erfassung bestimmter Verbundeffekte dar. Darüber hinaus wird jedoch ein weiteres Phänomen erfaßt, das man als Eigenverbund oder Intensivkauf eines Gutes bezeichnen kann. Dieser tritt auf, wenn ein Gut in höherer stückzahl erworben wird. Merkle faßt dies als reflexive Beziehung eines Gutes auf, die z.B. durch einen Verwendungsverbund (z.B. mehrere Batterien zum Betrieb eines Gerätes notwendig) oder die Gewährung von Mengenrabatten ausgelöst werden kann.110 Dieser Eigenverbund zeigt jedoch grundsätzlich keine Abhängigkeiten zwischen unterschiedlichen Gütern oder Warengruppen auf.

Da die Verbundkäufe insgesamt eine vom Konsumenten empfundene Affinität widerspiegeln, werden Käufe mit nur

109 Vgl. Stahl, P., Verbundwirkungen im sortiment, a.a.o., S. 54 .

110 Vgl. Merkle, E., Die Erfassung und Nutzung von Informationen über den Sortimentsverbund in Handelsbetrieben, a.a.0., S. $34 \mathrm{f}$. 
einem Artikel entgegen den Annahmen von Stahl,111 Eckhardt 112 und Merkle 113 im folgenden nicht berücksichtigt. Dies erscheint vor allem deshalb gerechtfertigt, weil die Verbundbeziehungen im folgenden nicht auf einen Kaufakt beschränkt werden, sondern innerhalb eines definierten Zeitraums $z u$ analysieren sind. Dabei werden nur solche Käufer ausgeschlossen, die innerhalb des definierten Zeitraums nur einen Artikel gekauft haben. Bei diesen Käufern ist jedoch auch nicht von bestehenden Verbundbeziehungen auszugehen.

- Typ 3 ergibt sich bei der Betrachtung eines Gutes und unverändertem Instrumenteeinsatz. Dieser Typ ist für eine Erfassung von Verbundeffekten irrelevant, da nicht einmal die wirkung einer Maßnahme (z.B. Sonderangebot) auf den Absatz eines Gutes betrachtet werden kann. Darüber hinaus gelten auch hier die Ausführungen zu Typ 2.

- Typ 4 von Verbundwirkungen entsteht durch die Beobachtung der Käufe mehrerer Güter bei verändertem Instrumenteeinsatz. Für die grundsätzliche Erfassung der wirkungen absatzpolitischer Instrumente auf die Verbundwirkungen im Sortiment ist dieser TYp geeignet. Problematisch wird allerdings schon bei dieser spezifischen Fragestellung die Isolierung einer einzelnen Maßnahme, da dem Kauf- und Nachfrageverbund ein äußerst komplexes Netz von Einflußgrößen gegenübersteht. Damit könnte eine Erfassung allenfalls durch ein Experiment möglich sein.

111 Vgl. Stahl, P., Verbundwirkungen im sortiment, a.a.o., S. 116 .

112 Vgl. Eckhardt, K., Sonderangebotspolitik in warenhandelsbetrieben, a.a.0., S. 210 .

113 Vgl. Merkle, E., Die Erfassung und Nutzung von Informationen über den Sortimentsverbund in Handelsbetrieben, a.a.o., S. 33 . 
Somit wird im weiteren Verlauf der Untersuchung der veränderte Einsatz absatzpolitischer Instrumente grundsätzlich nicht in die Betrachtung einbezogen. Für Verbundwirkungsmessungen wird der Kauf mehrerer Güter vorausgesetzt.

Allerdings ist insbesondere im Hinblick auf zukünftige Forschungsansätze im Rahmen der Verbundforschung auf die Bedeutung einer Erfassung von Einflußgrößen z.B. aus dem Bereich absatzpolitischer Maßnahmen $z u$ verweisen. Bereits bei der Analyse der Einflußgrößen 114 wurde die Bedeutung auch der absatzseitigen Aspekte für die Entstehung von Verbundbeziehungen betont.

Eine Erfassung und Einbeziehung dieser Größen stößt jedoch in der empirischen Forschung aufgrund des benötigten Datenumfangs auf probleme. Kaufauslösende Aspekte wie Sonderangebote sind beispielsweise nur im Rahmen von Befragungen $z u$ erfassen. Die mit dieser Technik verbundenen Probleme sollen hier nicht weiter diskutiert werden. Auch die Auswahl und Festlegung relevanter Einflüsse ist nicht direkt lösbar, da sich ein komplexes Netzwerk von Wechselwirkungen zwischen einzelnen Aspekten ergeben kann. Daher sind isolierte Wirkungen zum Teil nicht $z u$ erfassen. Die angesprochenen Schwierigkeiten wären allerdings im Rahmen einer aufwendigen empirischen Forschung $z u$ beheben.

Darüber hinaus besteht jedoch eine weitergehende Problemstellung, die kaum lösbar ist. Diese besteht in der mangelnden operationalisierbarkeit einiger zentraler Einflußgrößen. So ist es unbestritten, daß z.B. die Atmosphäre und das Ambiente kaufbeeinflussender Faktoren von hoher Bedeutung sind. Es ist jedoch nicht möglich, diese Größen meßbar zu machen. Dies liegt zum einen in ihrer eher abstrakten Natur begründet. Zum anderen spiegeln diese Begriffe selbst

114 Vgl. Kapitel B.2.1 
ein umfassendes Bündel von Eindrücken mit kaum zu erfassenden Synergie- und Beeinflussungseffekten wider.

Insgesamt ist festzustellen, daß die Vernachlässigung absatzpolitischer Maßnahmen zu einem Informationsverlust führt, der bei der Interpretation der Ergebnisse zu berücksichtigen ist. Dennoch erscheint dieses Vorgehen vor dem Hintergrund der empirischen Probleme bei weitgehenden Erfassungsmaßnahmen gerechtfertigt. Bei weiterführenden Forschungen wäre allerdings $z u$ jedem Kauf eine gut operationalisierte Erfassung absatzpolitischer Maßnahmen (z.B. durch eine Codenummer im Kaufdatensatz, die sich aus der EAN-Nummer ergibt) wünschenswert, um eine Verbesserung der Informationslage zu erreichen.

\subsection{2 symmetrische und asymmetrische verbundbeziehungen}

Grundlegend für eine Erfassung von Verbundwirkungen ist auch, ob symmetrische oder asymmetrische Verbundbeziehungen vorliegen. 115 Bei einer asymmetrischen Beziehung geht man davon aus, daß ein Bestandteil des Verbundkaufes den anderen auslöst, wobei die Richtung eindeutig ist. Bei symmetrischen Beziehungen entsteht der Verbundkauf durch nicht erforschbare technische, imagebedingte, verwendungs- oder bedarfsbezogene Affinitäten. Ein Beispiel für eine verwendungsbezogene Affinität ist der Kauf von schwarzem Schuhpflegemittel, der durch den Kauf schwarzer Schuhe ausgelöst wird. Eine technische Affinität kann z.B. in Form von Tennisball und Tennisschläger bestehen etc. Bei den folgenden Analysen wird von symmetrischen Beziehungen ausgegangen, wohingegen $z . B$. bei der Werbeerfolgsanalyse asymmetrische Beziehungen mit

$115 \mathrm{Vgl}$. Böcker, F., Die Bestimmung der Kaufverbundenheit von Produkten, a.a.O., S. 82 f.; Heidel, B., Die Nutzung von Scannerdaten zur Planung der Absatzpolitik von Einzelhandelsbetrieben, a.a.0., s. $113 \mathrm{ff}$. 
dem auslösenden Element der beworbenen Ware postuliert werden können.

Bei symmetrischen Verbundwirkungen ist der Verbund von Gut 1 mit Gut 2 gleich dem Verbund von Gut 2 mit Gut 1 . Dieses Vorgehen wird gewählt, weil die Bestimmung der Richtung einer Verbundwirkung bzw. die Festlegung eines Auslösers für einen Verbundkauf problematisch ist.116 In diesem zusammenhang bleiben letztlich nur Plausibilitätsannahmen.

Zu diesen Annahmen zählt z.B. auch, daß die Richtung von Verbundbeziehungen vom Ausmaß der in einer Warengruppe getätigten Käufe abhängen kann ${ }^{117}$. Dies ist ebenso problematisch wie die Aussage, daß stets das teurere Gut Auslöser ist. Die erste Annahme kann gelten, wenn Güter mit grundsätzlich gleicher Kauffrequenz untersucht werden. Die zweite Annahme ist vor allem bei Untersuchungen auf dem Aggregationsniveau von Abteilungen oder bei Sortimenten mit gravierenden Preisdifferenzen nicht tragfähig.

Auch die besonderen Eigenschaften von Kundenkartendaten führen in diesem Zusammenhang nicht weiter. Zwar könnte aufgrund der Tatsache, daß die Kaufzeitpunkte einzelner Käufe registriert werden, der jeweils früher getätigte Kauf als Auslöser angesetzt werden. Um diese Annahme haltbar zu machen, sind jedoch die zusätzlichen Einflüsse auf die Abfolge von Käufen, wie z.B. die Wegeführung zu groß. Erfolgt zudem im untersuchten Unternehmen die Zahlungsabwicklung an Zentralkassen oder in Check-out-Zonen, so bleibt nicht einmal die Information unterschiedlicher Zeitpunkte von Käufen.

116 Vgl. Böcker, F., Die Bestimmung der Kaufverbundenheit von Produkten, a.a.0., S. 86 .

117 Vgl. Stahl, P., Verbundwirkungen im sortiment, a.a.o., S. $117 \mathrm{ff}$. 
Trotz dieser Probleme stellt die Vernachlässigung der Asymmetrie von Verbundbeziehungen eine Minderung der Aussagefähigkeit dar. Insbesondere im Hinblick auf die Ermittlung von Ursachen der Verbundbeziehungen stellt die Konzentration auf symmetrische Beziehungen eine Einschränkung dar. Die eigentliche Ermittlung von Verbundbeziehungen bleibt dagegen unberührt.

Die einzige Möglichkeit, den auslösenden und ausgelösten Kauf $z u$ bestimmen, läge in einer Befragung. Dabei ist der Konsument gezwungen, seine Handlungsweise $\mathrm{zu}$ rationalisieren. Dies ist aber vor allem bei Impulskäufen oder z.B. beim Zusammenkauf gemeinsam beworbener Artikel kaum möglich. oft bildet die Zusammenstellung von Artikeln für eine Werbung eine Vorstellung konsumentenbezogener Verbundeffekte durch den Anbieter dar. Dies führt $z u$ einem schwächeren Bewußtsein für auslösende und Folgekäufe bei den Konsumenten. Insgesamt stellt sich also auch die Befragung als problembehaftetes Mittel der Fundierung von Richtungsaspekten in Verbundbeziehungen dar.

Letztlich besteht noch die Möglichkeit, Hypothesen aufzustellen, die plausible wirkungszusammenhänge in bezug auf den auslösenden Kauf darstellen. Allerdings ist eine exakte Falsifizierung solcher Hypothesen auf der Basis empirischer Werte aus Kaufvorgängen kaum durchführbar, und Plausibilitätsannahmen alleine sind für die Fundierung einer asymmetrischen Verbundanalyse nicht ausreichend. Daher wird für diese Untersuchung ein symmetrischer Verbund zugrunde gelegt. Das bedeutet eine Anpassung an die Vorgehensweise anderer empirischer Untersuchungen zum Themenbereich Verbundanalyse. ${ }^{118}$ In Abbildung 12 werden die unterschiedlichen

118 Vgl. z.B. Böcker, F., die Bestimmung der Kaufverbundenheit von Produkten, a.a.O., S. 86; ebenfalls das symmetrische Modell unterstellen Merkle, E., Die Erfassung und Nutzung von Informationen über den Sortimentsverbund 
kausalen Beziehungen des symmetrischen und asymmetrischen Verbundes dargestellt. Dem symmetrischen Verbund wird als grundlegendes statistisches Verfahren die Korrelationsanalyse zugeordnet. Mit ihr kann gezeigt werden, wie die Käufe zweier Güter zusammenhängen. Doch erst die Anwendung multivariater Verfahren trägt dazu bei, das gesamte komplexe Geflecht von Verbundbeziehungen abzubilden.

\begin{tabular}{|c|c|c|}
\hline VERBUNDBEZIEHUNG & ASYMMETRISCH & SYMMETRISCH \\
\hline MODELL & $\begin{array}{l}\text { KAUF VON GUT-1 LÕST } \\
\text { KAUF VON GUT-2 AUS }\end{array}$ & $\begin{array}{l}\text { ZUSAMMENKAUF VON GUT-1 } \\
\text { UND GUT-2 WIRD DURCH } \\
\text { EINEN UNBEKANNTEN } \\
\text { FAKTOR X AUSGELÖST }\end{array}$ \\
\hline BEISPIEL & $\begin{aligned} \mathrm{G} 1= & \text { COMPUTER } \\
\mathrm{G} 2= & \text { SOFTWARE ODER } \\
& \text { SERVICES }\end{aligned}$ & $\begin{aligned} \mathrm{G} 1= & \text { COMPUTER } \\
\mathrm{G2}= & \text { FACHUTERATUR } \\
\mathrm{F}_{\mathrm{X}}= & \text { ANFERTIGUNG EINER } \\
& \text { WISSENSCHAFTLICHEN } \\
& \text { ARBEIT }\end{aligned}$ \\
\hline $\begin{array}{l}\text { GRUNDLEGENDES } \\
\text { STATISTISCHES } \\
\text { ANALYSEMODELL }\end{array}$ & REGRESSIONSANALYSE & KORRELATIONSANALYSE \\
\hline
\end{tabular}

Abb. 12: Symmetrischer und asymmetrischer Verbund

Darüber hinaus beeinflußt die wahl des symmetrischen Verbundmodells auch die Häufigkeitsmatrix, in der die Verbundkäufe erfaßt werden.119 Die Gleichsetzung des Kaufpaares

in Handelsbetrieben, a.a.O.; Ehrenberg, A.S.C., Repeat buying, Amsterdam 1972, S. $179 \mathrm{ff}$.

119 Vgl. Kapitel B.3. dieser Untersuchung. 
$\mathrm{y}_{1} / \mathrm{y}_{2}$ mit dem Kaufpaar $\mathrm{y}_{2} / \mathrm{y}_{1}$ führt zu einer Gleichbedeutung der Matrixzelle zeile $y_{1}$ und spalte $y_{2}$ mit der zelle Teile $y_{2}$ und Spalte $y_{1}$. Daher werden die Käufe nur in eine Hälfte der Häufigkeitsmatrix eingetragen, die andere Hälfte bleibt leer.

\subsection{Das Aggregationsniveau als Bestimmungsfaktor der Ver- bundbeziehung}

Ebenfalls ist das Aggregationsniveau, auf dem die Verbundbe$z$ iehungen erfaßt werden, $z u$ bestimmen. Dabei besteht die Möglichkeit, auf Artikel-, Abteilungs- oder Warengruppenebene vorzugehen. Die Entscheidung über das Aggregationsniveau wird vor allem durch den Umfang des Datenmaterials und die Zielsetzung der Untersuchung bestimmt. Die Zahl der möglichen Verbundbeziehungen wächst selbst bei Annahme eines symmetrischen Verbundes sehr schnell. Sie läßt sich durch die Formel

$$
\mathrm{v}=\frac{\mathrm{n} !}{2 !(\mathrm{n}-2) !}
$$

mit $V=$ Anzahl möglicher symmetrischer Verbundbeziehungen bestimmen. Dabei gibt $\mathrm{n}$ die Anzahl der Sortimentsteile an. Daraus folgt, daß sich für $n=10$ bereits 45 und für $n=300$ schon 4,5 Millionen mögliche Beziehungen ableiten lassen. Gleichzeitig muß für eine repräsentative Besetzung aller Verbundmöglichkeiten die Anzahl der untersuchten Käufe stark steigen. Insgesamt sollten nicht mehr als ca. 100 Sortimentsteile simultan analysiert werden. 120 Aus diesem Grunde wird im folgenden für die Analyse von Verbundkäufen die Abteilungsebene gewählt. Auf Artikelebene müßten in der zu-

120 Vgl. Heidel, B., Die Nutzung von Scannerdaten zur Planung der Absatzpolitik von Einzelhandelsbetrieben, a.a.o., s. 121 . 
grundeliegenden Handelsunternehmung mehr als 100.000 Sortimentsteile in die Verbundanalyse eingehen.

Darüber hinaus besteht auch aus Gründen der Interpretierbarkeit der Ergebnisse die Notwendigkeit der Aggregation auf Abteilungsebene. So ist vor dem Hintergrund der Fülle an Daten, die sich durch den Einsatz elektronischer Kassen ergeben, ein selektives und stufenweises Vorgehen zur Erfassung von Verbundbeziehungen anzustreben.121 Für einen umfassenden Überblick über Verbundbeziehungen, z.B. das Vollsortiment eines Warenhauses, bietet sich die Abbildung durch die Verbundbeziehungen zwischen Abteilungen an.

Erst in einem zweiten Schritt sollte mit Hilfe des für die übergeordnete Analyse entwickelten und eingesetzten Instrumentariums auch eine Analyse in Teilsortimenten $z \cdot B$. innerhalb der Abteilungen erfolgen. Die Anzahl der Hierarchiestufen dieser Vorgehensweise wird dabei durch den Umfang des Sortiments bestimmt. Gleichzeitig steht dem stärkeren Detaillierungsgrad der unteren Analysestufen der zunehmende Verlust des Gesamtüberblicks gegenüber. Somit ist bei der Auswahl des jeweiligen Aggregationsniveaus einer empirischen Analyse auf die zugrundeliegende Fragestellung $\mathrm{zu}$ achten.

Die zweite Aggregationsebene, die bei der Verbundanalyse von Bedeutung ist, betrifft den Aggregationsgrad der Untersuchungspersonen. Dieser wird vorwiegend durch die gewählten Vorgehensweisen der Analyse 122 festgelegt. Die Überführung der Kaufpaare in die Häufigkeitsmatrix in der ersten stufe des Kaufverbundes führt zur Aufgabe der Individualebene. Dagegen erlaubt die weitgehende Beibehaltung personenbezogener

121 Vgl. Merkle, E., Die Erfassung und Nutzung von Informationen über den Sortimentsverbund in Handelsbetrieben, a.a.O., S. 197 .

122 Vgl. Kapitel B.3. dieser Untersuchung. 
Merkmale bei der Nachfrageverbundanalyse ein flexibles Aggregationsniveau, das sich an der jeweiligen Fragestellung orientieren kann.

\subsection{Verbundmessung anhand von Absatz- oder UmsatzgröBen}

Der Kauf- bzw. Nachfrageverbund kann auch anhand des Umsatzes bzw. der Absatzmenge gemessen werden. Hierbei ist im ersten Schritt zu untersuchen, welche Ausagen und welche Probleme die beiden Möglichkeiten bieten.

Die Erfassung über Umsatzgrößen bietet gegenüber der reinen Erfassung von Absatzgrößen (Kauf bzw. Nichtkauf) den Vorteil detaillierterer Informationen. Diese sind bei der Untersuchung spezieller phänomene, z.B. der verbundbezogenen Deckungsbeitragsrechnung ${ }^{123}$ oder der Nutzung von Verbundinformationen für preispolitische Entscheidungen ${ }^{124}$ notwendig.

Für die Untersuchung eines komplexen Sortimentes bietet sich die Verbundanalyse auf der Basis von Umsatzwerten nicht an. Auf dem Aggretationsniveau Abteilung und den Daten eines Vollsortimenters wären starke Verzerrungseffekte $\mathrm{zu}$ erwarten. Unterschiedliche Abteilungen innerhalb eines Vollsortimentes können unterschiedliche Preisniveaus aufweisen. Dadurch erhalten Verbundkäufe in unterschiedlichen Abteilungen verschiedene Bedeutungen. Die Verbundkäufe gehen bei der umsatzorientierten Verbundmessung mit diesen werten in die Analyse ein. Das führt $z u$ einer Gewichtung der Ergebnisse

$123 \mathrm{Vgl}$. ebenda, S. $161 \mathrm{f}$; Sabel, H., Produktpolitik in absatzwirtschafticher Sicht, Grundlagen und Entscheidungsmodelle, Wiesbaden 1971, S. $176 \mathrm{ff}$.

124 Vgl. Simon, H., Preismanagement, wiesbaden 1982, S. 418; Diller, H., Preispolitik, 2. Aufl., stuttgart, Berlin, Köln 1991, S. 274 ff.; Diller, H., Die Wirkung von Verkäufen unter Einstandspreisen im Lebensmitteleinzelhandel, in: DBW, 41. Jg., S. $409 \mathrm{ff}$. 
zugunsten der Abteilungen mit hohem Preisniveau. Die ermittelten Verbundbeziehungen wären somit verfälscht.

Die Verwendung von Absatz- oder Umsatzgrößen hat darüber hinaus Einfluß auf das Skalenniveau der Ausgangsdaten einer Verbundanalyse. 125 Grundsätzlich liegt bei der Erfassung von Verbundgrößen anhand von Absatzzahlen eine Nominalskalierung vor, die zu Häufigkeiten aggregiert wird. Im Falle einer Erfassung von Umsatzwerten liegt dagegen Intervall-oder Verhältnisskalenniveau vor. Dies führt allerdings zu Problemen bei der Weiterverarbeitung der Daten. Die Preisdifferenzen führen bei Regressionsrechnungen $z u$ Verzerrungen, selbst wenn die Daten normiert werden. Auch der Mehrfachkauf desselben Gutes ist problematisch und kann sogar zu falschen Ergebnissen führen. 126

Insgesamt erscheint der Informationsgewinn, der sich aus der Erfassung von Umsatzdaten gewinnen läßt, eher gering und führt darüber hinaus $z u$ Verzerrungen. Zudem ist diese Methode wohl nur für eine verbundorientierte Deckungsbeitragsrechnung und eine darauf aufbauende Preispolitik von Bedeutung. Daher wird die weitere Untersuchung auf der Basis von Absatzdaten durchgeführt.

$125 \mathrm{Vgl}$. Böcker, F., Die Bestimmung der Kaufverbundenheit von Produkten, a.a.O., S. 78 ff.; Merkle, E., Die Erfassung und Nutzung von Informationen über den Sortimentsverbund in Handelsbetrieben, a.a.0., s. $38 \mathrm{ff}$.

126 Vgl. Merkle, E., Die Erfassung und Nutzung von Informationen über den Sortimentsverbund in Handelsbetrieben, a.a.o., s. $41 \mathrm{f}$. 


\section{Besonderheiten der Verbundmessung mit Kundenkarten}

\section{1 struktur und Inhalte von Rundenkarteninformationen}

Für die in den folgenden Ausführungen dargestellten Beispiele wird ein Datensatz eines filialisierten deutschen Handelsunternehmens zugrunde gelegt, der folgende struktur aufweist:

- Artikelnummer in Form einer EAN-Nummer,

- Abteilung (Nummer), in der der Artikel gekauft wurde,

- Menge (Anzahl) der gekauften Artikel,

- Betrag (Kaufsumme pro Artikel), der sich aus Artikelpreis und Menge ergibt,

- Kassennummer, die die vom Kunden benutzte Kasse anzeigt,

- Datum und

- Kaufzeitpunkt.

Über diese rein kaufbezogenen Daten hinaus, die in ähnlicher Form auch den bisherigen empirischen Analysen der Verbundwirkungen zugrunde gelegen haben, sind jedem Kauf noch weitere Daten zugeordnet:

- Die Kontonummer der Kundenkarte des jeweiligen Käufers. Diese ermöglicht auch die Identifizierung weiterer Käufe des Kundenkartenbesitzers $z u$ anderen Zeitpunkten. Dies bildet die Grundlage der Nachfrageverbunderfassung.

- Demographische Angaben über den Kunden in Form von Alter, Einkommen, Geschlecht sowie Adresse.

Diese Größen bilden die Grundlage für die Untersuchung unterschiedlicher Kundengruppen, die nach demographischen Kriterien gebildet werden. Somit wird jeder Kauf, der mit einer Kundenkarte getätigt worden ist, umfangreich erfaßt. 
Bei der Analyse ist allerdings zu berücksichtigen, daß diese Daten überwiegend die Entscheidungen eines Kundensegmentes repräsentieren, das $z u$ den umsatzstarken Kunden des betrachteten Handelsunternehmens $z u$ rechnen ist. 127 Die einzige nicht analysierbare Gruppe sind die Konsumenten unter 18 Jahren. Dies ist systemimmanent, da für diese Gruppe aus rechtlichen Gründen keine Karten ausgestellt werden. Käufe für diese Gruppe sind nur auf Basis des Gesamthaushaltes feststellbar, indem der Kartenbesitzer nicht nur Käufe für die eigene Person tätigt.

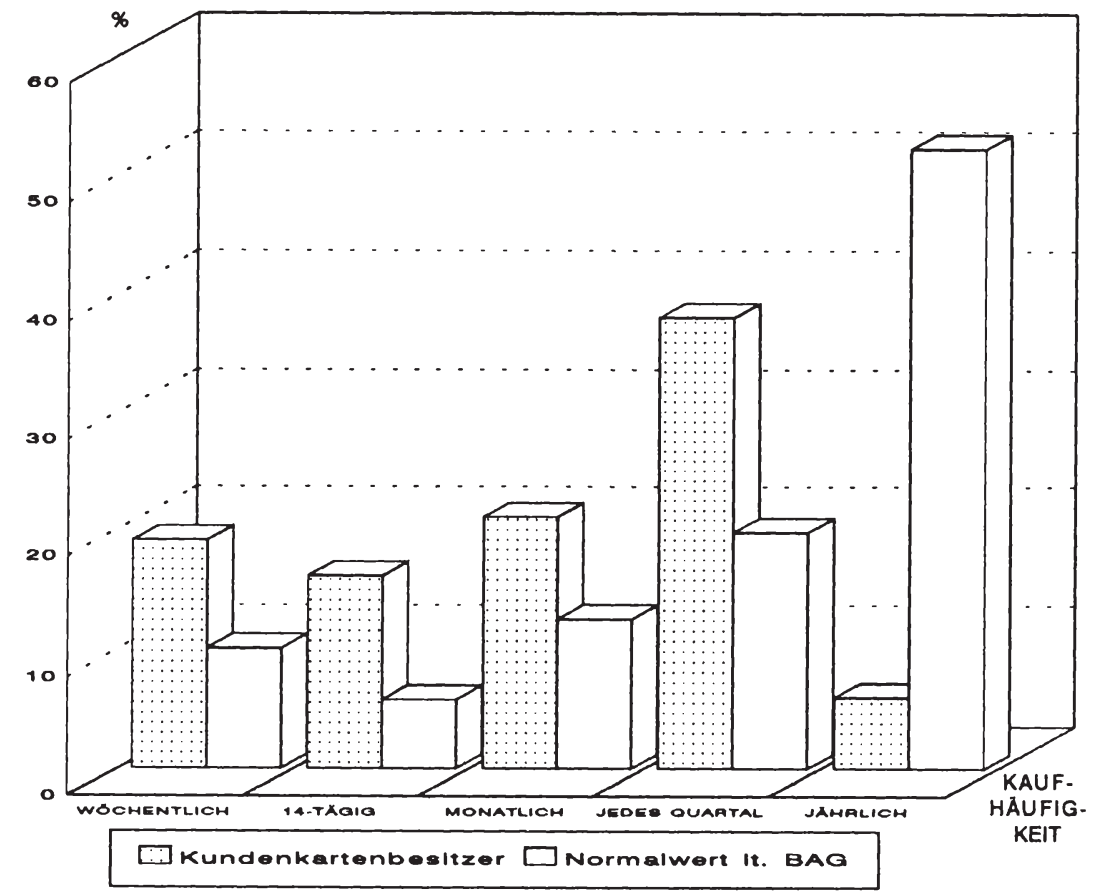

Abb. 13: Vergleich von Kaufhäufigkeiten bei Kundenkartenbesitzern und Nicht-Kartenbesitzern

127 Dies kann z.B. einem Vergleich der Analyse von Frequenzklassen zwischen der BAG-Untersuchung und den Kundenkartendaten eines Handelsunternehmens entnommen werden (Abbildung 13). 
Die Kundenkarteninformationen zeigen zwar die Käufe einer Einzelperson auf. Es ist jedoch nicht auszuschließen, daß die Käufe zur Deckung des Bedarfs eines Haushaltes mit mehreren Personen dienen. Aus diesem Grund werden im folgenden stets die Einkäufe als Käufe eines Haushalts betrachtet. Da für den Handel auch nur die Bedarfsstruktur des Gesamthaushaltes relevant ist, werden dadurch Abgrenzungsprobleme zwischen den Verbundeffekten bei Einzelpersonen und Haushalten vermieden.

Darüber hinaus besteht die Möglichkeit, daß nicht alle Käufe erfaßt werden. So kann der Kunde z.B. dann, wenn er die kundenkarte vergessen hat oder er aus anderen Gründen nicht mit der Karte bezahlen will, einzelne produkte auch bar bezahlen. Dies könnte zu Lücken bei der Untersuchung von Verbundbeziehungen führen. Doch wird dieser Restfehler vor allem aufgrund der Tatsache, daß nur gängige Artikel im sortiment des betrachteten Handelsunternehmens enthalten sind, als vernachlässigbar angesehen.

\subsection{Zielgruppenspezifische Analysemöglichkeiten aufgrund der zurechenbarkeit sozioökonomischer Informationen zu Rauf- daten}

Durch die Möglichkeit der Zuordnung der Kaufdaten eines Kunden zu den demographischen Daten, bieten sich über die bisherigen Analysemöglichkeiten im Bereich der Verbundmessung neue Ansatzpunkte. So ist es zum Beispiel möglich, alle Analysen in verschiedenen sozioökonomischen Gruppen durchzuführen. 128

$128 \mathrm{Zu}$ sozioökonomischen Kriterien bei der Marktsegmentierung vgl. Freter, H., Marktsegmentierung, stuttgart, Berlin, Köln, Mainz 1983, S. 49 ff.; dort werden Alter, Geschlecht und Einkommen als erste drei Kriterien dargestellt. 
Im aktuellen Datensatz sind Alter, Einkommen und Geschlecht angegeben. Daher bieten sich diese Kriterien zur Bildung von Untersuchungsgruppen an. Sie besitzen auch eine stark differenzierende Wirkung zwischen Personengruppen.129 Grundsätzlich besteht jedoch die Möglichkeit, auch andere Merkmale eines Kundenkartenbesitzers zu erfassen, z.B. Geschlecht, Familienstand und Bildungsniveau.

Nach Durchführung von Analysen in spezifischen Gruppen, in denen zielgruppenbezogene Fragestellungen beantwortet werden, besteht eine weitere Analysemöglichkeit im Vergleich der Ergebnisse der Verbundanalysen. Ergeben sich bei einem Vergleich starke Divergenzen bzgl. der Verbundbeziehungen zwischen den Gruppen, so ist deutlich, daß die für die Untersuchungsgruppenbildung verwendeten Kriterien Gruppen mit verschiedenen Verbundkonstellationen eindeutig abgrenzen.

Aus den unterschiedlichen Verbundkonstellationen sind dann Maßnahmen für die Bearbeitung der einzelnen Zielgruppen ableitbar. So z.B. ist für die zusammenstellung von Werbeware für ein Direkt-Mailing an eine junge zielgruppe mit mittlerem Einkommen die Konstellation der Verbundbeziehungen innerhalb der entsprechenden Untersuchungsgruppe von besonderer Bedeutung.

Ein weiterer Wirkungsaspekt der Zurechnung sozioökonomischer Informationen $z u$ Kaufdaten betrifft die Untersuchung von Zielgruppen, die nach Verbundkaufinformationen gebildet werden. 130 Dabei dienen sie als beschreibende Kriterien. Darüber hinaus kann anhand des Homogenitätsgrades der Merkmale innerhalb der zielgruppen deren Eignung zur segmentierung überprüft werden.

$129 \mathrm{Vgl}$. Freter, H., Marktsegmentierung, s. 50 f.

$130 \mathrm{Vgl}$. Kapitel B.3.33 dieser Untersuchung. 


\subsection{Mehrfachmessung von Kaufverbunden gleichbleibender Kun- dengruppen auf Basis der Identifizierbarkeit eines Kun- den $z u$ verschiedenen Raufzeitpunkten}

Jeder Kauf muß einem speziellen Kartenkonto des Kundenkarteninhabers zugerechnet werden, so daß in jedem Kaufdatensatz auch die Kundenkartennummer mit gespeichert ist. Diese Tatsache ermöglicht es, den Kunden bei späteren Käufen wieder zu identifizieren bzw. Kundenkartenkäufe zu unterschiedlichen Kaufzeitpunkten demselben Kunden zuzuordnen. Dadurch ergibt sich eine Ausweitung der zeitpunktbezogenen sicht des Kaufverbundes. Die Verfolgung eines Kunden über einen längeren Zeitraum ermöglicht somit die Erfassung einer kompletten individuellen Kaufstruktur. Diese Zuordnungsmöglichkeit eröffnet, ähnlich wie die Zuordnung der sozioökonomischen Informationen, weitergehende Möglichkeiten der Verbundanalyse, als dies bei bisherigen empirischen Untersuchungen möglich war.

Als erste Anwendung einer derartig zeitraumbezogenen Analysemöglichkeit auf das bisherige zeitpunktbezogene Konzept des Kaufverbundes ergibt sich die Mehrfachmessung von Kaufverbunden. So war zwar bisher die Möglichkeit gegeben, zu unterschiedlichen zeitpunkten Verbundbeziehungen zu erheben, doch konnte für die Erhebung keine konstante Gruppe von Kunden vorausgesetzt werden. Die durch die Kundenkarten eröffneten Möglichkeiten der Mehrfachmessung kann vor allem der Validierung der erhaltenen Ergebnisse dienen.

Darüber hinaus kann durch die Identifizierung spezieller Kunden eine Untersuchungsgruppe gebildet werden, die zu verschiedenen zeitpunkten analysiert werden kann. Dadurch können, wenn die untersuchten zeitpunkte relativ gleiche Umfeldbedingungen (z.B. Adventszeit) aufweisen, strukturveränderungen im Verbundkaufverhalten festgestellt werden. Daraus 
können wiederum Implikationen z.B. für das Data-Base-Marketing abgeleitet werden.

Sind die in der Untersuchungsgruppe zusammengefaßten Kunden ferner nach sozioökonomischen oder weitergehenden Kriterien vorselektiert, lassen sich durch einen Vergleich der Verbundkonstellationen $z u$ verschiedenen zeitpunkten wie bei einer Kohortenanalyse Entwicklungstendenzen ableiten. ${ }^{131} \mathrm{Da}$ die Gruppen in sich homogen sind, können die Ergebnisse verwendet werden, um Maßnahmen für Kundengruppen in einem früheren Entwicklungsstand abzuleiten. 132

131 In Kohortenanalysen werden Gruppen von Konsumenten im Zeitablauf verfolgt. Da die Gruppenmitglieder durch gemeinsame Merkmale gekennzeichnet sind, wird ihrer Entwicklung eine hohe prognostische Relevanz für das Verhalten anderer Konsumenten zugesprochen, die die gleichen Merkmale $z u$ einem späteren zeitpunkt aufweisen. Vgl. zur Kohortenanalyse Hüttner, M., Die Kohortenanalyse als Instrument der strategischen Marktforschung, in: Realisierung des Marketing, Belz, C. (Hrsg.), Bd.1, St. Gallen 1986, S. 309 ff.; Rentz, J.O., Reynolds, F.D., stout, R.G., Analyzing Changing Consumption Patterns with Cohort Analysis, in: Journal of Marketing Research, Vol. 7, Febr. 1983, S. 12 ff.; Reynolds, F.D., Rentz, J.O., Cohort Analysis: An Aid to strategic Planning, in: Journal of Marketing, Vol. 45, Summer 1981, S. $62 \mathrm{ff}$.

132 Weil für den Aufbau einer solchen umfassenden Analyse ebenso umfangreiche Daten wie auch lange Zeitreihen benötigt werden, und diese Voraussetzungen für den vorliegenden Datensatz bisher nicht gegeben sind, wird dieser Untersuchungsansatz im folgenden jedoch ausgeklammert. 
3.4 Die Erfassung von Rauf- und Nachfrageverbund auf der Basis von Kundenkarteninformationen

\subsection{Der Kaufverbund als zeitpunktbezogener Ansatz der ver- bundmessung}

Der Kaufverbund stellt sich - wie aus seiner Definition ersichtlich - als zeitpunktbezogenes Konstrukt dar, das die Frage untersucht, was von einem Kunden zusammen gekauft wird. Dabei ist es nicht von Interesse, welcher Kunde etwas zusammen gekauft hat, sondern ausschließlich, was zusammen gekauft wurde. Die demographischen Daten eines Kunden können dabei zur Bildung von Untersuchungsgruppen herangezogen werden. Oberstes ordnungskriterium bleibt allerdings stets der Zeitpunkt eines Kaufs.

Vor diesem Hintergrund ist ein allgemeines (empirisches) Vorgehen abzuleiten, das in Abbildung 14 dargestellt ist. Im ersten Schritt ist dabei festzulegen, auf welchem Aggregationsniveau der Kaufverbund zu analysieren ist. Für das Beispiel und auch die spätere empirische Analyse wird die Abteilung als Aggregationsniveau gewählt. Im folgenden werden dann die einzelnen Kaufdatensätze $\mathrm{KDS}_{i}$ zunächst für die endgültige Verarbeitung im Rahmen einer clusteranalyse aufbereitet.

Nach einer Überprüfung der Gruppenzugehörigkeit des Kunden, von dem der Kaufdatensatz stammt, erfolgt eine Prüfung der Anzahl besuchter Abteilungen. Kaufdatensätze, die nur eine besuchte Abteilung enthalten, werden zur Analyse nicht herangezogen, da durch einen solchen Kauf keine Verbundbeziehungen abgebildet werden können. 133

$133 \mathrm{Vgl}$. Kapitel B.2.22 dieser Untersuchung.

Marcus Poggenpohl - 978-3-631-75099-5 


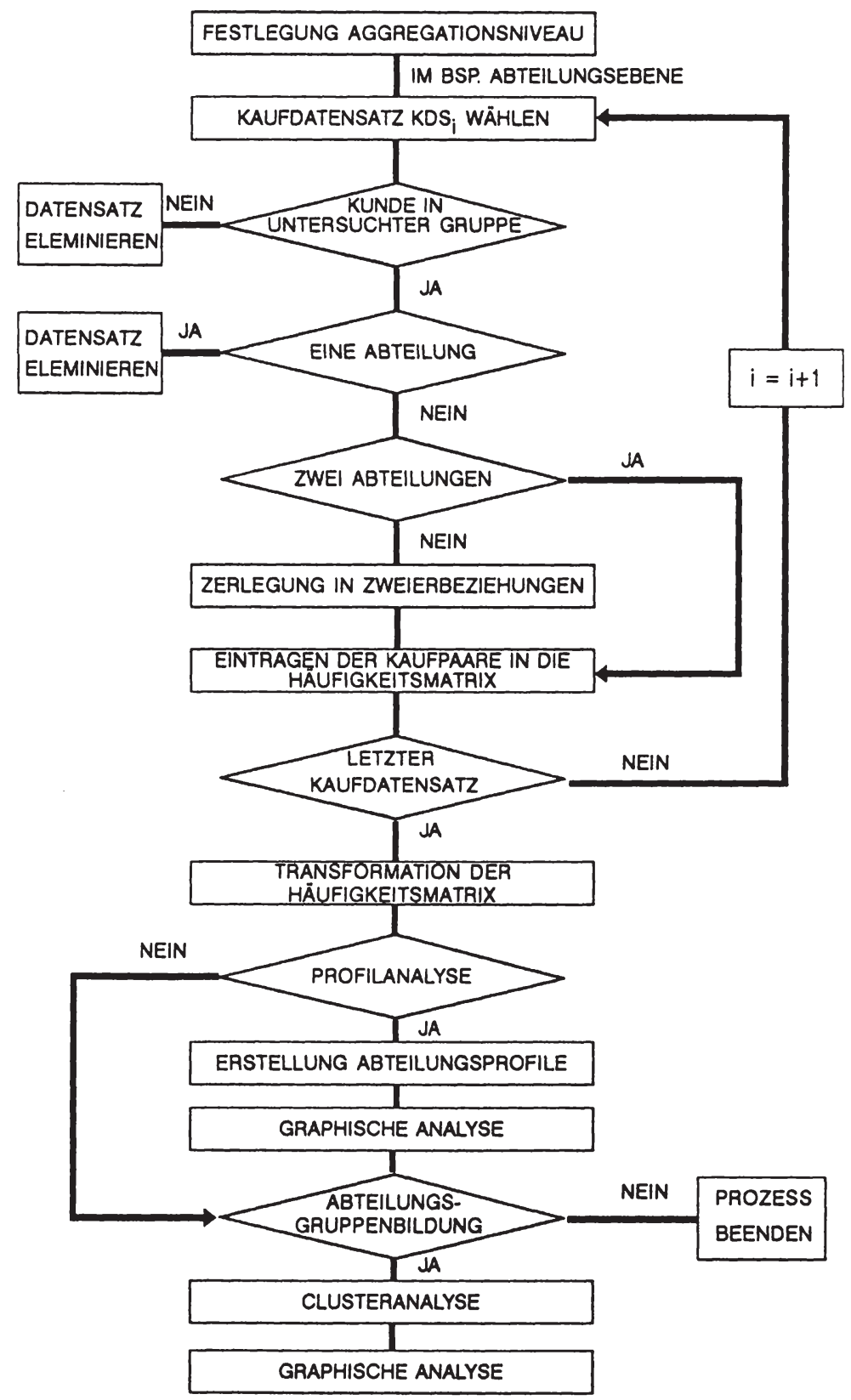

Abb. 14: Ablaufdiagramm Kaufverbundanalyse 
Enthält der Kaufdatensatz zwei Abteilungen, so kann dieses Kaufpaar direkt in die Häufigkeitsmatrix für Verbundkäufe eingetragen werden (vgl. Abbildung 15). Wenn z.B. die Abteilungen $A$ und $B$ mit Käufen registriert werden, so ergibt sich ein Eintrag in die Häufigkeitsmatrix in zeile 1 und Spalte 2 mit dem Wert 1 . Ergeben sich weitere Fälle der gleichen Kombination, so werden die Werte aufsummiert.

\begin{tabular}{c|c|c|c|c|c|c|c|c|} 
& $A B T$ A & ABT B & $A B T$ C & $A B T$ D & ABT E & ABT F & $\ldots \ldots \ldots . .$. & ABT Z \\
\hline ABT A & - & 1 & & & & & & \\
\hline ABT B & - & - & & & & & & \\
\hline ABT C & - & - & - & & & & & \\
\hline ABT D & - & - & - & - & & & & \\
\hline ABT E & - & - & - & - & - & & & \\
\hline ABT F & - & - & - & - & - & - & & \\
\hline$\vdots$ & & & & & & & & \\
$\vdots \vdots$ & & & & & & & & \\
\hline ABT Z & - & - & - & - & - & - & & \\
\hline
\end{tabular}

Abb. 15: Häufigkeitsmatrix von Verbundkäufen

Enthält der Kaufdatensatz mehr als zwei Abteilungen, so ist eine Zerlegung in mehrere Kaufpaare notwendig. Ein Kaufdatensatz, der die Abteilungen $A, B$ und $C$ enthält, ist somit in die Kaufpaare $A B, A C$ und $B C z u$ erlegen und in die Häufigkeitsmatrix $z u$ überführen. Dabei wird in der Literatur häufig gefordert, daß die Kaufpaare aus einem Kauf mit mehr als zwei Abteilungen mit dem Faktor 


$$
d=\frac{1}{m-1}
$$

gewichtet in die Häufigkeitsmatrix überführt werden.134 Dabei steht $m$ für die Anzahl der im Kaufdatensatz enthaltenen Abteilungen.

Als Begründung für dieses Vorgehen wird angeführt, daß dadurch gewährleistet wird, daß die spalten und zeilennummern der Matrix die Anzahl der Kaufakte widerspiegeln ${ }^{135}$ oder daß die einzelne Abteilung, die in einem Kaufakt mit vielen anderen Abteilungen steht, zu stark gewichtet wird. Das ergibt sich, da die Zahl der Zweierbeziehungen mit steigender Anzahl von Abteilungen in einem Kaufakt überproportional nach der Formel

$$
\mathrm{z}_{\mathrm{KP}}=\frac{\mathrm{m}(\mathrm{m}-1)}{2}
$$

ansteigt, wobei $\mathrm{z}_{\mathrm{KP}}$ die Anzahl $\mathrm{zweierbeziehungen} \mathrm{und} \mathrm{m}$ die Anzahl der im Kaufdatensatz enthaltenen Abteilungen darstellt.

Im Rahmen der Kaufverbundanalyse ist dieses Vorgehen jedoch nicht geeignet. Die Matrix enthält nicht die Anzahl der Kaufakte, sondern die der Verbundbeziehungen. Dieser Aspekt widerlegt auch das zweite Argument, da durch das überproportionale Ansteigen nicht etwa eine Abteilung überbewertet,

134 Vgl. Hruschka, H., Die Bestimmung der Kaufverbundenheit mit Hilfe eines probabilistischen Meßmodells, a.a.0., s. 418; Merkle, E., Die Erfassung und Nutzung von Informationen über den Sortimentsverbund in Handelsbetrieben, a.a.o., s. 50 .

$135 \mathrm{Vgl}$. Hruschka, M., Die Bestimmung der Kaufverbundenheit mit Hilfe eines probabilistischen Meßmodells, a.a.0., s. 418 . 
sondern die Anzahl ihrer Verbundbeziehungen exakt abgebildet wird. Eine Abteilung im Zusammenkauf mit vielen anderen Abteilungen weist grundsätzlich mehr Kaufverbundbeziehungen auf als im Zusammenkauf mit nur einer Abteilung.

Das wichtigste Argument ist jedoch das Fehlen einer Begründung für eine stärkere Gewichtung eines Kaufpaares aus einem Kaufakt mit wenigen Abteilungen, z.B. wird einem Kaufpaar aus einem Kaufakt mit zwei Produkten der Wert 1 zugeordnet. Ein Kaufpaar aus einem Kaufdatensatz mit z.B. drei Abteilungen geht mit dem wert

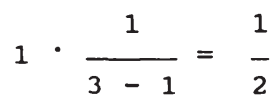

in die Häufigskeitsmatrix ein. Das bedeutet eine Überbewertung von Kaufakten, die wenige Produkte umfassen. Zudem erhöht sich diese Diskrepanz bei steigender Anzahl von Abteilungen in einem Datensatz. Aus diesem Grund werden alle Verbundbeziehungen mit dem Wert 1 in die Matrix überführt. Die Verbundbeziehungen sind symmetrisch. Deshalb muß nur eine Hälfte der Matrix gefüllt werden.

Obgleich aufgrund der skizzierten Vorgehensweise alle Beziehungen zwischen Käufen abgebildet werden, entsteht bei Kaufakten mit mehr als zwei produkten ein gewisser Informationsverlust. Zwar gewährt die Zerlegung in Zweierpaare die Einbeziehung aller Verbundbeziehungen zwischen jeweils zwei Artikeln. Für die überwiegende Zahl von Beziehungen ist das ausreichend. Problematisch ist es erst bei einer "Verbundbeziehung höherer Ordnung". Dieser Ausdruck bezeichnet einen Kauf z.B. von drei Produkten, die erst bei gemeinsamem Erwerb nutzbringend für den Konsumenten sind. Dabei steht dann ein gemeinsamer Faktor hinter dem Kauf aller drei Produkte. Die Hinweise auf eine derartige Verbundkonstruktion gehen bei der zerlegung in zweierbeziehungen verloren. 
Insgesamt sind solche dezidierten Hintergrundaspekte bei Mehrproduktkäufen eher selten, so daß im Rahmen dieser Untersuchung das skizzierte Vorgehen weiter verfolgt wird.

Für den Einsatz einer Clusteranalyse zur Bildung von Abteilungsgruppen ist die Matrix in einem weiteren schritt zu transformieren. ${ }^{136}$ Dann wird sie als Distanz- bzw. Ähnlichkeitsmatrix in eine clusteranalyse überführt. Die dabei entstehenden cluster können darauf aufbauend noch im Rahmen einer Diskriminanzanalyse auf ihre Güte hin überprüft werden.

Eine Alternative besteht in einem Vorgehen, das Merkle als Profilanalyse bezeichnet. ${ }^{137}$ Dazu wird zunächst jeder einzelnen Abteilung die Zusammenkaufhäufigkeit mit jeder anderen Warengruppe zugeordnet. Dazu wird die Häufigkeitsmatrix in transformiertem oder auch nicht transformiertem zustand zeilenweise ausgelesen. Bei einer graphischen Umsetzung ergeben sich somit für jede Abteilung unterschiedliche verbundkaufprofile, die auf einen Blick aufzeigen, wo starke und wo schwache Verbundbeziehungen vorliegen (vgl. Abbildung 16). Legt man im Rahmen einer graphischen Analyse mehrere Profile übereinander, so lassen sich Häufungen starker Verbunde erkennen.

Jedoch wird dieses Verfahren bei steigender zahl untersuchter Warengruppen schnell unübersichtlich und führt auch nicht direkt zu Verbundgruppen. $\mathrm{zu}$ diesem $\mathrm{zweck}$ ist es notwendig, die Abteilungsprofile in eine clusteranalyse zu

$136 \mathrm{Vgl}$. Kapitel B.1.23 dieser Untersuchung.

$137 \mathrm{Vgl}$. Merkle, E., Die Erfassung und Nutzung von Informationen über den Sortimentsverbund in Handelsbetrieben, a.a.0., S. $108 \mathrm{ff}$. 
überführen, um Abteilungsgruppen $z u$ erhalten. 138 Auch bei diesem Verfahren bildet eine Diskriminanzanalyse den Abschluß des empirischen Vorgehens.

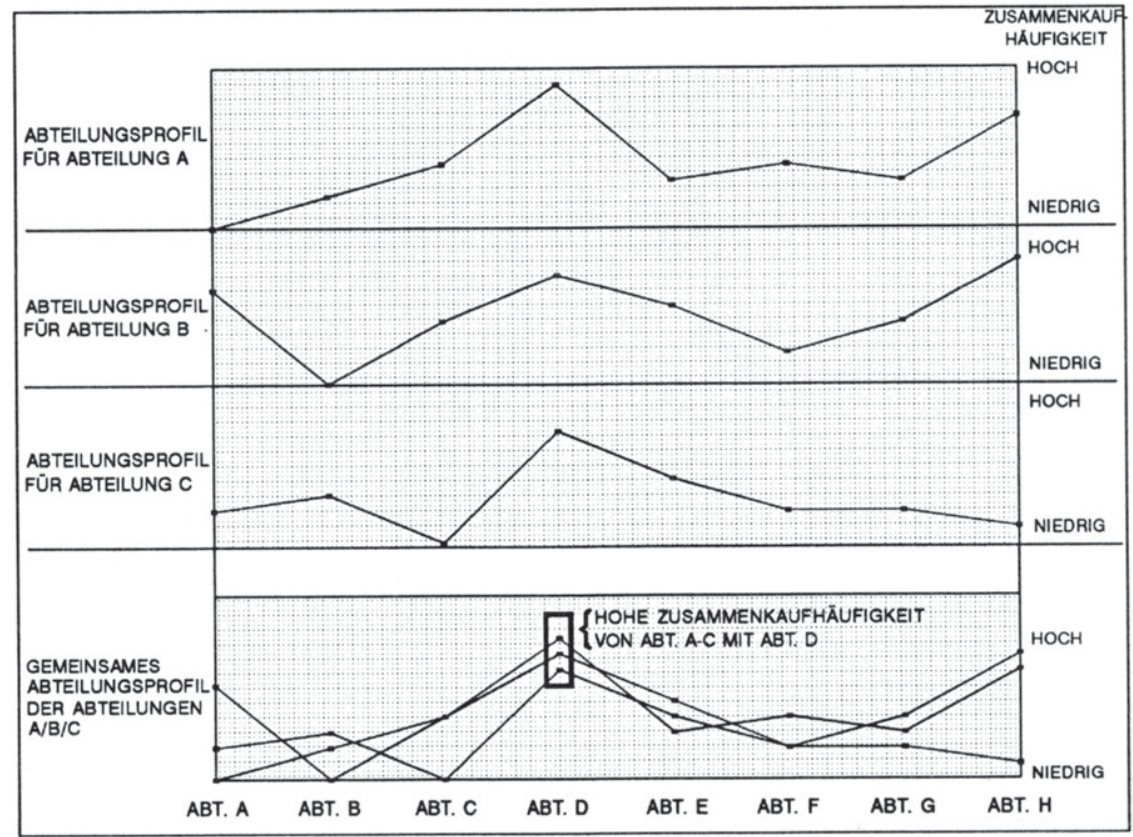

Abb. 16: Abteilungsprofile der Abteilungen A, B, C für die Abteilungen $\mathrm{A}$ bis $\mathrm{H}$

\subsection{Der Nachfrageverbund als zeitraumbezogener Ansatz der verbundmessung}

Der Nachfrageverbund unterscheidet sich schon in seiner Konzeption vom Kaufverbund. Für eine Erfassung des Nachfrageverbundes muß die gesamte Betrachtungsebene gewechselt wer-

138 Vgl. Merkle, E., Die Erfassung und Nutzung von Informationen über den Sortimentsverbund in Handelsbetrieben, a.a.0., S. $112 \mathrm{ff}$. 
den. Dabei entsteht ein zeitraum- statt eines zeitpunktbezogenen Konstruktes. Diese Tatsache hat erhebliche Auswirkungen auch auf das empirische Vorgehen.

Der zusammenführende Faktor zur Entstehung eines Verbundes ist somit nicht mehr die zeit, sondern der jeweilige Kunde. Es steht nicht mehr im Vordergrund der Betrachtung, was zusammen gekauft wird, sondern was ein Kunde kauft und welche Kaufstrukturen sich bei aggregierter Betrachtung aller Kunden ableiten lassen. Das bedeutet eine Ausrichtung der Analyse in Richtung des Bedarfsverbundes.

Diese konzeptionelle Änderung des Verbundes bewirkt, daß ein Vorgehen über von vornherein ohne Kundenbezug aggregierte Häufigkeitsmatrixen nicht möglich ist. Das empirische Vorgehen bei der Erfassung bzw. Messung ergibt sich wie in Abbildung 17 dargestellt.

Im ersten Schritt ist auch hier das Aggregationsniveau festzulegen, wobei für das Beispiel die Abteilungsebene gewählt wird. 139 Nach einer Überprüfung der Zugehörigkeit des untersuchten Kundendatensatzes zur untersuchten Gruppe, werden die Käufe in einen für jeden Kunden individuell erstellten Käuferdatensatz überführt. Dabei wird jede mögliche Kaufabteilung als Variable betrachtet, deren Ausprägung durch die Kaufhäufigkeit gegeben ist.

Aus diesen individuellen Datensätzen lassen sich wiederum direkt individuelle Käuferprofile ableiten. Diese können wie

139 Ein Vorgehen auf Artikelebene erscheint insbesondere beim Nachfrageverbund problematisch, da auf einem längeren Betrachtungszeitraum Artikel mit hohem substitutionsgrad bzw. nahezu identische produkte unterschiedlicher Marken zum großen Teil zusammengefaßt werden sollten, da sie im Rahmen des Bedarfsverbundes identische Funktionen erfüllen. Dazu sind aber aufwendige Plausibilitätsüberlegungen notwendig, die eventuell eine objektive Erfassung beeinflussen können. 


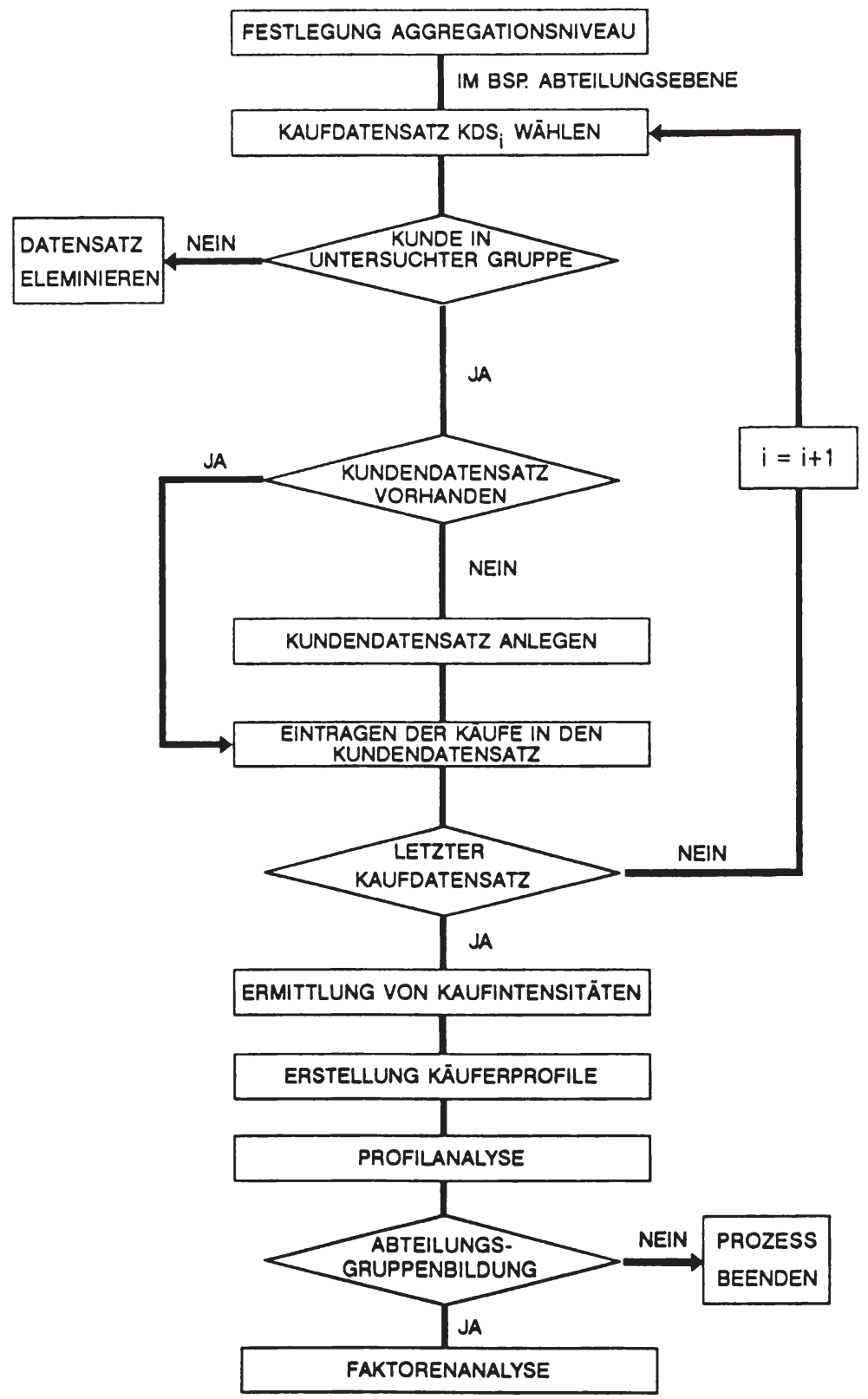

Abb. 17: Ablaufdiagramm Nachfrageverbundanalyse

Marcus Poggenpohl - 978-3-631-75099-5

Downloaded from PubFactory at 01/11/2019 08:22:38AM 
beim Kaufverbund im Rahmen einer graphischen Analyse ausgewertet werden. Der Unterschied besteht allerdings darin, daß bei der Kaufverbundanalyse ein Profil pro Warengruppe über alle Kunden und bei der Nachfrageverbundanalyse ein Profil pro Kunde erstellt wird.

Für eine Abteilungsgruppenbildung ist jedoch ein anderes Vorgehen zu wählen. Nach einer Normierung bzw. Standardisierung der Variablen können Verbundeffekte anhand einer Faktorenanalyse überprüft werden. Die entstehenden Faktoren spiegeln dann Verbundkaufgruppen wider, die durch die am höchsten auf sie ladenden Variablen bzw. Abteilungen zu beschreiben sind. Es ergibt sich somit ein sehr komplexes Bild von Verbundstrukturen.

Die erstellten Häufigkeitsprofile können jedoch nicht direkt in eine Faktorenanalyse überführt werden. In der Faktorenanalyse werden gleichartig reagierende variablen zusammengefaßt. Die Variablen im untersuchten Fall sind Artikel oder Abteilungen sowie die Reaktionen der erfaßten Kaufhäufigkeiten. Wenn Gruppierungen ausschließlich nach absoluten Häufigkeiten durchgeführt werden, so lassen sich sinnlose $\mathrm{zu}-$ sammenführungen kaum vermeiden.

So kann zum Beispiel folgende Kombination vorliegen: Ein Kunde kauft in durchschnittlichem Maße Lebensmittel mit seiner Karte, ist aber ein Intensivkäufer in der Gruppe Schallplatten/Tonträger. Da die Lebensmittelabteilung eine wesentlich größere Grundfrequenz aufweist, könnten beide Abteilungskaufhäufigkeiten gleich hoch sein. Daher würden in diesem Fall diese Abteilungen in einem Faktor zusammengeführt. Auf diese Weise ergeben sich nicht sinnvolle Verbundgruppen.

Um diesem Problem $z u$ begegnen ist es notwendig, einen neuen Faktor einzuführen, der die Kaufhäufigkeiten transformiert und in Kaufintensitätsmaße umwandelt. Dieses Maß sollte 
einen Mittelwert von 1 aufweisen, der eine durchschnittliche Kaufintensität anzeigt. Um das Maß auf jede untersuchte Stichprobe anwenden $\mathrm{zu}$ können, wird es zunächst allgemein dargestellt. Das Transformationsergebnis wird als durchschnittliche relative Kaufintensität dargestellt und mit $I_{R}$ bezeichnet:

$$
I_{R}=\frac{K_{i j}}{K_{j} \cdot i^{*}}
$$

wobei:

$\mathrm{K}_{\mathrm{ij}}=$ die Anzahl aller Käufe der Kundengruppe $i$ in der Warengruppe $j$ ist

$K_{j}=$ die durchschnittliche Anzahl der Käufe eines Kunden in der Warengruppe $j$ (= Anzahl aller Käufe aller Kunden in Warengruppe $j$ dividiert durch die Anzahl aller Kunden)

$i^{*}=$ die Anzahl der untersuchten Kunden in der Kundengruppe i

Da im Normalfall diese Transformation für jeden Kundendatensatz bzw. für jedes Kaufprofil getrennt durchgeführt wird, kann diese Formel vereinfacht werden. Zudem stellt sich die durchschnittliche relative Kaufintensität einer Kundengruppe dann als spezifische relative Kaufintensität eines einzelnen Kunden dar. Die Formel lautet dann:

$$
I_{r}=\frac{K_{a j}}{K_{j}}
$$

wobei

$\mathrm{K}_{\mathrm{aj}}=$ die Anzahl aller Käufe des Kunden a in Warengruppe $j$ $\mathrm{K}_{\mathrm{j}}=\begin{aligned} & \text { die durchschnittliche Anzahl der Käufe eines Kunden } \\ & \text { in der Warengruppe } j \text { ist. }\end{aligned}$

Die auf diese Weise transformierten Häufigkeitswerte bilden ein geeignetes Ausgangsmaterial für die Faktorenanalyse. 
Im Gegensatz zur Kaufverbundanalyse, die alle Abteilungen durch Clusteranalysen gruppiert hat, muß bei der Nachfrageverbundanalyse nicht jede Abteilung zur Gruppierung verwendet werden, wenn sie z.B. nicht oder nur schwach auf jeden Faktor lädt.

\subsection{Zielgruppenbildung anhand von verbundinformationen}

Über die Strukturanalysen und Abteilungsgruppenbildungen der Kauf- und Nachfrageverbundanalyse hinaus können die verbundinformationen auch weitergehend genutzt werden. Mit ihrer Hilfe ist es möglich, Zielgruppen nach ihrem Verbundkaufverhalten zu bilden. 140 Dabei bildet der Kaufverbund allerdings kaum eine geeignete Grundlage, da er weitaus stärker als der Nachfrageverbund von situativen Komponenten abhängig ist. Darüber hinaus gehen im Rahmen der empirischen Analyse die kundenspezifischen Informationen weitgehend bei der Erstellung der Häufigkeitsmatrix verloren.

Im Gegensatz dazu bietet der Nachfrageverbund ideale Voraussetzungen für eine zielgruppenanalyse. Zum einen bietet die Zeitraumbetrachtung größere stabilität gegenüber situativen Einflußgrößen. Zum anderen bildet der Nachfrageverbund durch seine Annäherung an den Bedarfsverbund einen Großteil der realisierten Nachfragestruktur eines Haushalts bzw. eines Individuums ab.

Darüber hinaus bietet das empirische Vorgehen zur Erfassung des Nachfrageverbundes Ansatzpunkte für eine Zielgruppenbil-

$140 \mathrm{Vgl}$. zu Kriterien und Möglichkeiten der Marktsegmentierung Freter, H., Marktsegmentierung, a.a.O., S. 44 ff.; Steffenhagen, H., Marketing - Eine Einführung, stuttgart, Berlin, Köln, Mainz 1988, S. 52 f.; Meffert, H., Marketing, a.a.o., s. 245 f. 
dung. Durch die Erfassung eines Datensatzes für jeden Kunden gehen keine kundenspezifischen Daten verloren. Im Rahmen der Kaufverbundanalyse wurde die Möglichkeit aufgezeigt, aus einzelnen Abteilungsprofilen mit $\mathrm{Hilfe}$ einer Clusteranalyse Abteilungsgruppen abzuleiten. Ein analoges Vorgehen ist mit den individuellen Käuferprofilen ebenfalls möglich. Das Ergebnis besteht jedoch nicht in Abteilungsgruppen, sondern in Kundengruppen.

Das empirische Vorgehen (vgl. Abbildung 18) lehnt sich somit weitgehend an das Vorgehen im Rahmen der Nachfrageverbundanalyse an. Von den ersten Schritten entfällt lediglich die Überprüfung der Gruppenzugehörigkeit, da die zielgruppenbildung über alle vorhandenen Kaufdatensätze durchgeführt wird. Im weiteren Verlauf entfällt die profilanalyse, die nur als graphisches Hilfsinstrument bei der Kauf- und Nachfrageverbundanalyse genutzt werden kann.

Im Gegensatz zur Nachfrageverbundanalyse werden auch die sozioökonomischen Daten des Kunden den jeweiligen individuellen Käuferprofilen zugeordnet. Sie dienen dazu, die durch die clusterung entstandenen Käufergruppen $z u$ beschreiben und zu interpretieren. 141 Ergeben sich dabei weitgehend homogene strukturen, so ist rückzuschließen, daß bestimmte Kombinationen von Ausprägungen dieser Merkmale bestimmten Nachfrageverbundstrukturen zugeordnet werden können und umgekehrt. Dies kann die Zusammenstellung von Angebotsware in nach sozioökonomischen Kriterien gestalteten zielgruppenspezifischen Werbemaßnahmen erleichtern. Ergeben sich diesbezüglich allerdings keine Homogenitäten der cluster, so ist festzustellen, daß zumindest im Hinblick auf den Nachfrageverbund sozioökonomische zielgruppen nicht zur segmentierung geeignet sind. Dann ist zu überprüfen, ob die zielgruppenbil-

141 Vgl. Freter. H., Marktsegmentierung, a.a.O., S. 87. Marcus Poggenpohl - 978-3-631-75099-5 


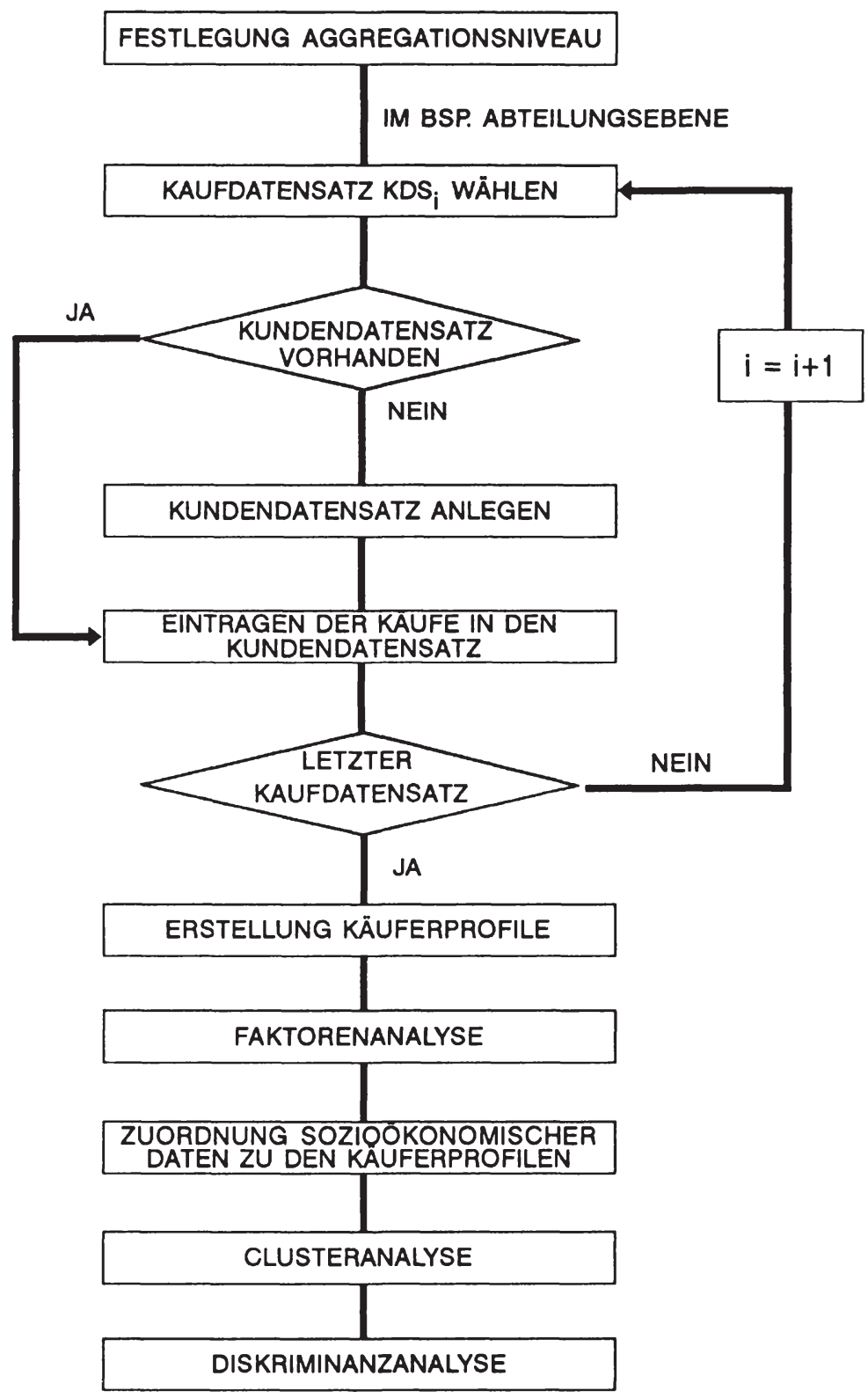

Abb. 18: Ablaufdiagramm Ziel-gruppenbildung 978-3-631-75099-5 
dung anhand der Verbundinformationen nicht zu besseren Ergebnissen für das Handelsunternehmen führt.

Da die Zielgruppenbildung anhand von Verbundinformationen auf dem tatsächlichen Kaufverhalten beruht, ist ihre Aussagekraft wahrscheinlich höher einzuschätzen als eine Gruppierung anhand personenspezifischer Faktoren. 142 Dieser Effekt wird noch dadurch verstärkt, daß die Käufe eines Kunden mit der Kundenkarte die Käufe eines gesamten Haushalts mit individuellen Daten repräsentieren kann. In diesem Fall ist die sozioökonomische zielgruppenbildung meist - wenn nicht sehr umfangreiche Daten vorliegen - unzureichend. Aber selbst bei umfangreichem Datenmaterial können nicht alle kaufbeeinflussenden Faktoren erfaßt werden, so daß grundsätzlich das tatsächliche Kaufverhalten aussagekräftiger ist.

142 Vgl. Freter, H., Marktsegmentierung, a.a.O., S. 97. 


\section{c. Empirische Analysen des Rauf- und Nachfrageverbundes eines Einzelhandelsunternehmens}

\section{Design der Untersuchung}

\subsection{Kennzeichnung der stichprobe und struktur der Daten}

Für die empirische Untersuchung von Kauf- und Nachfrageverbunden steht ein umfangreiches Datenmaterial zur Verfügung. Die Daten repräsentieren alle Käufe von Kundenkartenbesitzern eines Vollsortimenters. Die Erfassung der einzelnen Käufe erfolgte mittels Scannerkassen im Rahmen eines Warenwirtschaftssystems, so $d a \beta$ automatisch $z u$ den Kaufbeträgen auch Datum und Kundennummer erfaßt werden.

Für diese Analyse sind die Daten auf Abteilungsebene aggregiert. Dabei wurden 66 verschiedene Abteilungen erfaßt. Der Erhebungszeitraum umfaßt ein komplettes Kalenderjahr, so daß auch alle saisonalen Komponenten mit erfaßt werden, z.B. winter- und Sommerschlußverkauf oder die Weihnachtszeit, und sich ein umfassendes Bild der Verbundbeziehungen herstellen läßt. Einzelne spezifische saisonale Abschnitte werden innerhalb dieser Untersuchung jedoch nicht gesondert betrachtet, da dies den Rahmen der empirischen Arbeit überschreiten würde.

Die zur Verfügung stehenden Daten umfassen folgende Bestandteile:

- die Rundennummer des betrachteten Kaufs, die im Zeitablauf ein wiederauffinden des gleichen Kunden erlaubt,

- die Nummer der Abteilung, in der der Kauf stattfand,

- das Datum des Kaufs, den Raufzeitpunkt,

- die Kaufsumme, die in der Abteilung bezahlt wurde,

- die Anzahl der Artikel, die in der Abteilung gekauft wurden, 
- demographische Angaben und zwar

o Alter,

- Geschlecht und

- Einkommen des Kunden.

Jede weitere Angabe, vor allem Namen- und Adressdaten wurde bewußt aus dem Datensatz entfernt.

Die Minimalanforderung für die Aufnahme einer Kundennummer in den Datensatz bestand darin, daß der Kunde zumindest 2 Käufe in den Abteilungen des Handelsunternehmens innerhalb des Untersuchungszeitraumes getätigt haben mußte, damit überhaupt eine Verbundbeziehung entstehen kann.

Nach Beachtung dieser Vorgaben ergaben sich die folgenden Kennzahlen für den Datensatz:

- Betrachtet werden die Käufe von insgesamt 4.145 Kunden des Handelsunternehmens.

- Diesen Kunden werden 171.782 Raufvorgänge in 66 Abteilungen zugeordnet.

- Jedem dieser Kaufvorgänge kann eine durchschnittliche Kaufsumme von 43,95 DM bei einer Anzahl von 6,7 Artikeln pro betrachtetem Abteilungskauf zugeordnet werden, so da $\beta$ insgesamt ein Raufvolumen von 7.550.653 DM auf Verbundwirkungen analysiert werden kann.

Die 4.145 Kunden können wie folgt näher charakterisiert werden:

- 37,2 der Kunden führen zwischen 2 und 12 Kaufvorgänge pro Jahr im untersuchten Unternehmen durch, 39,2 \% weisen sogar mehr als 52 Kaufvorgänge auf, das bedeutet im Schnitt mehr als einen Kaufvorgang pro Woche. 
- Die Altersverteilung zeigt einen schwerpunkt im Bereich zwischen 36 und 50 Jahren auf (vgl. Abbildung 19). Nur $2,5 \%$ der betrachteten Kunden ist jünger als 26 Jahre. Dies ist zum einen auf ein Mindestalter von 18 Jahren für den Erhalt einer Kundenkarte zurückzuführen. Zum anderen könnte ein Grund in den restriktiven Bonitätsüberprüfungen des Kartenausgebers liegen. 17,9: der Kunden sind zwischen 26 und 35 Jahren und $46,3 \div 2$ wischen 36 und 50 Jahren. Mit $33,3 \%$ ist ein relativ großer Anteil älter als 50 Jahre, wovon 8,4 \% sogar älter als 65 Jahre ist. Die fehlende Äquidistanz der Altersklassen für die betrachteten Kunden ergibt sich aus erhebungstechnischen Gründen im Rahmen des Antrages auf eine Kundenkarte. Dasselbe Problem besteht bei den Einkommensklassen.

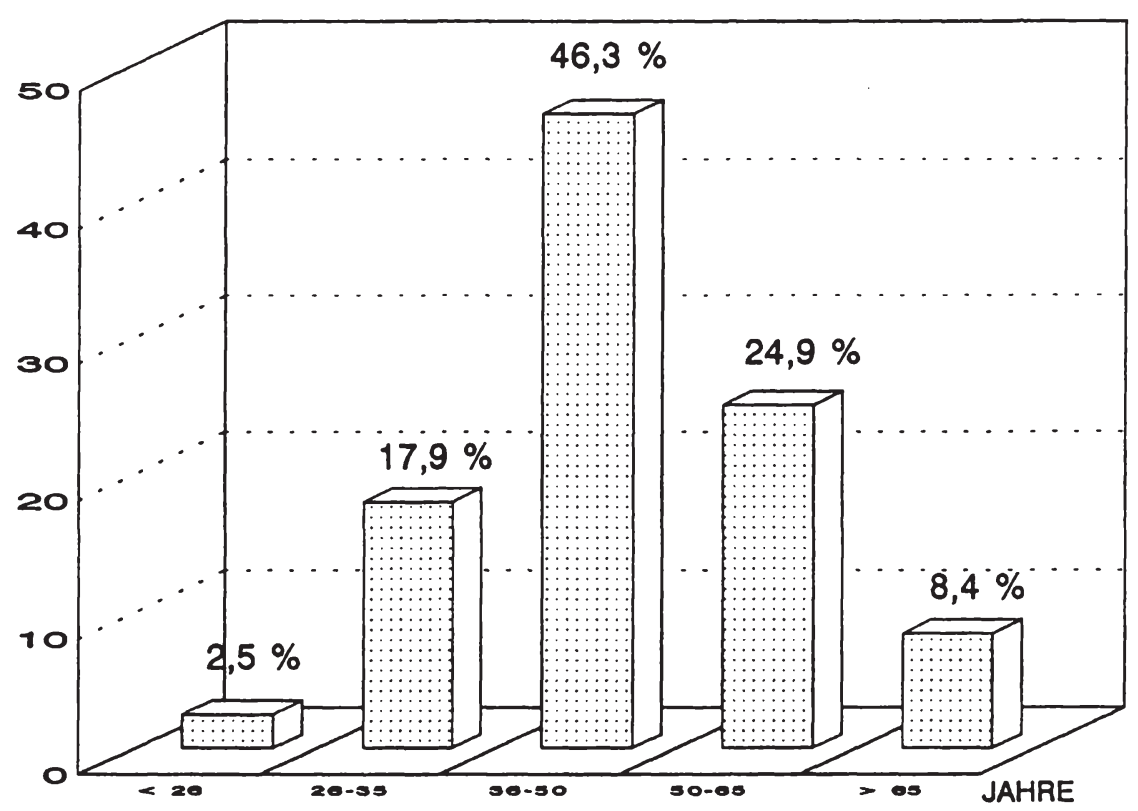

Abb. 19: Altersverteilung in der Gesamtstichprobe 
- Die Einkommensverteilung der betrachteten Kunden zeigt eine Tendenz $z u$ den höheren Einkommensgruppen (vgl. Abbildung 20). Auch dies dürfte in erster Linie auf Bonitätsanforderungen des Kartenausgebers zurückzuführen sein. Darüber hinaus könnte ein Grund darin bestehen, daß höhere Einkommensgruppen tendenziell eine stärkere vertrautheit mit "Plastikgeld" in Form von Kreditkarten haben.

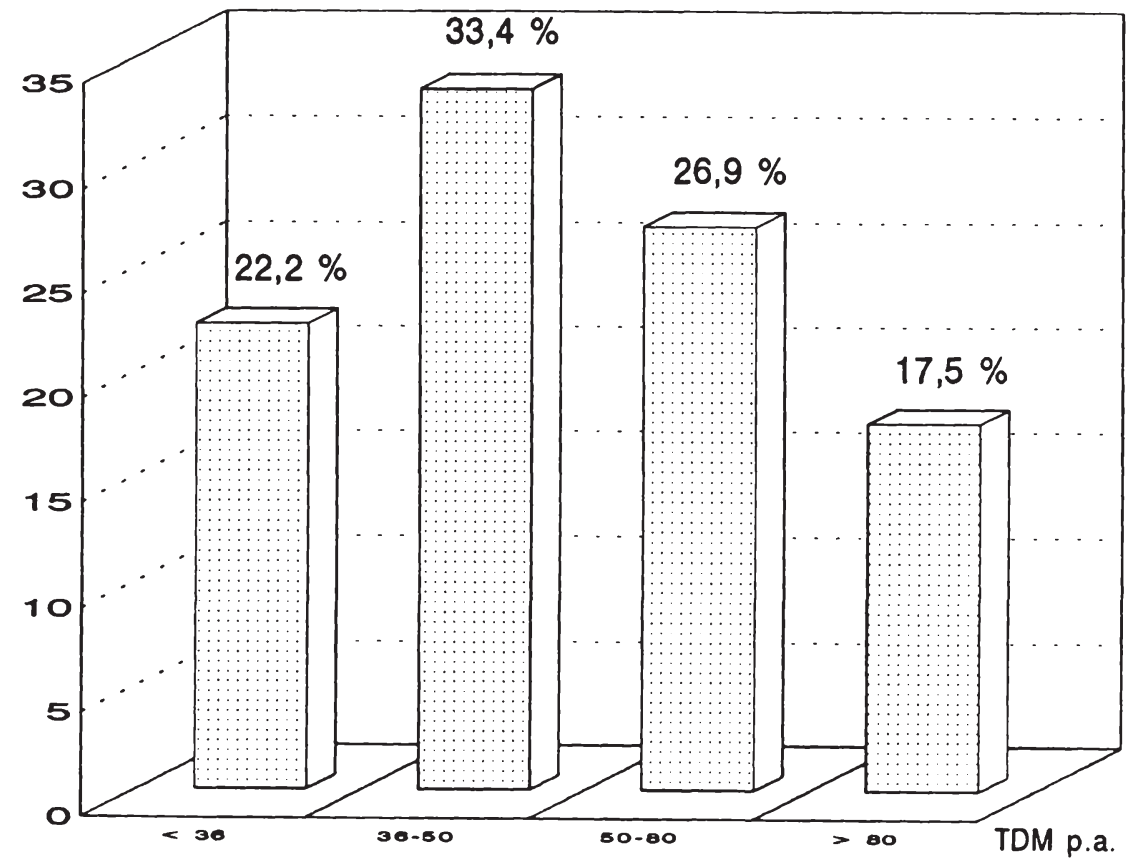

Abb. 20: Einkommensverteilung in der Gesamtstichprobe

- 22,2 $\%$ der Kartenbesitzer haben ein Haushalcsnettoeinkommen unter 36 TDM pro Jahr. 33,4 $\%$ verdienen $\mathrm{zwischen} 36$ und 50 TDM pro Jahr, so daß diese Gruppe ein Drittel der Gesamtstichprobe umfaßt. $26,9 \%$ verdienen $z w i s c h e n 50$ und 80 TDM pro Jahr und mit $17,5 \%$ eine relativ große Gruppe sogar über 80 TDM pro Jahr. 
Insgesamt ergibt sich ein umfangreicher Datensatz für eine detaillierte Erfassung von Verbundbeziehungen. Durch die enthaltenen, wenn auch einfachen demographischen Angaben, ergeben sich auch aufgrund der vorgegebenen Klassen im Alters- und Einkommensbereich bereits Hinweise für eine gruppenbezogene empirische Untersuchung.

\subsection{Rontextdimensionen der analysierten Geschäftsstätte}

Im Rahmen der Kontextdimensionen sind zwei Aspekte abzuhandeln. Zum einen soll die Geschäftsstätte selbst näher definiert und die vorhandene räumliche plazierung von sortimentsbereichen näher beschrieben werden. Dazu treten einige grundsätzliche Angaben über den standort der Geschäftsstätte. Der zweite Aspekt umfaßt eine grobe Analyse der Wettbewerbsbedingungen unterschiedlicher sortimentsbereiche an diesem standort. Aus den beiden Gesichtspunkten können eventuell Hinweise für die Interpretation empirischer Ergebnisse abgeleitet werden. So könnte z.B. das Fehlen eines bestimmten sortimentsbereiches in einer verbundgruppe, das im widerspruch zu einer Hypothese steht, z.B. auf einen intensiven wettbewerb gerade in diesem sortimentsbereich an dem standort zurückgeführt werden. Dies gilt insbesondere, wenn ein dominanter Wettbewerber existieret.

\subsection{Geschäftsstättenbezogene Aspekte}

Der Standort der Geschäftsstätte ist die Fußgängerzone einer Großstadt mit ca. 300.000 Einwohnern. Laut statistischem Jahrbuch $^{1}$ beträgt der Einzelhandelsumsatz ca. 3,7 Mrd. DM. Die Kaufkraft pro Kopf liegt höher als im Bundesdurch-

1 O.V., Statistisches Jahrbuch der Bundesrepublik Deutschland, 0.0., 1991. 
schnitt, so daß grundsätzlich ein gutes Konsumklima vorliegt. Das betrachtete Handelsunternehmen besitzt einen Marktanteil von ca. 4,5\% an diesem Einzelhandelsumsatz. Der Anteil des Umsatzes der Kundenkartenbesitzer am Gesamtumsatz der Filiale beträgt im untersuchten Zeitraum ca. 4,0 \%. In Anbetracht der Tatsache, daß das Kundenkartensystem zu Beginn des Untersuchungszeitraumes noch nicht ganz 2 Jahre besteht, stellt sich diese zahl als relativ hoch dar.

Die Geschäftsstätte selbst hat eine Verkaufsfläche von ca. $16.000 \mathrm{qm}$, die sich auf 6 stockwerke verteilt, wobei jede Etage etwa die gleiche Verkaufsfläche aufweist. ${ }^{2}$ Bei der Auflistung der Zusammenfassung von Abteilungen innerhalb der Stockwerke wird eine Aggregation der Einzelabteilungen zu übergeordneten sortimentsbereichen vorgenommen. Zum einen erhöht dies die übersichtlichkeit der Darstellung. Zum anderen wird damit eine Vergleichsmöglichkeit mit Wettbewerbern geschaffen, da die Untersuchung über den Wettbewerb nur auf diesem Aggregationsniveau durchführbar ist. Eine direkte Identifizierung bestimmter Abteilungen ist beim Wettbewerb kaum möglich. Eine Auflistung der Zusammenstellung von Abteilungen $\mathrm{zu}$ Sortimentsbereichen ist Tabelle $1 \mathrm{zu}$ entnehmen.

- Im Untergeschoß befinden sich die Sortimentsbereiche Haushaltswaren, Glas/Prozellan und Geschenke, Alles für's Bad und Lebensmittel;

- im Erdgeschoß sind die Parfümerie, Uhren und Schmuck, Lederwaren, Schreibwaren, Strümpfe und Handschuhe, Mode-Accessoires sowie der Bereich Foto zu finden;

- im ersten Obergeschoß sind die Sortimentsbereiche Damen oberbekleidung (DOB)-Diverse, DOB-Chosen, DOB-Leder und übrige DOB mit dem Bereich Schuhe zusammengefaßt;

2 Die Daten bzgl. der Geschäftsstätte entspringen den Angaben des untersuchten Handelsunternehmens. 


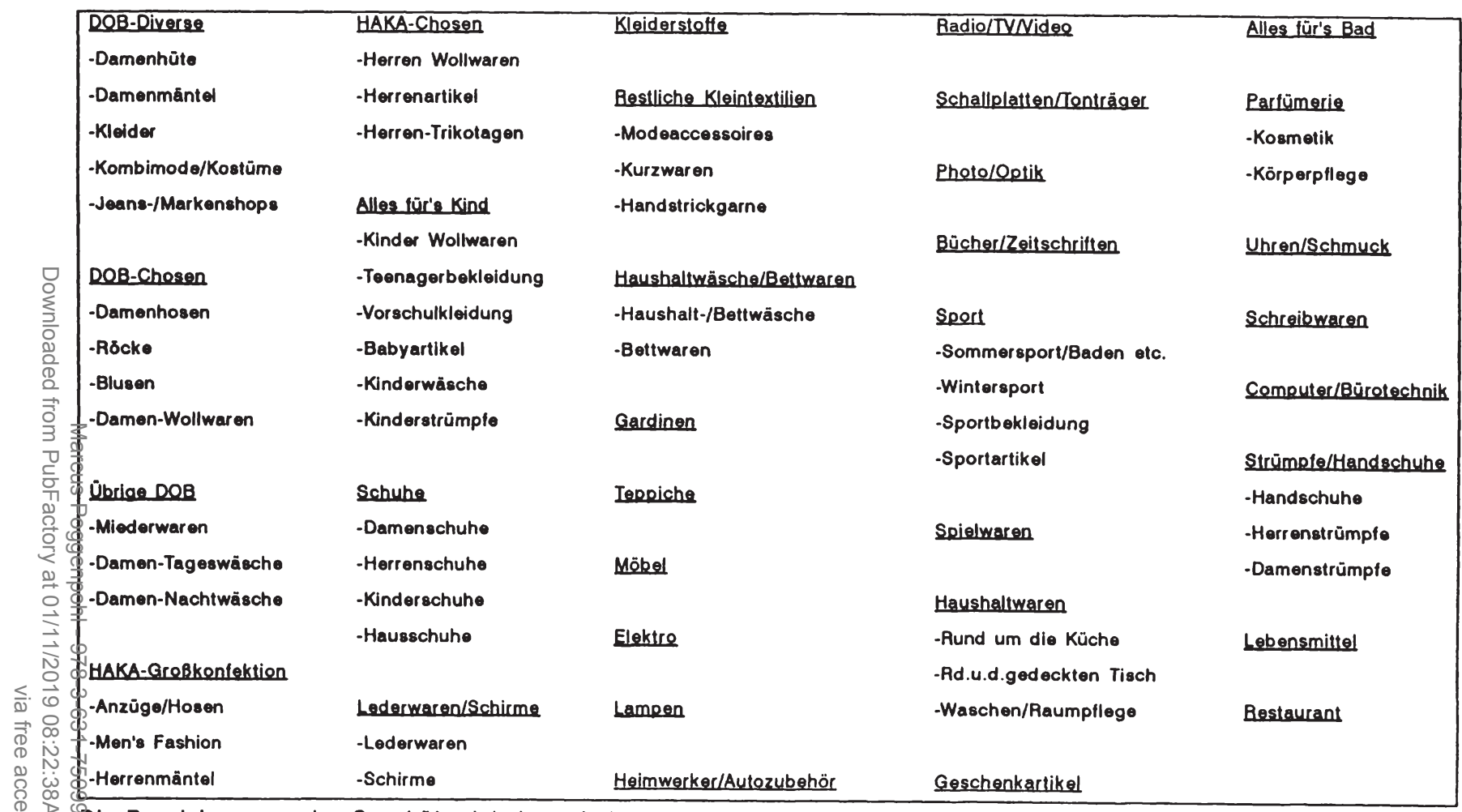

Die Bezeichnungen der Geschäftseinheiten sind unterstrichen

Geschäftseinheiten denen nur eine Abteilung zugeordnet ist, erscheinen ohne Abteilungsliste

Tab. 1: Zuordnung von Abteilungen zu Geschäftseinheiten 
- das zweite Obergeschoß setzt sich aus den Bereichen Herren (HAKA)-Großkonfektion, HAKA-Chosen, Kindersortiment auf der einen Seite und den Bereichen Stoffe, Kurzwaren und Handstrickgarne sowie Haushalt und Bettwäsche auf der anderen seite zusammen;

- im dritten Obergeschoß befinden sich der Bereich Möbel zusammen mit Lampen und Elektroartikel, Gardinen, Bodenbelägen und Teppichen;

- der vierte stock umfaßt die sortimentsbereiche Radio/Fernsehen/Video, Heim- und Handwerk, Schallplatten, Bürotechnik, Bücher, spielwaren sowie den sportbereich.

Bei näherer Betrachtung dieser Zusammenfassungen ist festzustellen, daß das Handelsunternehmen bemüht ist, vom Sortiment her komplementäre Gruppen zusammen zu präsentieren. ${ }^{3}$ Besonders deutlich wird dies im ersten und dritten obergeschoß. Das erste Obergeschoß kann eindeutig mit der Beschriftung "Damenbekleidung" versehen werden. Das dritte Obergeschoß umfaßt ebenso klar ersichtlich den Oberbegriff "Einrichtung". Auch in den anderen stockwerken sind wenn auch nicht eine eindeutige Themensetzung, so doch klar erkennbare Gruppen zu unterscheiden.

Diese themenbezogenen zusammenstellungen werden vermutlich ebenso wie die vom Handelsunternehmen vorgenommene zusammenstellung von Abteilungen zu Sortimentsbereichen das Entstehen bestimmter Verbundbeziehungen fördern. Ebenso ist die physische Trennung des Gesamtsortiments durch das Bestehen verschiedener Etagen vermutlich ein Einflußfaktor auf die Entstehung von Verbundbeziehungen oder für das Fehlen hypothetisch vermuteter Beziehungen. In jedem Fall werden die situativen Voraussetzungen der Geschäftsstätte bei der In-

Eine detaillierte Auflistung der Zuordnung von Abteilungen zu stockwerken ist Tabelle 3 aus Kapitel C.2.2 der vorliegenden Untersuchung $\mathrm{zu}$ entnehmen. 
terpretation der empirischen Ergebnisse in die Betrachtungen einbezogen.

\subsection{Wettbewerbsbezogene Aspekte}

Insgesamt stellt sich die untersuchte Geschäftsstätte, gemessen an der vorhandenen Verkaufsfläche, im Vergleich zu ihren Wettbewerbern sehr positiv dar. ${ }^{4}$ so ist das Handelsunternehmen in 10 Sortimentsbereichen flächengrößter Anbieter und in weiteren 10 Sortimentsbereichen zweitgrößter Anbieter vor Ort. Dies zeigt, daß die Geschäftsstätte zu den dominierenden Handelsgeschäften im Wettbewerb zählt. Die Fläche wird als Indikator herangezogen, weil andere Kennzahlen über Wettbewerber nicht zu beschaffen sind. Darüber hinaus begrenzt die Größe der Verkaufsfläche sowohl den Umfang des Produktangebots als auch die Möglichkeiten der Präsentation. Insgesamt betrachtet hat der flächengrößte Anbieter die besten Chancen im Wettbewerb.

Für eine detailliertere Darstellung der Wettbewerbssituation bietet sich wiederum das Vorgehen nach den bereits vorgestellten Sortimentsbereichen an:

- Im gesamten Bereich der Bekleidung, zu dem die Sortimentsbereiche Damen-, Herren- und Kinderkleidung gehören, gelingt es der Geschäftsstätte in keinem Fall, sich als flächengrößter Anbieter $z u$ profilieren. Dennoch schafft es das Unternehmen bis auf den Sortimentsbereich DOB-Leder, sich unter den drei flächengrößten Anbietern zu positionieren. Es herrscht in diesem Bereich ein intensiver Wettbewerb, vor allem von großen filialisierten Textilhäusern, die quasi als spezialisten der Bekleidungsbran-

4 Die Angaben über den lokalen Wettbewerb entstammen einer Wettbewerbsanalyse des untersuchten Handelsunternehmens. Marcus Poggenpohl - 978-3-631-75099-5 
che weitergehende Möglichkeiten haben als ein Vollsortimenter.

- Im Sortimentsbereich Schuhe, der nicht nach Damen-, Herren- und Kinderschuhen getrennt untersucht wird, ist ebenfalls ein starker Wettbewerb durch spezialisierte Anbieterketten der Schuhbranche zu verzeichnen, so daß hier noch stärkere Defizite $z u$ den flächengrößten Anbietern auftreten als im textilen Bekleidungsbereich.

- Sehr starke Positionen sind dagegen in den Sortimentsbereichen Strümpfe und Handschuhe, Kleiderstoffe, Kleintextilien, Haushalts- und Bettwäsche sowie Gardinen zu verzeichnen. In diesen Bereichen stellt sich die Geschäftsstätte als flächengrößter Anbieter dar. Der Wettbewerb weist aber auch in diesen Bereichen eine andere struktur auf. Die stärksten Mitbewerber sind andere Vollsortimenter, $z u$ denen jeweils kleinere spezialisierte Fachgeschäfte kommen.

- Der Einrichtungsbereich mit den Sortimentsbereichen Teppiche, Bodenbeläge, Möbel, Elektro und Lampen weist erwartungsgemäß bis auf den Bereich Elektro und bedingt auch Lampen keine herausragende Wettbewerbsposition auf. In diesem Bereich sind extrem großflächige Einrichtungs-, Möbel- und Teppichhäuser führend.

- Ebenfalls durch großflächige spezialisten beherrscht stellt sich der Markt für die Sortimentsbereiche Heimwerker/Autozubehör, Radio/Fernsehen/Video, Bürotechnik, Foto und Bücher dar.

- Die Bereiche sport und Spielwaren hingegen haben eine gute Wettbewerbsposition, wobei der sportbereich sogar als flächengrößter Anbieter auftritt. Dies gelingt, obwohl in beiden Bereichen sowohl andere Vollsortimenter 
als auch Spezialisten ein wettbewerbsintensives Umfeld darstellen. Dies gilt auch für die sortimentsbereiche Glas, Porzellan und Geschenke, "Alles für das Bad" und Haushaltswaren.

- Gegenüber Spezialisten und Mitbewerbern, die als Vollsortimenter auftreten, positioniert sich das Handelsunternehmen als flächengrößter Anbieter in den Sortimentsbereichen Uhren und Schmuck sowie Lederwaren. Vor allem im Bereich Uhren und Schmuck erscheint dies vor dem Hintergrund starker Fachhandelsketten erstaunlich.

- Im Bereich Lebensmittel ist die Geschäftsstätte sehr großflächigen Fach- und Supermärkten nicht ebenbürtig. Doch ist hier hervorzuheben, daß sich die Geschäftsstätte schwerpunktmäßig als Nischenanbieter in diesem Bereich profiliert. Dabei wird eher hochwertige und teure ware aus dem Gourmetbereich aufwendig präsentiert.

Insgesamt hat das Handelsunternehmen eine starke Marktposition und kann sich weitgehend im Bereich der jeweils flächengrößten Anbieter etablieren. Nur in den Bereichen, in denen großflächige Spezialisten den Wettbewerb dominieren, kann das Unternehmen keine entsprechende Verkaufsfläche ausweisen.

\subsection{Operationalisierung des verbundbegriffes für die empiri- sche Analyse}

Anhand der in Kapitel B.2.2 dargestellten operationalisierungskriterien wird der Verbund im Rahmen der empirischen Analye wie folgt erfaßt:

- Grundsätzlich geht die Analyse von rein nachfrageinduzierten Verbundbeziehungen aus. Es wirken bei jedem Kauf 
jedoch nicht nur nachfragebezogene Einflußgrößen ${ }^{5}$. Simultan wird der Kauf von Wettbewerbs- und Angebotsbedingungen mitbestimmt. Daher werden z.T. Aspekte aus diesen Bereichen zur Interpretation und zum besseren Verständnis herangezogen, z.B. wird die räumliche Verteilung der sortimentsbereiche innerhalb der Geschäftsstätte betrachtet.

- Einzelne absatzfördernde Maßnahmen, wie z.B. Sonderangebotsaktionen, werden nicht in die Betrachtung einbezogen, da Käufe aus unterschiedlichen Zeitabschnitten gleich behandelt werden. Anders ist eine Erfassung aller Käufe eines ganzen Jahres nicht möglich. Darüber hinaus würde sich vor dem Hintergrund der umfassenden Betrachtung eines Vollsortimenters ein zu komplexes Geflecht einzelner Maßnahmen ergeben. Insgesamt wird daher das absatzpolitische Instrumentarium als konstant angesehen. Gleichzeitig bleiben auch langfristige Faktoren wie bauliche Voraussetzungen und die Verteilung der Sortimentsbereiche innerhalb der Geschäftsstätte konstant. ${ }^{6}$

- Die bestehenden Verbundwirkungen werden als symmetrisch angesehen. Für das Entstehen von Verbundbeziehungen ist es kaum relevant, welcher Kauf einen anderen auslöst.

- Als Aggregationsniveau der Bezugsdaten für die Bestimmung der Verbundbeziehungen wird die Abteilung als Einheit herangezogen. Diese Betrachtungsebene ermöglicht eine Erfassung des Gesamtsortiments und der in ihm bestehenden Beziehungen. Das grundsätzliche Vorgehen und die angewen-

Vgl. Kapitel B.2.1 dieser Untersuchung.

6 Aus der Grundvoraussetzung für die Aufnahme eines Kunden in die betrachtete stichprobe, die darin besteht, daß mindestens zwei Käufe eines Kunden vorliegen müssen, ist bereits abzuleiten, daß der Fall des Kaufs nur eines Gutes nicht in die Betrachtung der Verbundbeziehungen einbezogen wird. Zur Problematik der Ausgrenzung absatzpolitischer Maßnahmen vgl. Kapitel B.2.21. 
deten Verfahren werden jedoch von der Wahl des Aggregationsniveaus nicht berührt. Anhand des empirischen Vorgehens können zum Beispiel auch Teilsortimente auf Artikeloder Artikelgruppenniveau bzgl. des Bestehens von Verbundbeziehungen analysiert werden. Das für die Wettbewerbsanalyse verwendete sortimentsbereichsniveau dagegen wird nur als vergleichendes Element in die Interpretation einbezogen.

- Die Verbundanalyse wird in der empirischen Untersuchung anhand von Absatzgrößen durchgeführt. Im Rahmen der Erfassung des Kaufverbundes wird der Kauf in Abteilung $A$ und Abteilung $B$ somit als 1 gesetzt. Dies geschieht unabhängig von den betrachteten Abteilungen und den Kaufsummen, die durch die einzelnen Käufe repräsentiert werden.

Gleiches gilt auch für den Nachfrageverbund, bei dem jeder Kauf als eine Einheit unabhängig von der Kaufsumme einbezogen wird. Auch die berechneten Intensitätswerte sind ausschließlich von Absatzzahlen beeinflußt.

Für die Analyse des Kauf- und Nachfrageverbundes werden unterschiedliche multivariate Analyseverfahren eingesetzt. 7 Die Cluster-, Diskriminanz- und Faktorenanalysen wurden auf einem 486er PC mit Hilfe des Programms SPSS unter dem Betriebssystem os 2 durchgeführt.

\subsection{Festlegung der Untersuchungsgruppen nach demographischen und ökonomischen Kriterien}

Grundsätzlich eignen sich die Kriterien Alter und Einkommen als Ausgangspunkt für die Festlegung von Untersuchungsgruppen. Auf den ersten Blick bietet sich die Erstellung einer 
Matrix aus diesen beiden Kriterien an. Da es fünf Altersklassen und vier Einkommensklassen gibt, würde sich daraus eine 20 Felder Matrix herleiten. Daraus resultierend müßten 20 einzelne Gruppen untersucht werden.

Die zusammensetzung der stichprobe läßt dieses Vorgehen jedoch als ungeeignet erscheinen. Wie aus Abbildung 21 ersichtlich ist, sind die Kaufakte innerhalb der unterschiedlichen Altersklassen sehr ungleich verteilt. Dies resultiert zum Teil aus den nicht äquidistanten Gruppendefinitionen, die durch den Datensatz vorgegeben werden.

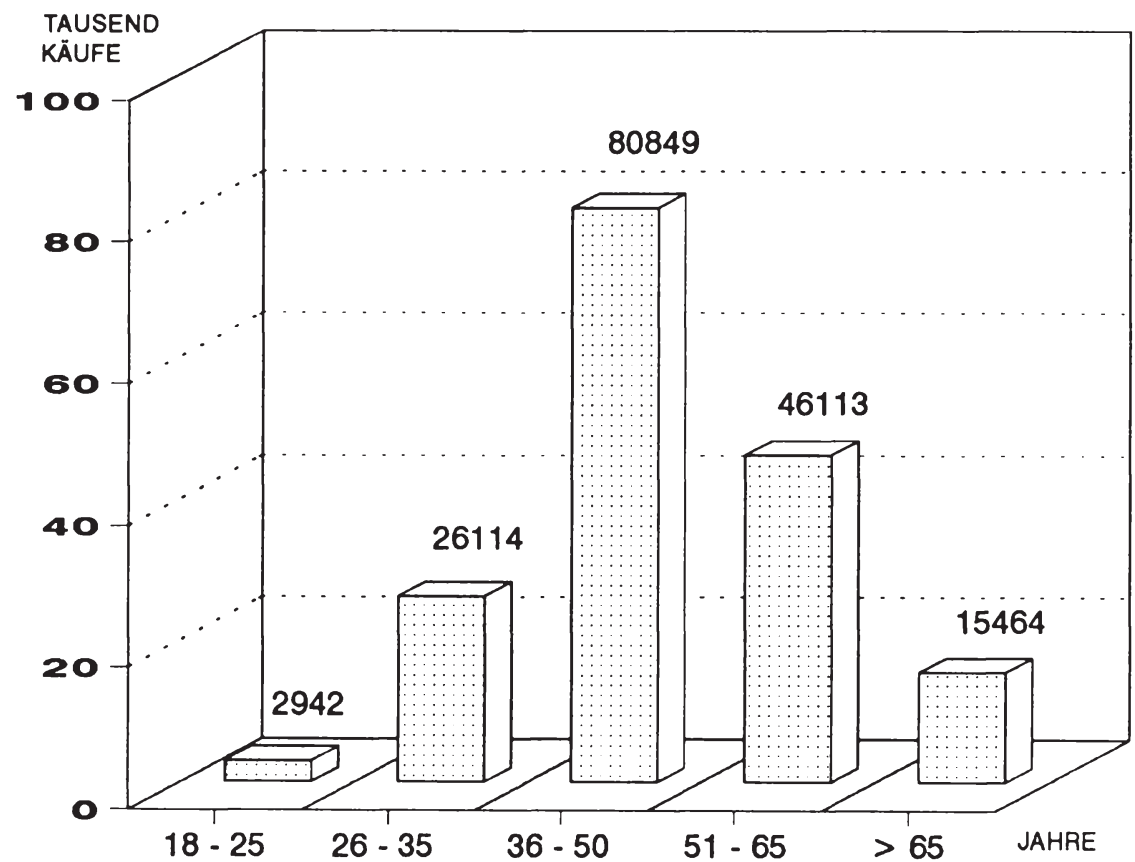

Abb. 21: Verteilung von Käufen in den Altersklassen

So ist die Gruppe der bis zu 25 Jahre alten Kundenkartenbesitzer nur mit 2.942 Kaufakten vertreten. Diese sehr kleine stichprobe würde sich durch eine nochmalige Einteilung in 
Einkommensklassen noch stärker verringern. Auch wenn die Einkommensverteilung in diesem Bereich nicht zu weit streuen sollte, so ist eine Verringerung der stichprobe in jeder Form abzulehnen.

Eine Zusammenlegung dieser Altersgruppe mit den 26-35 Jahre alten Kundenkartenbesitzern würde hingegen eine Möglichkeit darstellen, die stichprobe zu vergrößern.

Aus diesem Grund werden die Untersuchungsgruppen nicht nach der 20-Felder-Matrix festgelegt. Stattdessen werden Gruppen getrennt zum einen nach Altersklassen und zum anderen nach Einkommensklassen untersucht. Dabei lassen sich Unterschiede in den Verbundbeziehungen sowohl bei Alters- als auch bei Einkommensgruppen feststellen.

Für die empirischen Untersuchungen im Rahmen dieser Arbeit ergeben sich daher trotz des großen Datenumfanges aufgrund der komplexen Zusammenhänge bei der Verbundmessung und den daraus resultierenden komplexen Verfahren Restriktionen. Obwohl z.B. für die Kaufverbundanalyse mehr als 50.000 Raufpaare abgeleitet und in die Matrix eingebracht werden können, ist eine Unterteilung in Untergruppen nicht möglich, da dann die zellen der Matrix nur noch mit statistisch unzureichenden Fallzahlen gefüllt werden können.

Dies ergibt sich daraus, daß eine 67 x 67 Matrix aufgebaut werden muß. Auch wenn diese aufgrund der symmetrievoraussetzung nur zur Hälfte gefüllt wird, ergibt sich für die Grundgesamtheit eine durchschnittliche Zellenbesetzung von ca. 24 Kaufpaaren bei $2.145 \mathrm{zu}$ besetzenden Zellen. Daher wird die Kaufverbundanalyse nur auf der Basis der Grundgesamtheit durchgeführt. Dies genügt jedoch, um das Verfahren grundsätzlich zu testen und durchzuführen, wobei gruppenspezifische Analysen späteren Forschungen auf der Basis noch umfangreicherer Datenbestände vorbehalten bleiben. 
Die Nachfrageverbundanalyse dagegen kann auch in Untergruppen stattfinden. Die einzige Einschränkung für diese Analyse besteht darin, daß die unterste Altersgruppe (18-25 Jahre) mit $2,5 \%$ und die oberste Altersgruppe (über 65 Jahre) mit $8,4 \div z u$ gering besetzt sind. Daher werden sie jeweils der benachbarten Gruppe zugerechnet. Daraus ergibt sich eine Einteilung in drei Altersklassen. Die erste umfaßt das Alter von 18-35 Jahren $(20,4 \%$ der Gesamtstichprobe), die zweite das Alter von 36-50 Jahren $(46,3 \%$ der Grundgesamtheit) und die dritte Gruppe das Alter über 50 Jahre $(33,3 \&$ der Gesamtstichprobe). Dadurch ergeben sich ausreichende Fallzahlen pro Untersuchungsgruppe, die eine gesicherte empirische Analyse zulassen.

\section{Der Raufverbund als zeitpunktbezogener Ansatz der Ver- bundmessung}

Zur Ermittlung des Kaufverbundes werden alle Käufe analysiert, bei denen der Kartenkunde in mehr als einer Abteilung eingekauft hat. Die in Kaufpaare zerlegten Kaufakte werden in einer Matrix aggregiert ${ }^{8}$ und in eine clusteranalyse überführt, deren Ergebnis anhand einer graphischen Analyse des Dendrogramms vorgenommen wird.

Die graphische Analyse zeigt über das Bestehen von Kaufverbundbeziehungen hinaus auch deren Intensität, da das Aggregationsniveau jeweils ablesbar ist. Im folgenden werden drei extreme Arten des Aggregationsniveaus unterschieden. Diese Extrema kennzeichnen die Besonderheiten der Kaufverbundbeziehungen am deutlichsten. Dies sind Kerngruppen, Randgruppen und Einzelabteilungen. Andere zwischen den Extrema liegende Niveaus werden je nach Intensität beschrieben. 
- Kerngruppen sind die auf niedrigstem Niveau fusionierende Abteilungen. Abteilungen, die zu diesen Gruppen gehören, weisen die stärksten Kaufverbundbeziehungen auf. Diese Gruppen sollten wenn möglich auch bei Marketingentscheidungen als stabil angesehen, d.h. zum Beispiel nicht räumlich getrennt werden. Auch bei Eliminierungsentscheidungen sollte die enge Beziehung dieser Abteilungen berücksichtigt werden.

- Randgruppen bestehen aus Abteilungen, die zwar untereinander relativ eng durch einen Kaufverbund miteinander in Beziehung stehen. Jedoch sind sie relativ schwach an die restlichen Abteilungen ihres clusters angebunden. Dies können maximal zwei bis drei Abteilungen sein. Diese Kombination ist also in sich stabil und sollte auch so behandelt werden, doch ihre schwache Anbindung an ihr Kaufverbundcluster stellt ein gewisses Flexibilitätspotential dar.

- Einzelabteilungen weisen zu keiner zweiten Abteilung starke oder auch nur relativ starke Kaufverbundbeziehungen auf, so daß sie am ehesten von den anderen Abteilungen eines clusters getrennt werden könnten, ohne zu starke Auswirkungen auf andere Abteilung erwarten zu lassen.

\section{1 sortimentsgruppenbildung und Verbundintensität in der Gesamtstichprobe}

Insgesamt ergeben sich bei der Durchführung der Clusteranalyse neun cluster (siehe Abbildung 22). Die Abteilungskombinationen und -gruppierungen erscheinen dabei überwiegend sachlogisch und sind folgendermaßen zu interpretieren:

Marcus Poggenpohl - 978-3-631-75099-5 


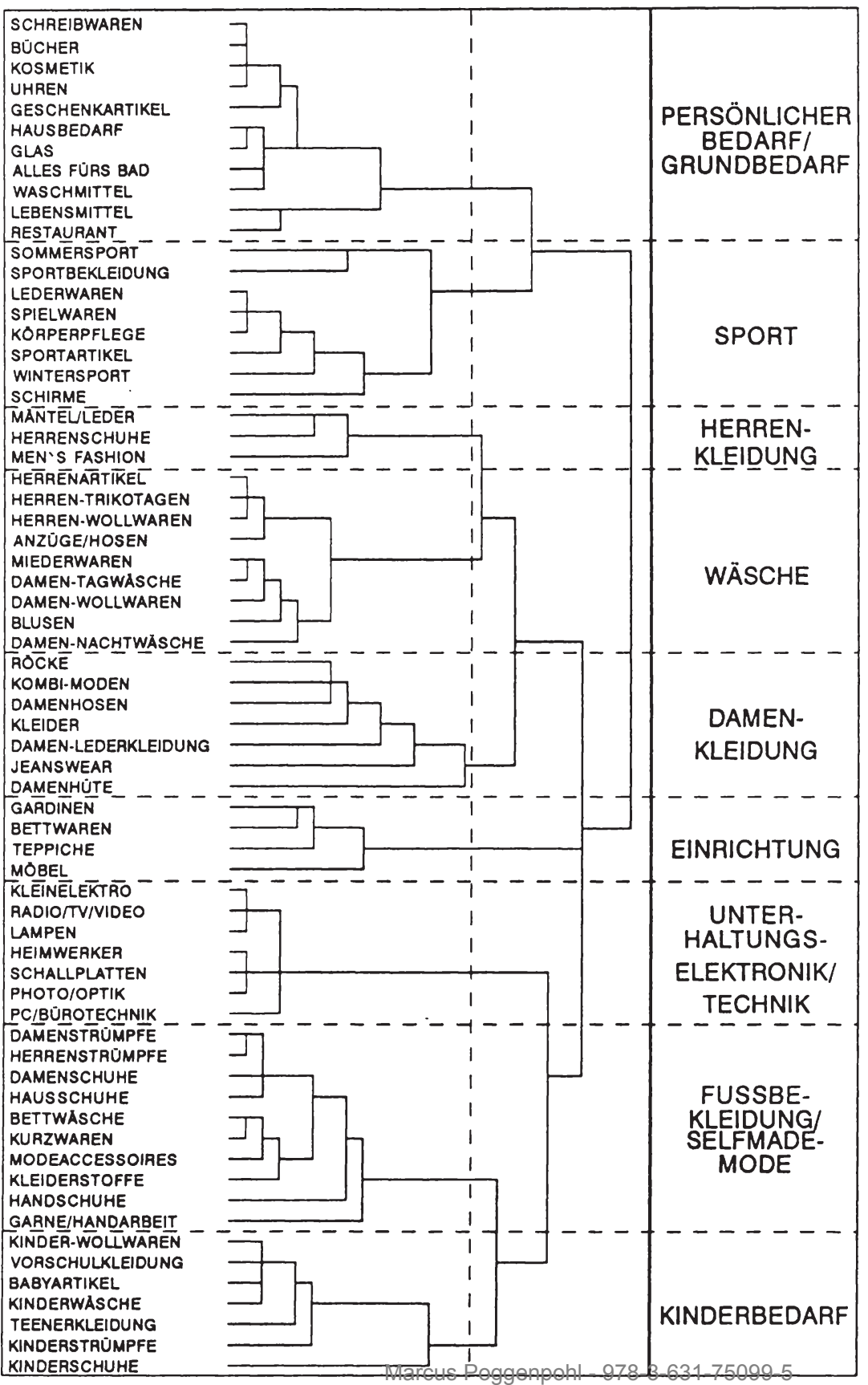




\section{Cluster 1: Grundbedarf und persönlicher Bedarf}

Dieses Cluster weist zunächst einmal zwei Kerngruppen auf. Die erste Kerngruppe besteht aus den vier Abteilungen Schreibwaren, Bücher, Kosmetik und Uhren/Schmuck. Diese Kerngruppe weist noch eine mittelstarke Bindung $z u$ der Abteilung Geschenkartikel auf.

Die zweite Kerngruppe umfaßt die zwei Abteilungen "Rund um die Küche", die den Haushaltsbedarf beinhaltet und "Rund um den gedeckten Tisch" mit den Schwerpunkten auf Glas, Geschirr etc. Die beiden letztgenannten sind noch relativ eng mit den Abteilungen Wasch- und Reinigungsbedarf sowie "Alles für's Bad" verbunden, so daß dort die Grundausstattung eines Haushalts zusammenfließt. Darüber hinaus weist dieses mit 11 Abteilungen größte Cluster noch eine Randgruppe mit den Abteilungen Lebensmittel und Restaurant auf.

\section{cluster 2: sport}

Dieses Cluster umfaßt insgesamt 8 Abteilungen, wobei alle Sportabteilungen enthalten sind. Allerdings enthält das cluster eine Kerngruppe, die nicht primär auf den sport augerichtet erscheint, aber doch dem Freizeitbereich (Spielwaren) oder Pflegebereich (Körperpflege), der mit dem Sport verbunden ist, enthält. Die Kerngruppe besteht aus den Abteilungen Lederwaren, Spielwaren und Körperpflege. Am engsten mit dieser Kerngruppe ist die Abteilung sportartikel verbunden, so daß eine gewisse Hartwarenausrichtung bis zu dieser stufe des clusters $z$ u verzeichnen ist. Die eher bekleidungsorientierten sportabteilungen mit sommersport inkl. Strand- und Bademoden, Wintersport und Sportbekleidung stehen eher isoliert und fließen z.T. erst auf dem Niveau von Einzelabteilungen zusammen. Dies verwundert vor der Saisonabhängigkeit des Winter- und Sommersports, deren Sortimente z.T. im Wechsel auf derselben Verkaufsfläche angeboten werden und der gleichzeitigen Zeitpunktorientierung des Kaufverbundes nicht. Dies erklärt wohl auch die inhaltlich eher 
lose angebundene Kerngruppe dieses Clusters. Als Einzelabteilung sind darüber hinaus noch die Schirme an dieses cluster angebunden.

\section{Cluster 3: Herrenkleidung}

Dieses cluster umfaßt insgesamt nur drei Abteilungen, die auf eher mäßigem Niveau durch Kaufverbundbeziehungen zusammengefaßt werden. Diese Abteilungen sind Herrenmäntel, Herrenschuhe und Men's Fashion. Untersucht man zum einen die weitere Anbindung dieses clusters an andere und zum anderen deren inhaltliche zusammensetzung so ist festzustellen, daß das cluster auf einem etwas schwächeren Niveau als die Grenze für eine clustertrennung mit dem cluster Wäsche verbunden ist. In diesem cluster befinden sich alle übrigen "Herrenabteilungen", insbesondere wäsche und Herrenartikel, so daß die Kaufverbindungen $\mathrm{zu}$ diesen Abteilungen wenn auch schwach, so doch vorhanden sind.

Zudem enthält dieses cluster exakt die Abteilungen, die zwecks einer Anprobe die Anwesenheit des späteren Trägers der Bekleidung voraussetzen. Dies ist bei der Herrenwäsche, den -strümpfen und -artikeln nicht unbedingt notwendig. Daher werden diese Sortimentsbereiche häufig von anderen Personen (Ehegatten, Mütter etc.) zusammen mit ihrem Bedarf erworben, so daß die gesamte Konstellation der Abteilungskombinationen in bezug auf den Herrenbereich durchaus hoch plausibel ist.

\section{Cluster 4: Wäsche}

Insgesamt umfaßt dieses cluster neun Abteilungen. Trotzdem die Verbindungen innerhalb dieses clusters insgesamt sehr eng sind, lassen sich dennoch ganz klar zwei Bereiche voneinander trennen, die mit Herren- bzw. Damenbedarf überschrieben werden können. Im Bereich des Herrenbedarfs befindet sich eine Kerngruppe aus den drei Abteilungen Herrenartikel, Herrentrikotagen und Herren-Wollwaren, an die etwas 
schwächer die Abteilung Anzüge/Hosen des Herrenbereichs angebunden ist. 9

Im Bereich des Damenbedarfs ist ebenfalls eine Kerngruppe festzustellen, die allerdings nur aus zwei Abteilungen besteht. Dies sind die Miederwaren und die Damen-Tageswäsche. $\mathrm{Zu}$ dieser Kerngruppe bestehen Kaufverbindungen auf jeweils minimal schwächerem Niveau $z u$ den drei Abteilungen DamenWollwaren, Blusen und Damen-Nachtwäsche. Auf mittlerem Niveau fließen dann die Bereiche des Herren- und Damenbedarfs zusammen.

\section{Cluster 5: Damenkleidung}

In diesem cluster werden 6 Abteilungen zusammengefaßt. Trotzdem alle in dieser Gruppierung enthaltenen Abteilungen eindeutig dem Bereich der Damenoberbekleidung zugerechnet werden können, sind die Niveaus der Kaufverbundenheit doch eher als schwach zu bezeichnen. So ist die stärkste Verbindung dieser Gruppe gerade so intensiv wie die Verbindung des Damen- und Herrenbedarfs in cluster 4. Die schwächsten Verbindungen liegen eindeutig im Bereich von Einzelabteilungen. Dies kann daran liegen, daß unterschiedliche Abteilungen des Damenoberbekleidungsbereiches durchaus sortimente mit Schwerpunkten für bestimmte Altersgruppen aufweisen. So ist es z.B. unwahrscheinlich, daß die Junge Mode aus der Abteilung Jeanswear und Markenshops zusammen (und dann noch zu einem zeitpunkt) mit einem Damenhut erworben wird, da die Sortimente für entgegengesetzte Altersgruppen konzipiert werden.

Für diese Argumentation spricht auch die Gruppe der drei Abteilungen mit den stärksten Kaufverbindungen innerhalb dieser Gruppe. Dies sind die Abteilungen Röcke, Jacken/ Kostüme/Kombinationsmode und Damenhosen. Von diesen drei Da- 
menoberbekleidungsabteilungen ist eine Ausrichtung auf dieselbe Altersgruppe vorstellbar.

\section{cluster 6: Einrichtung}

Dieses cluster beinhaltet nur vier Abteilungen, die alle auf mittlerem Niveau miteinander verbunden sind. Dies sind die Abteilungen Gardinen und Bettwaren (Matratzen etc.), die auch die engste Verbindung aufweisen sowie Teppiche und Möbel, wobei die Möbel am schwächsten angebunden sind. Hierzu ist allerdings anzumerken, daß die Abteilung Möbel bereits eine Aggregation aus weiter spezialisierten Bereichen wie z.B. Wohnraum-, Küchen-, Schlafraummöbel etc. darstellt. In diesen Abteilungen ergaben sich so geringe Fallzahlen, daß diese zu einem umfassenden Möbelbereich aggregiert wurden. Dabei wurden allerdings eventuelle enge Beziehungen von spezialbereichen (z.B. Wohnraummöbel und Teppiche) zerstört, so daß bei der aggregierten Möbelabteilung nur diese eher schwache Anbindung besteht.

\section{cluster 7: Unterhaltungselektronik/Technik}

Mit den Verbindungen der sieben in diesem cluster enthaltenen Abteilungen ergibt sich insgeamt das cluster mit der höchsten Dichte, d.h. den stärksten Kaufverbundbeziehungen. Zunächst enthält dieses cluster zwei Kerngruppen aus jeweils drei Abteilungen. Die erste Kerngruppe enthält mit den Abteilungen Kleinelektro, Radio/Fernsehen/Video und Lampen sehr stark elektronikorientierte sortimentsbereiche. Die zweite Kerngruppe enthält die Abteilungen Heimwerker/Autozubehör, Schallplatten/Tonträger und Photo/optik, die ebenfalls sehr stark technikorientiert sind und dem Hobbybereich zugeordnet werden können. Beide Kerngruppen sind darüber hinaus auf einem relativ starken Kaufverbundniveau miteinander verbunden. Zusätzlich kommt zu dieser Kombination auf dem gleichen Kaufverbundenheitsniveau noch die Abteilung Computer/Bürotechnik $z u$ diesem cluster. Insgesamt 
ergibt sich also eine hochgradig kaufverbundene Abteilungskombination, die eine hochplausible Gruppierung darstellt.

\section{Cluster 8: Selfmade-Mode/Fußbekleidung}

In diesem mit 10 Abteilungen zweitgrößten cluster sind zwei Untergruppen zu je vier Abteilungen und zwei eher schwach angebundene einzelne Abteilungen festzustellen. Die erste Untergruppe umfaßt mit den Abteilungen Damen- und Herrenstrümpfe, die gleichzeitig eine Kerngruppe bilden sowie Damenschuhe und Hausschuhe vier Sortimente, die alle dem oberthema "Fußbekleidung" zugeordnet werden können.

Die zweite Gruppe scheint eher auf das "Selbstanfertigen" von Kleidern ausgerichtet $\mathrm{zu}$ sein. Störend in diesem Kontext ist allerdings auf den ersten Blick die Abteilung Haushaltsbzw. Bettwäsche, obwohl sich auch in diesen Abteilungen Bereiche befinden, die zum Oberthema passen. Die Abteilungen Bettwäsche und Kurzwaren bilden dabei eine Kerngruppe, der die Abteilungen Modeaccessoires und Kleiderstoffe zugeordnet werden.

Die beiden einzelnen Abteilungen, die erst auf einem mäßigen Kaufverbundenheitsniveau $\mathrm{zu}$ diesem Cluster stoßen, sind die Handschuhe sowie die Handstrickgarne, die wiederum thematisch zur Selbstanfertigung von kleidung passen.

\section{Cluster 9: Rinderbedarf}

Das letzte cluster umfaßt alle sieben Abteilungen, die dem Bereich Kinderbedarf zugeordnet werden können. Bis auf die Kinderschuhe, die eine Einzelabteilung darstellen, sind die Kaufverbindungen innerhalb dieses clusters relativ dicht. Die zentrale Gruppe dieses Clusters besteht aus vier Abteilungen, die fast so eng wie eine Kerngruppe miteinander verbunden sind. Dazu gehören die Kinder-Wollwaren, die Vorschul- und Kinderbekleidung, die Babyartikel und die Kinderwäsche. 
All diese Abteilungen sind auf ein Kindesalter bis ca. 6 Jahren ausgelegt und somit sind die Sortimente relativ fokussiert. Daraus resultiert wahrscheinlich auch die etwas schwächere Anbindung der Abteilung Teenagerbekleidung, die auf eine andere Altersgruppe ausgerichtet ist. Wenn also eine Familie nicht gerade mehrere kinder hat, ist die Verbindung zwangsläufig schwächer. Die letzte aufzuführende Abteilung sind die Kinderstrümpfe, die sogar noch etwas schwächer an die zentrale Gruppe angebunden sind als die Teenagerbekleidung.

Insgesamt spiegeln die bei dieser Analyse aufgedeckten Kaufverbundbeziehungen weitgehend sehr plausible Kaufverhaltensmuster wider. Die z.T. klaren Abgrenzungen thematisch in sich hoch homogener Gruppen (z.B. Cluster 9 Kinderbedarf oder cluster 7 Unterhaltungselektronik/Technik) sind eindeutig interpretierbar. Aber auch auf den ersten Blick überraschende Trennungen z.B. der Herrenoberbekleidung (Cluster 3) und des übrigen Herrenbedarfs (Cluster 4) sind durch die Betrachtung bestimmter Kaufverhaltensmuster plausibel zu erklären.

Insofern hat sich das gewählte Vorgehen zur Ermittlung von Kaufverbundbeziehungen bei einem Vollsortimenter auf Abteilungsniveau als ebenso trag- wie aussagefähig erwiesen. Dadurch erschließen sich für die Entscheidungsfindung im Handelsmarketing große Informationspotentiale, die entscheidungsunterstützenden charakter haben.

Darüber hinaus können relativ einfach detailliertere Aussagen gewonnen werden, wenn auf der Basis eines größeren Kartenbesitzerstammes und damit auf der Basis eines umfangreicheren Datenpools auch statistisch haltbare Analysen in demographischen Untergruppen durchgeführt werden können. 
2.2 Überprüfung der Einflüsse der Kontextdimensionen der Geschäftsstätte auf die sortimentsgruppenbildung

An dieser stelle wird überprüft, inwiefern der Geschäftsstättenaufbau das zustandekommen der Verbundbeziehungen im Kaufverbund beeinflußt und ob wiederum Wettbewerbsaspekte als Erklärung für Kaufverbundbeziehungen herangezogen werden können.

In diesem Zusammenhang wird die Arbeitshypothese $\mathbf{H}_{\mathbf{R A I I}}$ überprüft:

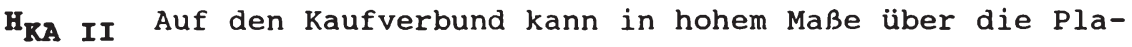
zierung der Abteilungen innerhalb der Geschäftsstätte Einfluß genommen werden.

Um einen Vergleich zwischen der clusterzusammensetzung und der räumlichen Anordnung der Abteilungen durchzuführen, wird überprüft, wie sich die zu einem cluster gehörenden Abteilungen auf die verschiedenen stockwerke der Geschäftsstätte verteilten. 10

- Cluster 1:

Verteilung auf Untergeschoß und Erdgeschoß. Eine Ausnahme bildet das Restaurant, das eine sonderstellung besitzt.

- cluster 2:

Der sportbereich befindet sich im 4. Stock. Alle anderen Abteilungen liegen im Erdgeschoß.

10 Einen Überblick über die Lage der Abteilungen in der Geschäftsstätte gibt Tabelle 2. Die Clusterzugehörigkeit steht jeweils in Klammern hinter der Abteilungsbezeichnung. 


\begin{tabular}{|c|c|c|c|}
\hline & $\begin{array}{l}\text { SOMMERSPORT(2) } \\
\text { SPORTBEKLEIDUNG(2) } \\
\text { SPORTARTIKEL(2) } \\
\text { WINTERSPORT(2) } \\
\text { SPIELWAREN(2) }\end{array}$ & $\begin{array}{l}\text { ELEKTRO(7) } \\
\text { RADIO/TV/VIDEO(7) } \\
\text { SCHALLPLATTEN(7) } \\
\text { COMPUTER(7) } \\
\text { PHOTO/OPTIK(7) } \\
\text { HEIMWERKER(7) }\end{array}$ & RESTAURANT(1) \\
\hline & $\begin{array}{l}\text { BETTWAREN(6) } \\
\text { MOBEL(6) } \\
\text { LAMPEN(7) }\end{array}$ & $\begin{array}{l}\text { GARDINEN(6) } \\
\text { TEPPICHE(6) }\end{array}$ & \\
\hline & $\begin{array}{l}\text { ANZÜGE/HOSEN(4) } \\
\text { MEN'S FASHION(3) } \\
\text { HERRENMÄNTEL(3) } \\
\text { HERRENARTIKEL(4) } \\
\text { HERREN-TRIKOTAGEN(4) } \\
\text { HERREN-WOLLWAREN(4) }\end{array}$ & $\begin{array}{l}\text { KINDER-WOLLWAREN(9) } \\
\text { VORSCHULBEKLEIDUNG(9) } \\
\text { BABYARTIKEL(9) } \\
\text { KINDERWÄSCHE(9) } \\
\text { TEENERBEKLEIDUNG(9) } \\
\text { KINDERSTRÜMPFE(9) }\end{array}$ & \\
\hline & $\begin{array}{l}\text { MIEDERWAREN(4) } \\
\text { DAMEN-TAGESWASCHE(4) } \\
\text { DAMEN-NACHTWÄSCHE(4) } \\
\text { DAMEN-WOLLWAREN(4) } \\
\text { HANDSTRICKGARNE(8) } \\
\text { STOFFE(8) } \\
\text { KURZWAREN(8) }\end{array}$ & $\begin{array}{l}\text { BLUSEN(4) } \\
\text { ROCKE(5) } \\
\text { KOMBI-MODEN(5) } \\
\text { DAMEN-HOSEN(5) } \\
\text { KLEIDER(5) } \\
\text { DAMENMANTEL(5) } \\
\text { JEANSWEAR(5) }\end{array}$ & $\begin{array}{l}\text { DAMENSCHUHE(8) } \\
\text { HERRENSCHUHE(3) } \\
\text { KINDERSCHUHE(9) } \\
\text { HAUSSCHUHE(8) } \\
\text { MODEACCESS.(8) } \\
\text { BETTWÄSCHE(8) }\end{array}$ \\
\hline & $\begin{array}{l}\text { DAMENSTRŨMPFE(8) } \\
\text { HERRENSTRÜMPFE(8) } \\
\text { HANDSCHUHE(8) } \\
\text { LEDERWAREN(2) } \\
\text { SCHIRME(2) }\end{array}$ & $\begin{array}{l}\text { UHREN/SCHMUCK(1) } \\
\text { KOSMETIK(1) } \\
\text { KORPERPFLEGE(2) }\end{array}$ & $\begin{array}{l}\text { BÜCHER(1) } \\
\text { SCHREIBWAREN(1) }\end{array}$ \\
\hline & \multicolumn{2}{|c|}{$\begin{array}{l}\text { GESCHENKARTIKEL(1) } \\
\text { GLAS/PORZELLAN(1) } \\
\text { ALLES FUR'S BAD(1) } \\
\text { HAUSBEDARF(1) } \\
\text { WASCH- UND REINIGUNGSMITTEL(1) }\end{array}$} & LEBENSMITTEL(1) \\
\hline
\end{tabular}

OG=OBERGESCHOSS EG=ERDGESCHOSS UG=UNTERGESCHOSS DIE ZAHLEN IN KLAMMERN GEBEN DIE CLUSTERZUGEHÖRIGKEIT AN

Tab. 2: Zuordnung von Abteilungen zu Stockwerken

- Cluster 3:

Alle Abteilungen liegen im 2. Obergeschoß. Ausnahme sind die Herrenschuhe mit ihrer Angliederung an den zentralen Schuhbereich. 
- Cluster 4:

Die Abteilungen des Herrenbedarfs sind im 2. Stock, die des Damenbedarfs im 1. Stock.

- Cluster 5:

Alle Abteilungen liegen im 1 . Obergeschoß.

- Cluster 6:

Alle Abteilungen befinden sich im 3. Obergeschoß.

- Cluster 7:

Alle Abteilungen sind im 4. Stock. Nur die Abteilung Lampen liegt im 3 . Obergeschoß.

- Cluster 8:

Die Abteilungen verteilen sich auf zwei Gruppen. Eine Gruppe befindet sich im Erdgeschoß, die andere im 1. Obergeschoß.

- Cluster 9:

Alle Abteilungen sind im 2. Obergeschoß. Eine Ausnahme bilden wie in cluster 3 die Schuhe mit ihrer zuordnung zu einer zentralen Schuhabteilung.

Insgesamt zeigt diese vergleichende Analyse, daß hochgradige Überschneidungen $\mathrm{zwischen}$ räumlicher zuordnung und bestehenden Kaufverbundbeziehungen existieren. Allerdings sind ebenso thematische Homogenitäten innerhalb der cluster gegeben. Darüber hinaus versucht der Handel, nicht analytisch, sondern durch Erfahrung gestützt, Abteilungen im sinnvollen Kontext zu plazieren. Der Ursache-Wirkungs-Zusammenhang zwischen Plazierung und Kaufverbund ist auf Basis der vorliegenden Daten nicht endgültig zu klären. Dies liegt vor allem in der simultanen wirkung aller Einflußbereiche (Ange- 
bot/Wettbewerb/Konsument) begründet. 11 Die Isolierung einer einzelnen Einflußgröße ist sehr problematisch. Die Analogien sind jedoch evident. Insofern kann die einzelne Einflußgröße Plazierung bei der situativen Größe Kaufverbund als dominierend für das Zustandekommen von Verbundbeziehungen angesehen werden. Daher gilt die Hypothese $\mathbf{H}_{\text {RAII }}$ als bestätigt. Dieses Ergebnis wäre jedoch in weitergehenden Forschungsvorhaben kritisch zu überprüfen.

Direkte Auswirkungen der Wettbewerbsgrößen sind bis auf die mögliche Auswirkung z.B. des starken Wettbewerbs im Möbelbereich, der eventuell zur schwächeren Kaufverbindung der Möbel beiträgt, nicht abzuleiten. Grundlage der Annahme sind jedoch die Daten von Kundenkarteninhabern, die eher Intensivkunden der untersuchten Geschäftsstätte sind. Wettbewerbseffekte sind daher zwangsläufig schwächer ausgeprägt als bei der Gesamtkundschaft, so daß für eine Analyse dieser Einflußgrößen Kundenkarteninformationen weniger geeignet sind.

\section{Der Nachfrageverbund als zeitraumbezogener Ansatz der Verbundmessung}

Die Erfassung von Verbundbeziehungen ist auch losgelöst vom Kaufzeitpunkt durchführbar. Dabei löst sich die Verbundmessung von den bisherigen Vorgehensweisen. 12 statt die Käufe vom Konsumenten loszulösen und ohne Bezug zum Konsumenten in eine Häufigkeitsmatrix einzutragen, wird hierbei für jeden Kunden ein Datensatz erstellt, der seine relativen Kaufintensitäten für jede Abteilung zusätzlich zu seinen demographischen Daten enthält.

Vgl. Kapitel B.2.1 dieser Untersuchung.

12 Vgl. Kapitel B.1 dieser Arbeit. 
Die weitergehende Analyse dieser Datensätze erfolgt zweistufig. Zum einen werden Abteilungsgruppen mit Hilfe der Faktorenanalyse gebildet. Diese Faktoren umfassen Abteilungen, in denen die Konsumenten gleiche Kaufintensitäten aufweisen. Diese Information ist z.B. für die Zusammenstellung von Werbeware innerhalb einer Werbemaßnahme erforderlich, da für in einem Faktor enthaltene Abteilungen ein gleiches Kaufinteresse vorliegt. Ob diese Faktoren innerhalb verschiedener demographischer Gruppen stabil sind, gibt Hinweise auf die Anwendbarkeit bestimmter Zusammenstellungen für alle Altersgruppen.

Das Vorliegen eines Faktors und damit einer Abteilungskombination innerhalb einer demographischen Gruppe allein ergibt jedoch noch keinen Hinweis, ob die Werbewarenzusammenstellung in dieser Gruppe beworben werden sollte. Gleiche Intensitäten bedeuten nicht zwangsläufig ein hohes Intensitätsniveau. Daher muß in einem zusätzlichen schritt das Intensitätsniveau für die Faktoren innerhalb der Gruppen ermittelt werden, da nur bei hohem Intensitätsniveau ein hohes Interesse $z u$ unterstellen ist. Somit ergibt diese zweite stufe nicht die Information "was zusammen beworben wird" sondern "wer die Werbung erhalten soll".

\subsection{Verbundgruppenbildung in der Gesamtstichprobe}

Der Nachfrageverbund als zeitraumbezogenes Konstrukt erfaßt die Kaufintensitäten jedes Kunden für jede Abteilung. Durch eine faktorenanalytische Verdichtung wird erreicht, daß Gemeinsamkeiten im intensitätsbezogenen Kaufverhalten in unterschiedlichen Gruppen zusammengefaßt werden. Daraus ist abzuleiten, daß diese Abteilungsgruppierungen allgemeine Bündel bzw. Verbundgruppen von Kaufintensitäten im zeitablauf darstellen. Das Kaufverhalten in einer Abteilung, die eine hohe Ladung bzgl. der Faktoren aufweist, ist somit ty- 
pisch für das Kaufverhalten in anderen Abteilungen, die ebenfalls hoch auf diesen Faktor laden. Auf diese Weise ist es möglich, Bedarfsgruppen zu bilden, die das intensitätsmäßige Kaufverhalten von Konsumenten abbilden können.

Um diese Gruppen bilden zu können, wurde anhand einer Faktorenanalyse eine Verdichtung der Kaufintensitätsvariablen vorgenommen. Insgesamt ergab sich als Resultat eine Verdichtung auf 14 Faktoren nach dem Kaiser-Kriterium, bei dem nur Faktoren mit einem Eigenwert größer als 1 berücksichtigt werden. Der 14. Faktor besitzt allerdings mit Abstand den geringsten Eigenwert. ${ }^{13}$ Aufgrund seines geringen Erklärungsanteils wird er nicht als eigenständiger Faktor einbezogen. Eine Übersicht über die ermittelten Faktoren mit ihren bestimmenden Abteilungen gibt Abbildung 23. Für eine bessere Interpretierbarkeit der Faktorenstruktur wurde eine VarimaxRotation durchgeführt. ${ }^{14}$ Als Extraktionsverfahren wurde die Hauptkomponentenanalyse gewählt. Anhand der hohen Faktorladungen ${ }^{15}$ war es möglich, eindeutige und plausible zusammen-

13 Der Eigenwert beträgt 1,023. Der nächst höhere wert liegt bei 1,39 .

14 Vgl. zum Verfahren der Faktorenanalyse z.B. Backhaus, K. et al., Multivariate Analysemethoden, a.a.0., S. $67 \mathrm{ff}$; Meffert, H., Marktforschung, a.a.o., s. 89 ff. und speziell zum Verbund auch Kapitel B.1.241 dieser Untersuchung.

15 Die höchsten Ladungen je Faktor lagen zwischen 0,6 und 0,9 . Als Kernladungen, die den Faktor maßgebend bestimmen, werden Faktoren bis $z u$ einer Ladung von mindestens 0,5 aufgefaßt. Zur interpretatorischen Abrundung der sortimentsbezogenen Inhalte eines Faktors werden z.T. Faktoren mit einer Ladung von mindestens 0,3, in Ausnahmefällen auch 0,25 herangezogen. Sie werden als Randsortimente eines Faktors bezeichnet. Diese Ladungen werden berücksichtigt, weil sie trotz ihrer geringen absoluten Ausprägung relativ stark sind. Der überwiegende Teil aller Ladungswerte liegt ansonsten zwischen 0 und 0,15 . Einen vollständigen Überblick über Faktoren und Ladungen der bestimmenden Abteilung gibt Abbildung 23. 


\begin{tabular}{|c|c|c|c|c|c|}
\hline \multicolumn{2}{|c|}{ FAKTOR 1 "Kinderbedarf" } & \multicolumn{2}{|c|}{ FAKTOR 2 "Damenmode" } & \multicolumn{2}{|c|}{ FAKTOR 3 "Grundbedarf" } \\
\hline Vorschulkleidung & 0,843 & Damen-Wollwaren & 0,769 & Lebensmittel & 0,772 \\
\hline Kinder-Wollwaren & 0,795 & Röcke & 0,750 & Waschen/Reinigen & 0,770 \\
\hline Kinderwäsche & 0.781 & Blusen & 0,715 & Körperpflege & 0,714 \\
\hline Kinderstrümpfo & 0.762 & Damenschuhe & 0,574 & Kosmetik & 0,512 \\
\hline Kinderschuhe & 0,659 & Jeans/Freizeit & 0.556 & Damenstrümpfe & 0,493 \\
\hline Spielwaren & 0,559 & Kleider & 0,541 & Schreibwaren & 0.462 \\
\hline Teenagerbekleidung & 0,551 & Kostūme/Kombimode & 0,522 & Restaurant & 0,438 \\
\hline \multirow[t]{2}{*}{ Babyartikel } & 0,365 & Damenhosen & 0,459 & & \\
\hline & & Da-Mäntel (Leder etc.) & 0,410 & & \\
\hline \multicolumn{2}{|c|}{ FAKTOR 4 "Hobby/High-Tech" } & \multicolumn{2}{|c|}{ FAKTOR 5 "Damenwäsche" } & \multicolumn{2}{|c|}{ FAKTOR 6 "Herrenkleidung" } \\
\hline Radio/TV/Video & 0,679 & Miederwaren & 0,681 & Herrenartikel & 0,663 \\
\hline Computer/Bürotechnik & 0,607 & Damen-Tageswäsche & 0,664 & Herren-Trikotagen & 0,579 \\
\hline Schallplatten/Tontr. & 0,567 & Damen-Nachtwäsche & 0,535 & Herren-Wollwaren & 0,574 \\
\hline Photo/Optik & 0,551 & Damenstrūmpfe & 0,494 & Anzüge/Hosen & 0,570 \\
\hline Bücher/Zeitschriften & 0,526 & Kosmetik & 0,380 & Herrenstrümpfe & 0,523 \\
\hline Schreibwaren & 0,437 & Kostüme/Kombimode & 0,332 & Men's Fashion & 0,508 \\
\hline Heimwerker/Autozub. & 0,426 & Haushalt-/Bettwäsche & 0,329 & He-Mäntel (Leder etc.) & 0,353 \\
\hline Lampen & 0,422 & Körperpflege & 0,308 & Jeans/Freizeit & 0,274 \\
\hline Elektro & 0,391 & Modeaccessoires & 0,301 & Herrenschuhe & 0,253 \\
\hline Spielwaren & 0,355 & & & & \\
\hline \multirow{2}{*}{\multicolumn{2}{|c|}{ FAKTOR 7 "Haush.ausstatt." }} & \multicolumn{2}{|c|}{ EAKTOR 8 "Selbst-Schneidern" } & \multicolumn{2}{|c|}{ "Sport" } \\
\hline & & Kloiderstoffe & 0,889 & Sportbekleidung & 0,639 \\
\hline Rund u.d.Küche & 0,556 & Kurzwaren & 0,855 & Sportartikel & 0,625 \\
\hline Geschenkartikel & 0,513 & Handstrickgarne & 0,304 & Wintersport & 0,570 \\
\hline Haushalt-/Bettwäsche & 0,407 & & & Sommersport/Strand-/ & 0,430 \\
\hline Handstrickgarne & 0,328 & & & Badezubehör & \\
\hline Alles für's Bad & 0,324 & & & & \\
\hline \multicolumn{2}{|c|}{ FAKTOR 10 "Einrichtung" } & \multicolumn{2}{|c|}{ EAKTOR 11 "Ālterer Herr" } & \multicolumn{2}{|c|}{ EAKTOR 12 "Da-Accessoires" } \\
\hline Möbel & 0,626 & Herrenschuhe & 0,607 & Damenhüte & 0,677 \\
\hline Teppiche & 0,535 & He-Māntel (Leder etc.) & 0,483 & Handschuhe & 0,490 \\
\hline Gardinen & 0,493 & Anzüge/Hosen & 0,438 & Modeaccessoires & 0,369 \\
\hline Bettwaren & 0,424 & Hausschuhe & 0,360 & Hausschuhe & 0,308 \\
\hline Alles für's Bad & 0,385 & Jeans/Freizeit & $-0,269$ & Da-mäntel (Leder etc.) & 0,276 \\
\hline Haushalt-/Bettwäsche & 0,296 & & & & \\
\hline Lampen & 0,251 & & & & \\
\hline \multicolumn{2}{|c|}{ EAKTOR 13 "Schlecht. Wetter" } & & & & \\
\hline Restaurant & 0,587 & & & & \\
\hline Schirme & 0,549 & & & & \\
\hline Lederwaren & 0,398 & & & & \\
\hline Uhren/Schmuck & 0,248 & & & & \\
\hline
\end{tabular}

Abb. 23: Faktoren mit ihren bestimmenden Abteilungen in der Grundgesamtheit 
hänge zwischen den Kaufintensitäten in verschiedenen Abteilungen aufzudecken. Der erklärte Varianzanteil lag bei $71,3 \%$. Aufgrund der sortimentsbezogenen Inhalte konnten den einzelnen Faktoren sogar thematische Überschriften zugeordnet werden, die ein bestimmtes bedarfsorientiertes situatives oder lebensabschnittabhängiges Raufintensitätsbündel widerspiegeln.

Bei der Faktorinterpretation tritt ein weiteres Phänomen auf. Es existieren Abteilungen, die auf verschiedene Faktoren hoch laden (z.B. die Abteilung Anzüge/Hosen auf Faktor 6 und Faktor 11). Dies bedingt aber nicht, wie sonst bei Faktorenanalysen, interpretatorische Probleme. Es spiegelt vielmehr die Breite des Sortiments wider. So kann eine Abteilung z.B. Sortimentsbestandteile für unterschiedliche Altersgruppen und damit für andere Bedarfsbilder enthalten. 16

Im einzelnen ergaben sich die folgenden Nachfrageverbundgruppen:

- Kinderbedarf

- Damenmode

- Grundversorgung Haushalt

- Hobby incl. High-Tech

- Damenwäsche

- Herrenkleidung (Tendenz: jüngerer Herr)

- Haushaltsausstattung

- Selbst-Schneidern/Kleidung anfertigen

- Sport.

- Einrichtung

- Herrenkleidung (älterer Herr)

- Damenmode-Accessoires (elegante Dame)

- "Schlechtes Wetter"/Shopping. 


\section{Verbundgruppe "Kinderbedarf":}

Die Abteilung mit dem höchsten Ladungswert für diesen Faktor ist der Bereich Vorschulkinder- und Kinderkleidung. Zu dieser eher oberbekleidungsorientierten Abteilung treten offensichtlich komplementäre sortimentsbereiche mit Kinderwollwaren, Kinderwäsche, Kinderstrümpfen und Kinderschuhen. All diese Sortimentsbereiche weisen noch Ladungswerte über 0,76 auf, bis auf die schuhe mit 0,66.17 $\mathrm{zu}$ diesen sortimenten treten ebenfalls noch mit starken Ladungen über 0,55 die spielwaren und die Teenager-Bekleidung. Als Randsortimente kommen Baby-Artikel $z u$ dieser Gruppe. Insgesamt spiegelt dieser Faktor eine Ausrichtung der Kaufintensität von Konsumenten auf den Kinderbereich wider.

\section{Verbundgruppe "Damenmode":}

Dieser Faktor umfaßt vorwiegend die klassischen Damenoberbekleidungssortimente mit den Bereichen Damen-Wollwaren, Röcke und Blusen als dominierende Abteilungen. Ebenfalls sehr starke Ladungen weisen die Abteilungen Junge Mode mit Jeans und Freizeitmode sowie Kleider und die Abteilung Jacken/ Kombinationsmode/Kostüme auf. Aufgrund der Tatsache, daß in der heutigen Zeit auch tendenziell ältere Damen Bekleidung aus dem Jeans- und Freizeitmodebereich tragen, stellt diese Abteilung keinen Fremdkörper innerhalb dieser Verbundstruktur dar.

Als Randsortimente mit relativ starker Anbindung sind noch die Sortimentsbereiche Damenhosen und Damenmäntel mit Lederund Pelzmänteln mit diesem Faktor verbunden. Die relativ schwache Anbindung dieser Bereiche läßt sich zum einen dadurch erklären, daß Hosen häufig aus dem Jeans-Bereich stam-

17 Dies könnte an einer allgemeinen Dominanz des Facheinzelhandels im Bereich Schuhe liegen, da bei Damenbekleidungssortimenten nur eine Ladung von 0,57 und bei den Herren sogar nur 0,27 in den jeweiligen Schuhsegmenten erreicht werden. 
men, wenn sie von Damen getragen werden. Zum anderen dürfte sich der allgemeine Trend der Abkehr vor allem von Pelzwaren auf die Kaufintensität im Bereich Damenmäntel auswirken.

\section{Verbundgruppe "Grundversorgung Haushalt":}

In diesem Faktor sind überwiegend Abteilungen zusammengefaßt, die zur Grundlage einer Haushaltsführung gehören. Als zentrale Sortimentsbereiche stellen sich in diesem Zusammenhang die Abteilungen Lebensmittel, Waschmittel, Raum- und Tierpflege sowie Körperpflege dar. Eine starke Ladung weist darüber hinaus die Gebrauchskosmetik auf. Der Grundbedarf wird normalerweise nicht in einem Vollsortimenter in Citylage intensiv gedeckt. Daher zeigt sich hier eher das Bild eines Konsumenten, der häufig unter Zeitdruck z.B. auf dem Heimweg von der Arbeitsstätte seinen Bedarf deckt. Als Randsortimente mit $z . T$. relativ starker Ladung nahe 0,5 kommen noch die Abteilungen Damenstrümpfe, Schreibwaren, Restaurant und Rund um die Küche zu diesem Faktor.

\section{Verbundgruppe "Hobby incl. High-Tech":}

In dieser Gruppe fließen grundsätzlich Freizeitsortimente zu einem Faktor zusammen. Dies zeigt sich bei der gesamten Palette von den zentralen Abteilungen bis hin zu den Randsortimenten. Als zentraler Bereich ist dabei die High-Tech-orientierte Freizeitgestaltung zu sehen. Dazu gehören die Abteilungen Radio/Fernsehen/Video, Computer/Bürotechnik und Photo/Optik, die die höchsten Ladungswerte aufweisen. Eine starke Ladung weist auch die Abteilung Bücher/Zeitschriften auf. Als Randsortimente, die eher auf den Do-it-yourself und Bastlerbereich zielen, sind die Abteilungen Schreibwaren, Heimwerker/Autozubehör, Lampen, Elektro- und spielwaren vertreten. Insgesamt ergibt sich das Bild einer komplexen Freizeitabdeckung. 


\section{Verbundgruppe "Damenwäsche":}

Die Abteilungen mit den stärksten Ladungen innerhalb dieses Faktors sind die Bereiche Miederwaren, Damen-Tageswäsche und Damen-Nachtwäsche. Erweitert um die Fülle von Randsortimenten mit z.T. starken Ladungswerten ergibt sich darüber hinaus das Bild einer Dame, die insgesamt sehr viel Wert auf gepflegtes Aussehen, z.B. als Vorbereitung auf Abendunterhaltungen wie Theaterbesuche, Tanzen etc. legt. $\mathrm{Zu}$ diesen Randsortimenten zählen die Abteilungen Damenstrümpfe, Kosmetik, Jacken/Kombinationsmode/Kostüme sowie Haushalts- und Bettwäsche, Körperpflege und Modeaccessoires.

\section{Verbundgruppe "Herrenkleidung":}

Diese Gruppierung umfaßt die komplette spannweite der Herrenbekleidung von den Wäschebereichen bis zum Mantel und vom Anzug bis zur Freizeitkleidung, so daß diese Gruppierung Kaufintensitäten widerspiegeln, die eine komplette Einkleidung eines männlichen Konsumenten repräsentieren. Der Bereich von Abteilungen mit starker Ladung umfaßt mit 6 Abteilungen eine große Anzahl die nur in der Damenmode-Gruppe mit 7 Abteilungen noch höher ist. $\mathrm{Zu}$ diesen Abteilungen mit hoher Ladung zählen die Herrenartikel, die Herren-Trikotagen, Herren-Wollwaren, Anzüge-Hosen, Herren-Strümpfe und Men's Fashion mit eher hochwertiger oberbekleidung. Als Randsortimente treten dazu noch die Herrenmäntel, die junge Mode mit Jeans und Freizeit sowie die Herrenschuhe. Auffällig ist dabei, daß im Herrenmodebereich ebenso wie bei der Damenmode der Bereich Junge Mode mit Jeans und Freizeitmode verbunden ist. Wenn auch die Ladung dieser Abteilung bei der Damenmode ungleich höher ist, so kann doch festgestellt werden, daß im Bereich Freizeit nicht unbedingt eine geschlechtsbezogene Trennung von Sortimenten in verschiedene Abteilungen stattfinden muß. 


\section{Verbundgruppe "Haushaltsausstattung":}

Dieser Faktor repräsentiert ein Kaufmuster, das vornehmlich auf die Ausgestaltung eines Haushalts ausgelegt ist. Da von seiten des Handelsunternehmens bereits eine zusammenführung kleiner Abteilungen $z u$ größeren vorgenommen wurde, bilden relativ wenige Abteilungen die Bestimmungsfaktoren für diese Gruppierung. Mit starker Ladung sind dies die Abteilungen Rund um den gedeckten Tisch, Rund um die Küche und Geschenkartikel. Als Randsortimente sind darüber hinaus die Abteilungen Haushalts- und Bettwäsche, Handstrickgarne und Badezimmerzubehör vertreten. Aus den Abteilungsbezeichnungen ist ablesbar, daß bereits Ausstattungsbereiche nach Wohnbereichen zusammengefaßt wurden.

\section{Verbundgruppe "selbst-schneidern":}

Dieser Faktor repräsentiert eine reine spezialgruppe, die auch nur zwei bestimmte Abteilungen und ein Randsortiment aufweist. Allerdings weisen die bestimmenden Abteilungen mit einer Ladung über 0,85 extrem hohe Werte auf. Diesen Abteilungen sind Kleiderstoffe und Kurzwaren zugeordnet. Als Randsortiment treten noch Handstrickgarne dazu. Insgesamt zeigt dieser Faktor das Bild eines Do-it-yourself-Konsumenten im Bekleidungsbereich.

\section{Verbundgruppe "sport":}

Auch dieser Faktor weist relativ wenige Abteilungen mit hoher Ladung und wenig Randsortimente auf. Dies kann auch davon abhängen, daß im Datensatz z.B. Sommersport und strandmoden eine gemeinsame Abteilungsnummer aufweisen. Dadurch ist möglicherweise der Randsortimentwert von $0,43 \mathrm{zu}$ erklären. Als bestimmende Abteilungen dieses Faktors sind die Bereiche sportbekleidung, sportartikel und wintersporttextilien anzuführen, wobei sommersporttextilien und strandmoden ein Randsortiment bilden. Insgesamt sind alle Abteilungen auf einen aktiven, freizeitorientierten Konsumenten ausgerichtet. 


\section{Verbundgruppe "Einrichtung":}

Dieser Faktor vereinigt Abteilungen, die auf die Einrichtung eines Haushalts bezogen sind. Als Abteilungen mit starken Ladungen sind Möbel und Teppiche $z u$ verzeichnen. Dazu ist anzumerken, daß im Rahmen dieser Untersuchung verschiedene Möbelabteilungen $z u$ einem Bereich zusammengefaßt worden sind. Bei diesem Faktor sind auch Randsortimente mit z.T. relativ starken Ladungen $z u$ verzeichnen. Auch diese runden das Bild eines einrichtungsorientierten Faktors ab. Dazu gehören die Gardinen $(0,49)$, Bettwaren $(0,42)$, Badezimmerzubehör $(0,39)$, Haushalts- und Bettwäsche sowie Lampen.

\section{Verbundgruppe "Herrenkleidung (älterer Herr)":}

In diesem Faktor vereinigen sich wiederum Kaufintensitäten aus dem Bereich Herrenkleidung. Allerdings scheint hier eine Ausrichtung auf den Bedarf älterer Herren offensichtlich. Dies wird vor allem durch die negative Ladung der Abteilung Junge Mode und Jeans- und Freizeitmode in Höhe einer Randsortimenteladung unterstrichen. Auch das Randsortiment Hausschuhe scheint in diese Richtung $\mathrm{zu}$ deuten. Insgesamt laden die Abteilungen Herrenschuhe, Herrenmäntel, Anzüge/Hosen, Hausschuhe und Heimwerker positiv auf diesen Faktor, wobei auch hier eher seriöse sortimentsbereiche, die auf ein tendenziell älteres Publikum gerichtet sind, im Vordergrund stehen.

\section{Verbundgruppe "Damenmode/Accessoires":}

Auch im Bereich der Damenmode ergibt sich mit diesem Faktor wie im Herrenbereich eine zweite Bekleidungsgruppe. Ebenso ist in diesem Zusammenhang eher eine Ausrichtung auf Bekleidungselemente für ältere Damen bzw. elegante kleidung vor allem aus dem Accessoiresbereich festzustellen. Als Abteilung mit höherer Ladung für diesen Faktor sind nur Damenhüte vorhanden. Allerdings weisen die Randsortimente z.T. relativ hohe Ladungen auf. Die Randsortimente bestehen aus Handschu- 
hen $(0,49)$, Modeaccessoires $(0,37)$, Hausschuhen und Damenmänteln. Auch die Abteilung Damenschuhe weist eine, wenn auch mit 0,26 schwache Bindung an diesen ermittelten Bekleidungsfaktor auf.

\section{Verbundgruppe "schlechtes Wetter/shopping":}

Auch diesem Faktor kann ein komplexes Konsumentenbild zugeordnet werden. Es zeigt einen Konsumenten mit zeit zum Einkaufen, was vor allem aus der dominierenden stellung des Restaurants abzulesen ist. Gleichzeitig zeigt die orientierung an hochwertigen Sortimenten wie Uhren/Schmuck/Juwelierwaren, Damenmäntel mit Leder- und Pelzmoden ein gewisses finanzielles Potential. Tendenziell dürfte dabei das Kaufverhalten älterer Konsumenten erfaßt werden.

Als Abteilungen mit hoher Ladung sind hier die Bereiche Restaurant und Schirme (evtl. ein Schlechtwetterbesuch) zu verzeichnen. Darüber hinaus treten die Abteilungen Lederwaren, Uhren/Schmuck (Juwelierwaren), Damenmäntel mit Pelzund Ledermoden sowie Handschuhe als Randsortimente mit zum Teil allerdings relativ schwachen Ladungswerten auf.

Insgesamt ergibt sich bei der Faktorenbildung ein deutliches System plausibler Kaufverhaltensgruppen, die jeweils ein situations- bzw. lebensabschnittsorientiertes Konsumentenbild beinhalten. Damit wird auch deutlich, wie sinnvoll eine Kundensegmentierung anhand dieser Faktoren als Grundlage einer clusteranalyse zur Zielgruppenbildung ist. Dabei lassen sich dann durch eine Betrachtung der Ausprägungen der Faktoren in den zielgruppen, aber auch aus einem Vergleich mit soziodemographischen Größen, wertvolle Aufschlüsse für eine segmentspezifische Marktbearbeitung gewinnen. 18 


\subsection{Verbundintensität und sortimentsgruppenbildung in aus- gewählten sozioökonomischen zielgruppen}

Die Untersuchung der Intensitäten und Faktorenstrukturen innerhalb der unterschiedlichen Alters- und Einkommensgruppen dient zur Überprüfung der Arbeitshypothese zum Nachfrageverbund $\mathbf{H}_{\mathbf{N R}}$ II:

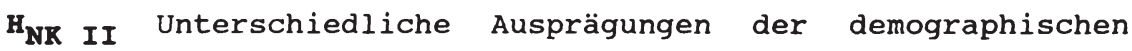
Variablen "Alter" und "Einkommen" haben keine Auswirkungen auf die grundsätzliche struktur der mit der Nachfrageverbundanalyse ermittelten Bedarfsgruppen. Lediglich die Kaufintensitäten dieser Gruppen variieren.

\subsection{Analyse der abteilungsbezogenen Intensitätswerte}

Bei der Betrachtung der durchschnittlichen Kaufintensitäten verschiedener Abteilungen in den jeweiligen Untergruppen zeigt sich, daß die unterschiedlichen Altersgruppen zu einer wesentlich stärkeren Polarisierung der Werte führen als die Einkommensgruppen. Dies kann einerseits damit zusammenhängen, daß Altersklassen wesentlich deutlicher einzelne Lebensphasen kennzeichnen können als das Einkommen. Unterschiedlichen Lebensphasen liegen auch klar unterschiedliche Bedürfnisse zugrunde, die sich in den Kaufintensitäten widerspiegeln. Dabei ist zwar zu berücksichtigen, daß die drei gebildeten Altersklassen zwar relativ grob sind, aber trotzdem $z u$ einer klaren Differenzierung der Ergebnisse führen.

Ein zentrales Argument im Bereich der Differenzierung anhand des Einkommens ${ }^{19}$ ist die fehlende Preislagendifferenzierung

19 Das Einkommen ist als demographische Angabe im Rahmen dieser Untersuchung dem Antragsbogen des jeweiligen Kartenbesitzers entnommen. Diese Größe wird vom kartenausMarcus Poggenpohl - 978-3-631-75099-5 
der vorliegenden Daten auf Abteilungsebene. Für diesen Aspekt sprechen auch die wenigen Werte, die in verschiedenen Einkommensbereichen ansatzweise eine Polarisierung zeigen. Dabei ist festzustellen, daß in Abteilungen mit grundsätzlich eher hochwertigen, nicht dem Grundbedarf zuzurechnenden Waren am ehesten Divergenzen vorliegen. So sind die schwächsten Abteilungen im Bereich bis 36.000 DM Einkommen die Abteilungen Bürotechnik/Computer $(0,65)$, Herrenmäntel/Leder $(0,67)$, Sportartikel $(0,74)$, Photo/Optik $(0,75)$, Lampen $(0,76)$. Allgemein schwach ist eher der Kinderbekleidungsbereich sowie der Herrenbekleidungsbereich (ca. 0,8 bis 0,95), wohingegen die Damenmode $z . B$. Kleider $(1,25)$, Hüte $(1,29)$ knapp oberhalb eines Intensitätswertes von 1 liegt.

Quasi das Spiegelbild dieser Gruppe ist die Einkommensklasse über 80.000 DM mit hohen werten im Bereich sport, z.B. Sportartikel $(1,39)$, Sportbekleidung $(1,25)$, Lampen $(1,28)$, Kinderbekleidung z.B. Kinderstrümpfe $(1,42)$, Kinderwäsche $(1,30)$. Dagegen zeigen sich relativ geringe werte im Damenmodebereich z.B. Kleider $(0,52)$ aber auch bei Men's Fashion $(0,77)$. Dieser starke Effekt innerhalb des Bekleidungsbereichs liegt möglicherweise im sortiment begründet, da diese Einkommensgruppe häufig sehr hochwertig in Fachgeschäften (z.B. Boutiquen) einkauft.

In den beiden mittleren Einkommensgruppen pendeln sich fast alle Intensitätswerte nahe bei 1 ein (ca. 0,85 bis 1,1 ). Im Einkommensbereich von 36.000 DM bis 50.000 DM gibt es zwei relativ hohe Werte im Damenmodebereich mit 1,28, bei Damenmänteln mit Leder und Pelzen sowie bei den Röcken mit 1,2. Bei der Einkommensgruppe mit 51.000 DM bis 80.000 DM Jahres-

gebenden Unternehmen nicht überprüft, so daß sich Unschärfen bilden können. Dabei können von seiten des Antragsstellers z.B. Prestigegründe für eine falsche Angabe vorliegen. Darüber hinaus ist der im Kartenantrag aufgeführte Begriff "Netto-Haushaltseinkommen" eventuell für einige Antragsausfüller nicht klar greifbar, so daB es zu unbeabsichtigten Fehleinschätzungen kommt. 
einkommen zeichnen sich ebenfalls zwei Werte ab, die außerhalb des normalen Rahmens liegen. Dies sind Computer/Bürotechnik mit 1,34 und Photo/Optik mit 1,22. Auch darin spiegelt sich wider, daß die höheren Einkommen eher im Bereich der hochwertigen Sortimente hohe Kaufintensitäten aufweisen.

Im Gegensatz zu den Einkommensklassen bilden die Altersgruppen wesentlich komplexere Kaufmuster mit zugleich stärker polarisierten werten ab. Die ermittelten Kaufintensitäten spiegeln klar unterschiedliche Bedarfsmuster der Gruppen in verschiedenen Lebensphasen wider.

So zeigen die Intensitätswerte der Altersgruppe von 18-35 Jahren die Bedürfnisse einer jungen Familie. Der gesamte Damenmodebereich weist mit Werten zwischen 0,43 (Hüte) und 0,57 (Röcke) sehr niedrige Werte auf. Ähnlich verhält es sich im Herrenmodebereich, auch wenn dort die Werte bei ca. $0,8-0,9$ liegen. Aus dem gesamten Bekleidungsgereich weisen nur die Abteilungen Anzüge/Hosen $(1,01)$, Men's Fashion $(1,20)$ und Junge Mode mit Jeans und Markenshops $(1,23)$ überdurchschnittliche Intensitätswerte auf. Auch der gesamte Sportbereich und der Wäschebereich sowohl im Damen als auch im Herrenbereich (ca. $0,7-0,8)$ sowie der gesamte Einrichtungsbereich $(0,5-0,8)$ mit 0,29 bei Teppichen zeigt unterdurchschnittliche Werte.

Eine überdurchschnittliche Warengruppe ist im Bereich des Baby- und Kleinkinderbedarfs zu finden mit spitzenwerten von 1,75 in der Abteilung Babyartikel und 1,51 bei den Kinderschuhen. Entsprechend hoch ist auch der wert bei Spielwaren $(1,27)$. Es ergeben sich also in einem umfangreichen Feld sehr niedriger Intensitätswerte zwei klare Akzente. Zum einen Junge Mode im Freizeitbereich und die "Einsteigermode" für Berufsanfänger in nicht handwerklichen Berufen, zum anderen der Baby- und Kleinkinderbereich. Dazu tritt noch ein 
dritter Aspekt, der mit Freizeit und "High Tech" zu betiteln ist mit den Abteilungen Schallplatten/Tonträger $(1,41)$, Computer $(1,25)$ und Radio/Fernsehen/Video $(1,05)$.

Ein weiterentwickeltes Bild des Familienbedarfs ergibt sich in der Altersgruppe von 36-50 Jahren. Die Kaufintensitätswerte sind vor allem in den sehr schwachen Gruppen der Altersgruppe bis 35 allgemein höher und liegen vielfach nahe bei 1. So liegt der gesamte Damenmodebereich zwischen 0,92 und 1,17. Noch immer ist der Bereich Junge Mode mit Jeans und Markenshops mit 1,22 die Abteilung mit dem höchsten Intensitätswert. Der gesamte Wäschebereich sowohl bei den Damen als auch bei den Herren liegt knapp oberhalb des Durchschnittswertes von 1. Ebenso entwickeln sich die werte bei der Herrenmode mit einem Spitzenwert von 1,27 bei der Men's Fashion.

Auch der Einrichtungsbereich weist nun höhere, jedoch noch knapp unterhalb von 1 liegende werte auf. Der sportbereich zeigt durchgängig Werte oberhalb von 1,1 .

Besonders hervorzuheben ist die Entwicklung im Bereich der Kindermode. Hier ergibt sich eine Verschiebung von Baby- und Kleinkinderbedarf zur Kinder- und Jugendlichenmode. Insgesamt zeigt sich also das Bild einer sich etablierenden Familie mit Kindern im Jugendalter, wobei sich die wirtschaftliche situation soweit entwickelt hat, daß auch Ausgaben im Einrichtungsbereich und bei hochwertigen waren (Uhren/ Schmuck mit 1,1 etc.) möglich sind.

Auch die Kaufintensitäten der Gruppe der über 50jährigen entwickeln sich plausibel weiter. Es sind wiederum klare Sortimentsbereichsreaktionen $z u$ erkennen. Der gesamte Damenmodebereich weist stark überdurchschnittliche Kaufintensitäten auf. Nur der Bereich Junge Mode liegt stark unter dem Durchschnitt $(0,55)$. Der Herrenmodebereich weist bis auf die 
Mäntel $(1,01)$ knapp unterdurchschnittliche Werte auf, wohingegen der gesamte Wäschebereich knapp oberhalb des Durchschnitts liegt. Der Kindermodebereich zeigt sich hier stark unterdurchschnittlich, wohingegen der Einrichtungsbereich durchgängig Werte weit oberhalb von 1 aufweist (Spitzenwert 1,55 bei Teppichen).

\subsection{Faktorenbildung in ausgewählten Altersklassen}

Bei der Auswertung der Faktorenbildung in den unterschiedlichen Altersklassen fällt auf, daß einerseits viele Abteilungskombinationen mit den bei der Gesamtanalyse erhaltenen Faktorengrößen Ähnlichkeiten aufweisen. Abbildung 24 zeigt einen Überblick über Gemeinsamkeiten in der struktur der Faktoren in Untergruppen und Grundgesamtheit. Somit lassen sich auch in den einzelnen Altersklassen den einzelnen Faktoren relativ komplexe Bilder von Kaufbedürfnissen bestimmter Personengruppen bzw. unterschiedliche Kaufintentionen zuordnen. Andererseits finden sich auch die bei der Analyse der Kaufintensitäten in den Altersklassen zusammengefaßten Gruppen wieder, so daß insgesamt zwei Einflußfaktoren gegeben sind, die zur Interpretation der Faktoren herangezogen werden können.

Die Analyse der Faktorenbildung in den Untergruppen ist zum einen notwendig, um die grundsätzlichen Bedarfsgruppen für die jeweiligen Alters- und Einkommensklassen herauszuarbeiten. Zum anderen besteht eine wichtige Aufgabe der folgenden Analyse aber auch darin, die stabilität der für die Grundgesamtheit ermittelten Bedarfsgruppen bzw. Faktoren bzgl. der Abteilungskombinationen in den Untergruppen $z u$ überprüfen.

Dazu ist es unerläßlich, jeden analysierten Faktor (je Untergruppe zwischen 16 und 19 Faktoren) vor allem mit den Faktoren der Grundgesamtheit zu vergleichen. 


\begin{tabular}{|c|c|c|c|c|c|c|c|c|}
\hline \multicolumn{2}{|c|}{ GRUNDGESAMTHEIT } & \multicolumn{3}{|c|}{ ALTERSGRUPPEN (JAHRE) } & \multicolumn{4}{|c|}{ EINKOMMENSGRUPPEN (TOM) } \\
\hline \multirow{2}{*}{$\begin{array}{l}\text { FAKTORNAME } \\
\text { KINDERBEDARF }\end{array}$} & \multirow{2}{*}{$\begin{array}{c}\text { FAKTOR- } \\
\text { NUMMER } \\
1 \\
\end{array}$} & \multirow{2}{*}{$\begin{array}{l}\text { BIS } 35 \\
34\end{array}$} & $36 \cdot 50$ & | ÜBER 50 & BIS 36 & $36 \cdot 50$ & $50 \cdot 80$ & | ŨBER 80 \\
\hline & & & 14 & $\mathbf{T}$ & $\hat{t}$ & 4 & $\boldsymbol{1}$ & 2 \\
\hline DAMENKLEIDUNG & 2 & $\rightarrow$ & $\rightarrow$ & $2 \rightarrow$ & $\boldsymbol{t}$ & $\lambda$ & $3 \pi$ & $\rightarrow$ \\
\hline GRUNOBEDARF & 3 & $\pi$ & $\hat{1}$ & $\mathbf{T}$ & $1 \rightarrow$ & $\lambda$ & $\pi$ & $\rightarrow$ \\
\hline HOBBY/FREIZEIT & 4 & $\rightarrow$ & $\boldsymbol{t}$ & $\rightarrow$ & $\pi$ & 1 & $\uparrow$ & 10 \\
\hline DAMEN-WASCHE & 5 & 1 & $\pi$ & $y$ & $\pi$ & $\rightarrow$ & $\begin{array}{c}7 \\
16 \\
\end{array}$ & 4 \\
\hline HERRENKLEIOUNG & 6 & $\Rightarrow$ & $\mathbf{T}$ & $\lambda$ & $\pi$ & $\uparrow$ & $\boldsymbol{T}$ & $\lambda$ \\
\hline $\begin{array}{l}\text { HAUSHALT- } \\
\text { AUSSTATTUNG }\end{array}$ & 7 & $\rightarrow$ & $\mathbf{T}$ & $\mathbf{t}$ & 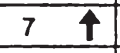 & $\lambda$ & $4 \pi$ & $\rightarrow$ \\
\hline SCHNEIDERN & 8 & $\uparrow$ & $\boldsymbol{T}$ & $\mathbf{T}$ & $10 T$ & 10 & $\mathbf{T}$ & 8 \\
\hline SPORT & 9 & 12 & $9 \pi$ & 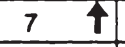 & $\begin{array}{rr}9 & \lambda \\
13\end{array}$ & $\pi$ & $\pi$ & $\rightarrow$ \\
\hline EINAICHTUNG & 10 & 19 & $10 \rightarrow$ & 1 & $12 \rightarrow$ & $\pi$ & $\lambda$ & 11 \\
\hline ALTEREA HERR & 11 & $(5)$ & (5) $y$ & 5 & (6) $\rightarrow$ & (4) & (5) $\quad y$ & (3) \\
\hline ALTERE DAME & 12 & 17 & $12 \pi$ & 1 & 54 & 13 & $11 \lambda$ & 16 \\
\hline SHOPPING & 13 & 16 & $13 \pi$ & 13 & $\begin{array}{l}17 \\
18\end{array}$ & 12 & $\downarrow$ & 17 \\
\hline $\begin{array}{l}\uparrow \text { NAHEZU IDENT } \\
\nearrow \text { SEHR ÄHNLIC } \\
\rightarrow \text { ZENTRALE ABT } \\
\downarrow \text { ÄHNLICHKEITE } \\
\downarrow \text { GLEICHE ZENT }\end{array}$ & $\begin{array}{l}\text { TISCHE AB } \\
\text { HE ABTEILU } \\
\text { TEILUNGSK } \\
\text { N VORHAN } \\
\text { TRALE ABTE }\end{array}$ & $\begin{array}{l}\text { BTEILUNGSKO } \\
\text { UNGSKOMBII } \\
\text { KOMBINATOI } \\
\text { NDEN; EINIGE } \\
\text { EILUNG; SON }\end{array}$ & $\begin{array}{l}\text { MBINATIONE } \\
\text { IATIONEN UN } \\
\text { IEN MIT HOH } \\
\text { ABTEILUNG } \\
\text { ST KAUM ÜB }\end{array}$ & $\begin{array}{l}\text { N UND WERT } \\
\text { ND WERTE } \\
\text { TER ÄHNLICHI } \\
\text { SKOMBINATIO } \\
\text { ERSCHNEIOU }\end{array}$ & $\begin{array}{l}\text { KEIT; ABWEIC } \\
\text { DNEN FINDEN } \\
\text { NGEN }\end{array}$ & $\begin{array}{l}\text { CHUNGEN IN } \\
\text { SICH WIEDE }\end{array}$ & $\begin{array}{l}\text { RANDSORTIM } \\
\text { R }\end{array}$ & MENTEN \\
\hline (ALLE BEWERTUNC & EN JEWEIL & LS IM V & ICH ZL & 政 & 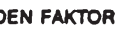 & & & \\
\hline
\end{tabular}

Abb. 24: Gemeinsamkeiten zwischen Faktoren in den Untergruppen und Faktoren der Grundgesamtheit

Abbildung 24 zeigt, daß es in jedem Fall Entsprechungen zwischen Faktoren der Grundgesamtheit und Faktoren in den Untergruppen gibt. Dies belegt bereits, wie stabil die bei der Gesamtstichprobe abgeleiteten Faktoren sind. Dies unterstreicht auch gleichzeitig die Eignung dieser Faktoren als clusterbildende Variablen zur segmentierung der Kartenkunden nach Verbundgrößen. 20

zunächst erfolgt jedoch eine detaillierte Analyse der Aussagen der Faktorenbildung in den spezifischen Untergruppen der 
Gesamtstichprobe. Als Beispiel sollen dafür die stets wiederkehrenden Faktoren der Damen- und Herrenkleidung oder des Kinder- und Einrichtungsbedarfs dienen. Diese Faktoren tauchen mit variierenden Ladungen z.B. zwischen Junger Mode und eher gesetzten Sortimenten immer wieder auf, wobei die Ladungsunterschiede sich durch die unterschiedlichen Grundbedürfnisse der Altersgruppen ergeben.

Zudem sagen die Ladungen nichts über Kaufintensitäten, sondern nur etwas über deren Ähnlichkeit innerhalb eines Faktors aus. Diese Aspekte erklären auch die Aufteilung der Bekleidungssortimente auf teilweise verschiedene Faktoren. Dabei werden die Abteilungen mit hoher Kaufintensität z.B. im Damenbekleidungssektor zusammengefaßt und die mit eher niedriger Kaufintensität in einem anderen Faktor. Falls nur eine Abteilung aus einer sinngruppe eine abweichende Intensität aufweist (z.B. Damenhüte oder Damenmäntel), kann diese in anderen Faktoren aufgehen, die ähnliche Kaufintensitäten aufweisen, aber sinngemä $B$ kaum $z u$ den anderen Abteilungen passen.

Auffällig ist darüber hinaus, daß z.T. relativ wenige Abteilungen für einen Faktor bestimmend sind, diese aber recht hohe Ladungswerte aufweisen (z.B. Faktor 9 in der Altersgruppe bis 36 Jahren, der als bestimmende Abteilungen nur Kleiderstoffe und Kurzwaren ausweist und der damit mit "Schneiderei" überschrieben wird). Dies zeigt, daß es insgesamt weniger bestimmende Abteilungen gibt als bei der $\mathrm{Ge}-$ samtanalyse. Vor dem Hintergrund, daß der Bedarf in den Einzelgruppen jedoch wesentlich spezifischer ist als in der Gesamtstichprobe, erscheint dies plausibel. Die Fallzahlen sind entsprechend der Alters- und Einkommensverteilung in den Gruppen unterschiedlich. ${ }^{21}$ Die geringste Fallzahl beträgt aber mit $8,4 \%$ der Stichprobe in der Altersklasse über 
65 Jahren 391. Dies gewährt einen ausreichenden Stichprobenumfang. Der erklärte Varianzanteil in den Untergruppen liegt zwischen $59,6 \%$ und $67,8 \%$

Bei der Analyse der Altersgruppe bis 35 Jahre ergeben sich 19 Faktoren nach dem Kaiser-Kriterium. Die Interpretation erfolgt anhand der Varimax-rotierten Faktorenmatrix. Da eine detaillierte Beschreibung aller Faktoren (insgesamt in allen Untergruppen mehr als 100) den Rahmen dieser Arbeit überschreitet, werden im folgenden zusammenfassende Aussagen für jede Untergruppe abgeleitet, die vereinzelt exemplarisch auf spezifische Werte verweisen.

In den ersten 14 Faktoren ergeben sich sowohl recht stabile Abteilungskombinationen als auch plausible Kaufmotive. Darüber hinaus lassen sich Überschneidungen mit den Faktoren, die für die Grundgesamtheit abgeleitet werden, feststellen. Exakte Übereinstimmungen existieren bei den Verbundgruppen

- Kinderbedarf

- Damenwäsche

- Selbst Schneidern.

Die weiteren Faktoren weisen vielfach nur noch eine sehr dominierende Abteilung auf und haben auch nur Eigenwerte knapp oberhalb von 1, so daß bei der Interpretation Probleme auftreten können bzw. keine komplexen Einkaufsbilder als Hintergrund entstehen.

$\mathrm{Zu}$ den dominierenden Abteilungen der restlichen Faktoren zählen die Abteilungen

- Uhren/Schmuck und Gardinen (Faktor 15)

- Restaurant (Faktor 16)

- Damenhüte (Faktor 17)

- Sommersport/Strand- und Badezubehör (Faktor 18) 
- Teppiche und Computer (Faktor 19).

Diese Abteilungen weisen in dieser Altersklasse offensichtlich so extreme Kaufintensitäten (sowohl über- als auch unterdurchschnittlich) auf, daß sie nicht einem der komplexeren Faktoren zuzuordnen sind. Dies erklärt sich durch die Spezifität der Bedürfnisse einer Alterklasse (z.B. haben die Damenhüte in dieser Altersgruppe eine Kaufintensität von $0,4)$.

Die Analyse der Daten der Altersgruppe von 36 bis 50 Jahren ergibt nur 16 Faktoren, wobei wiederum die ersten 14 Faktoren sehr plausible Kombinationen wiedergeben und Interpretationsprobleme erst bei den beiden letzten Faktoren auftreten.

Bei den ersten neun Faktoren der Analyse zeigte sich eine klare Analogie zwischen den Ergebnissen der Grundgesamtheit und dieser Altersgruppe. Diese Faktoren, die auch den höchsten Erklärungsanteil aufweisen, zeigen sich relativ stabil, auftretende Abweichungen sind plausibel. Auch in den weiteren Faktoren dieser Altersgruppe sind noch Ähnlichkeiten zur Grundgesamtheit zu erkennen. Doch sind auch zunehmend Abteilungen bei den faktorprägenden Bereichen, die bei der Grundgesamtheit in der Abteilungskombination nicht enthalten waren.

In der Altersgruppe der über 50jährigen ergeben sich 17 Faktoren, wobei sich wiederum Analogien zu Faktoren der Grundgesamtheit ergeben. Sowohl der Damen- als auch der Herrenmodebereich lösen sich jeweils in 2 Faktoren auf, wobei möglicherweise interpretatorisch eine altersgruppeninterne zusätzliche Alterstrennung nachzuvollziehen ist. Als Beispiel wird die Damenbekleidung detailliert dargestellt: 
Faktor 2 ist der erste Damenbekleidungsfaktor, der eher der jüngeren Dame über 50 zuzurechnen ist. Dazu gehören die Abteilungen Damenhosen, Röcke, Blusen, Jacken/Kostüme/ Kombinationsmoden, Damen-Wollwaren und Miederwaren als prägende Abteilungen mit Ladungswerten über 0,5. Als Randsortimente kommen noch Damen-Tageswäsche, Junge Mode mit Jeans- und Markenshops sowie Schuhe $(0,37$ - 0,39) hinzu. Der zweite Damenbekleidungsfaktor ist Faktor 6 , dessen bestimmende Abteilungen Damenhüte, Handschuhe und Modeaccessoires sind, was auf eher seriöse Mode hindeutet. Als starke Randsortimente kommen dabei noch Damen-Tageswäsche, Damen-Nachtwäsche und Hausschuhe $(0,39-0,43)$ hinzu. Darüber hinaus ist auch Faktor 12 als Damenbekleidungsfaktor $z u$ interpretieren. Dominierend sind bei diesem Faktor die Abteilungen Kleider und Damenmäntel sowie als Randsortimente Damenschuhe $(0,46)$, Röcke, Hausschuhe und Lederwaren. Insgesamt zeigt sich durch die Verteilung der Damenoberbekleidungssortimente auf 3 unterschiedliche Faktoren eine Polarisierung des Kaufverhaltens im Bereich der Altersgruppe über 50 Jahre.

Zudem wurde für den Bereich der Altersklassen die Arbeitshypothese $\mathbf{H}_{\text {NRII }}$ bestätigt. Die Kaufintensitäten waren in verschiedenen Klassen unterschiedlich ausgeprägt. Die Faktorenstrukturen sind jedoch weitgehend stabil.

Zusammenfassend ist festzustellen, daß die Analyse in den Altersgruppen zum einen die stabilität der überwiegenden Zahl der bei der Grundgesamtheit ermittelten Faktoren gezeigt hat. Zum anderen sind Abweichungen vom Grundmuster innerhalb der Altersgruppe plausibel $z u$ begründen. Dabei ist insbesondere auf die Aufspaltung von Damen- und Herrenbekleidungssegmenten $\mathrm{zu}$ verweisen, wie sie exemplarisch anhand der Damenkleidung in der Altersgruppe über 50 Jahre aufgezeigt wurde. Somit hat sich das Verfahren trotz der geringen Schwächen bei den Faktoren mit geringem Eigenwert in den Un- 
tergruppen bewährt und $z u$ aussagefähigen Ergebnissen geführt.

\subsection{Faktorenbildung in ausgewählten Einkommensgruppen}

Im Gegensatz zur Einteilung in Altersgruppen, die sich aufgrund $z u$ geringer Fallzahlen in den Randgruppen auf drei Gruppen beschränken mußte, wird die Grundgesamtheit auf vier Einkommensklassen verteilt. Innerhalb dieser Gruppen wird analog zum Vorgehen bei den Altersklassen eine Faktorenanalyse durchgeführt. Die Faktoren werden wiederum nach dem Kaiser-Kriterium begrenzt und Varimax-rotiert, bevor sie interpretiert werden.

Auch bei der Interpretation innerhalb der Einkommensklassen wird das Hauptaugenmerk auf die Entwicklung der Werte komplexer Bereiche und auf einen Vergleich der Ergebnisse einer Gruppe mit denen anderer Einkommensgruppen sowie der Grundgesamtheit gelegt. Dabei werden wiederum nicht alle Abteilungen einzeln aufgezählt und deren Ladungswerte genannt, um eine unnötig komplexe Darstellung zu vermeiden.

Die erste Einkommensgruppe ist die Gruppe mit bis $z u \quad 36.000$ DM Haushaltsnettoeinkommen pro Jahr. Bei der Analyse der Daten wurden insgesamt 18 Faktoren ermittelt. Auch hier sind wieder starke Ähnlichkeiten mit den Kombinationen der Grundgesamtheit zu finden. Es kommt wie in den Altersgruppen wiederum $z u$ einer spaltung eines sortimentes auf 2 Faktoren. Als Beispiel soll hier der Sportbereich dienen:

Der sport zerfällt in dieser Altersgruppe eindeutig in zwei Faktoren, von denen Faktor 9 den sommersport und Faktor 13 den wintersport repräsentiert. Zur Abteilung Sommersport/strand- und Badezubehör kommen die Abteilungen sportbekleidung und sportartikel sowie die Bekleidungssortimente 
Men's Fashion und Junge Mode mit Jeans- und Markenshops. Dabei weist die Abteilung Sportartikel mit 0,33 die schwächste Ladung auf, so daß der Sommerfaktor stark textilorientiert erscheint, wozu auch die zusätzlichen Bekleidungssortimente passen.

Faktor 13 ist eher sportartikelorientiert $(0,62)$ und umfaßt zusätzlich die Abteilungen wintersport $(0,78)$ und sportbekleidung $(0,43)$. Dazu tritt noch die Abteilung Hüte. Insgesamt weisen beide sportfaktoren relativ starke Ähnlichkeiten zu Faktor. 9 der Grundgesamtheit auf, wenn auch $z u$ beiden noch andere Sortimente hinzugenommen werden.

Zusammenfassend ist festzustellen, daß die für diese Einkommensgruppe ermittelten Faktoren vor allem bei den zentralen Faktoren (Faktoren mit hohem Eigenwert) auch in dieser Gruppe stabil sind. Darüber hinaus ist festzuhalten, daß einige Faktoren "zersplittern" und dann, z.T. ohne zusätzliche andere Abteilungen mit hohen Ladungswerten aufzuweisen, separat bestehen.

Bei der Analyse der Einkommengruppe mit einem jährlichen Haushaltsnettoeinkommen zwischen 36.000 und 50.000 DM ergeben sich insgesamt 16 Faktoren, die ebenfalls weitgehend bekannte Kombinationen von Abteilungen bilden. Als Beispiel dient hierbei die Gruppe Hobby und Freizeit:

Im Faktor 6 sind die Abteilungen des Hobby- und Freizeitbereiches enthalten, wobei nur dadurch Abweichungen zum Faktor 4 der Grundgesamtheit bestehen, als die Do-it-yourself-Abteilungen, die in Faktor 5 dieser Einkommensklasse mitbestimmend waren, hier nur noch als Randsortimente (Ladung $0,30$ bis 0,35$)$ in Erscheinung treten. Die Abteilungskombination an sich bleibt jedoch davon unberührt. 
Die Analyse der Einkommensgruppe von 50.000 - 80.000 DM ergab 17 Faktoren, die auch weitgehend die Kombinationen widerspiegeln, die bereits bei der Gesamtstichprobe ermittelt wurden. Allerdings bestehen verstärkt Verteilungen der sortimente auf zwei Faktoren, vor allem in den Bereichen

- Damenkleidung (Faktoren 3 und 7 sowie z.T. 17)

- Damenwäsche (Faktoren 7 und 16)

- Sport (Faktoren 10 und 15)

- Einrichtung (Faktoren 13 und 14).

Die letzte untersuchte Einkommensgruppe ist die Gruppe der Kartenkunden mit mehr als 80.000 DM Jahreshaushaltseinkommen. Bei der Analyse der Daten dieser Gruppe ergaben sich 19 Faktoren. Da diese Einkommensgruppe die geringste Fallzahl aller Untergruppen (sowohl Alters- als auch Einkommensklassen) aufweist, sind hier auch die am wenigsten ausgeprägten Ergebnisse bei Faktoren mit schwachen Eigenwerten $z u$ erwarten. So lassen sich für die Faktoren 12, 14, 15, 18 und 19 dieser Gruppe kaum Ähnlichkeiten mit Faktoren der Gesamtstichprobe ermitteln.

Von den Faktoren der Grundgesamtheit aus gesehen, findet sich jedoch sogar in dieser am schwächsten strukturierten Untergruppe eine Entsprechung. Die Faktoren

- Kinderbedarf

- Damenwäsche und

- Selbst Schneidern

werden nahezu exakt abgebildet. Nur der Faktor Shopping, der allerdings schon bei der Grundgesamtheit problematisch zu präzisieren war, wird nicht bzw. kaum aufgegriffen.

Insgesamt hat sich aber auch bei der Analyse innerhalb der Einkommensklasse die stabilität der bei der Grundgesamtheit 
ermittelten Faktoren gezeigt. Die größten Abweichungen entstanden wie erwartet insgesamt bei der Gruppe mit der geringsten Fallzahl, da dort die Prägnanz der Ergebnisse zwangsläufig schwächer war. Ebenso verhält es sich innerhalb der Gruppen bei den Faktoren mit den geringsten Eigenwerten. Diese weisen auch die am wenigsten prägnanten Bedarfsbilder auf.

Für die Einkommensklassen kann die Arbeitshypothese $\mathbf{H}_{\text {NKII }}$ als bestätigt angesehen werden. Die grundlegenden strukturen der Faktoren der Grundgesamtheit sind auch in den unterschiedlichen Einkommensgruppen vorhanden. Somit sind für das Marketing die Faktoren der Grundgesamtheit und die Kaufintensitäten in den Untergruppen von Bedeutung. Da die Faktorstrukturen in allen Untergruppen gleich sind, kann ein umfassendes Konzept z.B. im Rahmen eines Data-Base Marketing für die gesamte Gruppe der Kundenkartenbesitzer entwickelt werden. Über die Einbeziehung bestimmter Gruppen in spezielle werbeaktionen entscheidet dann die Kaufintensität einer Gruppe bzgl. des jeweiligen Produktangebotes.

Die Intensitäten geben z.B. auch Aufschluß über die optimale Zielgruppenausrichtung bestimmter Abteilungskombinationen. Diese Bereiche sollten jeweils auf die Untergruppe mit der höchsten Kaufintensität ausgerichtet werden. Problematisch ist dies nur bei Abteilungen, die auf verschiedene Faktoren laden. Dann kann es zu einem Konflikt bei der Zielgruppenausrichtung kommen. 
4. Zielgruppenbildung anhand von Verbundbeziehungen im Einzelhandel

\subsection{Der Nachfrageverbund als Ausgangspunkt der zielgruppen- bildung}

Mit Hilfe der Nachfrageverbundanalyse wurden komplexe Nachfragestrukturen aufgedeckt, die ein spezifisches Kaufverhalten widerspiegeln. Die dabei ermittelten Faktoren dienen nun als Ausgangsvariablen für eine Kundensegmentierung. Dabei genügen diese neu gebildeten Variablen allen sechs Anforderungen, die an Kriterien zur Marktsegmentierung gestellt werden: 22

1. Raufverhaltensrelevanz: Die abgeleiteten gemeinsamen Verhaltensmuster der kombinierten Abteilungskäufe wurden gezielt so ausgewählt, daß sie gleichzeitig kaufauslösende Motive umfassen, indem sie komplexe Bedarfsmuster aufzeigen.

2. Aussagefähigkeit für den Einsatz der Marketinginstrumente: Die Basis der neu gebildeten Kriterien sind die tatsächlichen Käufe der untersuchten Kunden. Daher ist die Möglichkeit für einen gezielten Einsatz der Marketinginstrumente ebenso evident wie auch inhaltlich einfach zu gestalten.

3. Wenn die $\mathrm{zu}$ erhebenden Ausgangsdaten (Kaufinformationen) vorliegen, ist über die direkte zuordnung von Kundeninformationen über die Kundenkarte die zugänglichkeit eindeutig gewährleistet. Darüber hinausgehende Anwendungsmöglichkeiten auf Konsumentengruppen, über die weniger oder andere Informationen vorliegen, müssen erst anhand

22 Vgl. Freter, H., Marktsegmentierung, a.a.O., S. 43 und die dort angegebene Literatur. 
der Homogenität der erzielten Kundengruppen in bezug auf leicht zugängliche Informationen (Alter, Geschlecht, Einkommen etc.) überprüft werden.

4. Die Entstehung der Kriterien sowie die Herkunft der Daten zeigen automatisch die operationalisierung (in diesem Falle MeBbarkeit durch Kaufintensitäten und deren Ladung auf gemeinsame Faktoren) und auch die zugänglichkeit durch die Methoden der Marktforschung (einfache Entnahme der Daten aus dem Warenwirtschaftssystem eines Handelsunternehmens). Die Daten wären ferner, wenn auch in minderer Quantität und evtl. Qualität, durch Beobachtung und Befragung zu ermitteln.

5. Die ermittelten Faktoren sind als zeitraumbezogenes Konstrukt grundsätzlich durch eine gewisse zeitliche stabilität gekennzeichnet. Darüber hinaus wurde zum anderen die detaillierte Analyse in den unterschiedlichen Untergruppen, insbesondere den verschiedenen Altersgruppen, durchgeführt, um diese stabilität zu überprüfen.

6. Die Wirtschaftlichkeit der Datenerhebung ist gegeben. Die Informationen stehen durch die EDV-abhängige Nutzung der Kundenkarte automatisch ohne Mehraufwand zur Verfügung. Eine wirtschaftliche Eignung der gebildeten segmente ist vor dem Hintergrund der hohen Kaufverhaltensorientierung der Kriterien anzunehmen.

Abschließend ist noch festzustellen, welcher Kriteriengruppe die hier verwendeten Faktoren zuzuordnen sind. Eindeutig sind sie weder den Reaktionskoeffizienten noch den sogenannten Ersatzkriterien, wie sozioökonomischen, psychographischen oder beobachtbaren Kaufverhaltensgrößen zuzuordnen. ${ }^{23}$

23 Vgl. zu den Kriteriengruppen Freter, H., Marktsegmentierung, a.a.o., S. $44 \mathrm{f}$. 
Sie beruhen auf beobachtbaren Kaufverhaltensgrößen durch die Dokumentation des getätigten Kaufs. Letztendlich sind sie durch die bedarfs- und motivorientierte Interpretation der vorliegenden Kaufintensitäten eher den psychographischen Marktsegmentierungskriterien zuzurechnen.

\subsection{Kundensegmentierung auf der Basis von Nachfrageverbund- beziehungen}

Bei der Clusteranalyse auf Basis der Faktoren der Nachfrageverbundanalyse ergeben sich 5 cluster. Als Methode wurde die Ward-Methode benutzt und als Distanzmaß die quadrierte euklidsche Distanz. Aufgrund einer Diskriminanzanalyse ist festzustellen, daß die vorliegende Gruppenbildung als trennscharf zu bezeichnen ist.

Zur Interpretation der cluster werden zum einen die Faktoren herangezogen, die auf Basis der Grundgesamtheit ermittelt wurden und die sich als stabil gezeigt haben. Zum anderen werden drei soziodemographische Parameter bei der Interpretation untersucht um festzustellen, inwiefern die gebildeten Kundengruppen, die sich durch gleiche Kaufkombinationen auszeichnen, auch gemeinsame soziodemographische Merkmale aufweisen. Die benutzten Kriterien sind:

- Das Kundenalter in den 5 ursprünglichen Ausprägungen (1825 Jahre, 26-35 Jahre, 36-50 Jahre, 51-65 Jahre und mehr als 65 Jahre),

- das Geschlecht des Kundenkartenbesitzers,

- das Einkommen in den 4 bereits bei der Faktorenanalyse benutzten Gruppen (bis 36.000 DM, 36.001 - 50.000 DM, 50.001 - 80.000 DM, mehr als 80.000 DM).

Die Interpretation dieser Größen erfolgt im Vergleich zur jeweiligen Verteilung in der Grundgesamtheit, die als durch- 
schnittlich angesehen wird. Z.B. wird in cluster 4 die Altersklasse von 51-65 Jahren als um $12 \%$ größer als im Durchschnitt beschrieben. Dies ist die absolute Abweichung (Durchschnitt ca. $26 \%$, Cluster $4 \mathrm{ca} .38 \%$ ) und bedeutet eine relative Erhöhung um fast $50 \%$, wenn die durchschnittliche Prozentzahl und die Prozentzahl der Gruppe als Quotient berechnet werden.

Damit bei Änderungen in Gruppen mit kleinen Prozentzahlen bei nur kleinen absoluten Abweichungen durch hohe relative Abweichungen kein falsches Bild entsteht (z.B. 0,2 \% absolute Änderung bei $2 \%$ Basis entsprechen $10 \%$ relativer Änderung, obwohl dies eventuell nur sehr wenige Fälle sind), wird die absolute Darstellung gewählt.

Die Diskriminanzanalyse ergab eine zuordnungswahrscheinlichkeit von $81,2 \%$ der Fälle. Daraus ist abzuleiten, das die clusterzuordnung der einzelnen Fälle stabil ist. Dafür sprechen auch die wilks Lambda Werte von $0,11,0,21,0,39$ und 0,59 . Insgesamt ergibt sich daher eine tragfähige Gruppenbildung.

Bei einer Betrachtung der Fallzahlen, die jedem cluster zugeordnet sind, zeigt sich direkt, daß es ein cluster gibt, das mit 2.285 Fällen insgesamt 55,1 \% aller Kunden enthält. Dieses Cluster spaltet sich auch bei einer Erhöhung der clusterzahl zunächst nicht weiter auf. Stattdessen werden andere cluster weiter unterteilt, so daß deren Fallzahlen weiter sinken. 24 Daher wurde für diese Interpretation die 5cluster-Lösung zugrunde gelegt.

24 Dies bestätigt eine Untersuchung der Fehlerquadratsumme nach dem Ellbow-Kriterium, die auf eine 5 cluster-Lösung deutet. 


\section{cluster 1:}

\section{schlechter Verdienende über 36 Jahre $(642$ Fälle $=16,0 \%)$}

$75 \%$ der in diesem cluster zusammengefaßten Kunden sind Frauen. Deutlich erkennbar ist, daß zudem tendenziell Kunden der oberen Altersklassen vertreten sind. So sind nur 9,3 \% der Personen in der Altersgruppe von 26-35 Jahre. Dafür liegt die Altersgruppe von 36-50 Jahren ca. $3 \%$ und die von 51-65 Jahren sogar $5 \%$ über dem Durchschnitt. Auch die Klasse über 65 Jahren ist noch $1 \%$ stärker als im Durchschnitt.

Bei den Einkommensklassen hingegen liegt die oberste Gruppe, die auch die kleinste ist, um $5 \%$ unter dem wert der Grundgesamtheit. Dafür sind die untersten beiden Gruppen jeweils um ca. 3 \% erhöht.

In bezug auf die Kauffaktoren ist festzustellen, daß Damenwäsche und Damenkleidung die höchsten Werte aufweisen $(0,97$ und 0,83). Eine weitere starke Gruppe bilden die Faktoren Einrichtung und Haushaltsausstattung $(0,36$ und 0,35). Auch der "Schlechtes Wetter"-Faktor weist mit 0,21 noch einen prägnanten Wert auf. Alle übrigen Faktoren liegen z.T. positiv, aber $z . T$. auch negativ relativ nahe am Durchschnittswert 0 , so daß sie keine prägnanten Interpretationen zulassen.

\section{Cluster 2:}

Gutsituierte Konsumenten mittleren Alters (687 Fälle=16,6 \%) $63,3 \%$ der Kunden, die diesem cluster zugeordnet wurden, sind Männer. Die Gruppe ist u.a. dadurch gekennzeichnet, daß eine deutlich vergrößerte Gruppe der mittleren Altersklasse von 36-50 Jahren festzustellen ist. Die 5 Prozent, um die diese Gruppe vergrößert ist, fehlen bei den älteren Kundengruppen von 51-65 Jahren mit ca. $1,5 \%$ und bei den mehr als 
$65 j a ̈ h r i g e n ~ s o g a r ~ m i t ~ 3 \%$. Die jüngeren Gruppen liegen in etwa im Durchschnitt.

Die Einkommensverteilung zeigt eine deutlich stärkere Ausprägung der oberen Gehaltsklassen um $2 \%$ bei 50.000 - 80.000 DM und sogar $3 \%$ bei über 80.000 DM Einkommen. Analog fehlen bei den beiden unteren Gehaltsgruppen jeweils ca. 2,5\% gegenüber den Werten der Grundgesamtheit.

Deutlich bestimmt wird die Kaufseite dieser Gruppe vom Bereich der Herrenkleidung $(1,1)$, was auch mit dem hohen Anteil männlicher Kundenkartenbesitzer in dieser Gruppe korrespondiert. Eine zweite bestimmende Größe ist die Verbundgruppe sport $(0,7)$. Aber auch der Bereich der Accessoires für Damen $(0,5)$ stellt in dieser Gruppe einen Kauffaktor dar. Die Abteilungen dieses Faktors (Hüte, Handschuhe etc.) könnten im Kontext dieser Gruppe als Geschenkbereich dienen. Unterdurchschnittliche Werte, die relativ ausgeprägt sind, weisen die Faktoren Kinderbedarf und Damenkleidung mit je ca. -0,2 auf, so daß man von einer vielzahl alleinstehender junger Männer in diesem Cluster ausgehen kann. Die restlichen Faktoren schwanken wiederum in einem relativ kleinen Intervall um den Durchschnittswert.

\section{cluster 3:}

\section{Durchschnittskunden $(2.285$ Fälle $=55,1 \%)$}

Dieses cluster umfaßt die meisten Kunden und aus diesem Grund sind Abweichungen vom Durchschnitt weniger zu erwarten als bei den kleineren clustern. Markante Abweichungen sind daher um so stärker zu berücksichtigen. Sollte sich eine derartig große Gruppe auch bei späteren Analysen bestätigen, so ist davon auszugehen, daß die in diesem cluster vertretenen Kunden jeweils ohne spezielle Kaufabsicht die Geschäftsstätte aufsuchen bzw. ihren Bedarf in bestimmten segmenten nicht schwerpunktmäßig dort decken. Für diese Argumentation spricht, daß die Kunden dieses clusters mit einem durch- 
schnittlichen Kaufbetrag von nur ca. 343 DM pro Jahr relativ stark unter dem Durchschnitt von 1.831 DM liegen. Dies kennzeichnet den typischen Warenhauskunden, der keinen spezifischen Bedarf deckt und somit für eine marketingbezogene Erfassung kaum geeignet ist.

In soziodemographischer Hinsicht ist festzustellen, daß die mittlere Altersklasse $4 \%$ kleiner ist als im Durchschnitt. Dafür sind alle anderen Altersklassen um ca. $1 \%$ größer. Im Hinblick auf die Annahme, daß dieses cluster die eher "unspezifischen" Kunden enthält, kann diese Verteilung als Argumentationshilfe herangezogen werden. Bei den jüngeren Altersgruppen ist das Kaufverhalten häufig noch nicht so ausgeprägt habitualisiert wie im mittleren Alter. Im höheren Alter dagegen ist zwar der Grundbedarf festgelegt, darüber hinaus gehende Kaufbedürfnisse sind zumeist weniger ausgeprägt.

Dieses Cluster enthält mit $57,6 \%$ überwiegend männliche Kartenbesitzer. Die Einkommensverteilung liegt fast genau in Höhe der Durchschnittswerte, so daß aus diesem Aspekt kaum Erkenntnisse gewonnen werden können.

Auch die Werteverteilung bei den Nachfrageverbundfaktoren unterstützt die Annahmen über die Mitglieder dieses Clusters. So gibt es keinen Faktor, der eine ausgeprägte Mittelwertabweichung aufweist. Der höchste positive wert ist mit 0,04 beim Hobby und Freizeitfaktor festzustellen. Es gibt allerdings einige Bereiche, die mit einer relativ schwachen Ausprägung von ca. -0,2 nicht gekauft werden. Dies sind Kinderbedarf, Damenwäsche, Herrenkleidung und bedingt auch Damenkleidung und sport. Das bedeutet vor allem eine Ablehnung der textilen sortimente. Es werden von dieser Gruppe hauptsächlich Waren erworben, für die keine Fachgeschäfte existieren (z.B. viele Bereiche der Hartwaren). 


\section{cluster 4:}

Ältere gutsituierte Konsumenten (96 Fälle $=2,3 \%$ )

Dieses am geringsten besetzte cluster weist eine sehr stark ausgeprägte soziodemographische struktur auf. $60 \%$ der Clustermitglieder sind weiblichen Geschlechts. Sie entstammen den höheren Altersklassen. So liegt die Gruppe von 51-65 Jahren um $12 \%$ und die Gruppe über 65 Jahren um $2 \%$ über dem Durchschnitt.

Die Einkommensstruktur weist ähnlich krasse Ausprägungen auf. So liegt die Gehaltsgruppe von 50.000 - 80.000 DM um 7 $\%$ und die über 80.000 DM um $1 \%$ über dem Wert der Grundgesamtheit. Dafür ist die Gruppe unter 36.000 DM mit nur noch $16,7 \%$ um $6 \%$ kleiner als bei der Gesamtstichprobe.

Die Fallzahl von 96 Kunden in diesem cluster weist bereits darauf hin, daß diese Gruppe ein extremes und spezifisches Kaufmuster aufweist. Geprägt wird dieses cluster durch einen extrem hohen wert $(5,55)$ des Faktors selbst Schneidern. Dieser Wert stellt mit Abstand die stärkste Ausprägung aller Faktoren in allen clustern dar. Aus dem Kaufverhalten der Personen dieses spezialclusters resultiert auch die extreme Stabilität des Faktors bei der Untergruppenanalyse. AuBerdem weisen noch die Faktoren Einrichtung $(0,4)$ und Grundbedarf $(0,32)$ prägnante werte auf. Aber auch im Bereich des unterdurchschnittlichen Kaufs sind relativ deutliche Ausprägungen vorhanden. Dazu zählen Damen-Accessoires $(-0,33)$, Damenkleidung $(-0,31)$ und "älterer Herr" $(-0,28)$.

\section{Cluster 5: Gutsituierte jüngere Familie (416 Fälle $=10,0 \%$ )}

Dieses cluster enthält zu gleichen Anteilen männliche und weibliche Kundenkartenbesitzer. Bestimmend im Altersbereich sind die Gruppen 26-35 Jahre $+12 \%$ und 36-50 Jahre $+13 \%$ im Vergleich $z u$ den Werten der Grundgesamtheit. Zusammen machen beide Gruppen $88,9 \%$ der Gruppenmitglieder aus, wohingegen die Altersgruppen unter 25 und über 65 Jahre zusammen nur 
$2,7 \%$ ausmachen, so daß sie vernachlässigt werden können. Insgesamt ist diese Gruppe durch das mit Abstand geringste Durchschnittsalter gekennzeichnet.

Im Einkommensbereich ist eher eine Tendenz zu höheren Einkommensgruppen feststellbar. Die unteren beiden Einkommensklassen liegen $3 \%$ (bis 36.000 DM) bzw. $2 \%(36.001-50.000$ DM) unter dem Durchschnitt, wohingegen die Gehaltsklasse über 80.000 DM mit $4 \%$ über dem Wert der Grundgesamtheit extrem hoch ist.

Die Analyse der Kauffaktoren zeigt das Bild einer jungen Familie, die ihre Ausgaben stark auf den Kinderbedarf konzentriert und darüber hinaus eher durchschnittlich einkauft. Dies zeigt ein Wert von 2,5 für den Bereich Kinderbedarf, der sich auch bei der Analyse als sehr stabiler Faktor erwies. Der nächststärkere Kauffaktor ist sport $(0,15)$, der ebenfalls eine klare überdurchschnittliche Ausprägung zeigt. Dies war aber aufgrund der überwiegend jüngeren clustermitglieder trotz der Konzentration auf den Kinderbedarf zu erwarten. Die übrigen werte orientieren sich weitgehend am Durchschnitt.

Insgesamt erbrachte die clusteranalyse Gruppen mit konsistenten Kaufmustern, die - je nach struktur des Segmentes werblich bearbeitet werden können. Einzig die undifferenzierte Gruppe der Durchschnittskunden des Clusters 3 stellt sich bei der Ableitung von Kaufmustern problematisch dar und sollte in späteren Untersuchungen überprüft werden. Diese Durchschnittskunden stellen ein warenhaustypisches problem dar, das vor allem durch eine mangelnde Zielgruppenausrichtung bedingt ist. Bei anderen Vertriebsformen wird daher eine solche Gruppe zumindest so stark ausgeprägt nicht auftreten. Die Wirtschaftlichkeit von Werbemaßnahmen in den ermittelten segmenten hängt stark von der Größe der jeweiligen Gruppe und der Zielsetzung des Handelsunternehmens ab. 


\subsection{Die Gruppe der inaktiven Kartenbesitzer}

Im Zusammenhang mit einer Analyse unterschiedlicher Zielgruppen auf der Basis von Verbundinformationen, die sich durch die Auswertung von Kaufdaten von Kundenkartenbesitzern ergeben, ist die Gruppe der inaktiven Kartenbesitzer in die Betrachtung einzubeziehen. ${ }^{25}$ Dabei ist die Untersuchung der demographischen struktur dieser Gruppe von besonderem Interesse. Sollten sich definitive demographische schwerpunkte feststellen lassen, so könnten daran interessante Hypothesen über die Akzeptanz bestimmter Sortimentsgruppen abgeleitet werden. Diese hypothetischen wirkungen können dann wiederum starke Einflüsse auf die Zusammensetzung des Kauf- und Nachfrageverbundes haben.

Darüber hinaus ist es für das Handelsunternehmen möglich, durch spezifische Angebotsgestaltungen die inaktiven Karten zu aktivieren. Dazu kann der spezifische Verbund einer bestimmten demographischen Gruppe herangezogen werden, deren demographische charakteristika sich mit einer schwerpunktgruppe der inaktiven Kunden decken. Ein auf dieser Basis gezielt aufgebautes Angebot bietet wesentlich größere Erfolgsaussichten als ein diffuses Angebot für eine breite zielgruppe. Zudem ist es dadurch möglich, den streuverlust der Werbung zu reduzieren und somit Kosten zu sparen.

Insgesamt besteht diese Gruppe aus 1.279 Kunden, die im gesamten Geschäftsjahr die Kundenkarte nicht genutzt haben. Wenn die Verteilung auf verschiedene Einkommensklassen näher betrachtet wird, so fällt auf, daß überwiegend höhere Einkommensklassen (gemessen an ihrem Anteil am Gesamtbestand) $z u$ den Inaktiven zählen. Dies erscheint zunächst überraschend, da aufgrund der Entwicklung der Kundenkarten aus dem

25 Auch Nicht-Käufer bilden ein segment, das zu einer umfassenden Marktsegmentierung gehört. Vgl. Freter, H., Marktsegmentierung, a.a.0., S. 21 . 
Kreditkartenwesen diese Kundenschicht an den Gebrauch einer solchen Karte gewohnt sein müßte.

Darüber hinaus kommt hier jedoch ein anderer Faktor zur wirkung. So wird die Karte häufig gerade bei niedrigeren Einkommen als Möglichkeit angesehen, kostengünstig (zinslos) eine Kreditaufnahme für kürzere Zeiten zur überbrückung eines kurzfristigen Defizites durchzuführen. ${ }^{26}$ Dazu wird wahrscheinlich das Argument des $3 \%$ Rabattes bei Kunden mit niedrigerem Einkommen wirkungsvoller aufgenommen als in den Kundengruppen mit höherem Einkommen.

Bei einer Betrachtung der Verteilung der inaktiven Runden bzgl. der Altersgruppen ist eine Polarisierung festzustellen. So sind verhältnismäßig wenige Inaktive aus den mittleren Altersbereichen $z u$ verzeichnen (gemessen an ihrem Anteil am Gesamtbestand). Überwiegend entstammen die inaktiven Kunden den ganz jungen und ganz alten Kundengruppen.

Bei den "Alten" könnten Gründe der Einkaufsbequemlichkeit vorliegen, die verhindern, daß ware aus einem Warenhaus in Citylage beschafft wird. In diesem Falle könnte ein Heimlieferservice für bestimmte Angebotspakete, die aus dem Nachfrageverbund dieser Gruppe abgeleitet sind, zu guten Ergebnissen führen. Ein zweiter Grund für die Inaktivität in dieser Gruppe könnte die mangelnde Ausrichtung des Einkaufserlebnisses 27 auf diese spezifische Gruppe sein.

26 Dies fand das untersuchte Handelsunternehmen anläßlich einer Befragung von 1.000 Kartenbesitzern im Jahre 1989 heraus.

27 Zum Begriff Einkaufserlebnis vgl. z.B. Heinemann, G., Betriebstypenprofilierung und Erlebnishandel, a.a.o.; Ahlert, D., Schröder, H., Erlebnisorientierung im stationären Einzelhandel, a.a.O., S. $221 \mathrm{ff}$. 
Das Einkaufserlebnis ist auf der anderen seite kaum ein primärer Grund für die Inaktivität der jungen Altersgruppe. Hier ist eher $z u$ vermuten, daß das sortiment den besonders intensiven Anforderungen dieser Altersgruppe an originalität, Aktualität und Mode nicht gerecht wird. Ob diesem Trend allerdings durch ein Handelsunternehmen, das als Vollsortimenter auftritt, entgegengetreten werden kann und soll, ist von übergeordneten Entscheidungen abhängig, da eine solche spezialisierung eine einschneidende Entwicklung ist.

\section{Implikationen des Kauf- und Nachfrageverbundes für das Management im Einzelhandel}

\subsection{Rritische würdigung der Leistungsfähigkeit der Verbund- analyseverfahren}

Die Aussagefähigkeit der Kauf- und Nachfrageverbundanalysen für das Handelsmarketing ist insbesondere vom zugrundeliegenden Analyseinstrumentarium sowie der Art der Datenbasis abhängig. Deshalb ist kritisch zu hinterfragen, inwieweit das vorgestelite Instrumentarium auf der Grundlage von Kundenkarteninformationen im Vergleich $z u$ den bekannten und in der Literatur hinlänglich diskutierten Verfahren detailliertere und entscheidungsrelevantere Informationen für das Handelsmarketing liefern kann.

Im Rahmen der Kaufverbundanalyse wird die Datenanalysemethodik der bekannten Ansätze nicht verändert. Daher können in diesem Bereich Vorteile für das Handelsmarketing nur durch die Qualität der Datenbasis erzielt werden. Bislang werden drei Verfahren zur Erhebung von Verbunddaten für die Kaufverbundanalyse eingesetzt:

- die Befragung oder

- die Beobachtung von Konsumenten, 
- Scannerkassen bzw. die Auswertung von Kassenbelegen.

Alle drei Möglichkeiten weisen jedoch Schwächen auf. Die Befragung unterliegt z.B. ergebnisverzerrenden Intervieweffekten, und die Beobachtung ist sowohl sehr aufwendig als auch in ihrer Durchführung problematisch. 28 Der Einsatz von Scannerkassen bzw. die Auswertung von Kassenbelegen stellt im Vergleich dazu bereits eine qualitative Verbesserung dar. Die so ermittelten Daten sind weitgehend objektiv. Die Nutzung von Scannerkassen ermöglicht darüber hinaus direkt eine DV-mäßige Erfassung der Daten. Dies führt zur Aufhebung von quantitativen Schranken bei der Datenerhebung. Zudem sind ständig aktuelle Daten verfügbar.

Die Datenerfassung über Kundenkarten bietet über die Nutzung von Scannerkassen hinausgehende Vorteile. Diese entstehen durch die zuordnung von Personendaten zu den reinen Kaufdaten. Dadurch eröffnet sich die Möglichkeit, bestimmte Personengruppen in bezug auf ihre Verbundstrukturen zu untersuchen. Ebenso sind vergleichende Analysen zwischen den Verbundstrukturen verschiedener Personengruppen möglich. Darüber hinaus können Personen oder Personengruppen zu unterschiedlichen zeitpunkten identifiziert werden, so daß auch vergleichende Analysen im Zeitablauf realisierbar sind. Damit ist es möglich, Veränderungen im Verbundkaufverhalten festzustellen.

Die Identifizierbarkeit einzelner Kunden $z u$ verschiedenen Kaufzeitpunkten schafft darüber hinaus erstmals die Möglichkeit zur unternehmensspezifischen Nachfrageverbundanalyse. Als innovatives Konstrukt einer zeitraumbezogenen Verbund-

28 Auf eine detaillierte Diskussion grundlegender Schwächen von Befragungen und Beobachtungen wird an dieser stelle verzichtet. $\mathrm{Zu}$ Beobachtung und Befragung als Methoden der Datengewinnung vgl. z.B. Meffert, H., Marktforschung, a.a.0., s. 33 ff. 
größe ist es mit dem Konstrukt des Kaufverbundes kaum direkt vergleichbar. Im Vergleich zum Kaufverbund ist der Nachfrageverbund weniger situativ beeinflußt, da die Käufe verschiedener Kaufzeitpunkte zusammengefaßt werden. Daraus resultiert eine Annäherung an den Bedarfsverbund. 29 Darüber hinaus sind die Auswirkungen der angebotsbezogenen Einflußfaktoren nicht so stark ausgeprägt wie beim Kaufverbund. 30

Insgesamt ist somit davon auszugehen, daß Kundenkarteninformationen eine wesentliche Erweiterung des Spektrums möglicher Verbundanalysen eröffnen. Damit können Kundenkartendaten und darauf aufbauende Verbundanalysen wesentliche $\mathrm{zu}$ satzinformationen für das Handelsmarketing liefern. Dies hat auch die empirische Ermittlung des Kauf- und Nachfrageverbundes gezeigt. Beide Verfahren sind zur Abbildung plausibler Kaufstrukturen der Nachfrager geeignet.

Dabei ist zu berücksichtigen, daß im Rahmen dieser Untersuchung sowohl der Kauf-als auch der Nachfrageverbund auf Abteilungsebene eines Handelsunternehmens analysiert wurden. Daraus ist jedoch keine grundsätzliche Beschränkung der vorgestellten Verfahren auf Verbundbeziehungen zwischen Abteilungen abzuleiten. Die Vorgehensweise ist direkt auf andere Datenaggregationsniveaus übertragbar.

Der die Untersuchungsobjekte betreffende Aspekt der Verbundanalyse ( $z$. B. Artikel, Abteilung etc.) wird im folgenden als objektebene bezeichnet. Insgesamt können unabhängig von der objektebene z.B. für Einzelartikel oder Artikelgruppen mit der gleichen Aussagequalität wie für Abteilungen Kaufund Nachfrageverbundstrukturen errechnet werden.

Vgl. Kapitel B.1.1.

Vgl. Kapitel B.2.1.
} 
Allerdings ergeben sich bei unterschiedlichen objektaggregationen auch Beschränkungen. Dies betrifft vor allem die Handhabbarkeit der für die Analysen notwendigen Daten. Dabei steht die Komplexität der Datenmatrix und die Interpretierbarkeit der Ergebnisse im Vordergrund. Oberhalb einer bestimmten Anzahl unterschiedlicher Untersuchungsobjekte, zwischen denen Verbundbeziehungen aufgezeigt werden, ist die Analyse für eine handhabbare Interpretation aufgrund der exponentiell steigenden Zahl möglicher Verbundbeziehungen zu komplex. Wird bei einer Kauf- oder Nachfrageverbundanalyse daher ein besonders feines Aggregationsniveau auf der objektebene benötigt (z.B. Artikelniveau), so können nicht mehr alle Untersuchungsobjekte abgebildet werden. Deshalb ist für derartige Analysen eine Beschränkung der Kauf- und Nachfrageverbundanalyse auf einen Ausschnitt der Untersuchungsobjekte, z.B. die Artikel einer Artikelgruppe, erforderlich. Somit können mit steigender Verfeinerung des Aggregationsniveaus die Verbundbeziehungen aller Untersuchungsobjekte weniger umfassend abgebildet werden.

Die Wahl des Aggregationsniveaus auf der Ebene der Untersuchungspersonen ist ebenfalls $\mathrm{zu}$ hinterfragen. Dabei steht die Frage des Vorgehens auf der Individual- oder Gruppenebene im Vordergrund. In diesem Zusammenhang ist jedoch kaum eine reale Wahlmöglichkeit vorhanden. Die Verfahren der Kauf- und Nachfrageverbundanalyse setzen bereits die Rahmenbedingungen für diese Aggregationsproblematik.

Bei der Ermittlung des Kaufverbundes muß die Individual- und auch die Personengruppenebene bereits in einem sehr frühen Schritt aufgegeben werden. Nach der Übertragung der Kaufpare in die Datenmatrix für die clusteranalyse ist keine Möglichkeit zur Identifikation der Kaufverbundstrukturen eines Individuums mehr gegeben. Die Analyse der Kaufverbundbeziehungen einer bestimmten Gruppe von Personen kann erfolgen, wenn bereits vor dieser stufe ein Filter zur selektion 
der Untersuchungspersonen eingeführt wird. Dieser Filter kann z.B. aus einem oder der Kombination verschiedener soziodemographischer Merkmale der Untersuchungspersonen bestehen, die für eine vorgeschaltete clusteranalyse zur segmentierung eingesetzt werden.

Die Nachfrageverbundanalyse erweist sich in bezug auf die Aggregation der Personenebene als wesentlich flexibler. Da bei der Nachfrageverbundanalyse zunächst alle Käufe eines Kunden zusammengefaßt werden, kann auf alle, einem Konsumenten zugehörigen Personendaten bis zu den letzten Analyseschritten zurückgegriffen werden. Somit werden in der empirischen Analyse des Nachfrageverbundes die jeweiligen Kaufstrukturen von Individuen oder Personengruppen untersucht. Daraus ergibt sich zum Beispiel die Möglichkeit der vergleichenden Betrachtung von Alters- und Einkommensgruppen in bezug auf ihre Nachfrageverbundstruktur. 31

Die Bezugsebene "Individuum" ist darüber hinaus eine notwendige Voraussetzung für die zielgruppenbildung auf der Basis von Nachfrageverbundstrukturen. ${ }^{32}$ ohne eine Identifizierung der jedem Individuum zugeordneten Verbundwerte und soziodemographischen Daten ist weder eine Gruppenbildung noch eine fundierte clusterbeschreibung möglich.

Für unterschiedliche Entscheidungsprobleme des Einzelhandels werden verschiedene Aggregationsniveaus benötigt, die sowohl auf der objekt- als auch der Personenebene zu definieren sind. Da die vorliegende Untersuchung die Verbundbeziehungen zwischen Abteilungen analysiert, blieben bislang Entscheidungsprobleme, die andere Aggregationsniveaus benötigen, vernachlässigt. Abbildung 25 gibt einen Überblick über solche Entscheidungsprobleme des Handels, bei denen die Ver-

Vgl. Kapitel C.3.2.

Vgl. Kapitel C.4. 
bundforschung zur Entscheidungsfindung beiträgt, sowie die dazugehörenden Aggregationsniveaus der objekt- und Personenebene.

\begin{tabular}{|c|c|c|c|}
\hline \multirow{2}{*}{ ENTSCHEIDUNGSBEREICH } & \multicolumn{2}{|c|}{ AGGREGATIONSEBENE } & \multirow{2}{*}{$\begin{array}{l}\text { NOTWENDIGE } \\
\text { ZUSATZ- } \\
\text { INFORMATION }\end{array}$} \\
\hline & OBJEKT & PERSON & \\
\hline NEUPRODUKTEINFÜHRUNG & ARTIKEL & GESAMT & UMSÄTZE \\
\hline PREISSETZUNG & ARTIKEL & GESAMT & $\begin{array}{l}\text { UMSÄTZE/ } \\
\text { DB-STRUKTUR }\end{array}$ \\
\hline PLAZIERUNG & $\begin{array}{l}\text { ARTIKEL/ } \\
\text { ABTEILUNG }\end{array}$ & GESAMT & --- \\
\hline SORTIMENTSBEREINIGUNG & $\begin{array}{l}\text { ARTIKEL/ } \\
\text { ABTEILUNG }\end{array}$ & GESAMT & UMSÄTZE \\
\hline ZIELGRUPPENBILDUNG & $\begin{array}{l}\text { ARTIKEL/ } \\
\text { ABTEILUNG }\end{array}$ & INDIVIDUAL & $\begin{array}{l}\text { PERSONEN- } \\
\text { DATEN }\end{array}$ \\
\hline WERBEWIRKUNGSKONTROLLE & $\begin{array}{l}\text { ARTIKEL/ } \\
\text { ABTEILUNG }\end{array}$ & $\begin{array}{l}\text { INDIVIDUALI } \\
\text { GRUPPEI } \\
\text { GESAMT } \\
\end{array}$ & $\begin{array}{l}\text { PERSONEN- } \\
\text { DATEN }\end{array}$ \\
\hline KOMMUNIKATION (DATA-BASE) & $\begin{array}{l}\text { ARTIKEL/ } \\
\text { ABTEILUNG }\end{array}$ & INDIVIDUAL & $\begin{array}{l}\text { PERSONEN- } \\
\text { DATEN }\end{array}$ \\
\hline GESCHÄFTSBEREICHPLANUNG & ABTEILUNG & GESAMT & - \\
\hline
\end{tabular}

Abb. 25: Entscheidungsbereiche des Handels mit Bezug zur Verbundmessung

Dabei zeigt sich, daß z.B. die Preispolitik als verbundbezogener Entscheidungsbereich nur auf der Artikelebene gelöst werden kann. Voraussetzung dafür ist, daß der Einzelartikel in seiner wirtschaftlichkeit nicht nur isoliert betrachtet wird, 33 sondern auch seine Kaufverbundbeziehungen $\mathrm{zu}$ anderen Artikeln sowie deren wirtschaftlichkeit einbezogen werden, so daß jeder Artikel eine komplexe Bewertung erfährt. Hier- 
bei führt die Einbeziehung der Deckungsbeiträge zu realistischen wirtschaftlichkeitswerten. Dieses Vorgehen führt wenngleich sehr aufwenig - zu einer sehr genauen Erfassung der Wirtschaftlichkeit eines Artikels.

Ein weiteres Beispiel auf der Artikelebene stellt die überprüfung der Neuaufnahme eines Produkts in das sortiment dar. Hier wurde bisher nur der reine Abverkauf des Neuproduktes an seinen stückzahlen gemessen. Für eine umfassende Bewertung bietet sich aber eine Analyse der Kaufverbundstruktur an um festzustelien, ob das Neuprodukt eher komplementär oder substitutiv $z u$ anderen Artikeln ist. Auch die stärke seiner Anbindung an andere Produkte ist feststellbar. Darüber hinaus kann durch Beobachtung einer Gruppe mit konstanter Besetzung $z u$ verschiedenen Zeitpunkten festgestellt werden, ob das Produkt durch Wiederholungskäufe zu einem festen Bestand der Käufe bestimmter Personen wird. Als Beispiel soll die Einführung eines ökologieorientierten produktes dienen. Mit Hilfe der Verbundanalyse ist einerseits feststellbar, wie stark das produkt mit anderen in einem Kaufverbund steht. Andererseits gibt eine Untersuchung von Kaufund Nachfrageverbund Aufschluß darüber, ob ein verbund zu anderen ökologieorientierten produkten besteht. Ist ein starker Zusammenhang zwischen den Käufen ökologieorientierter Produkte feststellbar, so kann über die Prüfung des Neuproduktes hinaus ein allgemeiner Trend abgeleitet werden, der Hinweisfunktion für die zukünftige sortimentspolitik besitzt.

Die weiteren Entscheidungsprobleme, die das Artikelniveau voraussetzen, treten in höher aggregierter Form auch als Entscheidungsprobleme auf Abteilungsniveau auf. Dabei ist das zur Entscheidungsfindung notwendige Vorgehen grundsätzlich auf den verschiedenen Aggregationsniveaus gleich. So ist z.B. im Rahmen der Plazierung zum einen die Regalplanung auf Artikelebene als auch die Geschäftsstättenplanung auf 
Abteilungsebene ein relevantes Problem. Für beide Probleme gelten aber analoge Lösungswege.

Insgesamt stellt sich die Kaufverbundanalyse auf der Basis von Kundenkarteninformationen als qualitative Weiterentwicklung eines Marktforschungsinstrumentes dar. Durch die differenzierte Datenbasis ergeben sich zum einen exaktere Ergebnisse als bei bisherigen Datenerhebungsmethoden. Zum anderen bieten sich durch personenspezifische Informationen weiterführende Analysegrundlagen. Demgegenüber eröffnet die Nachfrageverbundanalyse auf der Basis von Kundenkarteninformationen als Marktforschungsinstrument neue Analysemöglichkeiten. Hervorzuheben sind vor allem die Zeitraumorientierung dieses Instrumentes und die damit verbundene geringe Beeinflussung durch situative Aspekte.

Darüber hinaus ist allerdings festzustellen, daß die Verbundanalyse alleine keine umfassende Informationsbasis für den Handel darstellt. So kann sie z.B. nicht vollständig die Notwendigkeit von Experimenten im Handel beseitigen. 34 Dies ist vor allem durch die mangelhafte Berücksichtigung von Einflußgrößen auf das Entstehen von Verbundbeziehungen bedingt. Im Rahmen eines natürlichen Experiments können komplexe Wirkungen getestet werden. In diesem Zusammenhang ist die Verbundanalyse allerdings wiederum als Analyseinstrument für die im Rahmen des Experiments anfallenden Daten aufzuwerten.

Zusammenfassend ist somit die Verbundanalyse als ein Instrument der Informationsauswertung im Handel anzusehen. Ihre Ergebnisse stellen wiederum nur einen entscheidungsunter-

$34 \mathrm{Zu}$ Experimenten im Handel vgl. Ahlert, D., Günther, H., Strategisches Controlling und experimentelle optimierung im Handel, Arbeitspapier $\mathrm{Nr}$. 8 des Lehrstuhls für Betriebswirtschaftslehre, insbesondere Distribution und Handel der Westfälischen Wilhelms-Universität, 3. Aufl., Münster 1992 , S. 65 ff. 
stützenden Informationsgehalt dar. Durch die Anwendung sowohl zeitpunkt- als auch zeitraumbezogener verbundmessungsverfahren soll daher kein bestehendes Instrument des Handels ersetzt werden. Stattdessen stellt die Verbundmessung eine Erweiterung einer Vielzahl von Informationsanalyseverfahren des Handels dar.

\subsection{Anwendungsbereiche des Kaufverbundes im Rahmen des Han- delsmarketing}

\subsection{Die zusammensetzung von Geschäftseinheiten}

Die Geschäftsstättenplanung in Warenhäusern auf der Basis von Abteilungen ist in vielen Fällen sehr komplex. Um die Komplexität $z u$ reduzieren, werden Abteilungen $z u$ Obergruppen verdichtet. Auf diesem Aggregationsniveau erfolgen dann alle Planungsstufen einschließlich der wirtschaftlichkeitsrechnung. Lediglich in der letzten stufe, die zur Vorbereitung der Umsetzung dient, erfolgt wieder eine Aufteilung in $A b-$ teilungen. Die Obergruppen werden meist als Geschäftseinheit oder auch als Geschäftsfeld bezeichnet. ${ }^{35}$ Die in einer Geschäftseinheit zusammengefaßten Abteilungen sollen dabei aus Kundensicht einen geschlossenen Angebotskomplex darstellen. Somit entsteht kein Detailverlust durch die zusammenfassung.

In diesem zusammenhang sind nicht direkt die strategischen Geschäftseinheiten angesprochen, die z.B. bei der Portfolio-Planung genutzt werden, da für die einzelnen Geschäftseinheiten keine eigenständige strategische Ausrichtung existiert, obwohl dies grundsätzlich möglich ist. Vgl. Meffert, H., Marketing - Grundlagen der Absatzpolitik, a.a.0., s. 67. Auch der Begriff Geschäftsfeld dient hier nicht etwa einem strategischen Marktwahlprozeß. Vgl. ebenda, S. 96. Dies gilt um so mehr, da hier eine reine Abgrenzung nach Produkten erfolgt, die für eine strategische Ausrichtung unzureichend ist. Vgl. ebenda, S. 96; Köhler, R., Grundprobleme der strategischen Marketingplanung, in: Die Führung des Betriebs, Geist, M., Köhler, R. (Hrsg.), Stuttgart 1981, S. 261 ff. 
Als Beispiel seien an dieser stelle die Schuhabteilungen mit Herren-, Damen- und Kinderschuhen angeführt. Sie werden zur Geschäftseinheit Schuhe zusammengefaßt. Dies erscheint auf den ersten Blick plausibel. Betrachtet man jedoch den Kaufverbund, so ist festzustellen, daß die Schuhabteilungen unterschiedlichen clustern zugerechnet werden. Dabei war festzustellen, daß die Schuhe zusammen mit den jeweiligen Bekleidungssortimenten gekauft werden.

Diese Diskrepanz zwischen planerischer Zusammenfassung und Kaufverhalten stellt aus der sicht des Kaufverbundes eine falsche Verdichtung von Abteilungen $z u$ einem Geschäftsfeld dar. Für eine vollständige überprüfung der Zusammensetzung von Geschäftseinheiten aus sicht des Kaufverbundes werden die vom untersuchten Handelsunternehmen vorgegebenen Zuordnungen von Abteilungen $z u$ Geschäftseinheiten herangezogen. Diese werden mit den zuordnungen von Abteilungen $\mathrm{zu}$ Verbundclustern im Rahmen der Kaufverbundanalyse verglichen.

Im Rahmen dieser Analyse wird untersucht, ob die organisatorische und planerische systematisierung von Abteilungen bei der Bildung von Geschäftseinheiten das tatsächliche Kaufverhalten der Kunden widerspiegelt. Dazu wird eine Matrix aufgebaut (siehe Abbildung 26), die auf der einen Achse die vorgegebenen Geschäftseinheiten umfaßt und auf der anderen Achse die ermittelten Kaufverbundcluster. Die Abteilungen werden gemäß ihrer Zuordnung zu Geschäftseinheiten zu Clustern in die Felder der Matrix eingetragen.

Die cluster enthalten wesentlich mehr Abteilungen als die Geschäftseinheiten. Bei einer exakten Abbildung des Kaufverbundes durch die Bildung von Geschäftseinheiten können daher die Abteilungen mehrerer Geschäftseinheiten innerhalb desselben clusters liegen. Wenn aber nicht alle Abteilungen 


\begin{tabular}{|c|c|c|c|c|c|c|c|c|c|}
\hline $\begin{array}{l}\text { |Clumer } \\
\text { Goschättsboroich। }\end{array}$ & $\begin{array}{l}\text { Porsönlicher-/ } \\
\text { Grundbedarf }\end{array}$ & Spont & $\begin{array}{l}\text { Horron- } \\
\text { kleidung }\end{array}$ & Wäsche & $\begin{array}{l}\text { Damen- } \\
\text { kloidung }\end{array}$ & $\begin{array}{l}\text { Ein- } \\
\text { richtung }\end{array}$ & $\begin{array}{l}\text { Unterhaltungs- } \\
\text { haltungs- }\end{array}$ & $\begin{array}{l}\text { FuBbekl./Self- } \\
\text { made Mode }\end{array}$ & $\begin{array}{l}\text { Kinder- } \\
\text { Bodart }\end{array}$ \\
\hline DOB-Diverse & & & & & $\begin{array}{l}\text { Damenhü̈te } \\
\text { Mantel/Leder } \\
\text { Jeans/Marken } \\
\text { Kombimode } \\
\text { Kleider }\end{array}$ & & & & \\
\hline DOB-Chosen & & & & $\begin{array}{l}\text { Blusen } \\
\text { Da-Wollwaren }\end{array}$ & $\begin{array}{l}\text { Hosen } \\
\text { Röcke }\end{array}$ & & & & \\
\hline Ubrige DOB & & & & $\begin{array}{l}\text { Miedorwaron } \\
\text { Da-Tageswäsche } \\
\text { Da-Nachtwäsche }\end{array}$ & & & & & \\
\hline $\begin{array}{l}\text { FAKA-Gros. } \\
\text { konfoktion }\end{array}$ & & & $\begin{array}{l}\text { Mäntel/Leder } \\
\text { Men' Fashion }\end{array}$ & Anzügo/Hosen & & & & & \\
\hline HWKA-Chosen & & & & $\begin{array}{l}\text { Ho-Wollwaren } \\
\text { Ho-Artikel } \\
\text { Trikotagen }\end{array}$ & & & & & \\
\hline Knderbedart & & & & & & & & & $\begin{array}{l}\text { K-Wollwaren } \\
\text { Vorschulkleldung } \\
\text { Babyartikel } \\
\text { Kinderwälsche } \\
\text { Teenagerbeklold. } \\
\text { Kinderstrümple }\end{array}$ \\
\hline Schuhe & & & Horrenechuhe & & & & & $\begin{array}{l}\text { Damenechuthe } \\
\text { Hausschuthe }\end{array}$ & Kinderechuhe \\
\hline Strümpto/Handechuhe & & & & & & & & $\begin{array}{l}\text { Damenstrümpte } \\
\text { Horrenstrümpte } \\
\text { Handschuhb }\end{array}$ & \\
\hline Rostlicho Kolntextilion & & & & & & & & $\begin{array}{l}\text { Modeaccossolres } \\
\text { Kurzwaren } \\
\text { Handstrickgarne }\end{array}$ & \\
\hline Haush.wischo/Bottwaro & & & & & & Bottwaren & & HH-/Bottwäscho & \\
\hline Sport & & $\begin{array}{l}\text { Sommeraport otc. } \\
\text { Sportbekleidung } \\
\text { Sportartikel } \\
\text { Wintersport }\end{array}$ & & & & & & & \\
\hline Haushaltwaron & $\begin{array}{l}\text { Rund u.d. Küche } \\
\text { Fund u.d.Tisch } \\
\text { Waschmittel etc. }\end{array}$ & & & & & & & & \\
\hline Partúmento & Kosmelik & Körperpflege & & & & & & & \\
\hline Lederwaren/Schlirme & & $\begin{array}{l}\text { Schirme } \\
\text { Lederwaren }\end{array}$ & & & & & & & \\
\hline
\end{tabular}

Abb. 26: Zuordnungsvergleich von Abteilungen zu Geschäftsbereichen und Verbundclustern (Nur Geschäftsbereiche mit mehr als einer Abteilung) 
einer Geschäftseinheit innerhalb eines clusters liegen, so ergibt sich ein Hinweis auf eine strukturelle Unstimmigkeit.

Betrachtet man die unterschiedlichen zuordnungen, so zeigt sich eine hohe Überschneidung $z$ wischen den vorgegebenen Geschäftseinheiten und den ermittelten clustern. Allerdings ergeben sich einige bemerkenswerte Abweichungen vor allem in den Bekleidungsbereichen. Neben der angesprochenen Trennung von Damen- und Herrenschuhen fällt direkt eine Trennung im Herrenkleidungsbereich (Cluster 3 und 4 ) ins Auge. Innerhalb einer Geschäftseinheit werden deutlich unterschiedliche Zielgruppen zusammengefaßt. Daher sollte bei der Planung von Entscheidungen ein besonderes Augenmerk auf dieses Phänomen gerichtet werden. Dies gilt auch für andere Planungsaktivitäten innerhalb des Geschäftsbereiches, z.B. beim Einkauf, der die sortimentszusammensetzung bestimmt.

Im Bekleidungsbereich zeigt sich darüber hinaus ein weiterer struktureller Aspekt. Dieser betrifft die deutliche Bildung eines "Wäsche-Clusters". Dabei trennt der Kaufverbund deutlich sowohl im Herren- als auch im Damenbekleidungsbereich die Wäscheabteilungen von den anderen Bekleidungsbereichen und faßt sie in einem Cluster zusammen. Dies zeigt, daß die Wäschekäufe innerhalb eines Haushaltes offensichtlich im Rahmen desselben Einkaufs, wahrscheinlich von nur einer Person sowohl für den Damen- als auch den Herrenbedarf getätigt werden. Daher ist bei der Geschäftsstättenplanung unabhängig von der bisherigen zugehörigkeit zu einer Geschäftseinheit eine Zusammenfassung dieser Abteilungen zu einer räumlichen Einheit anzustreben.

Die vergleichende Betrachtung der Kaufverbundcluster dieser Geschäftsstätte mit vorgegebenen Geschäftseinheiten zeigt strukturelle Schwächen der Geschäftseinheiten vor allem im Bekleidungsbereich auf. Diese sollten bei der Geschäftsstättenplanung berücksichtigt werden. Wenn die gleichen zuord- 
nungsprobleme auch bei anderen Geschäftsstätten auftreten, sollte das Handelsunternehmen grundsätzlich die Umstrukturierung der betroffenen Geschäftseinheiten in Betracht ziehen.

Grundsätzlich ist eine solche Analyse in gleicher Weise auch für die Zusammenführung von Artikeln zu Artikelgruppen oder von Artikelgruppen zu Hauptartikelgruppen etc. auf jedem Aggregationsniveau durchführbar. In diesem zusammenhang sind jedoch zwei Aspekte problematisch. Dazu gehört zum einen der enorme Datenumfang, der für eine detaillierte Analyse auf Artikelebene notwendig ist. Zum anderen können aufgrund der Komplexität der Matrizen sowie dem daraus resultierenden technischen Aufwand jeweils nur relativ kleine Ausschnitte des Sortiments betrachtet werden.

\subsection{Plazierungsentscheidungen auf der Basis von Kaufver- bundbeziehungen}

Die Plazierung wird im allgemeinen als ein Entscheidungsproblem auf der Artikelebene betrachtet. Dies gilt auch für die bisherige Einbeziehung der Verbundforschung. 36

Im Rahmen dieser Untersuchung, die auf den Kaufverbundbeziehungen zwischen Abteilungen aufbaut, wird die Plazierung weiter gefaßt. Sie ist auf die räumliche Anordnung einzelner Abteilungen im zusammenhang mit der Gesamtkonzeption einer Geschäftsstätte ausgerichtet. Dabei stellt wiederum die clusteranalyse der Kaufverbundstrukturen ein entscheidendes Informationspotential dar. Allerdings wird in diesem zusammenhang zur Analyse nicht nur die reine Zuordnung einzelner Abteilungen $z u$ den clustern herangezogen. Vielmehr wird zu-

Vgl. Müller-Hagedorn, L., Heidel, B., Plazierungspolitik nach dem Verbundkonzept im stationären Einzelhandel, a.a.O., S. $19 \mathrm{ff}$. 
sätzlich die Intensität der Verbundbeziehungen in die Betrachtung aufgenommen. Diese Intensitäten sind aus dem Dendrogramm der clusteranalyse ablesbar. Es ergeben sich grundsätzlich drei Gruppen von Verbundbeziehungen: 37

- Die Kerngruppen

- Die "normale" Verbundbeziehung

- Die Einzelabteilungen oder Randgruppen

Als Beispiele für Kerngruppen seien an dieser stelle die Abteilungen Kleinelektro, Radio-Fernsehen und Lampen aus dem Elektronikcluster und die Abteilungen Haushaltswäsche, Bettwäsche und Kurzwaren aus dem cluster Wäsche angeführt. Ein Beispiel für eine Einzelabteilung z.B. die Damenhüte aus dem Bereich Damenmode. 38 Die "normalen" Verbundbeziehungen umfassen alle Intensitäten zwischen diesen Extremausprägungen.

Bei einer Plazierungsplanung sollten diese Informationen unter den restriktiven Nebenbedingungen der vorhandenen Verkaufsflächen und ihres zuschnittes berücksichtigt werden. Auch in diesem Fall ist grundsätzlich wieder der allgemeine Kaufverbund heranzuziehen.

Bei der Planung der Position der Abteilungen im Rahmen eines Gesamtkonzeptes zur Geschäftsstättengestaltung bilden die ermittelten Kerngruppen Abteilungsgruppierungen, die aus sicht des Nachfrageverbundes nicht getrennt positioniert werden sollten.

37 Vgl. Kapitel B.2. dieser Untersuchung.

38 Eine detaillierte Beschreibung der empirischen Ergebnisse ist Kapitel C.2.1 dieser Untersuchung $z u$ entnehmen. 
Die Randabteilungen oder Randgruppen stellen hingegen eher ein Flexibilitätspotential bei der Geschäftsstättenplanung dar. Zwar sollten auch diese Abteilungen zusammen mit dem cluster, dem sie zugeordnet sind, positioniert werden, doch wenn dies nicht $z u$ realisieren ist, werden damit auch keine intensiven bestehenden Kaufverbundbeziehungen zerstört. Diese Abteilungen stellen zumeist zielkaufabteilungen dar, die z.B. auch bei der Eliminierungsentscheidung eher geringe wirkungen für die restlichen Abteilungen hervorrufen.

zielkaufabteilungen stellen allerdings trotz der fehlenden Verbundbeziehungen ein wichtiges Potential für die Handelsunternehmen dar. Wenn sie richtig plaziert werden, tragen sie dazu bei, die Konsumenten auch in ansonsten kaum frequentierte Bereiche der Geschäftsstätte zu führen. Dazu können sie z.B. am Ende eines Gangsystems oder in den oberen stockwerken plaziert werden. Dadurch wird die "Kontaktstrecke" des Kunden mit dem restlichen sortiment erhöht. Dies führt zu einer erhöhten Wahrscheinlichkeit für weitere Käufe oder zumindest beim Konsumenten zu einer besseren Kenntnis des Gesamtsortiments.

\subsection{Auswirkungen der Eliminierung von Abteilungen}

Eine der zentralen Fragen bei der Neuplanung einer Geschäftsstätte ist, welche Auswirkungen es hat, wenn eine bestimmte Abteilung eliminiert wird. Für eine Bewertung der Auswirkungen der Eliminierung einer bestimmten Abteilung ist es jedoch nicht ausreichend, den wegfallenden Umsatz dieser Abteilung gegen einen an Erfahrungswerten orientierten $\mathrm{zu}-$ satzumsatz anderer Abteilungen, die auf dieser freigewordenen Fläche entstehen oder vergrößert werden, aufzurechnen.

Zum einen ist $z u$ berücksichtigen, daß es bestimmte Abteilungen gibt, die ein Kunde bei einem Vollsortimenter erwartet 
und die überwiegend auch als sogenannte Frequenzbringer dienen. Bei der Aufgabe einer Abteilung ist daher zumindest die Möglichkeit eines Frequenzverlustes einzukalkulieren. zum anderen besteht ein Kaufverbund zwischen der aufzulösenden Abteilung und allen anderen Abteilungen der Geschäftsstätte. Der auslösende Faktor des Verbundkaufes ist zwar bei einem symmetrischen Verbund nicht $z u$ bestimmen. Durch die Auflösung einer Abteilung werden jedoch in jedem Fall Kaufverbundbeziehungen zerstört. Daher ist auch ohne die Feststellung eines verbundkaufauslösenden Produktes eine Umsatzeinbuße bei Abteilungen mit starken Kaufverbundbeziehungen zur eliminierten Abteilung anzunehmen.

Insgesamt müssen zur Quantifizierung eines wirtschaftlichen Effektes die folgenden Aspekte berücksichtigt werden:

- Umsatzwegfall der aufgelösten Abteilung,

- Umsatzeinbuße bei den Abteilungen mit starken Kaufverbunden zur aufgelösten Abteilung,

- Umsatzeinbußen durch Frequenzverlust im Gesamthaus,

- Umsatzgewinn durch die Vergrößerung bzw. Neuaufnahme anderer Abteilungen.

Für die Entscheidungsfindung ist dies jedoch noch nicht ausreichend. So sollte zumindest bis zur stufe des Deckungsbeitrages weitergerechnet werden. Die Deckungsbeitragsrechnung ist aber völlig unabhängig von unterschiedlichen Verbundbeziehungen der Abteilungen. Zudem sind z.B. die Gewinnspannen der produkte unternehmensspezifisch unterschiedlich. Daher wird diese Rechnungsstufe bei den Darstellungen nicht berücksichtigt.

Mögliche positive Auswirkungen auf den Umsatz vergrößerter Abteilungen durch einen Gewinn in der sortimentskompetenz und eventuell der größeren Akzeptanz durch die Kunden werden hier nicht zusätzlich berücksichtigt, da sie in den Erfah- 
rungswerten für die Umsätze auf zusätzlichen (vergrößerten) Verkaufsflächen implizit enthalten sind. Auch grundsätzliche positive Effekte durch den Neuerungseffekt sollen an dieser Stelle nicht einbezogen werden, da sie eher auf die gesamte Geschäftsstätte wirken und nicht direkt durch die Auflösung einer Abteilung ausgelöst werden.

Insgesamt ergibt sich also das folgende Berechnungsmodell für die Umsatzwirkung:

$$
\Delta U_{X}=\sum_{i=1}^{n}\left(E_{i} \cdot \Delta \mathrm{qm}_{i}\right)-M\left(U_{t}\right)-\sum_{j=1}^{n}\left(V U_{j} \cdot P_{y}\right)-U_{G} \cdot P_{z}
$$

wobei :

$\Delta \mathrm{U}_{\mathrm{X}}=$ Umsatzwirkung der Auflösung der Abteilung $\mathrm{x}$

$E_{i}=$ Umsatz-Erfahrungswert eines zusätzlichen qm in Abt. i

$\Delta \mathrm{gm}_{\mathrm{i}}=$ Anzahl zusätzlicher Quadratmeter für Abteilung $i$

$\mathrm{VU}_{j}=$ Verbundumsatz von Abt. $j$ und aufgelöster Abteilung

$\mathrm{P}_{\mathrm{y}} \quad=$ Prozent Umsatzeinbuße in Verbundintensitätsgruppe $\mathrm{y}$

y = Verbundintensitätsgruppe (starker/schwacher Verbund)

$\mathrm{U}_{\mathrm{G}} \quad$ Umsatz der gesamten Geschäftsstätte

$P_{z} \quad=$ Prozentsatz $z$ der Umsatzeinbuße in der gesamten Geschäftsstätte, die durch die Abteilungsauflösung entsteht (z.B. durch Frequenzverluste)

$M\left(U_{t}\right)=$ Umsatzmittelwert der aufgelösten Abteilung der letz. ten $t$ Jahre

Für eine Quantifizierung der Modellgrößen sind einige Operationalisierungen erforderlich:

- Der Umsatzwegfall der aufgelösten Abteilung ist aus den letzten Umsatzzahlen abzuleiten. Um einen besseren wert 
zu erhalten, kann ein Mittelwert der Umsätze z.B. der letzten drei Jahre herangezogen werden.

- Bei der Berechnung der Umsatzeinbuße bei den Abteilungen mit starken Kaufverbunden zur aufgelösten Abteilung ist eine Vorstufe der clusterbildung heranzuziehen. In der Profilanalyse werden für jede Abteilung ihre Verbundkäufe mit anderen Abteilungen ermittelt. Im Rahmen dieser Arbeit ist dies anhand von Absatzzahlen durchgefürt worden. Aus der Anzahl der Verbundbeziehungen kann die Intensität des Verbundes abgeleitet werden. Durch die analoge Vorgehensweise auf der Basis von Umsatzzahlen ergibt sich eine monetäre Ausprägung der Verbundbeziehungen. Die wertmäßige Höhe der Verbundkäufe hilft bei der Quantifizierung des Verlustes.

Eine Möglichkeit zur Messung des Verlustes ist die Beurteilung der Verbundabteilungen nach der Intensität ihrer Verbundbeziehungen zur aufzulösenden Abteilung z.B. in drei Gruppen. Dabei entstehen Abteilungsgruppen mit starken, mittleren und schwachen Verbundbeziehungen. Jeder dieser Gruppen wird dann ein Prozentwert zugeordnet, der mit dem umsatzdefinierten Verbund multipliziert wird. Der resultierende wert ergibt dann den Verbundumsatzverlust pro Verbundabteilung. Durch summenbildung ergibt sich dann die gesamte Umsatzeinbuße durch Verbundverluste.

- Die Umsatzeinbuße durch Verluste in der Gesamtfrequenz wird ebenfalls durch die Annahme eines Prozentsatzes ermittelt. Dieser Prozentsatz bezieht sich dann auf den Gesamtumsatz des Hauses. Die Höhe dieses Prozentsatzes orientiert sich an der Gesamtstärke der Verbundbeziehungen der aufzulösenden Abteilung. Je größer die Anzahl der Verbundbeziehungen der zu eliminierenden Abteilung, desto höher ist der Verlust in der Gesamtfrequenz. Zum Beispiel führt die Aufgabe einer Lebensmittelabteilung, die die 
mit Abstand größte Anzahl an Verbundbeziehungen aufweist, $z u$ einem größeren Frequenzverlust als die Eliminierung der Möbelabteilung, die nur wenige Verbundbeziehungen aufweist.

- Der Umsatzgewinn durch die Nutzung der bei der Auflösung einer Abteilung freiwerdenden Verkaufsfläche durch andere Abteilungen kann vor allem durch die Anwendung von Erfahrungswerten des Handelsunternehmens in seiner Höhe bestimmt werden. Diese Erfahrungswerte sind je nach Abteilung unterschiedlich. Wird die freiwerdende Verkaufsfläche auf mehrere andere Abteilungen verteilt, so ist der Gesamtgewinn ebenfalls durch Summenbildung festzustellen.

Der Saldo der vier oben genannten Werte ergibt dann die gesamte Umsatzwirkung der Auflösung einer Abteilung. Problematisch stellt sich in diesem Zusammenhang vor allem die Festlegung der Prozentsätze für die Ermittlung der Verbundverluste der einzelnen Abteilungen dar. Für eine realistische Annahme bzgl. der Prozentsätze sind zum einen fundierte Hypothesen $z u$ bilden und zum anderen Erfahrungswerte aus früheren Eliminierungen heranzuziehen. Darüber hinaus sind Rechnungen mit unterschiedlichen prozentsätzen durchzuführen. Dabei sollte zumindest eine Version mit sehr optimistischen und eine mit pessimistischen Annahmen berechnet werden. Dies zeigt dem Handelsunternehmen den Bereich möglicher Auswirkungen der Eliminierungsentscheidung auf.

Mit dem vorgestellten, relativ einfachen und robusten Modell zur Berechnung der Umsatzwirkungen ergibt sich ein solides Instrument zur Ermittlung einer ersten stufe der Entscheidungsunterstützung. Dabei zeigt sich, daß mit der Nutzung der Ergebnisse der Profilanalyse ebensogut gearbeitet werden kann wie mit der endgültigen clusterbildung. Somit können unterschiedliche stufen der Ermittlung eines systems von 
Kaufverbunden für die Marketingarbeit in Handelsunternehmen genutzt werden.

\subsection{Raufverbundbeziehungen als Grundlage zur Ermittlung von Rundenwegen in der Geschäftsstätte}

Eine völlig andere Möglichkeit der Nutzung von Kaufverbunddaten ist die Messung der Kundenbewegungen innerhalb der Geschäftsstätte. Diese Kundenbewegungen waren bisher nur durch Beobachtung oder Befragung im Rahmen von Kundenlaufstudien $\mathrm{zu}$ erfassen. Dieses relativ teure Instrument kann durch eine neuartige Nutzung von Kaufverbunddaten $z . T$. ersetzt werden. Dazu ist allerdings z.T. ein anderes empirisches Vorgehen erforderlich als bei der Ermittlung der Kaufverbundstrukturen.

Schon im Grundsatz ist der symmetrische verbund, der in dieser Untersuchung postuliert wurde, durch eine quasi-asymmetrische Kaufverbundauffassung $z u$ ersetzen, indem eine Richtung zwischen zwei Käufen festgelegt wird. Die Verbundauffassung ist dabei nur "quasi-"asymmetrisch, weil zwar die Identität zwischen Kaufgut A und Kaufgut B gleich Kaufgut B und Kaufgut $A$ aufgehoben wird. Diese Identität wird aber nicht ersetzt durch eine kausale Beziehung zwischen den Käufen der Güter, wobei ein Gut den auslösenden Faktor darstellt. Die Richtung zwischen den Käufen zweier Güter wird allein durch den zeitpunkt des Kaufs bestimmt. Alle Einzelkäufe innerhalb eines Kaufaktes werden nach ihrer zeitlichen Abfolge geordnet. Die Interpretation eines solchen einzelnen Kaufaktes zeigt Abbildung 27.

Die auf diese weise vorbereiteten Daten werden dann analog zur bisherigen Aufbereitung von Kaufverbunddaten in eine Matrix eingetragen, wobei aufgrund der Asymmetrie die gesamte Matrix mit Daten gefüllt wird. Die Abteilungen, die die Zei- 


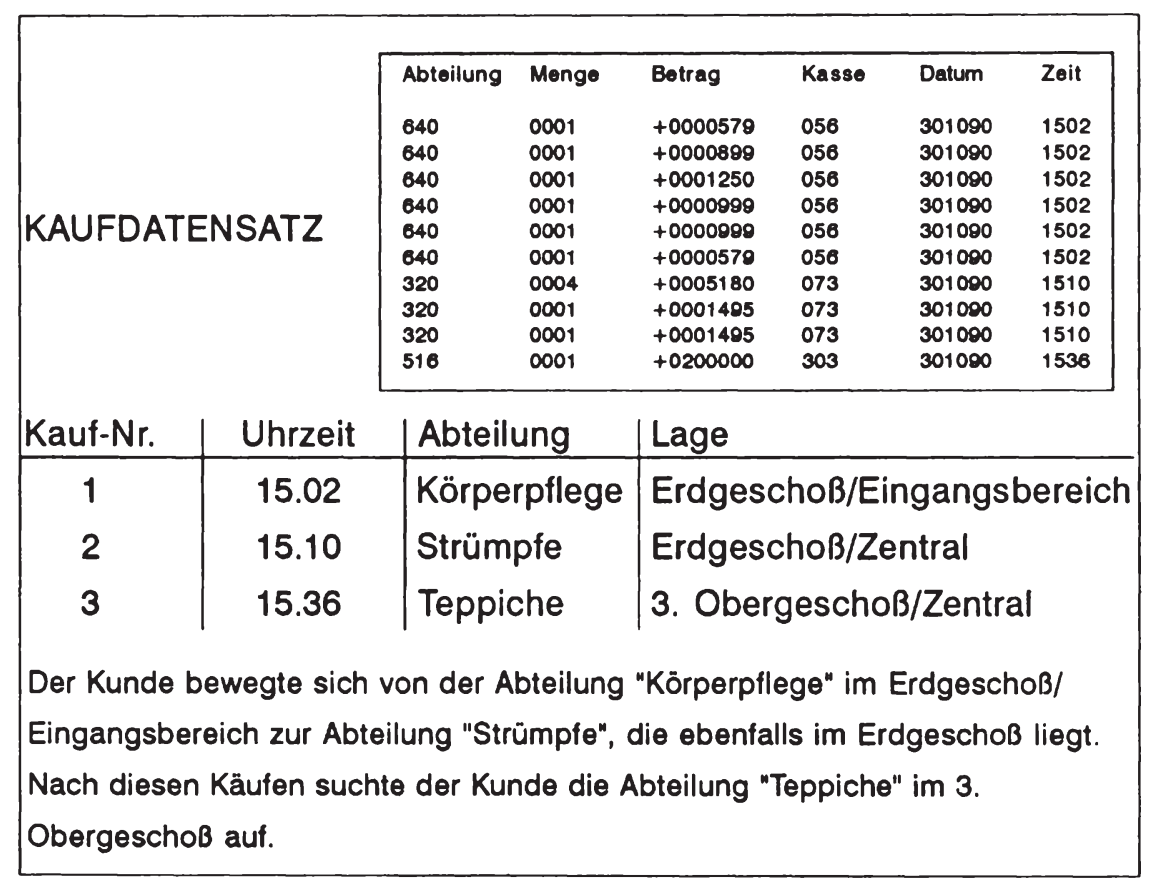

Abb. 27: Ermittlung eines Kundenweges anhand eines Kaufdatensatzes

len und spalten bestimmen, sollten in diesem Fall direkt nach ihrer Plazierung in Etagen geordnet werden. Auf diese Weise können sehr leicht alle Bewegungen innerhalb eines stockwerkes und auch zwischen stockwerken identifiziert werden, da sie jeweils innerhalb der Matrix durch komplexe rechteckige Ausschnitte bestimmt sind. Abbildung 28 zeigt ein Beispiel für eine derartige Matrix.

Dabei werden die Kaufakte in Kaufpaare zerlegt und mit dem wert 1 in die Matrix eingetragen. Anhand der absoluten Zahlen in der Matrix ist nun festzustellen, wie häufig sich ein Kunde von einer Abteilung aus zur anderen bewegt hat. In den spalten der Matrix stehen alle Käufe, bei denen nach dem Kauf in dieser Abteilung noch andere Käufe getätigt werden 
(von Abt. A nach Abt. X). In einer Zeile der Matrix stehen alle Käufe, bei denen der Konsument vor dem Kauf in dieser Abteilung noch in anderen Abteilungen gekauft hat (von Abt. $\mathrm{X}$ nach Abt. A).

\begin{tabular}{|c|c|c|c|c|c|c|c|c|c|}
\hline VON & $\begin{array}{c}A B T A \\
\text { UG }\end{array}$ & $\begin{array}{c}\text { ABT } B \\
\text { UG }\end{array}$ & $\begin{array}{c}A B T C \\
\text { UG }\end{array}$ & $\begin{array}{c}\text { ABT D } \\
\text { EG }\end{array}$ & $\begin{array}{c}\text { ABT } E \\
E G\end{array}$ & $\begin{array}{c}A B T F \\
E G\end{array}$ & & $\begin{array}{l}A B T Z \\
4.0 G\end{array}$ & $\Sigma$ \\
\hline $\begin{array}{c}\text { ABT } \mathrm{A} \\
\text { UG }\end{array}$ & 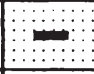 & 616 & 917 & 683 & 626 & 312 & & 212 & \multirow{7}{*}{ 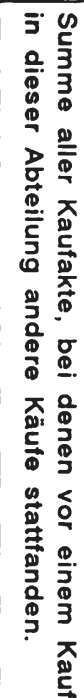 } \\
\hline $\begin{array}{c}\mathrm{ABT} \\
\cup \mathrm{U}\end{array}$ & 327 & 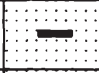 & 359 & 576 & 283 & 751 & & 131 & \\
\hline $\begin{array}{c}\mathrm{ABT} C \\
\text { UG }\end{array}$ & 347 & 465 & 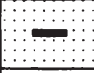 & 236 & 529 & 579 & & 106 & \\
\hline $\begin{array}{c}\text { ABT D } \\
\text { EG }\end{array}$ & 293 & 733 & 412 & חדיח: & 316 & 822 & & 231 & \\
\hline $\begin{array}{c}\mathrm{ABT} \\
\mathrm{EG} \\
\end{array}$ & 421 & 183 & 451 & 483 & 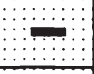 & 671 & & 172 & \\
\hline $\begin{array}{c}\mathrm{ABT} F \\
\mathrm{EG}\end{array}$ & 736 & 229 & 679 & 536 & 279 & - & & 43 & \\
\hline $\begin{array}{l}\text { ABT } Z \\
4.0 G \\
\end{array}$ & 236 & 139 & 145 & 244 & 117 & 305 & & - & \\
\hline & $\begin{array}{l}\text { Summ } \\
\text { dieser }\end{array}$ & $\begin{array}{l}\text { aller } K \\
\text { Abteilun }\end{array}$ & $\begin{array}{l}\text { aufakte } \\
\text { ig statt }\end{array}$ & $\begin{array}{l}\text { bei de } \\
\text { anden. }\end{array}$ & nen no & ch Kăuf & nach einem & uf in & \\
\hline
\end{tabular}

$U G=$ UntergeschoB; $E G=$ ErdgeschoB; $O G$ = ObergeschoB

Abb. 28: Häufigkeitsmatrix der Kundenbewegungen

Bei einer spaltenweisen Addition der Werte ergibt sich die Summe aller Verbundkäufe, die nach einem Kauf in einer Abteilung noch getätigt wurden. Dies ist gleichzusetzen mit der Bedeutung einer Abteilung als im Verbundkauf zeitlich vorgelagerte Abteilung. Analog ergibt sich bei zeilenweiser Addition die Summe aller Verbundkäufe, in denen vor dem Kauf in einer Abteilung noch andere Käufe getätigt wurden, also 
die Bedeutung als im Verbundkauf zeitlich nachgelagerte Abteilung.

Mit den auf diese Weise aus den Kaufverbunddaten ermittelten Kundenströmen ist es möglich, z.B. eine Grundlage für die Bewertung der Lage unterschiedlicher Bereiche der Verkaufsfläche innerhalb einer Geschäftsstätte vorzunehmen. Eine solche Bewertung ist wiederum eine nützliche Zusatzinformation für die Planung von Plazierungen, die dann dazu beitragen können, den Kundenstrom gezielt durch eine Geschäftsstätte zu leiten.

Für diese Anwendung von Kundenkarteninformationen im Rahmen von Verbundanalysen ist anzumerken, daß gewisse systemimmanente Schwachpunkte gegeben sind, die zu beachten sind, um keine Fehlinterpretation der erarbeiteten Ergebnisse zuzulassen. So ist festzustellen, daß zwar die Nutzung der Kaufdaten eine sehr objektive Erfassung im Vergleich zur Beobachtung oder Befragung ermöglicht, sie diese jedoch nicht ersetzen kann. Die Befragung und insbesondere die Beobachtung liefern wesentlich umfangreichere Erkenntnisse über den gesamten Kaufvorgang, wohingegen bei der Nutzung der Kundenkartendaten keine Informationen vorhanden sind:

- über eventuelle Beratungsgespräche (Beobachtung/Befragung),

- über den Weg, den der Kunde zwischen zwei Käufen innerhalb der Geschäftsstätte zurückgelegt hat, bzw. welche Abteilungen er besucht hat (Beobachtung),

- über Verweildauern an bestimmten Punkten der Geschäftsstätte bzw. keine Beschreibung seines suchverhaltens (Beobachtung), 
- über äußere Ablenkungen wie z.B. private Gespräche etc. (Beobachtung),

- über Beweggründe, Informationsverhalten etc. des Kunden (Befragung).

Stattdessen kann mit Hilfe der Kundenkarten sehr präzise aufgezeichnet werden, was der Kunde tatsächlich gekauft hat. Bei einer Analyse auf Artikelebene, die z.B. die Käufe innerhalb einer Abteilung analysiert, ergeben sich wesentlich detailliertere Informationen, die präzise besuchte Punkte innerhalb einer Abteilung angeben können, wenngleich auf diesem Aggregationsniveau keine Ermittlung einer zeitlichen Abfolge mehr möglich ist.

\subsection{Anwendungsbereiche des Nachfrageverbundes im Rahmen des Handelsmarketing}

\subsection{Implikationen für die Rommunikationspolitik im Rahmen eines Data-Base-Marketing}

Im Zusammenhang mit der zunehmenden Informationsüberlastung der Konsumenten ${ }^{39}$ durch Werbung per Anzeigen und Fernsehspots entwickelte sich in den vergangenen Jahren die Direktwerbung zunehmend $z u$ einem relevanten Marketinginstrument. Mit Hilfe der gezielten, persönlichen Ansprache der Konsumenten sollen Streuverluste der Werbung minimiert und die wirkung erhöht werden, da sich Kontaktwahrscheinlichkeit und -qualität verbessern. Die stationären Handelsunternehmen waren bei der Möglichkeit, die Direkt-Werbung als Medium zu nutzen, benachteiligt. Ihnen fehlten die relevanten Basisinformationen, da weder Namen- noch Adressdaten vorlagen. In

$39 \mathrm{Vgl}$. Kroeber-Riel, W., Informationsüberlastung durch Massenmedien und Werbung in Deutschland, Messung - Interpretation - Folgen, in: DBW, Heft $3 / 1987$, S. $257 \mathrm{ff}$. 
Ausnahmefällen vorliegende Daten aus Auftragslisten oder Beschwerdebüchern waren nicht umfangreich genug, um eine ausreichende Basis $z u$ bilden.40 Ein ungezielter Ankauf von Adressdateien schafft für das Problem keine Abhilfe, da das Kundensegment des Handels nicht oder nur unzureichend abgebildet wird und somit wiederum eher unspezifische, wenn auch persönliche Werbebotschaften entstehen.

Die situation wurde mit der zunehmenden Verbreitung von Direct-Mailing Aktivitäten verschärft. Derartige persönlich adressierte, aber in ihrem Inhalt unspezifische und häufig für den Adressaten irrelevante Informationen überschwemmten den Konsumenten. Sie entwickelten sich $\mathrm{zu}$ einem weiteren Informationsüberlastungsproblem, bei dem die einzelne Werbebotschaft kaum noch wirkt. Um diesem Problem zu begegnen, sollten die Direkt-Werbungen vor allem in ihrer Angebotsgestaltung präzise auf den jeweiligen Adressaten zugeschnitten werden. Für den Handel ergeben sich durch die Ausgabe von Kundenkarten Chancen, beide Probleme, die Datenbeschaffung im Adressbereich ${ }^{41}$ und die Spezifizierung der Angebote, zu lösen. Dabei hat der Handel durch die vorliegenden Kaufdaten der Kunden sogar einen entscheidenden Vorteil gegenüber anderen Werbungtreibenden. So besteht die Möglichkeit, die Direktwerbung auch für den Handel als qualifiziertes Kommunikationsinstrument zu nutzen.

Im Zusammenhang mit der Aufbereitung der mit Hilfe der Kundenkarte ermittelten Kaufdaten stellt die Verbundanalyse ein wichtiges Instrument dar. Da der Kaufverbund stark situativ beeinflußt und an den räumlichen Gegebenheiten orientiert ist, ist in diesem Falle der Nachfrageverbund von besonderem

40 Vgl. Poggenpohl, M., Kundenkarteninformationen als Instrument der Verbundanalyse im Einzelhandel, a.a.0., s. 54 .

41 o.V., Ihr Grundstein für eine treffsichere Direktwerbung, in: ehb, $1 / 91$, S. $42 \mathrm{ff}$. 
Interesse. Da im Nachfrageverbund Kaufintensitätsgruppen, die sich bei der empirischen Analyse als sehr stabil erwiesen haben, gebildet werden, kann anhand der Ergebnisse festgestellt werden, welche der Abteilungen (oder auf der Basis von Artikeldaten, welche Artikel) von den Konsumenten besonders stark genutzt werden, woraus ein erhöhter Interessewert ableitbar ist.

Durch die Ermittlung dieser Kombinationen innerhalb spezifischer soziodemographischer Gruppen, deren Nachfrageverbund zum Teil - gemessen an den Intensitäten nicht an den Grundmustern - erheblich divergiert, ist eine spezifische Ansprache möglich. Somit ist die Möglichkeit gegeben, die relevanten Abteilungen für bestimmte zielgruppen herauszufiltern. Aus diesen Informationen ist es daraufhin möglich, zielgruppenspezifische Angebotszusammenstellungen abzuleiten. Für andere Warengruppen dagegen, die für dieselbe Gruppe nicht relevant sind, werden bzgl. der Gestaltung von Direktwerbung Sperrvermerke gesetzt, so daß der Umfang der Direktmarketing-Werbung reduziert und gleichzeitig der Inhalt auf die Interessen der Kunden zugeschnitten wird.

In diesem Zusammenhang ist noch $z u$ erwähnen, daß die Informationsbasis über die einzelnen Kundenkartenbesitzer sukzessive verbessert werden kann, indem im Umfeld jeder Direktwerbungsaktion die Reaktionswerte ermittelt werden. 42 Dabei kann die Kundenkarte weitgehend auf klassische Responseelemente, die der Direktwerbung beigefügt werden, verzichten, da jederzeit der Kauf beworbener Artikel bzw. in beworbenen Abteilungen anhand der Kaufdaten überprüft werden kann.

42 Vgl. Kreuzer, R.T., Weppler, K., Marketing by Database. Eine Herausforderung für Markenartikler, in: Markenartikel 10/1990, S. $493 \mathrm{ff.;} \mathrm{O.V.,} \mathrm{Das} \mathrm{Raster} \mathrm{wird} \mathrm{feiner,}$ in: Textilwirtschaft, Nr. 9/1989, S. 32. 
Darüber hinaus bietet sich die Möglichkeit, sowohl die Eignung der beworbenen Ware zur Belebung der Käufe auch in anderen Abteilungen $z u$ ermitteln als auch weitere Informationen über das Kaufverhalten der Konsumenten zu gewinnen. Dazu wird der spezifische Kaufverbund des Konsumenten in bezug auf die beworbene Ware festgestellt und als zusätzliche Information gespeichert. Zum einen wird dadurch die Kenntnis des Handelsunternehmens bezüglich der Interessenlage der Konsumenten verbessert. Zum anderen kann die Frequenzwirkung bestimmter waren im Zusammenhang mit werbeaktionen festgestellt werden. Auf diese Weise kann die Direktwerbung auch aus sicht des Handelsunternehmens optimiert werden, da dieses besonders verbundintensive Produkte im Rahmen ihrer Werbung aufgrund der verkaufsfördernden Wirkung bevorzugt.

Ein weiterer wichtiger Aspekt, bei dem der Nachfrageverbund entscheidenden Einfluß auf die Kommunikationsarbeit und insbesondere das Direktmarketing des Handels haben kann, ist die Bestimmung von Zielgruppen. Bisher wurden die Zielgruppen für Direktwerbungsaktionen weitgehend aus den Personendaten, die dem Unternehmen vorlagen, abgeleitet. Dabei konnte zwar eine gewisse Verhaltensrelevanz der Kriterien unterstellt werden. Aber weder Quantität noch Qualität der Informationen genügt den Anforderungen eines qualifizierten Direktmarketing.

Eine wesentlich präzisere Abbildung des Bedarfsmusters bestimmter Kundengruppen ermöglicht die Analyse ihrer Kaufdaten. ${ }^{43}$ In diesem Zusammenhang fallen bei der Zielgruppenbildung auch Informationen über die Kaufintensitäten der ermittelten Gruppen in bestimmten Abteilungen oder auf Artikelniveau bei bestimmten Artikeln an. Damit ist direkt eine $\mathrm{Zu}-$ sammenstellung von Werbeartikeln ableitbar, die der besonderen Interessenlage der jeweiligen Gruppe entspricht. 
Insgesamt zeigt sich, daß mit Hilfe der Nutzung der Nachfrageverbundinformationen im Rahmen der Kommunikationsarbeit eines Handelsunternehmens eine Möglichkeit besteht, ein qualifiziertes Direktmarketing aufzubauen. Dies hat große Wirkungschancen beim Kundenkartenbesitzer und darüber hinaus auch bei Kundengruppen mit ähnlicher demographischer struktur, die z.B. aus Adressdateien gewonnen werden können. Obwohl die Kundenkartenbesitzer keine repräsentative stichprobe abbilden, ist eine Anwendung der Analyseergebnisse auf Nicht-Kartenbesitzer möglich. Dies ergibt sich aus der starken Annäherung des Nachfrageverbundes an den Bedarfsverbund. Der Bedarfsverbund kennzeichnet eine von der Geschäftsstätte unabhängige Größe. Die ermittelten strukturen können daher als grundlegend für Konsumenten mit gleichen demographischen strukturen angesehen werden.

\subsection{Implikationen für die sortimentspolitik}

Ein zentrales Profilierungsinstrument im Einzelhandel stellt die sortimentspolitik dar. 44 sie soll inhaltliche, räumliche und zeitliche Nachfragesynergien schaffen, die zu einer Verbesserung der Wettbewerbsposition, $z u$ einer Alleinstellung und letztlich zur sicherung der Ertragslage des Handelsunternehmens führen. Diese Auffassung der Sortimentspolitik als Auslöser von Nachfragesynergien impliziert direkt die Anwendbarkeit von Verbundanalysen als Informationsinstrument. Da die sortimentspolitik zumindest mittelfristig stabil sein muß, ist der Kaufverbund als situatives Konstrukt allerdings weniger geeignet. Stattdessen werden auch für

44 Zum Sortiment als zentraler Bestandteil einer strategischen Erfolgsposition vgl. Meffert, H., Erfolgsfaktoren im Einzelhandelsmarketing, in: Marketing Erfolgsfaktoren im Handel, Bruhn, M. (Hrsg.), Frankfurt/Main, New York 1987 , S. $20 \mathrm{f}$. 
diese Problemstellung die Ergebnisse des Nachfrageverbundes herangezogen.

Betrachtet man die drei Grundtypen von Sortimentsstrategien, die z.B. Warengruppen oder Abteilungen betreffen, so ist zwischen Expansions-, Konsolidierungs- und Schrumpfungsstrategien $z u$ unterscheiden. 45 Der Nachfrageverbund als Dokumentation des vorherrschenden Kaufverhaltens kann allerdings nur sehr bedingt bei der Entscheidung bzgl. einer Expansionsstrategie behilflich sein, da er eine reine ex post Betrachtung darstellt, die nur vor dem Hintergrund der aktuellen Situation auch prognostische Bedeutung besitzt. Nur wenn die Ergebnisse einer Geschäftsstätte auf eine andere übertragen werden können, die z.B. über eine Ausweitung des bestehenden Sortiments im Hinblick auf eine fehlende Abteilung, die bei der ersten vorhanden ist, nachdenkt, bieten sich Ansatzpunkte für eine Nutzung im Rahmen einer Expansionsplanung.

Für eine Eliminierungsentscheidung im Rahmen einer Schrumpfungsstrategie bieten die Verbundanalysen jedoch detaillierte Informationen. Neben der Überprüfung der isolierten wirtschaftlichen situation einer Abteilung und ihrer Bedeutung im Wettbewerbsumfeld ist vor allem auch ihre Bedeutung im Kontext der gesamten Sortimentspolitik zu sehen. Dabei steht im Vordergrund, ob ein Konsument in der Geschäftsstätte diese Warengruppe erwartet oder das Unternehmen sogar nur deshalb aufsucht. 46

45 Vgl. Wehrle, F., strategische Marketingplanung in Warenhäusern, a.a.O., S. $195 \mathrm{ff}$.

46 Vgl. Wehrle, F., Strategische Marketingplanung in Warenhäusern, a.a.O., S. 197; Verbundbeziehungen werden von Wehrle explizit nur bei "branching-out"-strategien angeführt, Vgl. ebenda, S. 206. 
Aus Sicht der Verbundanalysen können diese Problemstellungen in zwei Aspekte aufgeteilt werden. Zum einen steht die Frage im Vordergrund, welche kurzfristigen Auswirkungen sich in den aktuellen Kaufsituationen der Kunden ergeben und welche Effekte dies für das Handelsunternehmen hat. Dieser Aspekt wurde bereits im Kontext der Implikationen des Kaufverbundes für den Einzelhandel untersucht. 47 Darüber hinaus sind jedoch die eher langfristig stabilen, am Bedarfsverbund orientierten Beziehungen des Nachfrageverbundes von besonderer Bedeutung.

Wenn das Handelsunternehmen eine Abteilung eliminiert, die sich vor dem Hintergrund der Grundgesamtheit in einer Nachfrageverbundgruppe mit besonders kaufintensiven Abteilungen befindet, so ist z.B. zu erwarten, daß eine Auflösung Auswirkungen auf andere Abteilungen hat. Bei zweifelhaften Fällen bietet sich daher eher eine flächenorientierte Schrumpfung an, um eine vollständige Eliminierung $\mathrm{zu}$ vermeiden. In diesem Zusammenhang ist dann wiederum eine Analyse der Verbundbeziehungen auf Artikelniveau heranzuziehen. So ist das Sortiment innerhalb der betroffenen Abteilung $z u$ analysieren und im Bedarfsfall zu straffen bzw. Sortimentsschwächen sind auszuschalten. 


\section{Schlußbetrachtung}

\section{Zusammenfassende würdigung der Untersuchungsergebnisse}

Aufbauend auf der seit Beginn der 80er Jahre stagnierenden Verbundforschung im Handel wurde in der vorliegenden Untersuchung der versuch unternommen, die verbundanalyse vor dem Hintergrund der komplexen Analysemöglichkeiten, die durch Kundenkarteninformationen eröffnet werden, grundlegend neu aufzuarbeiten. Dabei sollte - entsprechend dem dargelegten zielsystem - zum einen der Verbundbegriff durch die Einbeziehung des zeitraum-Aspektes erweitert werden. Zum anderen waren die bislang vorliegenden Methoden zur Erfassung von Verbundbeziehungen $z u$ analysieren und hinsichtlich der Erfassung des Nachfrageverbundes als zeitraumbezogenes Konstrukt weiterzuentwickeln. In diesem zusammenhang sollte auch überprüft werden, inwieweit die Nachfrageverbundanalyse zur Marktsegmentierung und damit zur Abgrenzung von zielgruppen geeignet ist.

Unter Bezugnahme auf diese zielsetzungen sind die vorliegenden methodischen Ansätze, empirischen Ergebnisse und auch die Implikationen für das Handelsmarketing $z u$ würdigen:

- Unter den methodischen Ansätzen wurde zunächst die clusteranalyse als Verfahren zur Abbildung des Verbundkaufs analysiert. Dabei zeigte sich, daß dieses Verfahren - wie in der überwiegenden Zahl der bislang vorliegenden Forschungsarbeiten zur empirischen Verbundanalyse auch - zur Abbildung des Kaufverbundes als zeitpunktbezogenes Konstrukt geeignet ist. Die vorliegende Datenstruktur auf der Basis von Kundenkarteninformationen ermöglicht allerdings, vor der Verbundanalyse eine clusteranalyse auf der Grundlage soziodemographischer Daten durchzuführen. Dadurch kann der Kaufverbund zielgruppenspezifisch ermittelt werden. 
Zur Analyse des Nachfrageverbundes als zeitraumbezogenes Konstrukt konnte nicht auf bislang in der Verbundforschung vorliegende Analysemethoden zurückgegriffen werden. Als Verfahren zur Aufdeckung von derartigen Verbundbeziehungen kann bei der Datenstruktur von Kundenkarteninformationssystemen die Faktorenanalyse eingesetzt werden. Durch dieses Verfahren gelingt es, Kaufbeziehungen über einen Kaufakt hinaus zu identifizieren und zum Nachfrageverbund zusammenzuführen. Darüber hinaus eröffnet die Faktorenanalyse die Möglichkeit, die Nachfrageverbundstrukturen zur zielgruppenanalyse zu nutzen. Dies schließt sich beim Kaufverbund von vornherein aus.

Als Verfahren zur Zielgruppenbildung kann wiederum auf die clusteranalyse zurückgegriffen werden. Insgesamt eröffnet damit das methodische Vorgehen im Rahmen der Nachfrageverbundanalyse weitere Möglichkeiten zur Erfassung des Kaufverhaltens der Nachfrager und ist vor allem aussagefähiger zur Lösung strategischer Fragen des Handelsmarketing.

- Die empirischen Ergebnisse der vorliegenden Untersuchung haben nachgewiesen, daß das vorgestellte methodische Instrumentarium zur Abbildung empirisch erfaßter Verbundstrukturen geeignet ist. Insbesondere konnten hinsichtlich des Kaufverbundes wesentliche Verbundbeziehungen aufgezeigt werden. Dabei zeigte sich allerdings auch, daß die struktur der Kaufverbundbeziehungen in besonderem Maße durch den Aufbau der Geschäftsstätte geprägt wird. Deshalb ist davon auszugehen, daß der Kaufverbund wesentlich durch situative Einflüsse und dabei nicht zuletzt durch die Plazierung der Produkte beeinflußt wird.

Die Clusteranalyse zur Ermittlung soziodemographischer zielgruppen und eine darauf aufbauende Kaufverbundanalyse 
konnte auf der Basis des vorliegenden Datensatzes jedoch nicht durchgeführt werden. Dies war nicht auf die struktur der Daten, sondern allein auf die zu geringe Anzahl von Verbundkäufen innerhalb der Untergruppen zurückzuführen. Dies zeigt eine spezifische problematik von zielgruppenbezogenen Kaufverbundanalysen auf der Basis von Kundenkarteninformationen auf. Denn trotz des umfangreichen Datensatzes konnte innerhalb der zielgruppen keine ausreichende zellenbesetzung in der Häufigkeitsmatrix erreicht werden. Mit zunehmender Verbreitung von Kundenkarten erscheint aber auch diese Herausforderung lösbar.

Weiterhin zeigt sich im Rahmen der empirischen Analyse des Nachfrageverbundes die Tragfähigkeit des methodischen Instrumentariums. Die faktorenanalytische Verdichtung der Nachfrageverbundstrukturen führt zur Ableitung plausibler Bedarfsmuster der Kundenkartenbesitzer. Diese Bedarfsmuster konnten nicht nur in der Gesamtstichprobe nachgewiesen werden, sondern sind auch innerhalb sozioökonomischer Untergruppen stabil. Dies verdeutlicht nicht zuletzt, daß der Nachfrageverbund im Vergleich zum Kaufverbund erheblich unabhängiger von situativen Einflüssen ist und sich damit dem Bedarfsverbund der Nachfrager annähert. Damit ist der Nachfrageverbund in besonderer Weise aussagefähig für Fragestellungen des strategischen Handelsmarketing.

Anhand der Nachfrageverbundanalyse innerhalb der sozioökonomischen Untergruppen konnten - neben der Identität der Verbundstrukturen - unterschiedliche Kaufintensitäten in den Verbundgruppen nachgewiesen werden. Diese Kaufintensitäten sind vor dem Hintergrund der jeweiligen sozioökonomischen strukturen in den Untergruppen und den damit verbundenen Bedarfsstrukturen plausibel. Dies belegt nicht zuletzt die prognostische Relevanz des vorgestellten Instrumentariums. 
Auf der Grundlage der faktorenanalytisch ermittelten Nachfrageverbundstrukturen lieferte die clusteranalyse hinsichtlich des Nachfrageverbundes homogene Kundensegmente. Diese segmente spiegelten unterschiedliche Nachfragertypen des betrachteten Handelsunternehmens wider. Allerdings zeigte sich auch, daß der typische Warenhauskunde wenig ausgeprägte Raufverhaltensmuster aufweist. Dadurch wird auch die warenhausimmanente zielgruppenproblematik - nämlich die, keine spezifischen zielgruppen gezielt anzusprechen - verdeutlicht.

- Die aufgezeigten methodischen Ansätze, aber auch die empirisch nachgewiesenen Verbundstrukturen lassen wesentliche Implikationen für das Handelsmarketing $z u$. Insbesondere zeigt sich, daß das methodische Instrumentarium in der Handelspraxis zur Verbundanalyse eingesetzt werden kann. Dabei ist vor allem zu betonen, daß in der vorliegenden Untersuchung zwar nur die Verbundbeziehungen zwischen Abteilungen abgebildet wurden, das grundlegende Methodengerüst aber auf jedwede Aggregationsebene, also bspw. auch auf Artikel oder Artikelgruppen übertragen werden kann.

Weiterhin verdeutlichen die aufgezeigten Ergebnisse, daß die Verbundanalyse auf der Grundlage von Kundenkarteninformationen für unterschiedliche Fragestellungen des Handelsmarketing Hilfestellung leisten kann. Dabei ist die Kaufverbundanalyse vor allem für situativ geprägte und damit operative Entscheidungsprobleme wie bspw. Plazierungen oder Sonderangebote einzusetzen. Im Gegensatz dazu liefert die Nachfrageverbundanalyse wesentliche Informationsgrundlagen für langfristig ausgerichtete Entscheidungen wie die sortimentspolitik.

Zusammenfassend ist festzustellen, daß Kundenkarteninformationen aufgrund ihrer objektivität und des Datenumfangs eine 
deutliche Aufwertung der Verbundanalyse im Handel bewirken. Dies gilt um so mehr, als das vorgestellte methodische Instrumentarium für die Abbildung der Verbundstrukturen geeignet ist. Gleichzeitig stellt die Verbundmessung durch ihre vielseitige Anwendbarkeit ein wertvolles Informationsund Entscheidungsunterstützungsinstrument dar.

\section{Ansatzpunkte für die weitere Forschung}

Die methodenorientierte Vorgehensweise und die damit verbundene geschäftsstättenspezifische und abteilungsbezogene empirische Prüfung der Methoden auf ihre Plausibilität führen dazu, daß weitergehende Fragen der Kauf- und Nachfrageverbundforschung offenbleiben mußten. So sind die Implikationen für das Handelsmarketing durch eine weiterführende Replizierung mit Daten anderer Geschäftsstätten und Vertriebsformen zu validieren. Darüber hinaus müssen die zwangsläufig globalen Aussagen zum Kauf- und Nachfrageverbund auf der Basis von Abteilungen durch Untersuchungen auf dem Niveau von Artikeln verfeinert werden. Dazu tritt das Problem, daß der dieser Untersuchung zugrunde liegende Vollsortimenter über keine ausgeprägte zielgruppenausrichtung verfügt und somit die Aussagen für zielgruppenorientierte Handelsunternehmen überprüft werden müssen. Dies ist jedoch aufgrund der allgemeingültigen Ausrichtung des Instrumentariums unproblematisch.

Vor diesem Hintergrund ergeben sich insbesondere die folgenden noch unbearbeiteten Forschungsfelder im Bereich des Kauf- und Nachfrageverbundes:

- Zusammenhänge zwischen dem Aufbau einer Geschäftsstätte und dem in ihr stattfindenden Kaufverbund sind empirisch herzuleiten. Dies ist von besonderer Bedeutung, da eine eindeutige Abhängigkeit von Plazierung und Verbund dazu 
zwingt, nicht-plazierungsbedingte Kaufverbunde als Grundlage für eine Plazierungsplanung heranzuziehen, die dann den plazierungsbedingten Kaufverbund determiniert. Hier stellt sich die Herausforderung, die plazierungsbedingten von den nicht-plazierungsbedingten Kaufverbunden zu trennen.

- Die Erforschung der Kaufverbundbeziehungen innerhalb bestimmter soziodemographischer zielgruppen war aufgrund des zu geringen Datenumfangs in der vorliegenden Untersuchung nicht möglich. Sie bleibt der weiteren Forschung vorbehalten. Daraus können sich umfangreiche neue Erkenntnisse ableiten, wie es in dieser Untersuchung im Rahmen der Nachfrageverbundanalyse möglich war.

- Die Untersuchung von Kauf- und Nachfrageverbunden in zeitreihen ist eine weitere Herausforderung an die wissenschaftliche Erfassung des Verbundes. Da Kunden auch im Zeitablauf zu identifizieren sind, ist es möglich, analog zu Kohortenanalysen feststehende Gruppen im Zeitablauf zu verfolgen und ihre Verbundkäufe $z u$ analysieren. Ergebnisse dieser Verbund-Zeitreihenanalysen besitzen hohe prognostische Relevanz für andere Gruppen, deren Strukturveränderungen im Kaufverbundverhalten ex ante festgestellt werden können.

- Eine Erforschung der Verbundbeziehungen auf Umsatzebene ist bisher im Rahmen der Forschung nicht erfolgt. Dabei stellt vor allem das unterschiedliche Preisniveau verschiedener Abteilungen hohe Anforderungen an eine Handhabung im Bereich der empirischen Forschung. Dennoch können vor allem für die Ableitung wirtschaftlicher wirkungen von Kauf- und Nachfrageverbunden Erkenntnisse von hoher Praxisrelevanz abgeleitet werden. 
- Ein weiteres, hochkomplexes Forschungsfeld stellt die Untersuchung von asymmetrischen verbundbeziehungen dar. Dabei muß entweder hypothesengestützt oder über Befragungen ein komplexes Netz kausaler Beziehungen innerhalb von Verbundkäufen aufgebaut werden. Dazu sind Verbundkaufauslöser und Verbundfolgekauf $z u$ identifizieren. Dabei stellt der Aufbau eines geeigneten Instrumentariums zur empirischen Umsetzung dieser Kausalbeziehungen in ein System von Kauf- und Nachfrageverbunden eine weitere Herausforderung dar. Können diese probleme handhabbar gemacht werden, so besitzen die dann ermittelten Ergebnisse eine hohe informatorische Relevanz für die Einzelhandelsunternehmen. Vor allem die Plazierungs- und Sortimentspolitik kann von solchen Erkenntnissen profitieren.

- Wenn das gesamte system von Kauf- und Nachfrageverbundgrößen von der Forschung vollständig erfaßt ist, so bietet sich daran anschließend eine Kombination von wirkungs- und Verbundforschung an. In diesem zusammenhang muß dann die Möglichkeit untersucht werden, gezielt Verbundbeziehungen aufzubauen und $z u$ beeinflussen. Dies dürfte vor allem im Bereich des Nachfrageverbundes problematisch sein, da dieser sehr stark vom Bedarf geprägt ist. Dennoch bietet eine solche Forschung sowohl der wirkungs- als auch der Verbundforschung große Erkenntnispotentiale.

- Schließlich erscheint es notwendig, das aufgezeigte Beziehungsgefüge zwischen angebots-, wettbewerbs- und konsumentenbezogenen Einflußgrößen und dem Kauf- und Nachfrageverbund umfassend empirisch aufzuarbeiten. Hierzu ist insbesondere erforderlich, die in der vorliegenden Untersuchung dargelegten Beziehungen zu spezifizieren und auf disaggregiertem Niveau zu präzisieren. Darüber hinaus wird eine wesentliche Herausforderung darin bestehen, 
eine derartig umfassende Datenbasis aufzubauen, um die jeweiligen Hypothesen zu prüfen.

Insgesamt wird die Verbundforschung für den Handel dann stetig an Bedeutung gewinnen, wenn es gelingt, einerseits die unterschiedlichen Forschungsschwerpunkte umfassend aufzuarbeiten. Andererseits muß es der Forschung aber auch gelingen, pragmatische Methoden zu entwickeln und plausible Ergebnisse zu erzielen, damit das Handelsmarketing zur Adoption der Methoden und Erkenntnisse bereit ist. Grundsätzlich liegt für den Handel ein hohes Informationspotential in der Aufbereitung von Kaufverhaltensdaten im Rahmen der Verbundforschung. 


\section{Literaturverzeichnis}

Ahlert, D.

Ahlert, D., Günther, $H$.

Ahlert, D., Schröder, $\mathrm{H}$.

Ahlert, D., Schröder, $\mathrm{H}$.

Albach, A.

Aspinwall, L.

Auernhammer, $\mathrm{H}$.

Backhaus, K. , Erichson, B., Plinke, W., Schuchard-Ficher, C., weiber, R. ,
Evolutionäres Handelsmanagement Die controllinggesetzte Evolution erlebnisorientierter Betreibungskonzepte des stationären Einzelhandels, Arbeitspapier Nr. 11 des Lehrstuhls für Betriebswirtschaftslehre, insbesondere Distribution und Handel der Universität Münster, Münster 1990

Strategisches Controlling und experimentelle optimierung im Handel, Arbeitspapier Nr. 8 des Lehrstuhls für Betriebswirtschaftslehre, insbesondere Distribution und Handel der Westfälischen Wilhelms-Universität, 3. Aufl., Münster 1992

Rechtliche Grundlagen des Marketing, stuttgart 1989

"Erlebnisorientierung" im stationären Einzelhandel, in: Marketing ZFP, Heft 4/1990, S. 221-229

Zur Sortimentskalkulation im Einzelhandel, in: Handelsbetrieb und Marktordnung, Festschrift für Carl Ruberg, Wiesbaden 1962, S. 13-40

The characteristics of Goods Theory, in: Managerial Marketing, Lazer, W., Kelley, J. (Hrsg.), Homewood Ill. 1962, S. 633-643

Kommentar zum Bundesdatenschutzgesetz, München 1977

Multivariate Analysemethoden, 4. Aufl., Berlin Heidelberg New York London Paris Tokyo 1987 
Bartels, G.

Bass, F.M., Pessemier, E.A., Tigert, D.J.

Baumbach/Hefermehl

Bierbaum, M.

Bishop, Y.M.M., Fienberg, S.E., Holland, P.W.

Böcker, F.

Böcker, F.

Böcker, F.

Böcker, F.

Böcker, F. ,
Diversifizierung - die gezielte Ausweitung des Leistungsprogramms der Unternehmung, Stuttgart 1966

Complementary and substitute patterns of purchasing and use, in: JAR, 2/1969, S. 19-27

Wettbewerbsrecht, Kommentar, 16. Aufl., München 1990

Leistung und Leistungsbedingungen im Einzelhandel, RationalisierungsGemeinschaft des Handels beim RKW e.V. Köln (Hrsg.), Köln 1979

Discrete Multivariate Analysis Theory and Practice, Boston 1975

Handelskonzentration bei zunehmender Entscheidungskonzentration, in: ma, 6/1986, S. 284-288

Handelskonzentration: Ein partielles Phänomen? - oder: irreführende Handelsstatistiken, in: ZfB, 565. Jg., Nr. 7/1986, S. 654-660

Die Bestimmung der Kaufverbundenheit von Produkten, in: Schriften zum Marketing; Dichtl, E., Böcker, F. (Hrsg.), Bd. 7, Berlin 1978

Die Analyse des Sortimentsverbundes - Eine empirische Untersuchung, in: Perspektiven des Marketing im Handel (Ökonomische Kolloquien 2), Blümle, E.B., Ulrich, W. (Hrsg.), Freiburg 1975, S. 55-81

Mantel "kauft" Bluse - Analyse des 
Merkle, E.

Böcker, F.

Böcker, F. , Merkle, E.

Böcker, F., Kaiser, A., Merkle, E.

Böhme, K.

Borg, I.

Bost, $E$.

Brauer, K.M.

Brede, $\mathrm{H}$.
Sortimentsverbundes, in: Rationeller Handel, 10. Jg., Heft 1 1975, S. $14-20$

Die Analyse des Kaufverbundes - Ein Ansatz zur bedarfsorientierten Warentypologie, in: $\mathrm{ZfbF}, 27$. Jg., Heft 5 1975, S. 290-306

Die Analyse des Sortimentsverbundes, in : Erfolgskontrolle im Marketing, Festschrift für R. Nieschlag, Böcker, F., Dichtl, E. (Hrsg.), Berlin 1975, S. 179-191

Praxistest für multivariate Verfahren, in: asw, Heft $41976, \mathrm{~S}$. $32-43$

Geldkartensysteme auf neuen wegen verpaßte Gelegenheiten oder neue Chancen?, in: bank und markt, $3 / 1988$, S. 5-9

Anwendungsorientierte Multidimensionale Skalierung, Berlin, Heidelberg, New York 1981

Die wirkung der Ladenatmosphäre am Einkaufsort, in: LZ, Nr. 43, Okt. 1986, S. F22-25

Die Ermittlung der optimalen Warengruppenstruktur einer Filiale in Lebensmittelfilialbetrieben, in: Unternehmensforschung im Handel, Gümbel, R., Brauer, K.M., Liebmann, H.P., Müller-Hagedorn, L. (Hrsg.), Rüschlikon-Zürich 1969, S. 53-124

Möglichkeiten zur Bestimmung der Nachfrageverwandschaft mit Hilfe von Indikatoren, Habilitationsschrift, Frankfurt 1974 
Brede, $\mathrm{H}$.

Dahlhoff, H. - D.

Deimel, $\mathrm{K}$.

Dicht1, E., Schobert, R.

Dietrich, M.

Diller, H.

Diller, $\mathrm{H}$.

Ebert, $\mathbf{K}$.

Eckhardt, $K$.
Absatzpolitik mit Hilfe der Nachfrageverwandtschaft, Wiesbaden 1976

Wertorientierungen von Verbrauchern, in: Handwörterbuch der angewandten Psychologie, Bd. 3: Markt und Umwelt, Landsberg 1981, S. 130

Grundlagen des Involvement und Anwendung im Marketing, in: Marketing ZFP, Heft 3, 1989, S. 153-161

Mehrdimensionale Skalierung - Methodische Grundlagen und betriebswirtschaftliche Anwendungen, München 1979

Konsument und Gewohnheit - Eine theoretische und empirische Untersuchung zum habituellen Käuferverhalten, Heidelberg 1986

Die Wirkung von Verkäufen unter Einstandspreisen im Lebensmitteleinzelhandel, in: DBW, 41. Jg., 1981 , S. 409-418

Preispolitik, 2. Aufl., stuttgart, Berlin, Köln 1991

Warenwirtschaftssysteme und Warenwirtschaftscontrolling, Schriften $\mathrm{zu}$ Distribution und Handel, Bd. 1, Ahlert, D. (Hrsg.), Frankfurt a.M. 1986

Die Sonderangebotspolitik in Warenhandelsbetrieben - eine empirische Studie, Wiesbaden 1976 
Eggert, U.

Ehrenberg, A.S.C.

Engelhardt, W.H.

Fischer, T.

Fry, J.N.

Gerken, G.

Gemünden, H.G.

Guikley, D.K., Schmidt, P.

Hamerle, A., Tutz, G.
Bargeldlose Zahlungsmittel im Einzelhandel, in: Der Sport-Markt, $1 / 1989$, S. 54-56

Repeat buying, Amsterdam 1972

Erscheinungsformen und absatzpolitische Probleme von Angebots- und Nachfrageverbunden, in: ZfbF 1976, S. 77-90

Die Warenkorbanalyse als Instrument zur experimentelien Verbesserung des Einsatzes absatzseitiger Aktionsparameter im Lebensmitteleinzelhandel - Theorie, Methodik und empirische Analyse, Diskussionspapier des Lehrstuhls für Betriebswirtschaftslehre, insbesondere Distribution und Handel der Universität Münster, Münster 1991

Family branding and consumer brand choice, in: JMR 1967, S. 237-247

Die Zukunft des Handels, in: MJ, $4 / 1987$, S. 300-302

Wahrgenommenes Risiko
Informationsnachfrage
systematische Bestandsaufnahme der
empirischen Befunde, in: Marketing
ZFP, Heft 1, Febr. 1985, S. 27-32

Some Small Sample Properties of Estimators and Test statistics in the Multivariate Logit Model, in: Journal of Econometrics, 10. Jg., 1979, S. 33-42

Zusammenhangsanalysen in mehrdimensionalen Kontingenztabellen, in: Fahrmeier, L., Hamerle, A. (Hrsg.), Multivariate statistische Verfahren, Wien 1983, S. 473-574 
Hansen, U.

Hauzeneder, R.

Heemeyer, $\mathrm{H}$.

Heidel, B.

Heinemann, G.

Heinemann, G.

Heinemann, M.

Heinen, $\mathbf{E}$.

Heiner, V.
Absatz- und Beschaffungsmarketing des Einzelhandels, Teil 2, Göttingen 1976

Der Sortimentsverbund im Einzelhandel, Diss., München 1975

Psychologische Marktforschung im Einzelhandel - Entwicklung und Test einer operationalen Befragungs- und Auswertungskonzeption, Wiesbaden 1981

Die Nutzung von Scannerdaten zur Planung der Absatzpolitik in Einzelhandelsbetrieben, unveröffentl. Manuskript, Diss. Trier 1989

Betriebstypenprofilierung im Facheinzelhandel - Ansätze der strategischen Marktsegmentierung, Arbeitspapier Nr. 35 des Instituts für Marketing der Universität Münster, Meffert, H. (Hrsg.), Münster 1987

Betriebstypenprofilierung und Erlebnishandel - Eine empirische Analyse am Beispiel des textilen Facheinzelhandels, Wiesbaden 1989

Einkaufsstättenwahl und Firmentreue des Konsumenten, Münster 1974

Grundlagen betriebswirtschaftlicher Entscheidungen - Das Zielsystem der Unternehmung, 3. Aufl., in: Die Betriebswirtschaft in Forschung und Praxis, Heinen, E., Börner, D., Kappler, E., Kirsch, w., Meffert, H., (Hrsg.), Bd.1, Wiesbaden 1976

Tankstellen Vorreiter für Handel ?, In: Dynamik im Handel, 12/1990, S. $12-16$ 
Hermann, T.

Horacek, M.

Hruschka, H.

Hruschka, H.

Hüttermann, M.U.

Hüttner, M.

Humbel, P.

Huwyler, M.

Kaas, R.,

Dietrich, M.

Kerlinger, F.N.
Co-branding: Sieche Patienten, in: à la card Aktuell, Nr. 12/1991, S. 15

Der kalkulatorische Ausgleich, Wien 1950

Folgegeschäfte - Zur Bestimmung der Umsatzverbundenheit von Haupt- und Zusatzprodukten mit $\mathrm{Hilfe}$ der Polynominal-Lag-Regression, in: Marketing ZFP 1983, S. 165-169

Bestimmung der Kaufverbundenheit mit Hilfe eines probabilistischen Meßmodells, in: $\mathrm{ZfbF} 43,5 / 1991, \mathrm{~s}$. 418-434

Formen und Risiken des co-branding, Hamburg 1991

Die Kohortenanalyse als Instrument der strategischen Marktforschung, in: Realisierung des Marketing, Belz, C. (Hrsg.), Bd. 1, St. Gallen 1986 , S. 309-327

Preispolitische Gewinndifferenzierung im Einzelhandel, Zürich 1958

Sortiment und Kaufhandlung, Diss., Bern Zürich 1966

Die Entstehung von Kaufgewohnheiten bei Konsumgütern, in: Marketing, Heft 1, 1979, S. 13-21

Grundlagen der Sozialwissenschaften, Bd. 1, deutsche Übersetzung, Weinheim-Basel 1979 
Klein, s.

Knoblich, H.

Knoblich, H.

Koch, H.

Köhler, R.

Kotler, $\mathrm{Ph}$.

Krelle, w.

Kreuzer, R.T. Weppler, K. ,

Kroeber-Riel, W.

Kroeber-Riel, W.
Electronic cash-System: Langer Weg mit Hindernissen, in: à la card $\mathrm{Ak}^{-}$ tuell, Nr. 23, 1990, S. 6-11

Betriebswirtschaftliche Warentypologie - Grundlagen und Anwendungen, Köln Opladen 1969

Warentypologie, in: Handwörterbuch der Absatzwirtschaft, Tietz, B. (Hrsg.), Stuttgart 1974, Sp. 21752184

Die große Lust am Kartenspiel, in: Manager Magazin, 11/1989, S. 276287

Grundprobleme der strategischen Marketingplanung, in: Die Führung des Betriebs, Geist, M., Köhler, R. (Hrsg.), Stuttgart 1981, S. 261-291

Marketing decision making: A model building approach, New York 1971

Präferenz- und Entscheidungstheorie, Tübingen 1968

Marketing by Database, Eine Herausforderung für Markenartikler, in: Markenartikel 10/1990, S. 488499

Informationsüberlastung durch Massenmedien und Werbung in Deutschland, Messung - Interpretation Folgen, in: DBW, Heft 31987 , S. 257-264

Zentrale Probleme auf gesättigten Märkten, in: Marketing ZFP, Heft 3, 1984, S. 210-214 


\begin{abstract}
Kroeber-Riel, W.
Konsumentenverhalten, München 1990

4. Aufl.,

Kupsch, P.

Unternehmensziele, stuttgart, New York 1979

Kupsch, P., Hufschmied, P.

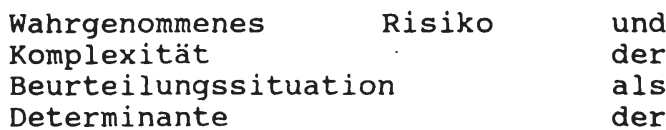
Qualitätsbeurteilung, in: Meffert, H. ' Steffenhagen, H. , Freter, H. Information, Wiesbaden 1979, S. $225-278$
\end{abstract}

Liedtke, P.

Absatz- und Kostenverbundenheiten handelsbetrieblicher Leistungskomponenten und Leistungen und ihre Bedeutung für die Sortimentspolitik der Handelsbetriebe, Diss., Gießen 1969

Lienert, G.A.

Verteilungsfreie Methoden der Biostatistik, Bd. 1, 2. Aufl., Meisenheim 1973

Lipson, H.A., Darling, J.R., A Two-Phase Interaction Process for Marketing Model Construction, in: Reynolds, F.D. MSU Business Topics, Autumn 1970, S. $34-44$

Medla, K. Shop-in-the-Shop, ein Konzept der Angebotspräsentation, München 1987

Meffert, $H$.

Marketingstrategien der Warenhäuser - Wege aus der Krise?, in: Harvard Manager, Heft 2, 1985, S. 20-28

Meffert, $\mathrm{H}$.

Wettbewerbsorientierte Marketingstrategien im Zeichen schrumpfender und stagnierender Märkte, in: strategisches Marketing, Raffée, H., Wiedmann, K.-P. (Hrsg.), stuttgart 1989, S, 475-490 
Meffert, $\mathrm{H}$.

Meffert, $H$.

Meffert, $H$.

Meffert, $H$.

Meffert, $\mathrm{H}$.

Meffert, H., Steffenhagen, $\mathrm{H}$.

Meffert, H., Walters, M.
Der neue Konsument: Chance für das Marketing, in: IHA-News, Heft 2, 1986

Marketingstrategien in stagnierenden und schrumpfenden Märkten: $\mathrm{Be}-$ triebswirtschaftliche Entscheidungen bei stagnation, Edmund Heinen zum 65. Geburtstag, Pack, L., Börner, D. (Hrsg.), Wiesbaden 1984, S. $37-72$

Strategische Planungskonzepte in stagnierenden und gesättigten Märkten, in: DBW 1983, S. 193-209

Marktorientierte Führung in stagnierenden und gesättigten Märkten, Arbeitspapier Nr. 9 der Wissenschaftlichen Gesellschaft für Marketing und Unternehmensführung e.V., Meffert, H., Wagner, H. (Hrsg.), Münster 1983

Marketingforschung und Käuferverhalten, Wiesbaden 1992, S. 257 ff.

Marketing-Prognosemodelle, Quantitative Grundlagen des Marketing, stuttgart 1977

Anpassung des absatzpolitischen Instrumentariums in stagnierenden und schrumpfenden Märkten, in: Betriebswirtschaftliche Beiträge zur Bewältigung der ökonomischen Krise, Staehle, W.M., stoll, E. (Hrsg.), Wiesbaden 1984, S. 141-160

Marketing - Grundlagen der Absatzpolitik, 7. Auf1., Wiesbaden 1986

Marktforschung, Wiesbaden 1986 
Meffert, $\mathrm{H}$.

Merkle, E.

Michalek, M.

Monhemius, K.Ch.

Morgen, R.D.v.

Mohme, J .

Müller-Hagedorn, L.

Müller-Hagedorn, L. Heidel, B.
Erfolgsfaktoren im Einzelhandelsmarketing, in: Marketing-Erfolgsfaktoren im Handel, Bruhn, M., (Hrsg.), Frankfurt/Main New York 1987 , S. $13-45$

Die Erfassung und Nutzung von Informationen über den Sortimentsverbund in Handelsbetrieben, in: Schriften zum Marketing, Dichtl, E., Böcker, F. (Hrsg.), Bd. 11, Berlin 1981

Co-branding: Chance oder Bedrohung, in: à la card Aktuell, Nr. 12/1991, S. 11-14

Umweltbewußtes Kaufverhalten von Konsumenten, Bd. 18 der Schriften $\mathrm{zu}$ Marketing und Management, Meffert, H. (Hrsg.), Frankfurt a.M. 1992

Neues Gesetz: Die Kreditkarte als Verbraucherkredit, in: à la card Aktuell, Nr. 22, 1990, S. 6-9

Der Einsatz von Kundenkarten im Einzelhandel - Konzeptionelle und praktische probleme kartengestützter Kommunikationssysteme und Kundenbindungsstrategien im stationären Einzelhandel, Diss. (unveröffentlichtes Manuskript), Münster 1991

Das Problem des Nachfrageverbundes in erweiterter sicht, in: $2 \mathrm{fbF}$ 1978, S. 181-193

Plazierungspolitik nach dem Verbundkonzept im stationären Einzelhandel -Eine Wirkungsanalyse, in: Marketing ZFP, 1/1989, S. 19-26 
Nevans, $R$.

Nieschlag, R., Dichtl, E. Hörschgen, H.

Nieschlag, R., Dichtl, E., Hörschgen, $\mathrm{R}$.

Ost, F.

ott, A.E.

o.V.

o.v.

o.V.

o.v.

o.v.
Stores slow to follow Sears'Lead as major financial Services supplier, in: Retail Banker, $2 / 51988$, S. 1012

Marketing - Ein entscheidungsorientierter Ansatz, 7. Auf1., Berlin 1974

Marketing, 13. Aufl., Berlin 1983

Faktorenanalyse, in: Multivariate statistische Verfahren, Fahrmeir, L., Hamerle, A. (Hrsg.), Berlin etc. 1984 , S. 575-662

Grundzüge der Preistheorie, Göttingen 1968

Komplettabddruck des Urteils der dritten Kammer für Handelssachen am Landgericht Mannheim gegen die Goldene Kundenkarte von Hertie, in: à la card Aktuell, Nr.3 Extra, 1989, S. $1-8$

Handelskarten: Orden für stammkunden, in: à la card Aktuell, Nr. 11, 1991, S. 36-40

Co-branding: Die Luft ist raus, in: à la card Aktuel1, Nr. 12, 1991, S. 6-10

Hertie und Barclays bauen optimus Bank aus, in: FAZ vom 15.8.1991, $S$. 12

Mittel zur engeren Kundenbindung, in: Handelsblatt 9/8/1989, S. 11 
o.v.

o.v.

o.v.

o.v.

o.v.

o.v.

o.v.

o.v.

o.v.

o.v.

o.v.

o.v.

o.v.

Mit der Vertrauenskarte Kunden binden, in: Textilwirtschaftschaft, $\mathrm{Nr} .49,12 / 1989$, S. 12

Kundenkarten im Einzelhandel, BTE Fachdokumentation, Köln 1990

Die Karten werden neu gemischt, in: test $2 / 90$, S. $15-21$

à la card, 2. Aufl., Hamburg 1989

Was Kundenkarten bieten, in: DM $2 / 91$, S. 63

Zucker für Treulose, in: DM 2/91, S. $60-64$

Im Dialog mit dem Kunden, in: Textilwirtschaft, 9/1989, S. 26

Die Basis für den Dialog, in: asw, 4/1990, S. 104-113

Handel 2000, in: Sonderdruck der LZ, Nr. 52, 20.12.1982, S. 1-14

Statistisches Jahrbuch der Bundesrepublik Deutschland, 0.0., 1991

Hertie gewinnt den Prozeß, in: Textilwirtschaft, Nr. 49, 12/1989, s. 23

Das Raster wird feiner, in: Textilwirtschaft, $9 / 1989$, S. 32

Königsweg zum Kunden?, in: asw $2 / 91$, S. $55-57$ 
o.V.

o.V.

o.V.

o.V.

o.V.

o.V.

Patt, P. -J.

Poggenpohl, M.

Poynor Burns, R.

Raffée, H. , Wiedmann, K.P.
ec-Karte und Geheimzahl: Genügt das...?, in: ehb $10 / 90$, S. 766-768

Dezentral macht stark, in: $w \& v, N r$. $44 / 2$, Nov. 1990 , S. 14

Ihr Grundstein für eine treffsichere Direktwerbung, in: ehb 1/91, S. $42-45$

Nielsen Report 1988, Frankfurt 1989

Nielsen Report 1989, Frankfurt 1990

BAG-Untersuchung 1988, Köln 1989

Strategische Erfolgsfaktoren im Einzelhandel, Schriften zu Marketing und Management, Bd. 14, Meffert, H. (Hrsg.), Frankfurt a.M., Bern, New York, Paris 1988

Kundenkarteninformationen als Instrument der Verbundanalyse im Einzelhandel, Arbeitspapier Nr. 40 des Instituts für Marketing der Universität Münster, Meffert, H. (Hrsg.), Münster 1991

Discover Card: An industry surprise, in: Cards international, $29 / 1 / 1990$, S. 13-15

Wertewandel und gesellschaftsorientiertes Marketing - Die Bewährungsprobe strategischer Unternehmensführung, in: Strategisches Marketing, Raffée, H., Wiedmann, K.P. (Hrsg.), S. 552-611 
Rentz, J.O., Reynolds, F.D., stout, R.G.

Revenstorf, D.

Reynolds, D.F., Rentz, J.O.

Riebel, P.

Robinson, J.

Rühli, E.

Sabel, H.

Schmitz, G.

Schneider, M.

Schnetkamp, G.
Analysing Changing Consumption Patterns with Cohort Analysis, in: Journal of Marketing Research, Vol. 7, Feb. 1983, S. 12-20

Lehrbuch der Faktorenanalyse, stuttgart 1976

Cohort Analysis: An Aid to strategic Planning, in: Journal of Marketing, Vol. 45, summer 1981, S. 62-70

Kosten und Preise bei verbundener Produktion, Substitutionskonkurrenz und verbundener Nachfrage, opladen 1971

The economics of imperfect competition, London, New York 1961

Optimale Sortimentsplanung mit linearer Programmierung, Zürich 1963

Produktpolitik in absatzwirtschaftlicher Sicht - Grundlagen und Entscheidungsmodelle, Wiesbaden 1971

Kalkulatorischer Ausgleich als betriebspolitische Aufgabe, in: Distributionswirtschaft, Festausgabe zum 75. Geburtstag von Prof. Dr. R. Seyffert, Sundhoff, E. (Hrsg.), Köln Opladen 1968, S. 1-27

Aufbruch in eine andere welt, in: manager magazin, 11/1990, S. 316323

Einstellungen und Involvement als Bestimmungsfaktoren des sozialen Verhaltens, Frankfurt a.M. 1982 
Schultz, H.

Schweiger, H.,

Mazanec, J.,

wiegele, 0 .

silberer, G.

Simon, H.

Simon, H.

Simon, H. , Kucher, E.

Simon, H., Kucher, E.,

Sebastian, K. $-\mathrm{H}$.

Sova, 0., Piper, J.

Spannagel, R.
The Theory and Measurement of Demand, 4. Aufl., Chicago 1962

Das Modell des "erlebten Risikos" ("perceived risk"). Struktur und operationalisierungskonzepte, in: Der Markt, 60/1976, S. 94-98

Die Bedeutung und Messung von Kauferlebnissen im Handel, in: Handelsforschung 1989, Jahrbuch der Forschungsstelle für den Handel Berlin (FfH) e.V., Trommsdorff, V. (Hrsg.), Wiesbaden 1989, S. 59-76

Preismanagement, Wiesbaden 1982

Entscheidungsunterstützung mit Scanner-Daten, in: Marketing im technologischen Umbruch, Simon, $H$. (Hrsg.), Stuttgart 1987, S. 63-75

Höhere Marketing-Effizienz mit Scannerdaten: in: asw, $2 / 1983, \mathrm{~S}$. 83-89

Scannerdaten in Marketingforschung und Marketingentscheidung, in: $\mathrm{ZfB}$ 1982 , S. 555-579

Computergestützte Warenwirtschaft im Handel, Köln 1985

Strukturwandel im Einzelhandel durch Fußgängerzonen, in: FfH Mitteilungen, Januar 1979 , S. 1-6 
Stahl, P.

Steffenhagen, $\mathrm{H}$.

Steffenhagen, $\mathrm{H}$.

Steffenhagen, H., Heemeyer, $\mathrm{H}$. Grannemann, R.

Steinhausen, D. , Langer, $\mathrm{K}$.

stock, E., Wichert, R.

Tinnefeld, Tubies

Trommsdorff, V.

Verbundwirkungen im Sortiment - Ein Beitrag zur Erfassung und Messung von Verbundwirkungen im Sortiment von Handelsbetrieben, Diss. Münster 1977

Kommunikationswirkung - Konsequenzen der Kommunikationsarbeit, Schriften zur Kommunikationsarbeit, Dahlhoff, H.-D. (Hrsg.), Bonn 1983

Entscheidungsverhalten unter zeitdruck, in: DBW, 4/1978, S. 595598

Bekanntheitsverbund zwischen Warenhausabteilungen - Eine psychographische Verbundanalyse, Arbeitspapier Nr. 81/08 des Instituts für Wirtschaftswissenschaften der RWTH Aachen - Lehrstuhl für Betriebswirtschaftspolitik und Marketing, Aachen 1981

Clusteranalyse, Berlin, New York 1977

Die Führungskraft im Handel als Kulturmanager, in: BddW, Nr. 92 vom 15.5.1990, S. 7

Datenschutzrecht, München 1988

Wertewandel und Wandel im Handel, in: Handesforschung 1986, Jahrbuch der Forschungsstelle für den Handel, Berlin (FfH) e.V., Trommsdorff, V. (Hrsg.), Heidelberg 1986, s. $3-16$

Konsumentenverhalten, 1989

stuttgart

Trommsdorff, $V$.

Faktorenanalyse, Berlin, Heidelberg, New York 1972 
Usslar, L. v.

Usslar, I. V.,

Morgen, R.D. v.

Wehrle, F.

Weiber, R.

Weinberg, G.M.

Weinberg, P.

Weinberg, P.

Weinhold-Stünzi, $H$.

Weller, M.

Werner, J.
Anmerkungen zum Hertie-Urteil: Rechtssicherheit oder nicht, in: à la card Aktuell, Nr. 1, 1990, S. 18-19

Aktuelle Rechtsfragen der Kreditkartenpraxis, Hamburg 1989

Strategische Marketingplanung in Warenhäusern, Schriften zum Marketing, Bd. 1, Meffert, H. (Hrsg.), Frankfurt a.M. 1981

Faktorenanalyse, st. Gallen 1984

Marktforschung im Handel, in: Handbuch der Marktforschung, 2 . Halbbd., Behrens, K.C. (Hrsg.), Wiesbaden 1977 , S. 1229-1239

Das Entscheidungsverhalten der Konsumenten, Paderborn 1981

Erlebnisorientierte Einkaufsstättengestaltung im Einzelhandel, in: Marketing ZFP, Heft 2/1986, S. 97102

Grundzüge des Kundenstamm-Marketing, in: Thexis, Nr. 2, 1987, S. 8-14

Das Kreditkartenverfahren, Köln 1986

Einstellungen zum Produkt und Einstellungen zum Produktbereich als Grundlage einer Konsumententypologie Anwendungsbeispiele für einen ausgewählten produktmarkt, in: Marketing ZFP, Heft 3, Aug. 1982, S. 158 
Windhorst, K.-G.

Zeichen, A.

Zellekens, H.J. Fontaine, J., Braatz, F.,

Zellekens, H.J.

Zentes, J.

Zentes, J.
Wertewandel und Konsumentenverhalten. Ein Beitrag zur empirischen Analyse der Konsumrelevanz individueller Wertvorstellungen in der Bundesrepublik Deutschland, Schriften der Wissenschaftichen Gesellschaft für Marketing und Unternehmensführung e.V., Münster 1985

Die Darstellung der Auftragsgrößenstruktur und die Möglichkeiten ihrer Veränderung im Absatzbereich der Unternehmung, in: Die Unternehmung, 1974 , S. 199-212

Zahlung per Karte, Köln 1989

Kundenkarten - das neue MarketingMedium ?, in: Dynamik im Handel, $12 / 1990$, s. $2-7$.

Tendenzen der Entwicklung von warenwirtschaftssystemen, in: Moderne Warenwirtschaftssysteme im Handel, Berlin 1984

Warenwirtschaftssysteme - Auf dem Weg zum Scientific Management im Handel, in: Marketing ZFP, Heft 3, 1988 , S. $177-181$ 
Marcus Poggenpohl - 978-3-631-75099-5

Downloaded from PubFactory at 01/11/2019 08:22:38AM

via free access 


\section{SCHRIFTEN ZUM MARKETING}

Band 1 Friedrich Wehrle: Strategische Marketingplanung in Warenhäusern. Anwendung der Portfolio-Methode. 1981. 2. Auflage. 1984.

Band 2 Jürgen Althans: Die Übertragbarkeit von Werbekonzeptionen auf internationale Märkte. Analyse und Exploration auf der Grundlage einer Befragung bei europaweit tätigen Werbeagenturen. 1982.

Band 3 Günter Kimmeskamp: Die Rollenbeurteilung von Handelsvertretungen. Eine empirische Untersuchung zur Einschätzung des Dienstleistungsangebotes durch Industrie und Handel. 1982.

Band 4 Manfred Bruhn: Konsumentenzufriedenheit und Beschwerden. Erklärungsansätze und Ergebnisse einer empirischen Untersuchung in ausgewählten Konsumbereichen. 1982.

Band 5 Heribert Meffert (Hrsg.): Kundendienst-Management. Entwicklungsstand und Entscheidungsprobleme der Kundendienstpolitik. 1982.

Band 6 Ralf Becker: Die Beurteilung von Handelsvertretern und Reisenden durch Hersteller und Kunden. Eine empirische Untersuchung zum Vergleich der Funktionen und Leistungen. 1982.

Band 7 Gerd Schnetkamp: Einstellungen und Involvement als Bestimmungsfaktoren des sozialen Verhaltens. Eine empirische Analyse am Beispiel der Organspendebereitschaft in der Bundesrepublik Deutschland. 1982.

Band 8 Stephan Bentz: Kennzahlensysteme zur Erfolgskontrolle des Verkaufs und der MarketingLogistik. Entwicklung und Anwendung in der Konsumgüterindustrie. 1983.

Band 9 Jan Honsel: Das Kaufverhalten im Antiquitätenmarkt. Eine empirische Analyse der Kautmotive, ihrer Bestimmungsfaktoren und Verhaltenswirkungen. 1984.

\section{SCHRIFTEN ZU MARKETING UND MANAGEMENT}

Band 10 Matthias Krups: Marketing innovativer Dienstleistungen am Beispiel elektronischer Wirtschaftsinformationsdienste. 1985.

Band 11 Bernd Faehsler: Emotionale Grundhaltungen als Einflußfaktoren des Käuferverhaltens. Eine empirische Analyse der Beziehungen zwischen emotionalen Grundhaltungen und ausgewählten Konsumstrukturen. 1986.

Band 12 Ernst-Otto Thiesing: Strategische Marketingplanung in filialisierten Universalbanken. Integrierte Filial- und Kundengruppenstrategien auf der Grundlage erfolgsbeeinflussender Schlüsselfaktoren. 1986.

Band 13 Rainer Landwehr: Standardisierung der internationalen Werbeplanung. Eine Untersuchung der Prozeßstandardisierung am Beispiel der Werbebudgetierung im Automobilmarkt. 1988.

Band 14 Paul-Josef Patt: Strategische Erfolgsfaktoren im Einzelhandel. Eine empirische Analyse am Beispiel des Bekleidungsfachhandels. 1988. 2. Auflage. 1990.

Band 15 Elisabeth Tolle: Der Einfluß ablenkender Tätigkeiten auf die Werbewirkung. Bestimmungsfaktoren der Art und Höhe von Ablenkungseffekten bei Rundfunkspots. 1988.

Band 16 Hanns Ostmeier: Ökologieorientierte Produktinnovationen. Eine empirische Analyse unter besonderer Berücksichtigung ihrer Erfolgseinschätzung. 1990.

Band 17 Bernd Büker: Qualitätsbeurteilung investiver Dienstleistungen. Operationalisierungsansätze an einem empirischen Beispiel zentraler EDV-Dienste. 1991.

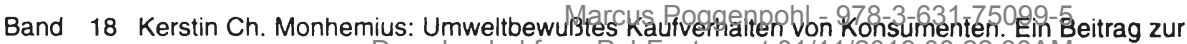

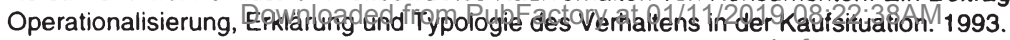


Band 19 Uwe Schürmann: Erfolgsfaktoren der Werbung im Produktlebenszyklus. Ein Beitrag zur Werbewirkungsforschung. 1993.

Band 20 Ralf Birkelbach: Qualitätsmanagement in Dienstleistungscentern. Konzeption und typenspezifische Ausgestaltung unter besonderer Berücksichtigung von Verkehrsflughäfen. 1993.

Band 21 Simone Frömbling. Zielgruppenmarketing im Fremdenverkehr von Regionen. Ein Beitrag zur Marktsegmentierung auf der Grundlage von Werten, Motiven und Einstellungen. 1993.

Band 22 Marcus Poggenpohl: Verbundanalyse im Einzelhandel auf der Grundlage von Kundenkarteninformationen. Eine empirische Untersuchung von Verbundbeziehungen zwischen Abteilungen. 1994. 


\section{Peter Wahle}

\section{Erfolgsdeterminanten im Einzelhandel}

Eine theoriegestützte, empirische Analyse strategischer Erfolgsdeterminanten, unter besonderer Berücksichtigung des Radiound Fernsehfacheinzelhandels

Frankfurt/M., Bern, New York, Paris, 1991. XVI, 328 S., 37 Abb. Marktorientierte Unternehmensführung. Herausgegeben von Hermann Freter. Bd. 12

ISBN 3-631-43533-9 br. DM 97.--

Mit dem berühmten PIMS-Projekt und dem Bestseller von Peters/ Waterman, "Auf der Suche nach Spitzenleistungen", wurde die planerische Auseinandersetzung mit den Determinanten und Ursachen des Unternehmenserfolges die zentrale Aufgabe des strategischen Managements. Für den Einzelhandel gilt das aufgrund der hohen Wettbewerbsintensität und den wenigen wissenschaftlichen Beiträgen zu diesem Thema in besonderem Maße. Vor diesem Hintergrund weist die Arbeit eine Reihe von beachtlichen Vorteilen für die Erklärung von Erfolgsunterschieden im Einzelhandel auf:

1) die neue Differenzierung nach management-, wettbewerbsbranchen- und konsumentenspezifischen Determinanten

2) die branchenübergreifende, branchen- und regionalmarktspezifische Analyse

3) die situative Spezifizierung

4) die Einbeziehung einer großen Zahl an handelsspezifischen Erfolgsindikatoren.

Aus dem Inhalt: Begriff von Erfolgsdeterminanten und -indikatoren Merkmale von Erfolgsdeterminanten - Diskussion bekannter Forschungsansätze - PIMS-Modell, Wettbewerbsstrategieforschung Explorativer und multivariater empirischer Test bei über 130 Unternehmen und 430 Konsumenten 
Marcus Poggenpohl - 978-3-631-75099-5

Downloaded from PubFactory at 01/11/2019 08:22:38AM

via free access 